\title{
Relaxationen in komplexen Fluiden
}

\author{
Dissertation
}

\author{
zur Erlangung des Doktorgrades \\ der Mathematisch-Naturwissenschaftlichen Fakultät \\ der Georg-August-Universität zu Göttingen
}

vorgelegt von

Moritz Schwabe

aus

Preetz

Göttingen, 2010 
Referent: Prof. Dr. Konrad Samwer

Korreferent: Prof. Dr. Hans-Ulrich Krebs

Tag der mündlichen Prüfung: 02. 11. 2010 
Für meine geliebte Ehefrau 



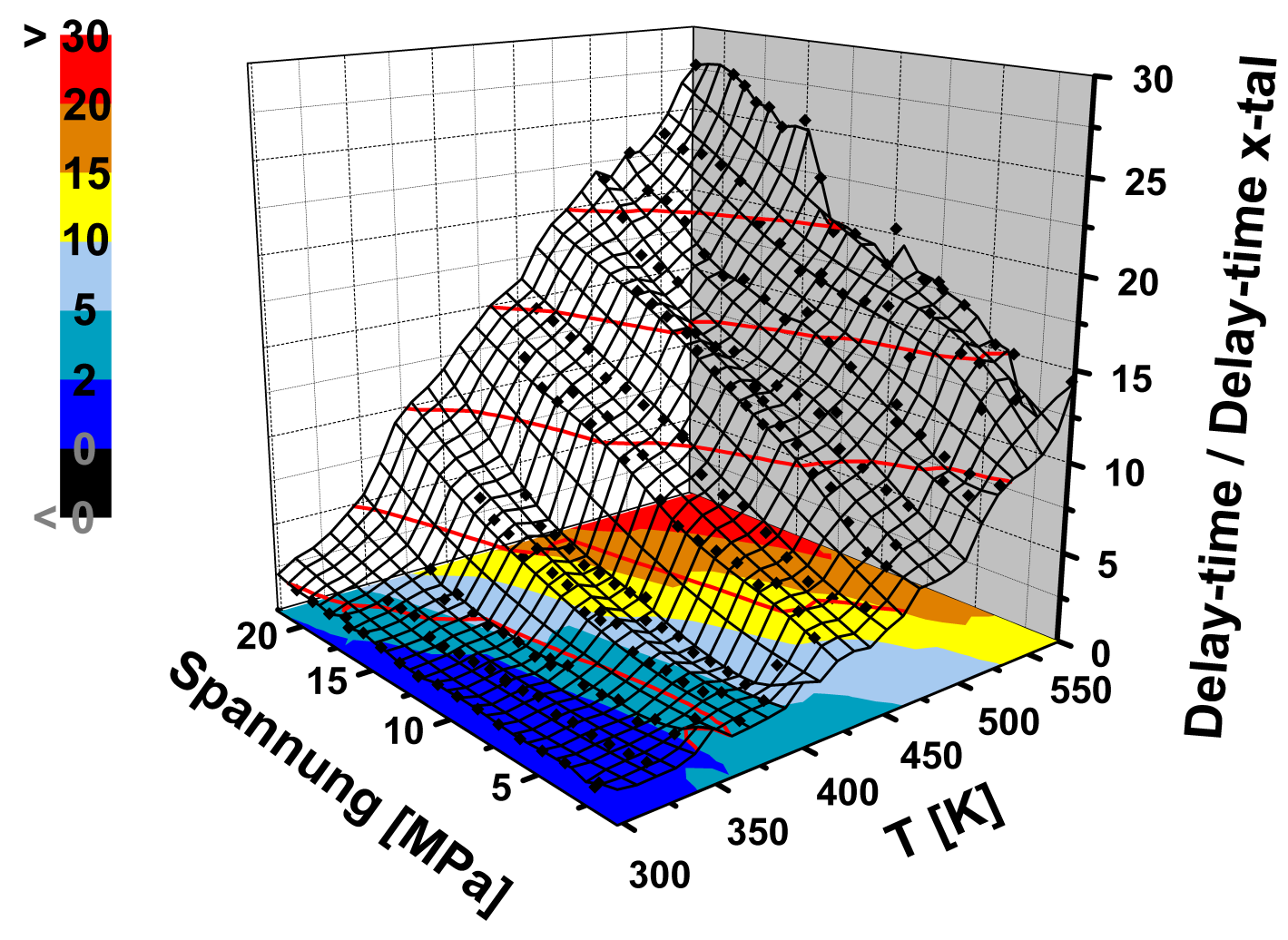

Die Wahrheit ist eben kein Kristall, den man in die Tasche stecken kann, sondern eine unendliche Flüssigkeit, in die man hineinfällt.

(Robert Musil) 



\section{Inhaltsverzeichnis}

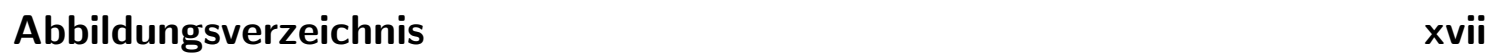

1 Einleitung 1

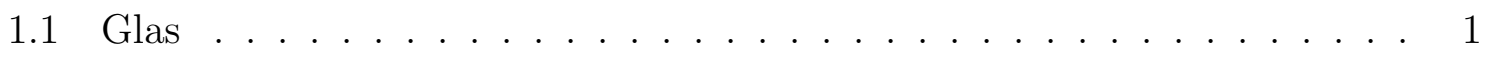

1.1.1 Physik des Glasübergangs . . . . . . . . . . . . . . . . . 2

1.1.2 Unterschied zwischen Gläsern und Flüssigkeiten . . . . . . . . . . 3

1.2 Grundlagen der Viskoelastizität . . . . . . . . . . . . . 5 5

1.3 Dynamisch-mechanische Messungen an viskoelastischen Materialien . . . 7

1.3.1 DMA ......................... 7

1.3.1.1 Messung mit einer dynamischen Kraft . . . . . . . . 7

1.3.1.2 Spannungs-Dehnungskurven . . . . . . . . . . . . . 9

1.3.1.3 Creep/Recoverymessungen . . . . . . . . . . . . 12

1.3.2 Weitere wichtige Spektroskopiearten . . . . . . . . . . . . 13

1.3.2.1 Ultraschalluntersuchungen . . . . . . . . . . . 13

1.3.2.2 Atomic force acoustic microscopy (AFAM) . . . . . . 13

1.3.2.3 Doppelpaddel-Oszillator (DPO) . . . . . . . . . 14

1.4 Relaxationen im Glaszustand . . . . . . . . . . . . . . . 15

1.4.1 Potenzielle Energielandschaft (PEL) . . . . . . . . . . . . . . . 21

1.4.2 Alterungsprozesse . . . . . . . . . . . . . . 22

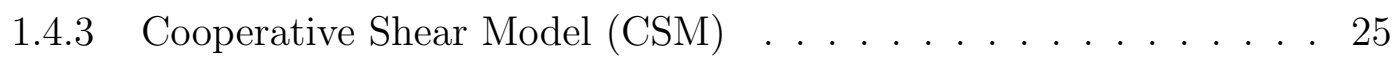

1.4.4 Längenskalen von mikroskopischen Prozessen - Aktivierungsvolumen 27

1.5 Polymerdynamik . . . . . . . . . . . . . . . . . 30

1.5.1 Kurbelwellen- und Seitengruppenbewegungen . . . . . . . . . . 31

1.5.2 Chemical Confinement . . . . . . . . . . . . . . . . . 32 
2 Material und Methoden $\quad 35$

2.1 Metallische Proben . . . . . . . . . . . . . . . . . . . 35

2.1.1 Mechanische Messungen von metallischen Proben . . . . . . . . . 36

2.1.2 Dynamisch-mechanische Analysen . . . . . . . . . . . . . . . 38

2.1.3 Aufnahme von Spannungs-Dehnungskurven . . . . . . . . . . . 38

2.1.4 Analysen von Creep/Recoverykurven . . . . . . . . . . . . . . . 38

2.2 Polymerproben . . . . . . . . . . . . . . . . . . . . 40

2.3 Charakterisierung der Polymerproben . . . . . . . . . . . . . . . 42

2.4 Dynamisch-mechanische Analysen der Polymerproben . . . . . . . . . . . 43

$\begin{array}{lll}3 & \text { Ergebnisse } & 45\end{array}$

3.1 Relaxationen in amorphen Metallen . . . . . . . . . . . . . . . . . . 45

3.1.1 Heizratenabhängige Relaxationen . . . . . . . . . . . . . . . . . . 45

3.1.2 Untersuchung von Spannungs-Dehnungskurven . . . . . . . . . . 55

3.1.3 Untersuchung von Creep/Recoverykurven . . . . . . . . . . . . . . 63

3.1.4 Creep/Recoverymessungen an amorphem PdCuSi zur Untersuchung von temperatur- und spannungsabhängigem Elastizitätsverhalten ......................... 70

3.1.5 Creep/Recoverymessungen an amorphem ZrAlCu zur Untersuchung von temperatur- und spannungsabhängigem Elastizitätsverhalten . . . . . . . . . . . . . . 81

3.2 Relaxationsprozesse von ABA-Block-Copolymeren . . . . . . . . . . . . 89

4 Diskussion $\quad 101$

4.1 Relaxationen metallischer Gläser . . . . . . . . . . . . . . . . . . . . 101

4.1.1 Heizratenabhängige Relaxationsprozesse von amorphem $\mathrm{PdCuSi}$ unterhalb der Glasübergangstemperatur . . . . . . . . . . . . 101

4.1.2 Einfluss von Kraftänderungen auf das mechanische Verhalten von amorphem PdCuSi bei Spannungs-Dehnungskurven . . . . . . . . 110

4.1.3 Spannungsabhängiges Dämpfungsverhalten von PdCuSi . . . . . . 115

4.1.4 Glasübergangstemperatur und Aktivierungsvolumen von $\mathrm{PdCuSi}$ in Abhängigkeit der äußeren Spannung . . . . . . . . . . . . . . . 123 
4.1.5 Glasübergangstemperatur und Aktivierungsvolumen von $\mathrm{ZrAlCu}$ in Abhängigkeit der äußeren Spannung . . . . . . . . . . . . . . 127

4.2 Relaxationen von wasserstoffbrückenbildenden Block-Copolymeren . . . . 130

5 Zusammenfassung

135

6 Anhang

Literatur 



\section{Abbildungsverzeichnis}

1.1 a) Darstellung der Volumen- bzw. Enthalpieänderungen beim Kühlen aus der Schmelze, ${ }^{[8]}$ b) zugehörige Änderung in der spezifischen Wärme. ${ }^{[9]}$. . 3

1.2 Abhängigkeit der Glastemperatur von der Abkühlrate $\bar{T}\left(\bar{T}_{3}>\bar{T}_{2}>\bar{T}_{1}\right)^{[9]} 4$

1.3 Angell-Plot: Temperaturabhängigkeit der Viskosität oberhalb der Glastemperatur $^{[17]} \ldots \ldots \ldots \ldots \ldots \ldots$

1.4 Bestimmung des Phasenwinkels . . . . . . . . . . . . . 8

1.5 Spannungs-Dehnungskurve bei konstanter Dehnungsrate . . . . . . . . . 9

1.6 Veränderung des Probenquerschnitts bei Spannungs-Dehnungskurven modifiziert nach Föll ${ }^{[20]} \ldots \ldots \ldots$. . . . . . . . . . . . 10

1.7 Spannungs-Dehnungskurve mit konstanter Krafterhöhung . . . . . . . . . 11

1.8 Creep/Recoverymessung zur Bestimmung der Delay-time . . . . . . . . . 12

1.9 Lunkenheimer-Loidl-Plot: Schematische Darstellung des durch Relaxationen hervorgerufenen Verlustspektrums eines amorphen Materials ${ }^{[32]}$. . . 15

1.10 Slow $\beta$ - Relaxation in einem starken metallischen Glasbildner ${ }^{[13]}$. . . . . 16

1.11 Slow $\beta$ - Relaxation in einem fragilen metallischen Glasbildner ${ }^{[14]}$. . . . . 17

1.12 Kettenartige, quadrupolförmige Anregung der slow $\beta$-Relaxation ${ }^{[43]}$. . . 18

1.13 Schematische Darstellung eines kollektiven, kettenartigen $\beta$ - Prozesses mit zugehörigem Spannungsfeld (rote Linie) ${ }^{[45]}$. . . . . . . . . . . . . . . 19

1.14 Verschwinden des durch $\beta$ - Relaxation induzierten Verlustprozesses ab einer Schichtdicke von $25 \mathrm{~nm}^{[45]} \ldots \ldots$. . . . . . . . . . . . 19

1.15 Durch Scherung angeregte lokalen STZs. Die blauen Cluster geben die Verschiebungen der Atome nach der Scherung wieder. ${ }^{[46]}$. . . . . . . . . 20

1.16 Relaxationsprozesse im Bild einer potenziellen Energielandschaft ${ }^{[50]}$. . . 22

1.17 Effekt der thermischen und mechanischen Vorbehandlung auf die Elastizitätsmodule von a) $\mathrm{PdCuSi}$ und b) $\mathrm{ZrAlCu}^{[14]} \ldots \ldots$. . . . . . . . . . 23 
1.18 Alterung durch Auslagerungsexperimente ${ }^{[61]}$. . . . . . . . . . . . . 24

1.19 Alterung der Probe bis ca. $450 \mathrm{~K}$ durch thermisches Anlassen beim

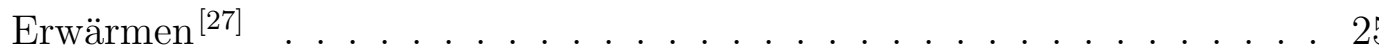

1.20 Spannungs-Dehnungskurven von amorphem $\mathrm{Pd}_{43} \mathrm{Ni}_{10} \mathrm{Cu}_{27} \mathrm{P}_{20}$ bei $548 \mathrm{~K}$ $\left(\mathrm{T}_{G}=570 \mathrm{~K}\right)$ mit einer Dehnungsrate von $10^{-4} \mathrm{~s}^{-1}$. Sechs verschiedene

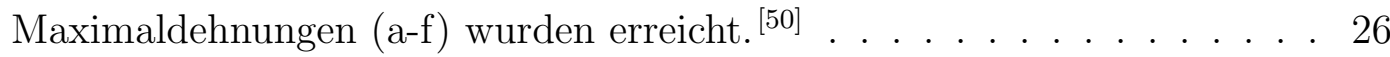

1.21 Frenkelansatz zur Erniedrigung der Barrierenhöhe zwischen zwei Metabasins innerhalb der PEL ${ }^{[47]} \ldots \ldots$. . . . . . . . . . . . 27

1.22 Temperaturabhängige Relaxationsprozesse von Polymeren ${ }^{[18]}$. . . . . . . 30

1.23 Kurbelwellenbewegung nach a) Schatzki und b) Boyer ${ }^{[74,75]}$. . . . . . . . 31

1.24 Verwendete Monomereinheiten bei der Untersuchung des Chemical Con-

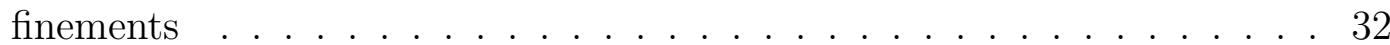

1.25 Zusätzlicher Chemical Confinement Peak unterhalb von $\mathrm{T}_{G}{ }^{[14]} \ldots$. . . . 33

1.26 Schematische Darstellung der Idee des Chemical Confinements: a) statistisches Polymer mit a') Verteilung der möglichen Wasserstoffbrücken, b) blockweise aufgebautes Polymer mit b') Wasserstoffbrücken, die ein Chemical Confinement bilden. ${ }^{[14]} \ldots \ldots \ldots \ldots$

2.1 Schematische Abbildung der DMA mit Vergrößerung der Probenaufhängung zur Vermessung der metallischen Bänder ${ }^{[79]}$. . . . . . . . . . . 37

2.2 Verwendetes RAFT-Agenz zur Herstellung der Block-Copolymere . . . . 40

2.3 Verwendete Monomereinheiten . . . . . . . . . . . . . . . . . . . . 41

2.4 Schematische Abbildung der ABA-Block-Copolymere . . . . . . . . . . . 42

2.5 Probenauflage zur Vermessung der Polymerproben . . . . . . . . . . . . . 43

3.1 Vergleich von a) Speicher- und b) Verlustmodul bei verschiedenen Heizraten 46

3.2 Vergleich der Moduli vom a) ersten, b) zweiten, c) dritten und d) vierten Durchlauf der Untersuchung des Relaxationsverhaltens bei einer Heizrate von $0,1 \mathrm{~K} / \mathrm{min} \ldots \ldots \ldots \ldots \ldots$. . . . . . . . . . . . . . . . . . . 48

3.3 Vergleich der Probenlänge der vier Durchläufe . . . . . . . . . . . . . . . 49

3.4 Gegenüberstellung der Relaxationsvorgänge des vierten Durchlaufs mit der kristallisierten Probe . . . . . . . . . . . . . . . . . . 50 
3.5 Vergleich des normierten Wärmeflusses vom ersten (schwarz) und zweiten (rot) Durchlauf bei einer Heizrate von $0,1 \mathrm{~K} / \mathrm{min}$. Die blauen Linien sind über 5 Datenpunkte geglättete Kurven. . . . . . . . . . . . . . . . . . 51

3.6 Temperaturverlauf zur Untersuchung der Elastizitätsmodule bei Erwärmung und Abkühlung der Probe . . . . . . . . . . . . . . 52

3.7 Verlustmodul beim Erwärmen und Abkühlen der Probe, der erste Zyklus $(1 / 2)$ ist in schwarz, der zweite $(3 / 4)$ in blau und der dritte $(5 / 6)$ in rot dargestellt. . . . . . . . . . . . . . . . . . 5 53

3.8 Speichermodul beim Erwärmung und Abkühlung der Probe . . . . . . . . 54

3.9 Spannungs-Dehnungskurven von Haaren bei einer Kraftänderung von $10 \mathrm{mN} / \mathrm{min}$ (schwarz) und $1 \mathrm{mN} / \mathrm{min}$ (rot). In blau sind die Verlängerungen der linearen Bereiche eingezeichnet . . . . . . . . . . . . . 55

3.10 Spannungs-Dehnungskurven von PdCuSi bei $640 \mathrm{~K}$ mit zwei unterschiedlichen Kraftänderungen . . . . . . . . . . . . . . . . . . 56

3.11 Vergleich der Spannungs-Dehnungskurven von PdCuSi mit einer Kraftänderung von $5 \mathrm{mN} / \mathrm{min}$ bei verschiedenen Temperaturen . . . . . . . . . . 57

3.12 Bestimmung der Elastizitätsmodule der Spannungs-Dehnungskurven aus Abb. 3.11 in linearer Auftragung im Bildteil a) und logarithmischer im Bildteil b). . . . . . . . . . . . . . . . . . . 58

3.13 Vergleich der Spannungs-Dehnungskurven von PdCuSi mit einer Kraftänderung von a) $300 \mathrm{mN} / \mathrm{min}$ und b) $-300 \mathrm{mN} / \mathrm{min} \ldots$. . . . . . . . . . 59

3.14 Bestimmung der Elastizitätsmodule der Spannungs-Dehnungskurven aus Abb. 3.11, die schwarzen Punkte sind die Werte zugehörig zur positiven, die roten zur negativen Kraftänderung . . . . . . . . . . . . . 60

3.15 Spannungs-Dehnungskurve von PdCuSi bei Raumtemperatur mit einer Dehnungsrate von $1 \cdot 10^{-7} \mathrm{~s}^{-1} \ldots \ldots \ldots 61$

3.16 Vergleich der Probendehnung (blau) mit der aufgenommenen Kraftänderung (schwarz). Die rote Linie zeigt eine Glättung der Kraft über 10 Datenpunkte. 
3.17 Creep/Recoverykurven von a) einer kristallisierten und b) einer amorphen PdCuSi Probe bei 570 K. Die Kräfte betrugen $450 \mathrm{mN}$ und $50 \mathrm{mN}$. Zu sehen ist die Probendehnung (blau) bzw. die verwendeten Kräfte (schwarz) gegenüber der Versuchszeit bei vier Wiederholungen der Kraftsprünge. 63

3.18 Delay-time vom amorphen PdCuSi im Vergleich zu kristallisierten Proben 64

3.19 Berechneter Elastizitätsmodul aus den Creep/Recoverymessungen . . . . 65

3.20 Vergleich der Delay-time bei 520 K mit verschiedenen Kräften. Die untere Kraft beim Zurückspringen wurde mit $50 \mathrm{mN}$ konstant gehalten und nur die obere, maximale Kraft wurde systematisch erhöht. . . . . . . . . . . . 66

3.21 Vergleich der Delay-time zwischen Creep- und Recoveryteil . . . . . . . . 67

3.22 Spannungs- und Temperaturabhängigkeit der Delay-time (Dämpfung), welche als schwarze Punkte abgebildet und über ein dreidimensionales Netz miteinander verbunden wurde. In der x-y Ebene ist die Veränderung in Abständen von 2 in der Delay-time (rote Linien im Gitternetz) farbig projeziert. Die Farblegende ist links neben der Auftragung zu sehen. . . . 68

3.23 Messkurve einer Creep/Recoverymessung von PdCuSi mit den Kräften $450 \mathrm{mN}$ und $50 \mathrm{mN}$ bei einer Heizrate von $0,78 \mathrm{~K} / \mathrm{min}$. Der Inset zeigt eine Vergrößerung des Temperaturbereichs von 422 K bis 428 K. . . . . . 70

3.24 Aus Creep/Recoverymessungen bestimmter Elastizitätsmodul von $\mathrm{PdCuSi}$ von Raumtemperatur bis $840 \mathrm{~K} \ldots \ldots . . . . . . . . .71$

3.25 Vergleich unterschiedlicher Creep/Recoverymessungen von vier Modulberechnungen mit a) $450 \mathrm{mN}(10 \mathrm{MPa}$ - schwarz) und $50 \mathrm{mN}$ (1 MPa - rot), b) $500 \mathrm{mN}(8,8 \mathrm{MPa}$ - schwarz) und $100 \mathrm{mN}(1,8 \mathrm{MPa}$ - rot), c) $650 \mathrm{mN}$ (16,0 MPa - schwarz) und $250 \mathrm{mN}(6,1 \mathrm{MPa}$ - rot) und d) $750 \mathrm{mN}(21,2$ $\mathrm{MPa}-$ schwarz $)$ und $350 \mathrm{mN}(9,9 \mathrm{MPa}-$ rot $) \ldots \ldots . . \ldots 72$

3.26 Spannungsabhängige Glasübergangstemperatur von $\mathrm{PdCuSi}$. . . . . . . 74

3.27 Berechnete Dehnungsratensensitivität zur in Abbildung 3.25 a) dargestellten Messung mit den verwendeten Kräften $450 \mathrm{mN}$ (schwarz) und 50

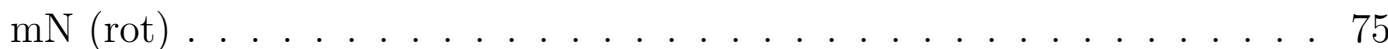

3.28 Berechnetes Aktivierungsvolumen zur in Abbildung 3.25 a) dargestellten Messung mit den verwendeten Kräften $450 \mathrm{mN}$ (schwarz) und $50 \mathrm{mN}$ (rot). 76 
3.29 Maximal bestimmtes Aktivierungsvolumen und die zugehörige Temperatur in Abhängigkeit der äußeren Spannung . . . . . . . . . . . . . . 77

3.30 Wahre Dehnung gegenüber der berechneten Dehnungsrate zur Bestimmung des Aktivierungsvolumens in der unterkühlten Schmelze . . . . . . 78

3.31 Halblogarithmische Auftragung der Dehnungsrate bei konstanter Dehnung gegenüber der wahren Spannung zur Bestimmung des Aktivierungsvolumens in der unterkühlten Schmelze. . . . . . . . . . . . . . . . . . . . 79

3.32 Aktivierungsvolumen in Abhängigkeit der wahren Dehnung in der unterkühlten Schmelze . . . . . . . . . . . . . . . . . . . . 80

3.33 Relative Probenlänge gegenüber der Probentemperatur bzw. der Versuchszeit bei den verwendeten Kräften von $500 \mathrm{mN}$ und $100 \mathrm{mN}$. . . . . 81

3.34 Vergleich von zwei Modulberechnungen von $\mathrm{ZrAlCu}$ a) $500 \mathrm{mN}$ (4,9 MPa - schwarz) und $100 \mathrm{mN}(1,0 \mathrm{MPa}$ - rot), b) $650 \mathrm{mN}$ (10,8 MPa - schwarz) und $250 \mathrm{mN}(4,2 \mathrm{MPa}-\mathrm{rot}) \ldots \ldots \ldots$. . . . . . . . . . . . 82

3.35 Spannungsabhängige Glasübergangstemperatur von $\mathrm{ZrAlCu}$. . . . . . . 83

3.36 Aktivierungsvolumen von $\mathrm{ZrAlCu}$ im Temperaturbereich von $610 \mathrm{~K}$ bis $680 \mathrm{~K}$ bei den verwendeten Kräften $500 \mathrm{mN}$ (4,9 MPa - schwarz) und $100 \mathrm{mN}(1,0 \mathrm{MPa}-\mathrm{rot}) \ldots \ldots . \ldots . \ldots . \ldots 84$

3.37 Maximal bestimmtes Aktivierungsvolumen von $\mathrm{ZrAlCu}$ und die zugehörige Temperatur in Abhängigkeit der äußeren Spannung . . . . . . . . . . . . 85

3.38 Halblogarithmische Auftragung der Dehnungsrate bei konstanter Dehnung gegenüber der wahren Spannung für $\mathrm{ZrAlCu}$ Proben . . . . . . . . . . . . 86

3.39 Aktivierungsvolumen in Abhängigkeit der wahren Dehnung in der unter-

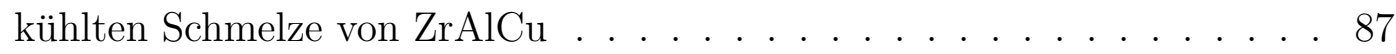

3.40 Vergleich von zwei DSC Messungen a) B-Block 5504g/mol, A-Block $11280 \mathrm{~g} / \mathrm{mol}$ und b) B-Block $9528 \mathrm{~g} / \mathrm{mol}$, A-Block $9390 \mathrm{~g} / \mathrm{mol}$. . . . . . . 89

3.41 DSC Messung der Probe aus 3.40 b) nach Auslagerung bei $400 \mathrm{~K}$ für 30 min. . . . . . . . . . . . . . . . . . . . 90

3.42 Glasübergangstemperatur aus DSC-Messungen bestimmt von unbehandelten Proben (schwarz) und einer bei $400 \mathrm{~K}$ für 30 min ausgelagerten Probe in Abhängigkeit des inneren B-Blockes. . . . . . . . . . . . . . . . 91 
3.43 Vergleich der Röntgenspektren von ABA-Block-Copolymeren unbehandelt (schwarz) und nach Auslagerung für 30 min bei $400 \mathrm{~K}$ (rot). . . . . . . . 92

3.44 Vergleich der Relaxationsspektren von drei verschiedenen ABA-BlockCopolymeren (die Molekulargewichte der einzelnen Blöcke sind in der Abbildung genannt).

3.45 Vergleich des Relaxationspeaks bei $\mathrm{T}_{G}$ bei gleicher Länge des inneren Blockes B (die Molekulargewichte der einzelnen Blöcke sind in der Abbildung genannt). . . . . . . . . . . . . . . . . . . 9

3.46 Vergleich der gemessenen Probenhöhe mit dem Relaxationsspektrum einer Polymerprobe mit einem B-Block von $3472 \mathrm{~g} / \mathrm{mol}$ und einem A-Block von $12800 \mathrm{~g} / \mathrm{mol}$.

3.47 Vergleich der gemessenen Probenhöhe von vier verschiedenen ABA-BlockCopolymeren (die Molekulargewichte der einzelnen Blöcke sind in der Abbildung genannt). . . . . . . . . . . . . . . . 95

3.48 Vergleich der gemessenen Probenhöhe eines ABA-Block-Copolymers mit einer reinen $\mathrm{p} t \mathrm{BA}$ Probe (die Molekulargewichte der einzelnen Blöcke sind in der Abbildung genannt). . . . . . . . . . . . . . . . . . 96

3.49 Vergleich der Glasübergangstemperatur bestimmt aus der gemessenen Probenhöhe a) und den Relaxationsspektren b) in Abhängigkeit des inneren B-Blockes . . . . . . . . . . . . . . . . . 97

3.50 Vergleich der Änderung in der Probenposition oberhalb von $\mathrm{T}_{G}$ in Abhängigkeit der äußeren A-Blöcke. . . . . . . . . . . . . . . . . 98

3.51 Vergleich der sekundären Relaxation unterhalb von $\mathrm{T}_{G}$ bei gleicher Länge des inneren Blockes B (die Molekulargewichte der einzelnen Blöcke sind in der Abbildung genannt). . . . . . . . . . . . . . . . 999

3.52 Temperatur des Maximums der sekundären Relaxation unterhalb von $\mathrm{T}_{G}$ in Abhängigkeit des inneren B-Blocks.

4.1 Abhängigkeit der Glasübergangstemperatur von der Heizrate . . . . . . . 102

4.2 Abhängigkeit der Steigung des Wings von der Heizrate unterhalb der Glasübergangstemperatur . . . . . . . . . . . . . . . . 103 
4.3 Dämpfungsmessung mittels DPO an einem amorphen $\mathrm{Pd}_{77,5} \mathrm{Cu}_{6,0} \mathrm{Si}_{16,5^{-}}$ Film mit einer Schichtdicke von $300 \mathrm{~nm}$, bei einer Frequenz von 4,6 kHz und einer Heizrate von 0,1 K/min. Der Schermodul liegt nahe Raumtemperatur bei 36 GPa. $^{1}$. . . . . . . . . . . . . . . . . . . . . 104

4.4 Einordnung der Relaxationen der langsamen Heizrate (Quadrate mit schwarzem Rand) in eine Kissingerauftragung zum Mergingverhalten nach Hachenberg. ${ }^{[85]}$ In rot ist der Onset und in blau der Wendepunkt des Speichermoduls, sowie in grün der Onset der Probenlänge aufgetragen. Die runden Datenpunkte wurden von Hachenberg et al. ${ }^{[85]}$ übernommen. Alle Messpunkte wurden mit einem linearen Fit extrapoliert. . . . . . . . 106

4.5 Abhängigkeit der potentiellen Energie von einer generalisierten Koordinate nach Harmon ${ }^{[50]}$. Die Energie zum Erreichen eines höheren Subbasins innerhalb der PEL (E2) ist größer als die zur Alterung benötigte (E1). . 108

4.6 Abhängigkeit des Überganges von homogener zu nichthomogener Deformation und vom Newtonischen zum nicht-Newtonischen Fließen über die Änderung der Dehnungsrate bzw. der Temperatur von amorphem $\mathrm{Zr}_{41,2} \mathrm{Ti}_{13,8} \mathrm{Cu}_{12,5} \mathrm{Ni}_{10,0} \mathrm{Be}_{22,5}{ }^{[103]} \ldots \ldots \ldots 111 \ldots \ldots \ldots$

4.7 Schematische Abnahme der Barrierenhöhe der PEL durch eine äußere Spannung $\sigma_{0}<\sigma_{1}<\sigma_{2}<\sigma_{3} \ldots \ldots \ldots \ldots 116$

4.8 Exponentielle Anpassung der Dämpfungslandschaft nach Messung aus

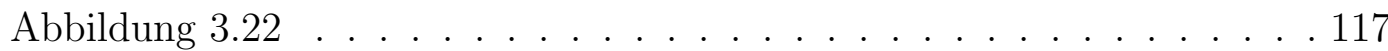

4.9 Vergleich der berechneten inversen Delay-time (schwarze Punkte) gegenüber den Messwerten (rote Punkte) aufgetragen gegen die inverse Temperatur. Die graue bzw. rote Kurve entspricht einem Vogel-FulcherTammann-Verhalten. . . . . . . . . . . . . . . . . . . . . . 119

4.10 Vergleich verschiedener Spannungen zur Nachprüfung des Vogel-FulcherTammann-Verhaltens der Delay-time. Die Kurven wurden mit der Gleichung 4.1 berechnet, in grau ist das VFT-Verhalten vergleichend eingezeichnet. . . . . . . . . . . . . . . . . 120

4.11 Vergleich der inversen Delay-time gegenüber der äußeren Spannung bei verschiedenen Temperaturen. Die Kurven wurden über die Gleichung 4.1 berechnet. . . . . . . . . . . . . . . . . . . . 121 


\section{Einleitung}

\subsection{Glas}

In der Entwicklungsgeschichte des Menschen hat Glas eine wichtige Bedeutung. Der abgeleitete Name vom germanischen glasa (das Glänzende, Schimmernde) gibt schon einen Hinweis auf die ungewöhnlichen physikalischen Eigenschaften, die wichtig für die Benutzung von Gläsern sind.

Natürliche Gläser wie Obsidian $\left(\mathrm{SiO}_{2}+\mathrm{H}_{2} \mathrm{O}+\mathrm{Fe}_{2} \mathrm{O}_{3}+\mathrm{Al}\right.$,C,Ca) konnten schon in der Steinzeit aufgrund ihrer scharfen und harten Bruchstellen als Werkzeuge benutzt werden. Die kommerzielle Verwendung jedoch begann erst in der Antike um 1600 v. Chr., als Gläser wie Perlen als Schmuck verwendet wurden. Sie waren höchstwahrscheinlich schon damals ein Zeichen von Reichtum und gesellschaftlicher Stellung. Die Verarbeitung zu nutzbaren Kelchen ist etwa 1450 v. Chr. entstanden. So wurden die ersten Trinkgefäße aus Glas in Ägypten verwendet (das älteste gefundene Exemplar trägt den Namen vom Pharao Thutmosis III und zeigt somit auch die elitäre Stellung der Gläser auf). In der Antike waren Gläser ein wichtiges Handelsgut, was große Schmelzbetriebe und Funde in Schiffwracks vermuten lassen.

Im Mittelalter und in der Neuzeit entwickelte sich die Kunst des Glasblasens. So konnte das Glas gezielt in Form gebracht werden und wurde ein wichtiges Gut für Fenster, vor allem in Kirchen und adligen Häusern. ${ }^{[1-3]}$

In Venedig wurde die Glasherstellung im 13. Jahrhundert zu einem wichtigen Industriezweig, welcher sich mit dem Muranoglas bis heute hält.

Im 18. Jahrhundert wurde eine Neuerung in der Glasherstellung wichtig. Durch die Herstellung von geschnittenem und geschliffenem Glas aus Deutschland konnten den Formen Zusatzstoffe wie Metallfüße oder Goldränder und -motive angefügt werden. Diese wurden vor allem für herrschaftliche Anlässe angefertigt. 
Die großindustriellen Möglichkeiten, Glas herzustellen, führten dazu, dass das Glas aus dem heutigen Leben nicht mehr wegzudenken ist. Alle Erneuerungen in der Glasherstellung hatten zur Folge, dass Gläser vielseitiger und gezielter einzusetzen waren. Der Fortschritt hing dabei immer mit einem Wissensgewinn über das Material zusammen. Heute wird bei Gläsern zwischen vielen möglichen Materialien unterschieden. So ist es nicht nur möglich, Gläser aus Siliziumoxid herzustellen (Fensterglas), sondern auch aus Polymeren und Metallen. Alle diese verschiedenen Materialien machen es möglich, dass Gläser einen sehr weiten Anwendungsbereich finden. ${ }^{[1-4]}$

In der Physik wird allgemein von Glas gesprochen, wenn eine Substanz im festen, amorphen Zustand ist und einen Glasübergang zeigt. Im Gegensatz zu kristallinen Materialien gibt es keine Fernordnung mit festen Gitterplätzen, sondern eine Nahordnung. Das Glas zeigt in den Atompositionen eine sehr enge Verwandtschaft zu Flüssigkeiten (siehe Kapitel 1.1.2). Dabei gibt es viele verschiedene Möglichkeiten, ein Glas herzustellen, wie z. B. über das schnelle Abkühlen der Flüssigkeit, die Erwärmung von Sol-Gelen oder die Zerstörung der kristallinen Struktur über Stoßwellen. ${ }^{[5]}$

\subsubsection{Physik des Glasübergangs}

Die Physik des Glasüberganges ist bis heute in der Diskussion und nicht vollständig geklärt. ${ }^{[6]}$ In den letzten Jahren wurden viele neue Erkenntnisse durch molekulardynamische Simulationen und Spektroskopie gewonnen, ${ }^{[7]}$ die die Physik des Glasüberganges besser charakterisieren. Der Übergang vom flüssigen zum festen Zustand wurde in der Literatur ausführlich beschrieben, ${ }^{[4-7]}$ so dass dies in dieser Arbeit nur sehr kurz erläutert wird. Nachfolgend sollen nun die wichtigsten Theorien zu inneren Relaxationsvorgängen in Gläsern vorgestellt und eine Einführung in die spektroskopischen Grundlagen der Gläser gegeben werden. 


\subsubsection{Unterschied zwischen Gläsern und Flüssigkeiten}

Gläser und Flüssigkeiten ähneln sich sehr stark, da sich die Atompositionen beim Glasübergang nicht sprunghaft ändern, sondern die Flüssigkeit „eingefroren“ wird. Durch zwei Parameter (Relaxationszeit und Beobachtungszeitskala) können Gläser und Flüssigkeiten jedoch klar unterschieden werden. Eine sehr gute Möglichkeit bietet hierfür die Deborah Zahl DE.

$$
D E=\frac{\tau}{t_{c h a r}}
$$

Man unterscheidet eine Flüssigkeit von einem Glas über das Verhältnis der Relaxationszeit $\tau$ des Materials zur charakteristischen Beobachtungszeit $\mathrm{t}_{\text {char }}$. Je kleiner das Verhältnis, desto flüssiger ist das Material. Jedoch ist auch in dieser Beschreibung der Übergang von flüssig zu fest kontinuierlich.

Per Definition ist ein Glas ein amorpher Festkörper, der einen Glasübergang zeigt. ${ }^{[8]}$ Amorph beschreibt dabei, dass die Atompositionen im Glas keine Fernordnung besitzen. Röntgenspektren können dies belegen und wurden in der Literatur häufig diskutiert. ${ }^{[8]}$
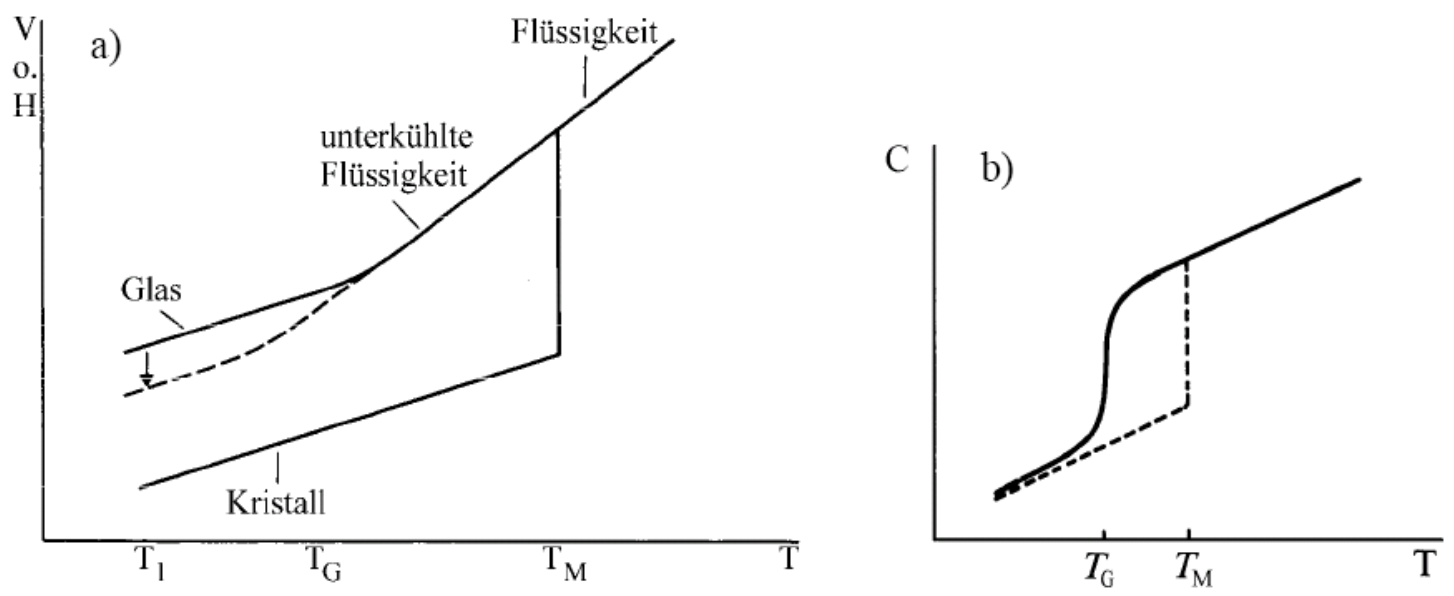

Abbildung 1.1: a) Darstellung der Volumen- bzw. Enthalpieänderungen beim Kühlen aus der Schmelze, ${ }^{[8]}$ b) zugehörige Änderung in der spezifischen Wärme. ${ }^{[9]}$

Eine Möglichkeit, den Glasübergang zu erkennen, bieten Messungen des Volumens oder der spezifischen Wärme. Wie in Abbildung 1.1 skizziert ist, verändert sich bei der Glasübergangstemperatur $\mathrm{T}_{G}$ die Steigung der Volumenänderung (a). In der spezifischen Wärme ist ein S-förmiger Sprung zu sehen (b). Das Abknicken ist nicht scharf wie bei der 
Kristallisation $\mathrm{T}_{M}$, da es sich nicht um einen Phasenübergang erster Ordnung handelt, sondern sich die Relaxationszeit aus vielen Einzelprozessen zusammensetzt. ${ }^{[10]}$ Dieser Übergang ist jedoch im Detail noch nicht vollständig verstanden.

Beim sehr schnellen Abkühlen der Flüssigkeit und der Unterdrückung der Kristallisation erreicht man den Bereich der unterkühlten Schmelze (siehe Abb. 1.1 und 1.2 Bereich B). Durch verschiedene Abkühlraten findet der Übergang aus diesem metastabilen Zustand bei verschiedenen Temperaturen statt, wie in Abbildung $1.2 \mathrm{zu}$ sehen ist. Dies ist kinetisch zu erklären, da die Atome bei schnellerer Abkühlrate nicht genügend Zeit haben, um in eine energetisch günstigere Konfiguration zu gelangen. Die Glastemperatur ist also unter anderem kühlratenabhängig und keine universelle Materialkonstante.

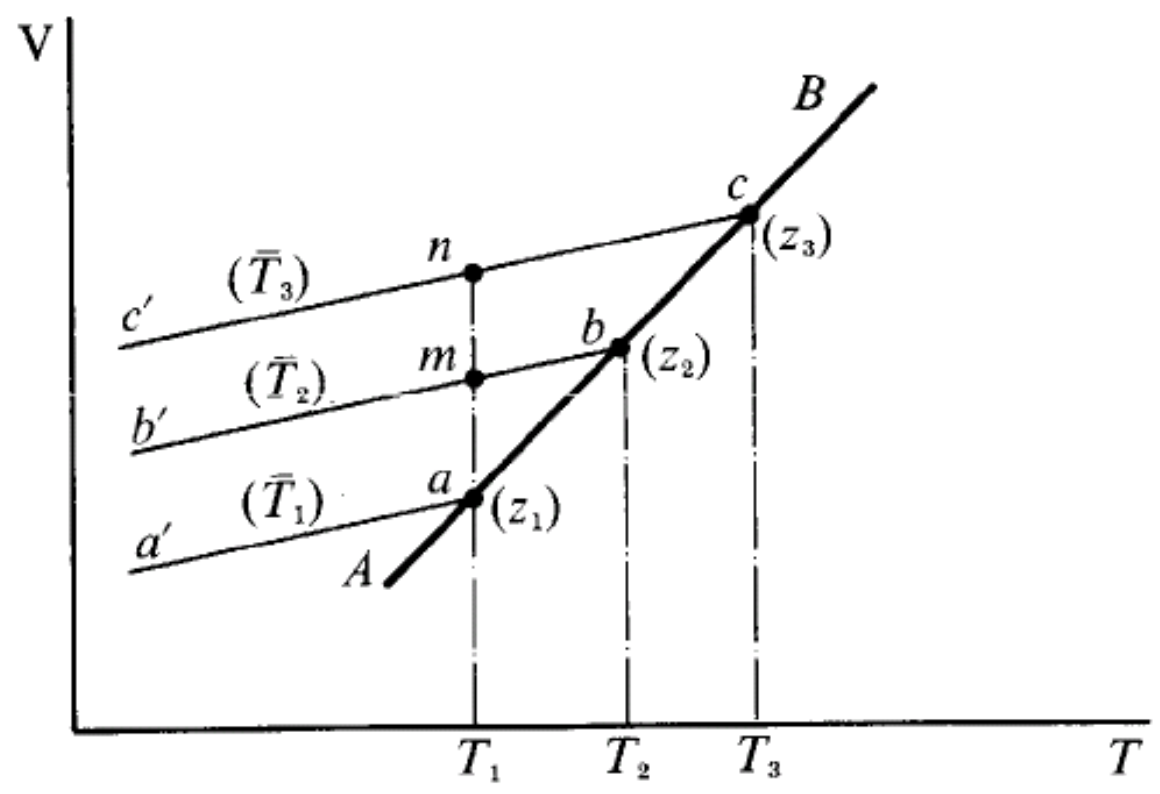

Abbildung 1.2: Abhängigkeit der Glastemperatur von der Abkühlrate $\bar{T}$ $\left(\bar{T}_{3}>\bar{T}_{2}>\bar{T}_{1}\right)^{[9]}$ 


\subsection{Grundlagen der Viskoelastizität}

Amorphe Festkörper besitzen im Gegensatz zu kristallinen, wie im Kapitel 1.1.2 bereits erwähnt, keinen scharf zu messenden Schmelzpunkt, sondern erweichen in einem kontinuierlichen Übergang. Dementsprechend sind die makroskopischen Eigenschaften eines Glases dadurch bestimmt, wie nah es an dem Erweichungspunkt (dem Glasübergang) liegt. Das Glas verhält sich bei tiefen Temperaturen auf eine äußere angelegte Kraft bei der Deformation überwiegend elastisch wie ein Kristall. Je näher man dem Glasübergang kommt, desto nachgiebiger wird das Material und die Dämpfung einer mechanischen Anregung nimmt zu. Es wird viskos und flüssig. In der Nähe des Glasüberganges wird das Material als viskoelastisch bezeichnet, da es sowohl den Charakter einer viskosen Flüssigkeit als auch den eines elastischen Festkörpers vereint. Da dies eine besondere Eigenschaft von glasbildenden Systemen ist, welche mikroskopisch noch nicht vollständig geklärt ist, soll das Kapitel 1.2 hierzu eine kurze Einführung geben, da in dieser Arbeit der nichtlineare Übergang vom elastischen zum inelastischen viskosen Verhalten untersucht wird.

Die physikalischen Gesetzmäßigkeiten wurden in der Literatur ausführlich dargestellt, ${ }^{[9,11-16]}$ deshalb werden nur die wichtigsten in dieser Arbeit verwendeten Gleichungen beschrieben.

Gläser werden üblicherweise in zwei verschiedene Klassen eingeteilt (starke und fragile Glasbildner). Man unterscheidet sie über ihre Viskosität, welche auf einer logarithmierten Skala in Abhängigkeit der normierten, inversen Temperatur aufgetragen wird. In Abbildung 1.3 ist für verschiedene Glasbildner ein Vergleich aufgestellt. Es wird die Glasübergangstemperatur bei einer Viskosität von $10^{12} \mathrm{~Pa} \cdot \mathrm{s}$ festgelegt, wodurch ein Schnittpunkt aller Verläufe erzeugt werden kann. Die Unterscheidung zwischen starken und fragilen Glasbildnern wird über die Steigung in diesem Punkt festgelegt (sogenannte Fragilität). Für starke Glasbildner beschreibt ein Arrhenius-Gesetz den Verlauf der Viskosität (linearer Verlauf in dieser Abbildung),

$$
\eta=\eta_{0} \cdot \exp \left(\frac{E_{A}}{k_{B} T}\right)
$$

wobei $\eta$ die Viskosität, $\mathrm{E}_{A}$ die Aktivierungsenergie, $\mathrm{k}_{B}$ die Boltzmann-Konstante und $\mathrm{T}$ die absolute Temperatur darstellen. 
Für die fragilen Glasbildner beschreibt ein Vogel-Fulcher-Tammann-Gesetz den Verlauf besser, ${ }^{[28-30]}$

$$
\eta=\eta_{0} \cdot \exp \left(\frac{D \cdot T_{V F T}}{T-T_{V F T}}\right)
$$

wobei $\mathrm{D}$ als Stärkeparameter und $\mathrm{T}_{V F T}$ als Vogel-Fulcher-Tammann-Temperatur bezeichnet wird. Hierbei muss jedoch angemerkt werden, dass in neueren Studien die Theorie des Vogel-Fulcher-Tammann-Gesetzes als beste Möglichkeit zur Beschreibung der viskosen Dynamik angezweifelt wird. ${ }^{[31]}$

Um Gesetzmäßigkeiten für glasbildende Systeme zu definieren, müssen immer sowohl fragile als auch starke Glasbildner untersucht werden, um die verschiedenen viskoelastischen Dynamiken zu berücksichtigen.

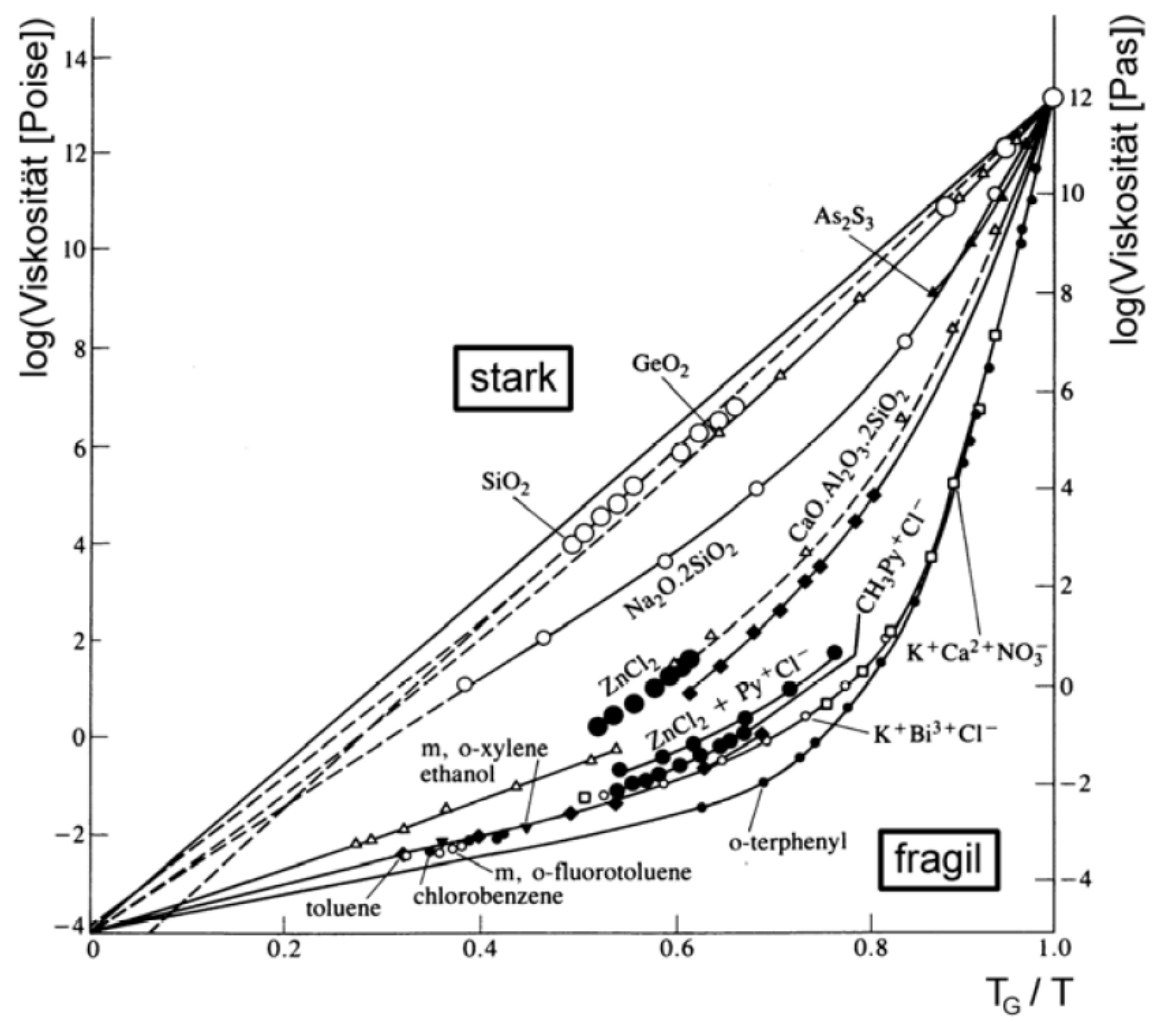

Abbildung 1.3: Angell-Plot: Temperaturabhängigkeit der Viskosität oberhalb der Glastemperatur ${ }^{[17]}$ 


\subsection{Dynamisch-mechanische Messungen an viskoelastischen Materialien}

In diesem Kapitel werden verschiedene mechanische Spektroskopiearten vorgestellt, mit denen Relaxationen in amorphen Festkörpern untersucht werden können. Dabei ist der Fokus auf die dynamisch-mechanische Analyse der Festkörper gerichtet (Abschnitt 1.3.1). Des Weiteren werden die für Vergleichsmessungen verwendeten spektroskopischen Methoden erläutert (Abschnitt 1.3.2) und die wichtigsten Gleichungen der Messarten hierfür vorgestellt und erklärt.

\subsubsection{DMA}

Die dynamisch-mechanische Analyse (DMA) dient der Bestimmung der viskoelastischen Eigenschaften einer makroskopischen Probe. Hierbei können verschiedene Messmodi verwendet werden. In den folgenden Abschnitten werden die für diese Arbeit wichtigen Messarten eingeführt und die zugehörigen Gleichungen beschrieben. Die Messmethode wird im Kapitel 2 ausführlich erklärt.

\subsubsection{Messung mit einer dynamischen Kraft}

Allgemein wird bei der dynamisch-mechanischen Spektroskopie eine statische Spannung $\sigma_{s t a}$ von einer sinusförmigen, dynamischen $\sigma_{d y n}$ überlagert, weshalb eine komplexe Schreibweise bei der Berechnung von Materialkonstanten vorteilhaft ist (folgend mit * bezeichnet).

Durch die externe Spannung wird das Material ausgelenkt (Antwortfunktion) und folgt dieser. Dabei wird die Dehnung des Materials $\epsilon$ gegenüber der Zeit t beobachtet. Da das Material aufgrund von Verlustprozessen mit einer Verzögerung auf die Kraft reagiert (Dämpfung, interne Reibung), kann man die zeitliche Verzögerung in einem Phasenwinkel $\delta$ ausdrücken. Dieser ist ein Maß für die Dämpfung und kann zur Berechnung von Materialkonstanten verwendet werden. ${ }^{[9,18]}$

$$
\sigma_{d y n}^{*}(t)=\sigma_{0} \cdot \exp (i \omega t)
$$


Die um $\delta$ Phasen verschobene Antwort berechnet sich nach

$$
\epsilon_{d y n}^{*}(t)=\epsilon_{0} \cdot \exp (i \omega t-i \delta)
$$

Dabei ergibt sich bei einer oszillierenden, erzwungenen Dehnung

$$
\sigma_{d y n}^{*}(t)=E^{*} \cdot \epsilon=\left(E^{\prime}+i E^{\prime \prime}\right) \epsilon_{d y n}^{*}
$$

wobei E als Young- oder Elastizitätsmodul bezeichnet wird, E' der reale Speichermodul und E" der komplexe Verlustmodul darstellt. Der Zusammenhang dieser Materialkonstanten ist über den Phasenwinkel wie folgt verknüpft:

$$
\frac{E^{\prime \prime}}{E^{\prime}}=\frac{\sin \delta \cdot \frac{\sigma}{\epsilon}}{\cos \delta \cdot \frac{\sigma}{\epsilon}}=\tan \delta
$$

In Abbildung 1.4 ist dieser Prozess schematisch dargestellt. Man kann die dynamische Kraft in rot und die Antwort des Materials in schwarz erkennen, wobei das Material mit einer Verzögerung $\delta$ der Kraft folgt.

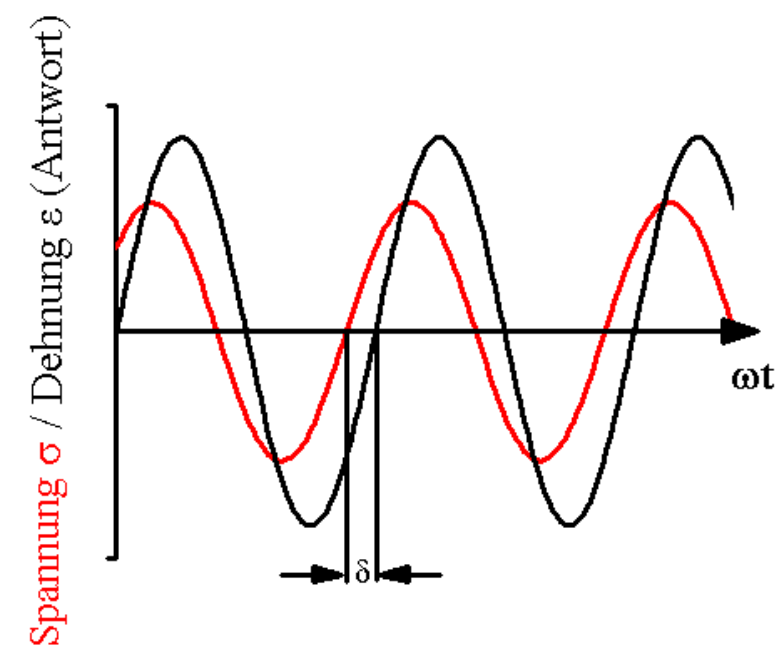

Abbildung 1.4: Bestimmung des Phasenwinkels

Die Materialkonstanten E' und E" kann man über den Phasenwinkel nun wie folgt berechnen:

$$
E^{\prime}=\cos \delta \cdot \frac{\sigma}{\epsilon} \quad \text { und } \quad E^{\prime \prime}=\sin \delta \cdot \frac{\sigma}{\epsilon}
$$




\subsubsection{Spannungs-Dehnungskurven}

Eine weitere Methode, mit Hilfe einer DMA Relaxationsprozesse zu untersuchen, ist, Spannungs-Dehnungskurven aufzunehmen. ${ }^{[19]}$

Hierzu wird nicht, wie im Kapitel 1.3.1.1 beschrieben, mit einer statischen und einer dynamischen Kraft gemessen, sondern bei einer konstanten Dehnungs- oder Spannungsrate. In Abbildung 1.5 ist eine Spannungs-Dehnungskurve, aufgenommen bei einer konstanten Dehnungsrate, aufgetragen. Dabei wird die zu erreichende Dehnung vorgegeben und die Spannung, um diese zu erreichen, gemessen. Man unterscheidet man folgende Bereiche, die das Material durchläuft:

Am Anfang ist die benötigte Spannung proportional zur Dehnungsrate (linearer, elastischer Bereich). Danach wird ein Maximum in der Kurve erreicht (Yielding Point), der im nichtlinearen Bereich der Spannungs-Dehnungskurve liegt. Hier erweicht das Material und kann nun mit viskosem Fließen auf die Spannung reagieren. Die gemessene Spannung sinkt dementsprechend wieder ab, da weniger Kraft benötigt wird, um die vorgegebene Dehnung zu erreichen. In diesem Bereich treten auch die ersten Einschnürungen im Material auf (siehe Abb. 1.6), so dass es notwendig ist, die veränderte Probengeometrie zu berücksichtigen. Die Messung endet mit dem Bruch oder dem Riss des Probenkörpers.

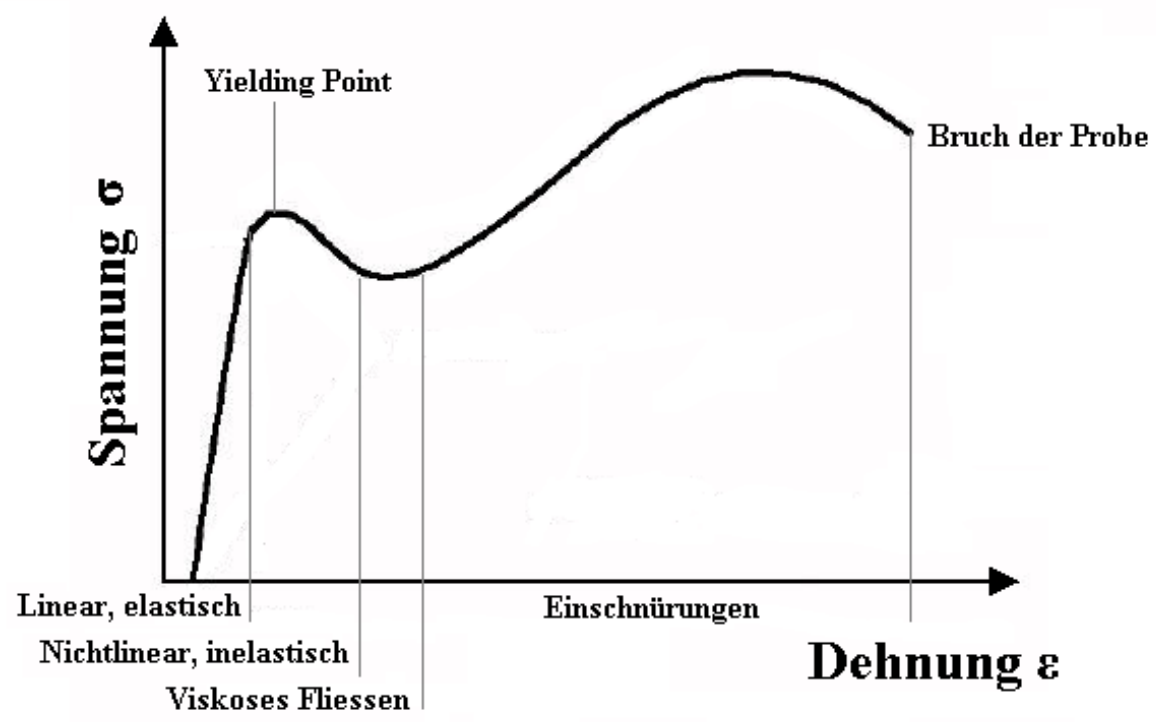

Abbildung 1.5: Spannungs-Dehnungskurve bei konstanter Dehnungsrate 


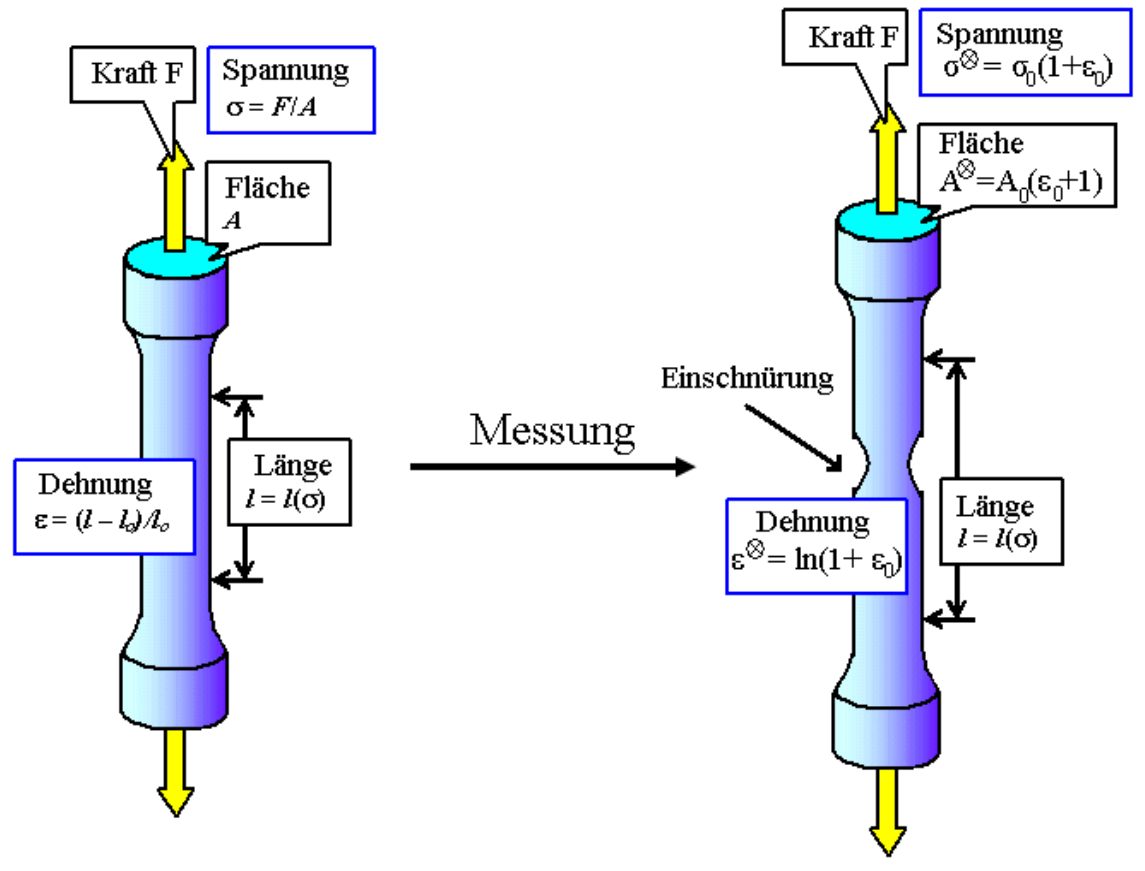

Abbildung 1.6: Veränderung des Probenquerschnitts bei Spannungs-Dehnungskurven modifiziert nach Föll ${ }^{[20]}$

Im linearen Anfangsbereich kann über die Steigung der Elastizitätsmodul bestimmt werden:

$$
E=\frac{\Delta \sigma}{\Delta \epsilon}
$$

Im nichtlinearen Bereich ist dies nicht mehr möglich. Um dennoch die wahre Spannung und Dehnung anzugeben, muss wie in Abbildung 1.6 dargestellt die Einschnürung auf die neue Querschnittsfläche $\mathrm{A}^{\oplus}$ beachtet werden: ${ }^{[20]}$

$$
A^{\oplus}=A_{0} \cdot\left(\epsilon_{0}+1\right)^{-1}
$$

Damit ändert sich in diesem Bereich die Spannung zur wahren Spannung $\sigma^{\oplus}$ und die Dehnung zur wahren Dehnung $\epsilon^{\oplus}$ nach

$$
\begin{gathered}
\sigma^{\oplus}=\sigma_{0} \cdot\left(1+\epsilon_{0}\right) \\
\epsilon^{\oplus}=\ln \left(1+\epsilon_{0}\right)
\end{gathered}
$$


Bei Messungen mit konstanter Spannungsrate wird die angelegte Kraft kontinuierlich erhöht und die erreichte Dehnung gemessen. Bei dieser Art der Spannungs-Dehnungskurven wird der Yielding Point nicht über ein Maximum in der Kurve bestimmt (da die Spannung niemals zurückgehen kann), sondern über eine Tangentenmethode. Sowohl der lineare Anfangsbereich, als auch der Bereich des viskosen Fließens werden durch Geraden verlängert, deren Schnittpunkt dem Yielding Point entspricht. Die Gleichungen 1.9 - 1.12 sind für diese Art der Spannungs-Dehnungskurven ebenfalls gültig. Vorteile bietet diese Messmethode, da es im Bereich des viskosen Fließens keine Einschränkungen über Regelparameter des Messgerätes, wie z. B. die Bestimmung einer fiktiven Dehnungsrate durch instantane Umrechnung der Spannung in die Dehnungsrate gibt. In Abbildung 1.7 ist beispielhaft eine Spannungs-Dehnungskurve mit konstanter Spannungsänderung zu sehen. Es sind ebenfalls der lineare, elastische sowie der nichtlineare Bereich, das viskose Fließen und der Bruch der Probe zu erkennen.

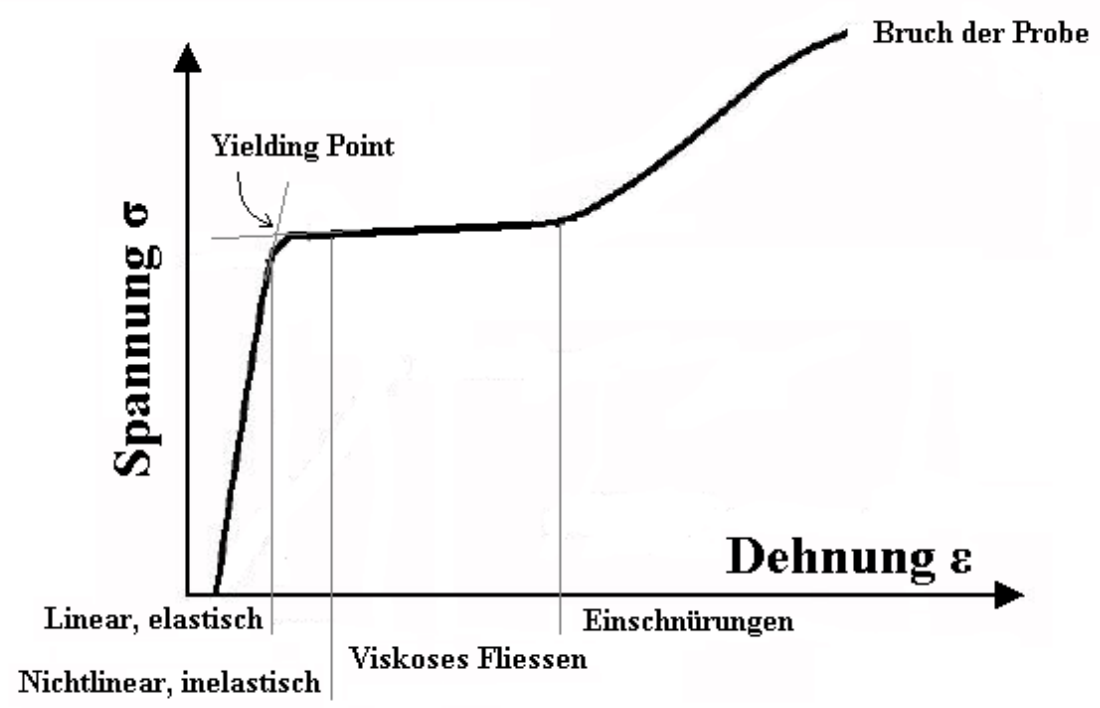

Abbildung 1.7: Spannungs-Dehnungskurve mit konstanter Krafterhöhung 


\subsubsection{Creep/Recoverymessungen}

Eine weitere wichtige Analysemethode für Relaxationsvorgänge sind Creep/Recoverymessungen. Hierbei wird im Gegensatz zu Messungen mit einer dynamischen Kraft keine periodische Änderung der zweiten Kraft verwendet, sondern diese in einem Kraftsprung verändert. Somit kann kein Phasenwinkel bestimmt, jedoch das Dämpfungsverhalten einer Probe sehr gut beobachtet werden. Bei dieser Methode wird nicht nur eine Relaxationszeit mit einer Frequenz abgefragt, sondern breitbandig die ablaufenden Relaxationsvorgänge. Zudem kann das Fließverhalten der Probe (Rheologie) bei verschiedenen Kräften und Temperaturen untersucht werden.
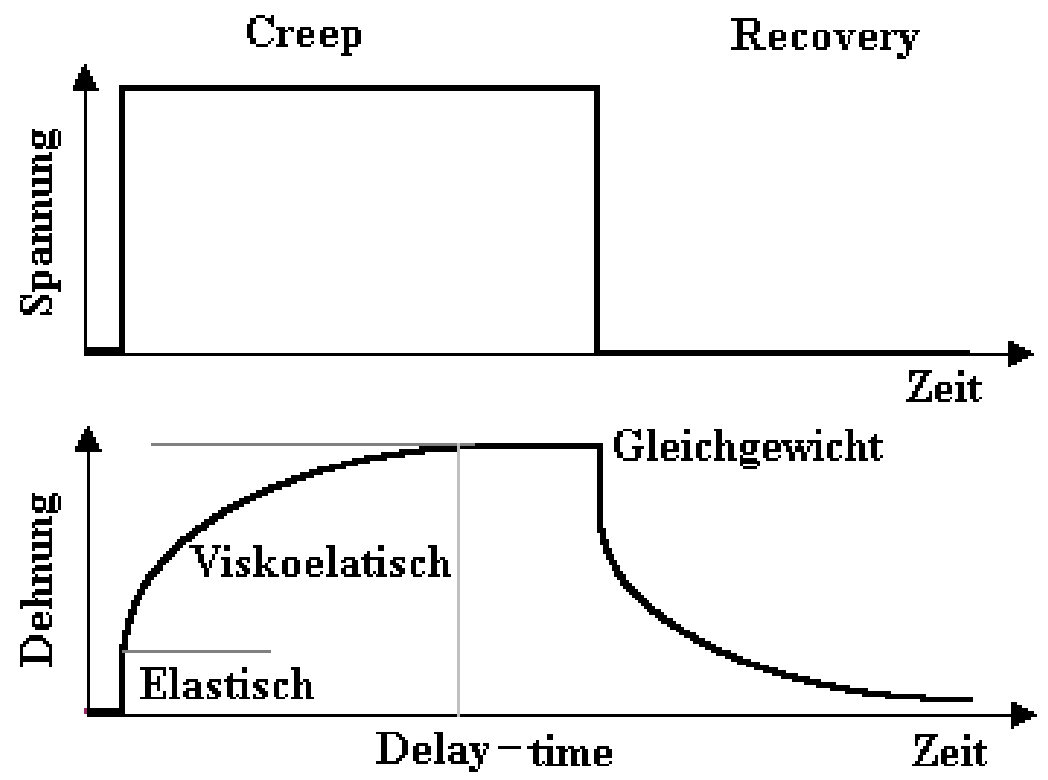

Abbildung 1.8: Creep/Recoverymessung zur Bestimmung der Delay-time

Abbildung 1.8 zeigt eine Creep/Recoverymessung. Im oberen Teil des Bildes sind die Kraftsprünge zu erkennen, die die Messung in die Abschnitte Creep (Kraft wird erhöht) und Recovery (Kraft springt zurück aufs untere Niveau) einteilen. Das Materialverhalten ist in dem unteren Bildteil dargestellt. Zuerst ist eine schnelle, lineare, elastische Materialantwort zu sehen. Auch diese ist proportional zur Spannung, so dass man die Elastizitätskonstante in diesem Bereich wiederum nach Gleichung 1.9 bestimmen kann. Folgend ist ein viskoelastisches Nachkriechen zu beobachten, das durch die Dämpfung der Probe über Verlustprozesse wie Reibung verursacht wird. Nachdem die Spannung abgebaut wurde, wird ein neuer Gleichgewichtszustand erreicht. Die spezifische Zeit bis 
zum Erreichen dieses neuen Zustandes wird als Delay-time definiert, welche proportional zur Dämpfung und somit zum Verlust ist und direkt mit dem Verlustmodul E" verglichen werden kann, welches über dynamische Messungen (siehe Kapitel 1.3.1.1) bestimmt wird. Sobald das Material weicher wird und dementsprechend auch der Verlustmodul ansteigt, wird auch die Delay-time größer, da das Material eine längere Zeit braucht, um sich in den neuen Zustand „einzuschwingen“. Diese Annahme gilt sowohl für den Creep- als auch für den Recovery- Abschnitt der Messung.

\subsubsection{Weitere wichtige Spektroskopiearten}

Um die in Kapitel 3 dargestellten Ergebnisse diskutieren und in die Theorie einordnen zu können, wird auch auf Messungen vorheriger Arbeiten zurückgegriffen, die mit weiteren spektroskopischen Messmethoden aufgenommen wurden. Diese Methoden sollen in diesem Unterkapitel kurz vorgestellt werden.

\subsubsection{Ultraschalluntersuchungen}

Da metallische Gläser für Ultraschallwellen durchlässig sind, kann über Laufzeitmessungen von Ultraschall-Schwingungen sowohl der Schermodul als auch die Dämpfung des Materials bestimmt werden. ${ }^{[22]}$ Über einen piezoelektrischen Schallwandler wird eine Ultraschall-Welle erzeugt, die durch die Probe läuft und an deren Enden reflektiert wird. Die so hin und her reflektierte Schallwelle tritt teilweise wieder in den Schallwandler, worauf ein elektrisches Signal proportional zur Schwingungsamplitude entsteht. Bei genauer Kenntnis der Probenlänge 1 und der Dichte $\rho$ kann aus der Laufzeit einer Scherwelle der Schermodul G berechnet werden, welcher mit dem Elastizitätsmodul E verglichen werden kann. ${ }^{[21,22]}$

\subsubsection{Atomic force acoustic microscopy (AFAM)}

Das AFAM ist ein Ultraschallkraftmikroskop, welches ebenfalls der Bestimmung von elastischen Eigenschaften der Probe dient. Es wird eine Messspitze lokal auf die Probenoberfläche gedrückt, wobei die erreichte Spitzenverbiegung von den lokalen elastischen Eigenschaften der Probe abhängig ist. Während der Messung wird zusätzlich eine Ultraschallwelle in die Probe eingekoppelt. Durch die Aufnahme der Kontaktresonanzen des 
Systems zwischen der Probe und der Messspitze kann die Elastizität bestimmt werden. Des Weiteren ist die Kontaktsteifigkeit von Spitze und Probe auch vom Indentationsmodul M der Probenoberfläche abhängig, welches proportional zum Elastizitätsmodul E ist (siehe Gleichung 1.13 mit $\nu$ als Poissonzahl). Somit kann man eine Kartierung des Probenmaterials nicht nur hinsichtlich der Topographie, sondern auch des Elastizitätsmoduls erreichen. ${ }^{[23-25]}$

$$
M=\frac{E}{1-\nu^{2}}
$$

Durch die hohe Ortsauflösung können kleinste Störungen im Material oder auch dünne Schichten auf der Oberfläche der Probe untersucht werden, die mit makroskopischen Elastizitätsmessungen nicht mehr zugänglich sind. Es ist somit möglich, die Ergebnisse aus DMA-Messungen mit lokalen Veränderungen im Elastizitätsverhalten der Probe zu vergleichen.

\subsubsection{Doppelpaddel-Oszillator (DPO)}

Der Doppelpaddel-Oszillator (DPO) ist ein Oszillator, der sich durch seine spezielle Form besonders dazu eignet, dünne Schichten mechanisch zu vermessen. Es findet eine Anregung in einer seiner Eigenmoden statt, so dass seine Dämpfung z. B. durch Vermessung der Frequenzbreite der Resonanzkurve bestimmt werden kann. Dadurch wird eine sehr präzise Güte der mechanischen Analyse von dünnen Schichten ermöglicht. Dabei ist der Verlustmodul G" (Schermodul) proportional zur bestimmten Güte $\Delta \mathrm{Q}^{-1}$ und somit direkt mit dem Verlustmodul E" aus DMA-Messungen vergleichbar. ${ }^{[26,27]}$ Wie bei der AFAM-Methode besteht die Möglichkeit, reduzierte Probengeometrien hinsichtlich der elastischen Eigenschaften mit Messungen von bulk-Material zu vergleichen. 


\subsection{Relaxationen im Glaszustand}

Nachdem in dem Kapitel 1.3 eine Einführung in die Möglichkeit der mechanischen Untersuchungen von amorphen Festkörpern gegeben wurde, wird in diesem Kapitel ein Überblick über die Relaxationsvorgänge gegeben. Dabei wird gezeigt, dass einige Prozesse allgemein gültig sind und versucht, diesen mikroskopische Prozesse zuzuordnen. Außerdem werden die einzelnen Prozesse über eine Energielandschaft verknüpft (Kapitel 1.4.1), welche in einer Spannungsabhängigkeit beschrieben wird (Kapitel 1.4.3). Es werden dabei sowohl frequenz- als auch temperaturabhängige Prozesse besprochen und deren Abhängigkeit zueinander diskutiert.

Unter Relaxationen versteht man Prozesse, mit denen das Material auf äußere Einflüsse wie beispielsweise externe Spannungen reagiert und diese abbaut. Zu untersuchen sind die Prozesse z. B. über mechanische Spektroskopie (siehe Kapitel 1.3). Dabei können die Relaxationen über verschiedene Dämpfungsverhalten sichtbar gemacht werden.

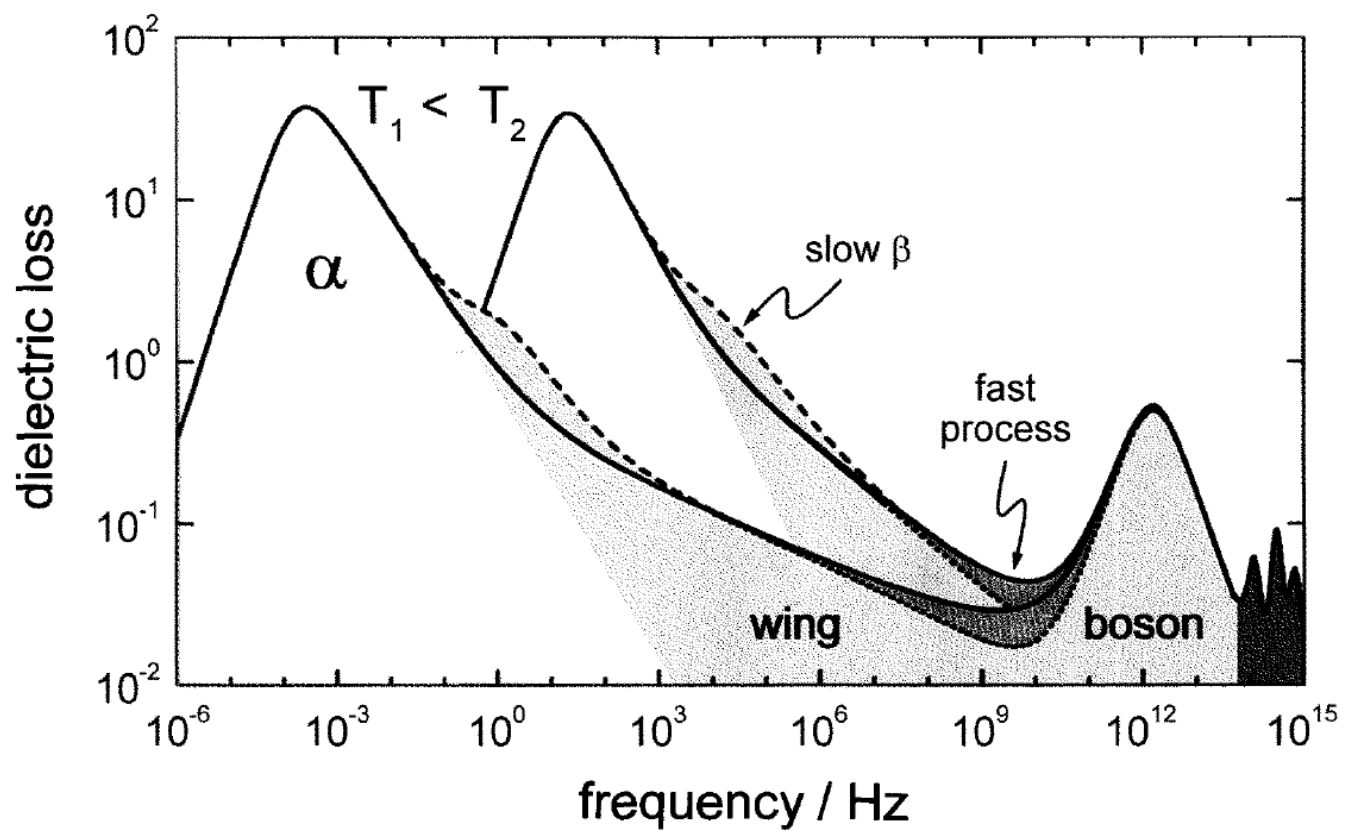

Abbildung 1.9: Lunkenheimer-Loidl-Plot: Schematische Darstellung des durch Relaxationen hervorgerufenen Verlustspektrums eines amorphen Materials ${ }^{[32]}$

Abbildung 1.9 zeigt ein charakteristisches Relaxationsspektrum eines amorphen Systems, welches mittels dielektrischer Spektroskopie untersucht wurde. ${ }^{[32]}$ Es ist der dielektrische Verlust bei zwei verschiedenen Temperaturen als Funktion der Anregungsfrequenz aufgetragen. Da davon ausgegangen wird, dass einige Prozesse aufeinander aufbauen, ist 
das Spektrum von rechts nach links zu beschreiben. Zu erkennen sind die verschiedenen Relaxationen von Molekülschwingungen, welche im Infrarotbereich oberhalb von $10^{14} \mathrm{~Hz}$ zu finden sind. ${ }^{[33]}$ Darunter bis etwa $10^{10} \mathrm{~Hz}$ sind Gitterschwingungen dominierend, welche kooperative Schwingungen darstellen und als Boson-Peak bezeichnet werden. ${ }^{[34,35]}$ Hierauf folgend ist ein Minimum und ein annähernd konstanter Bereich zu beobachten (Nearly Constant Loss (NCL) oder fast $\beta$ - Prozess), welcher durch Cage-Ratteling beschrieben wird. Hierbei können sich Teilchen in einem festen Käfig aus Nachbarteilchen bewegen, diesen jedoch nicht verlassen. Sowohl der Boson-Peak als auch der NCL-Bereich werden sehr gut über die Modenkopplungstheorie beschrieben. ${ }^{[36]}$ Bei tieferen Frequenzen ist ein erneutes Ansteigen im Verlustspektrum zu beobachten, welches als Wing, slow $\beta$ - Prozess oder Johari-Goldstein-Prozess bezeichnet wird. Bei einigen Polymeren konnte in diesem Bereich sogar ein separater Peak beobachtet werden. Meistens wird der Wing jedoch durch die angrenzende $\alpha$ - Relaxation (viskoses Fließen), welche durch das große Maximum zu erkennen ist, überlagert. Dies ist jedoch stark von der Temperatur und der Frequenz abhängig. ${ }^{[37-40]}$

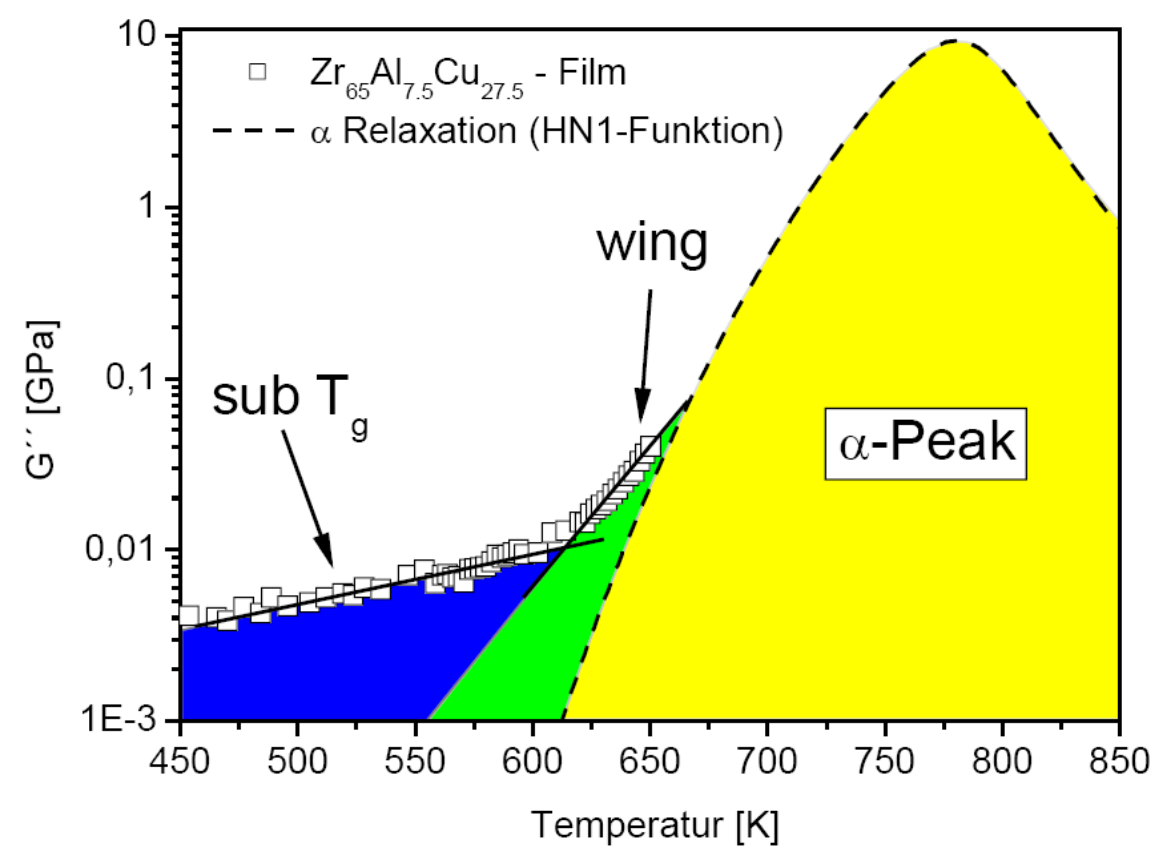

Abbildung 1.10: Slow $\beta$ - Relaxation in einem starken metallischen Glasbildner ${ }^{[13]}$

Die mechanische Spektroskopie an metallischen Gläsern wird, wegen der Abschirmung durch das Elektronensystem, im Gegensatz zu den dieelektrischen Messungen an Po- 
lymeren bei einer festen Spektroskopiefrequenz durchgeführt. Dabei werden die verschiedenen Relaxationen über eine konstante Temperaturerhöhung angeregt. Das gesamte Spektrum liest sich deshalb von links nach rechts. ${ }^{[13]}$ Dabei geht man davon aus, dass sich bei verschiedenen Temperaturen zwar die Position des Maximums der Verteilungsfunktion für die Relaxationszeiten verschiebt, dessen Form jedoch erhalten bleibt. So erhält man mit beiden Prozessführungen die gleiche Information. Dass diese unterschiedliche Herangehensweise zu denselben Resultaten führt, drückt sich im Zeit-Temperatur-Superpositionsprinzip aus, das auch in metallischen Gläsern mit Erfolg bestätigt wurde. ${ }^{[41]}$

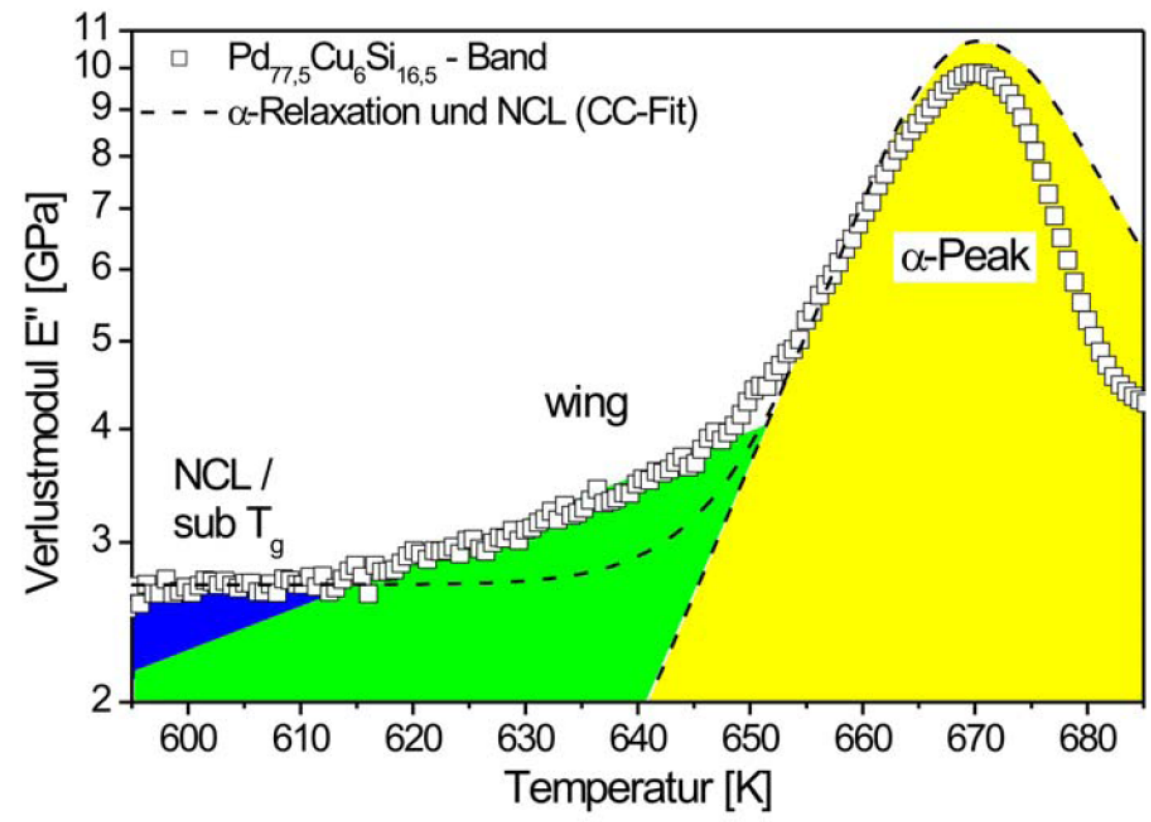

Abbildung 1.11: Slow $\beta$ - Relaxation in einem fragilen metallischen Glasbildner ${ }^{[14]}$

Rösner et al. konnten zeigen, dass auch in metallischen Gläsern dieselben Relaxationen zu finden sind. In dem starken Glasbildner $\mathrm{Zr}_{65} \mathrm{Al}_{7,5} \mathrm{Cu}_{27,5}$ konnten durch DPO-Messungen (siehe Kapitel 1.3.2.3) ein Sub- $\mathrm{T}_{G}$-Bereich und ein Wing festgestellt werden (siehe Abb. 1.10). Durch die frühe Kristallisation war es jedoch nicht möglich, in den Bereich der unterkühlten Schmelze zu gelangen. Somit konnte der Übergang von der $\beta$ - zur $\alpha$ - Relaxation nicht vollständig untersucht werden. ${ }^{[13]}$ Den Beweis für die Allgemeingültigkeit dieser Relaxationen in metallischen Gläsern erbrachten Hachenberg et al., da in dem fragilen Glasbildner $\mathrm{Pd}_{77,5} \mathrm{Cu}_{6,0} \mathrm{Si}_{16,5}$ ebenfalls ein Wing und auch der $\alpha$ - Übergang beobachtet wurden. ${ }^{[14,42]}$ In Abbildung 1.11 ist der Verlustmodul des fragilen Glasbildners 
zu sehen. Es ist zu erkennen, dass der Wing weit unterhalb der Glasübergangstemperatur (ca. $645 \mathrm{~K}$ ) bei $610 \mathrm{~K}$ einsetzt und ab $\mathrm{T}_{G}$ vom $\alpha$-Prozess überlagert wird. Der $\alpha$ - Prozess konnte aufgrund der früh einsetzenden Kristallisation ebenfalls nicht weit in den Bereich der unterkühlten Schmelze verfolgt werden. Die gestrichelte Linie gibt den zu erwartenden Verlauf des $\alpha$ - Peaks an, so dass die beginnende Kristallisation gut zu erkennen ist.

Der Übergang vom dominierenden Bereich der $\beta$ - Relaxation bis hin zur $\alpha$ - Relaxation (in den Spannungs-Dehnungskurven diskutiert als der Übergang vom linearen zum nichtlinearen Bereich, siehe Kapitel 1.3.1.2) ist bis heute noch nicht vollständig verstanden. Die makroskopischen Eigenschaften des Wings und des $\alpha$ - Peaks werden in der Literatur mikroskopisch als verschiedene atomare Anregungen interpretiert. Wie am Anfang dieses Kapitels vorgestellt, werden im NCL bzw. im Sub- $\mathrm{T}_{G}$-Bereich einzelne Atome angeregt, welche sich in einem Käfig der Nachbaratome bewegen, diesen jedoch nicht verlassen können. Bei höheren Temperaturen wird dieses Bild auf Atomketten mit bis zu 20 Atomen erweitert, welche sich in einem quadrupolartigen Verhalten kooperativ bewegen können. ${ }^{[43]}$

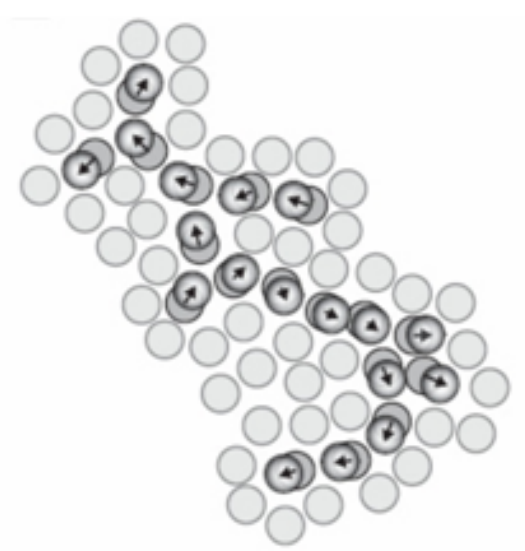

Abbildung 1.12: Kettenartige, quadrupolförmige Anregung der slow $\beta$ - Relaxation ${ }^{[43]}$

In Abbildung 1.12 ist ein solcher Prozess schematisch dargestellt. Man kann erkennen, dass durch „Hineinschieben“ von Atomen in die Kette (siehe Pfeilrichtung) eine Bewegung ausgelöst wird. Dadurch, dass das Atomgerüst in diesem Bereich weit unterhalb der Glasübergangstemperatur sehr starr ist, können sich nur alle beteiligten Atome zusammen bewegen. Zusätzlich entsteht ein großes Spannungsfeld (bezeichnet als Eshelby-Spannungsfeld ${ }^{[44]}$, welches das „Zurückspringen“ der Atomkette begüns- 
tigt. Somit handelt es sich bei diesem Prozess um eine reversible, elastische Anregung (schematisch als 2D-Grenzfall in Abb. 1.13 dargestellt).

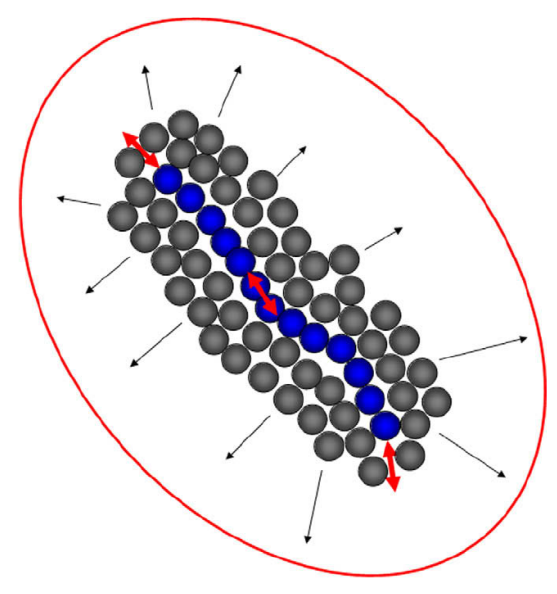

Abbildung 1.13: Schematische Darstellung eines kollektiven, kettenartigen $\beta$ - Prozesses mit zugehörigem Spannungsfeld (rote Linie) ${ }^{[45]}$

Bei DPO-Untersuchungen an dünnen Schichten (siehe Kapitel 1.3.2.3) konnte gezeigt werden, dass der Wing und somit die zugehörige $\beta$ - Relaxation ab einer Schichtdicke von $25 \mathrm{~nm}$ verschwindet. ${ }^{[45]}$ Dabei ist der Verlustmodul als Differenz der Güte $\mathrm{Q}^{-1}$ angegeben (siehe Abb. 1.14)

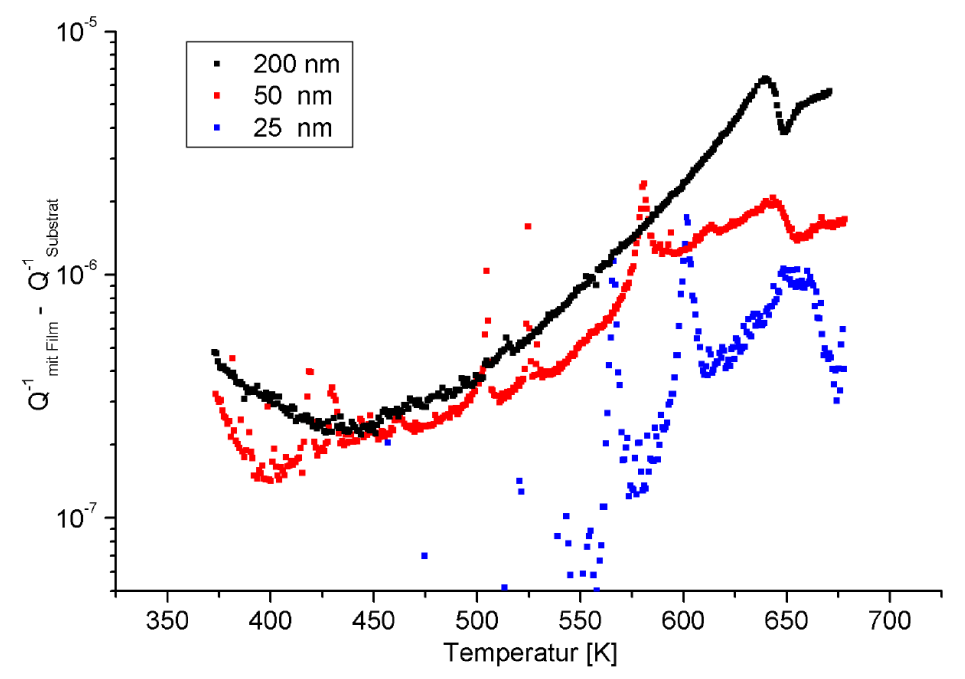

Abbildung 1.14: Verschwinden des durch $\beta$ - Relaxation induzierten Verlustprozesses ab einer Schichtdicke von $25 \mathrm{~nm}^{[45]}$

Bei einer zu kleinen Schichtdicke annihiliert das Spannungsfeld an die Oberfläche und verhindert somit das Zurückspringen der Atomkette. 
Werden in einem bulkartigen Material diese Prozesse jedoch verstärkt, z. B. durch Anregung über Erhöhung der Temperatur, so können die Spannungsfelder überlappen und es bilden sich lokale, sehr stark unter Spannung stehende Bereiche.

Eine der ersten Versuche, die Mechanismen für Deformationen und Fließverhalten von metallischen Gläsern und Flüssigkeiten zu beschreiben, geschah 1979 von Argon. ${ }^{[51]}$ Es wurden lokale Zonen von etwa 10 Atomen vorhergesagt, die über plastische Umorientierung Spannungen abbauen (Scher-Transformations-Zonen - STZs). Diese Bereiche sind in ein elastisches Medium eingebettet und besitzen ein wie von Eshelby vorgeschlagenes Spannungsfeld. ${ }^{[44,51-55]}$ Weiterhin wurde vorgeschlagen, dass die isolierten STZ-Bereiche zu einem reversiblen Speichern von Energie in dieser Matrix führt, wenn die umorientierten STZ-Regionen eine „Erinnerung“ an ihre Originalkonfiguration besitzen.

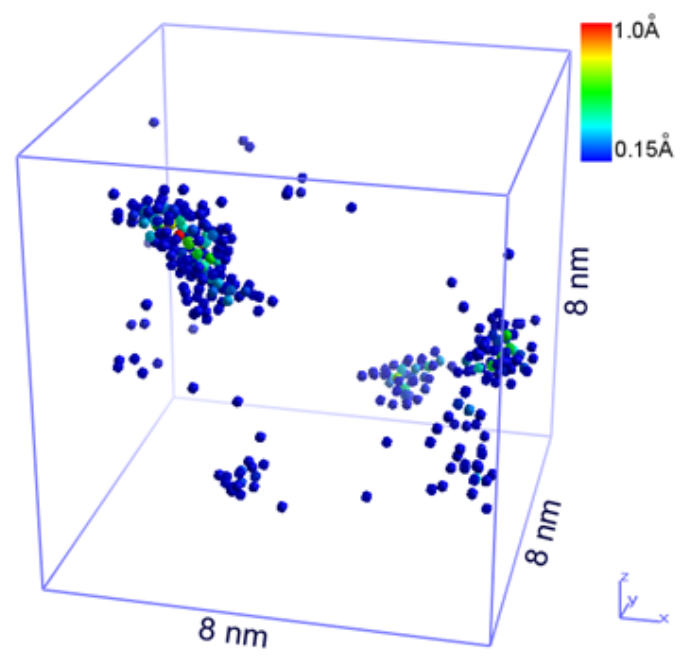

Abbildung 1.15: Durch Scherung angeregte lokalen STZs. Die blauen Cluster geben die Verschiebungen der Atome nach der Scherung wieder. ${ }^{[46]}$

In molekulardynamischen Simulationen wurden an würfelförmigen, amorphen Körpern Scherungen durchgeführt und die Änderung in den Atompositionen verfolgt. Es konnte gezeigt werden, dass sich lokale Bereiche bilden, in denen sich die Atome wie flüssig verhalten und eine neue, nichtreversible Konfiguration annehmen. Dieses Verhalten wird mit der $\alpha$ - Relaxation in Verbindung gebracht und ist in Abbildung 1.15 dargestellt. ${ }^{[46]}$ Die Farbkodierung (blaue Cluster) gibt die Verschiebung der Atome nach der Scherung wieder. Zu erkennen sind die lokalen Bereiche (STZs), in denen sich die starke Spannung plastisch abbauen konnte. 


\subsubsection{Potenzielle Energielandschaft (PEL)}

In diesem Kapitel wird die Idee einer potentiellen Energielandschaft (PEL) nach Stillinger und Weber zur Beschreibung von $\beta$ - und $\alpha$ - Prozessen vorgestellt. Sie beschreibt quantitativ die mechanischen Eigenschaften des Glases, wie auch die Viskosität der Schmelze, wobei man zwei Anregungsarten unterscheidet. Zum einen wird das System über den $\alpha$ - Prozess durch viskoses Fließen in eine neue Konfiguration mit geänderter potentieller Energie überführt und zum anderen findet über lokale reversible Konfigurationsänderungen der Spannungsabbau statt. ${ }^{[47-50]}$

Nachdem Johari und Goldstein zwei grundlegende Relaxationsprozesse zum Spannungsabbau bei Gläsern und Flüssigkeiten vorschlugen, konnten erstmals die vorhergesagten spannungsabhängigen STZ-Prozesse zugeordnet werden. ${ }^{[56]}$ Es wurden zwei kinetische Prozesse empfohlen, ein schneller Prozess, der dem in Kapitel 1.4 genannten slow $\beta$ - Prozess entspricht und ein langsamer, welcher mit dem vorgestellten $\alpha$ - Prozess übereinstimmt. Der $\beta$ - Prozess stellt eine sehr lokal initiierte und reversible Relaxation im Gegensatz zum $\alpha$ - Prozess dar, der auf einer größeren Längenskala mit einer irreversiblen Konfigurationsänderung beschrieben wird (siehe Kapitel 1.4). Diese Prozesse sollen in der PEL schematisch über eine Änderung in der potenziellen Energie miteinander in Verbindung gebracht werden.

Zur Abbildung der PEL werden die potentiellen Energien aller n Atome der Probe auf eine Potentialachse übertragen. Diese wird gegen eine generalisierte Koordinatenachse aufgetragen. Somit wird die 3-dimensionale Potentiallandschaft aller Atome auf einen 2D-Plot reduziert. Dies hat den Vorteil, dass die Prozesse anschaulich dargestellt werden können und die Energielandschaft sowohl für die Diskussion der makroskopischen Prozesse als auch für lokale Anregungen verwendet werden kann.

In Abbildung 1.16 ist das Bild der PEL dargestellt. Im oberen Bildteil ist die PEL über die generalisierte Koordinate über mehrere Minima (Metabasins) abgebildet. Das untere Bild zeigt eine Vergrößerung von zwei Metabasins mit einer Feinstruktur (Subbasins) aus der gesamten Potenziallandschaft. Dabei konnten Johnson und Samwer zeigen, dass eine sinusförmige Funktion die potentielle Energiedichte der Metabasins für den $\alpha$ - Prozess gut beschreibt. ${ }^{[47]}$

Die beschriebenen Prozesse werden mit den roten Buchstaben $\beta$ und $\alpha$ bezeichnet. In der Darstellung der PEL nach Debenedetti und Stillinger werden die $\beta$ - Übergänge 


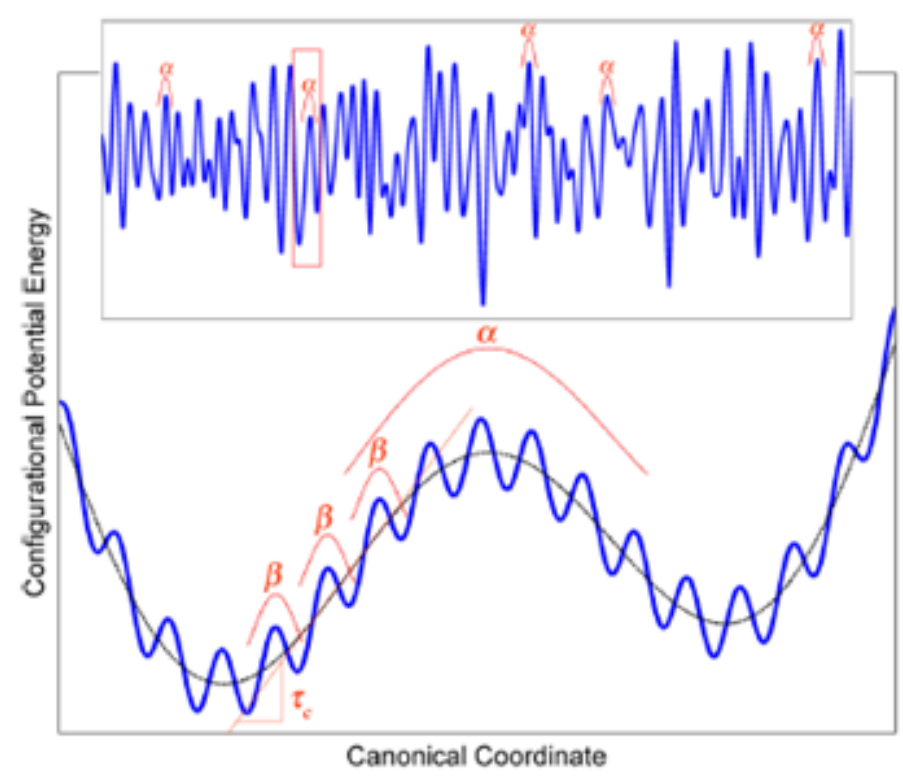

Abbildung 1.16: Relaxationsprozesse im Bild einer potenziellen Energielandschaft ${ }^{[50]}$

als statistische „hopping events“ in der Feinstruktur (Übergang von einem Subbasin zum nächsten) beschrieben, welche als intrabasin hopping bezeichnet werden. Konnten genügend Events aktiviert und somit in der PEL in ein hohes Subbasin angeregt werden, so ist es möglich, über die Barriere des Metabasins zu gelangen und eine neue Konfiguration zu erreichen (z. B. über Temperaturerhöhung). Dieser Übergang wird als interbasin hopping (Erreichen eines neuen Metabasins) bezeichnet und entspricht dem irreversiblen $\alpha$ - Prozess. ${ }^{[10]}$

\subsubsection{Alterungsprozesse}

Der Vergleich der mechanischen Eigenschaften von metallischen Gläsern vor und nach thermischer Vorbehandlung lässt Rückschlüsse auf Alterungsprozesse zu. Dabei werden die Ergebnisse mit Hilfe der PEL versucht zu interpretieren. Unter einer Alterung im Sinne der PEL versteht man, wenn die Probe entweder durch $\beta$ - Relaxation innerhalb eines Metabasins in der Energielandschaft in Richtung Minimum relaxiert oder durch $\alpha$ - Prozesse günstigere Konfigurationen eingenommen werden. Hierdurch verschiebt sich $\mathrm{T}_{G}$ in Richtung höherer Temperaturen. ${ }^{[57-60]}$

Bei der Untersuchung der $\beta$ - Alterung zeigten Hachenberg et al., dass sich wesentliche Veränderungen in den Moduli finden lassen (siehe Abb. 1.17). Dabei wurden Proben mit- 

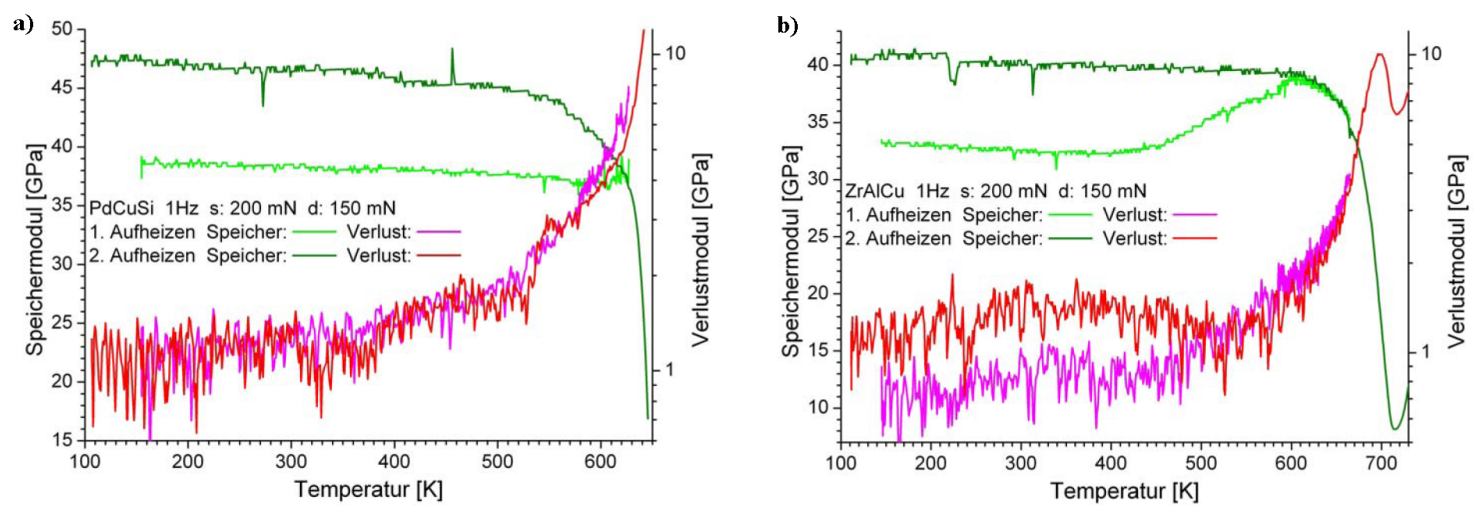

Abbildung 1.17: Effekt der thermischen und mechanischen Vorbehandlung auf die Elastizitätsmodule von a) $\mathrm{PdCuSi}$ und b) $\mathrm{ZrAlCu}^{[14]}$

tels mechanischer Spektroskopie bei konstanter Heizrate untersucht. Die Proben wurden bis knapp unterhalb der Glasübergangstemperatur geheizt, wieder abgekühlt und erneut erwärmt. Hierbei war zu beobachten, dass der Speichermodul sowohl beim fragilen Glas PdCuSi als auch beim starken Glasbildner $\mathrm{ZrAlCu}$ zu höheren Werten verschoben ist. Im Verlustmodul waren keine signifikanten Unterschiede bestimmbar. ${ }^{[14]}$ Dies lässt darauf schließen, dass das Glas durch die $\beta$ - Relaxationen, die in diesem Temperaturbereich dominierend sind, in eine stabilere Konfiguration innerhalb eines Metabasins relaxieren konnte, so dass das Material steifer wurde und einen höheren Speichermodul erreicht. Eine genauere Untersuchung der $\beta$-Alterung wurde bei Versuchen durchgeführt, bei denen der Speichermodul systematisch nach einer Temperaturauslagerung bestimmt wurde. ${ }^{[61]}$ In Abbildung 1.18 sind die Ergebnisse hierzu dargestellt. Der Schermodul (über Ultraschallmessungen bestimmt, siehe Kapitel 1.3.2.1) wurde gegenüber der Auslagerungstemperatur aufgetragen. Bei den Versuchen wurde die Probe in mehreren Zyklen bis zur Glasübergangstemperatur ausgelagert. Die genaue Versuchsbeschreibung ist in ${ }^{[61]}$ dargestellt. Im ersten Zyklus ist ein Ansteigen des Moduls bis hin $\mathrm{zu} \mathrm{T}_{G} \mathrm{zu}$ beobachten, gefolgt von einem Abfall, welcher über $\alpha$ - Relaxationen zu diskutieren ist. Beim zweiten bis vierten Zyklus (derselben Probe) ist ein höheres Schermodul am Anfang zu beobachten. Dies entspricht den Ergebnissen, die in Abbildung 1.17 diskutiert wurden. Jedoch ist kein kontinuierlicher Anstieg mehr zu erkennen. Beim zweiten Zyklus steigt der Modul noch kurz an, fällt danach aber weit unterhalb von $\mathrm{T}_{G}$ wieder ab. Bei den folgenden Zyklen ist nur noch ein Absinken mit höherer Auslagerungstemperatur zu 
erkennen. Diskutiert werden diese Ergebnisse über Alterungs- und Verjüngungsprozesse.

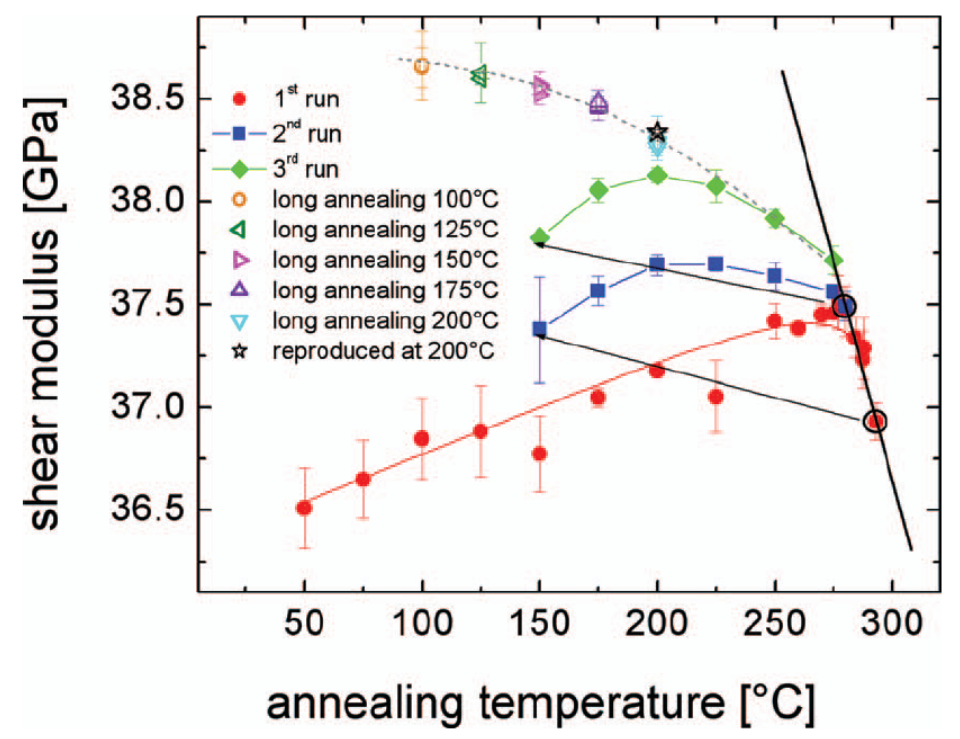

Abbildung 1.18: Alterung durch Auslagerungsexperimente ${ }^{[61]}$

Da die verwendete Probe nach der Herstellung in einem nicht näher bestimmbaren Zustand innerhalb der PEL eingefroren wird, werden bei den Auslagerungen zunächst $\beta$ - Relaxationen angeregt, die dazu führen, dass die Probe in einen tieferen Zustand innerhalb eines Metabasins in der PEL relaxieren kann. Dies führt dazu, dass die Probe härtet und ein höheres Modul bei den Ultraschallmessungen zu bestimmen ist. Beim zweiten und dritten Zyklus war die Alterung fast vollständig abgeschlossen, so dass nur noch eine kleine Erhöhung im Modul zu beobachten ist. Danach dominierten die thermischen Anregungen der $\beta$ - Relaxationen. Es werden wieder höhere Subbasins erreicht (Verjüngung), so dass der Modul sinkt. Beim vierten Zyklus ist nur noch ein kleiner werdendes Schermodul zu erkennen, da keine Alterung mehr stattfindet, sondern nur noch Prozesse, die eine Verjüngung der Probe zur Folge haben. ${ }^{[61]}$ Ein weiteres Anzeichen für die Alterung eines metallischen Glases ist in Abbildung 1.19 dargestellt. Untersucht wurde ein ca. 200 nm dicker Film über die Doppelpaddel-Oszillator-Methode (siehe Kapitel 1.3.2.3). Am Anfang ist das Absinken im komplexen Schermodul und zugehörig das Ansteigen im realen Part von $370 \mathrm{~K}$ bis $420 \mathrm{~K}$ zu erkennen. Dieses Verhalten wird durch Relaxationen in tiefere Subbasins durch das thermische Anlassen erklärt. Darauf folgend findet die thermisch getriebene Verjüngung über $\beta$ - Relaxationen statt, die den bekannten Wing darstellt. Anschließend ist bei ca. $630 \mathrm{~K} \mathrm{~T}_{G}$ zu erkennen 
(Knick in den Modulen) und die Kristallisation bei ca. $640 \mathrm{~K} .{ }^{[27]}$

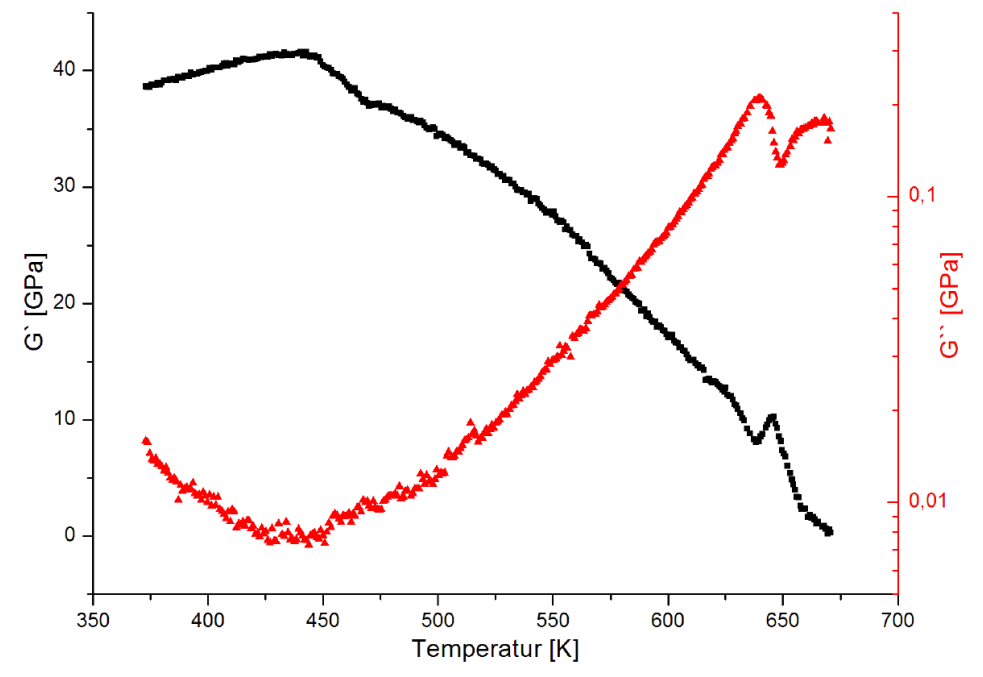

Abbildung 1.19: Alterung der Probe bis ca. $450 \mathrm{~K}$ durch thermisches Anlassen beim Erwärmen ${ }^{[27]}$

\subsubsection{Cooperative Shear Model (CSM)}

Mithilfe von Spannungs-Dehnungskurven von metallischen Gläsern wurde der Übergang vom linearen, elastischen Bereich zum nichtlinearen untersucht. ${ }^{[50]}$ In Abbildung 1.20 sind einige Spannungs-Dehnungskurven vom amorphen $\mathrm{Pd}_{43} \mathrm{Ni}_{10} \mathrm{Cu}_{27} \mathrm{P}_{20}$ abgebildet. Es wurde das Antwortverhalten der Proben $20 \mathrm{~K}$ unterhalb der Glasübergangstemperatur bei einer Dehnungsrate von $10^{-4} \mathrm{~s}^{-1}$ beobachtet, wobei sechs verschiedene Maximaldehnungen (von a) 0,0102 b) 0,0335 c) 0,0655 d) 0,0952 e) 0,1185 bis f) 0,2110) erreicht wurden. $\mathrm{Zu}$ erkennen ist, dass der Übergang vom elastischen Verhalten zum viskosen Fließen über die Erhöhung der externen Spannung erreicht wird. Dabei konnte eine kritische Scherspannung $\sigma_{\text {krit. }}$ von etwa $1000 \mathrm{MPa}$ beobachtet werden, welche für den Übergang in den nichtlinearen Bereich benötigt wird. Der Yielding Point wurde bei einer Dehnung von etwa $7 \%$ bestimmt.

Unter Einbeziehung der Theorie der potentiellen Energielandschaft (siehe Kapitel 1.4.1), zeigen diese Ergebnisse die Möglichkeit, dass die Position in der Energielandschaft über eine äußere Spannung veränderbar ist. Das Entlanglaufen im linearen, elastischen Bereich der Spannungs-Dehnungskurve wird als Möglichkeit des Erreichens höherer Niveaus (höhere Subbasins) interpretiert, so dass beim Erreichen des Yielding Points die 


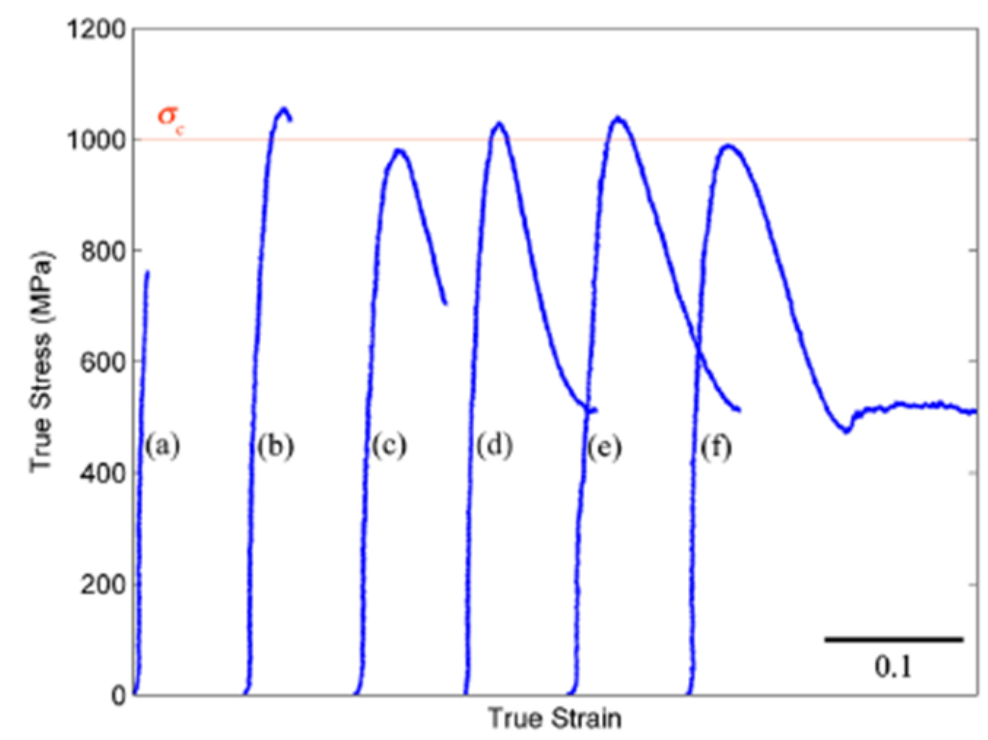

Abbildung 1.20: Spannungs-Dehnungskurven von amorphem $\mathrm{Pd}_{43} \mathrm{Ni}_{10} \mathrm{Cu}_{27} \mathrm{P}_{20}$ bei $548 \mathrm{~K}$ $\left(\mathrm{T}_{G}=570 \mathrm{~K}\right)$ mit einer Dehnungsrate von $10^{-4} \mathrm{~s}^{-1}$. Sechs verschiedene Maximaldehnungen (a-f) wurden erreicht. ${ }^{[50]}$

Überquerung der Barriere zwischen zwei Metabasins möglich wird. Der Übergang vom elastischen zum nichtlinearen, viskosen Bereich bedeutet, dass ein $\alpha$ - Übergang erreicht und somit eine neue, nicht reversible Konfiguration eingenommen wurde.

Da die Temperatur konstant gehalten wurde, ist eine thermische Anregung, wie sie in den Kapiteln 1.4 und 1.4.2 beschrieben wird, nicht möglich. W.L. Johnson und K. Samwer schlagen vor, dass die Barrierenhöhe über die äußere Spannung erniedrigt wird. Erst dadurch wird es möglich, dass die angeregte Probe in höhere Subbasins in der Energielandschaft gelangt, bis zur Möglichkeit, in weitere Metabasins zu wechseln. Sobald dies geschieht, fängt die Probe an, sich plastisch zu verformen, da STZs entstehen, welche die Spannung lokal plastisch abbauen können.

Das Modell der Barrierenerniedrigung ist in Abbildung 1.21 dargestellt. Es wurde ein Frenkelansatz gewählt ${ }^{[63]}$ und auf das Bild der Energielandschaft angewendet. Dazu wurde die potentielle Energie in Abhängigkeit der Dehnung des Materials aufgetragen. Die verschiedenen Kurven stellen dabei die Barrierenhöhe innerhalb der PEL dar, die über eine äußere Spannung $\tau$ erniedrigt wird. Sobald diese der kritischen Spannung $\tau_{c}$ entspricht, verschwindet die Barriere vollständig und ein Übergang in das nächste Metabasin wird spontan möglich. ${ }^{[47]}$ 


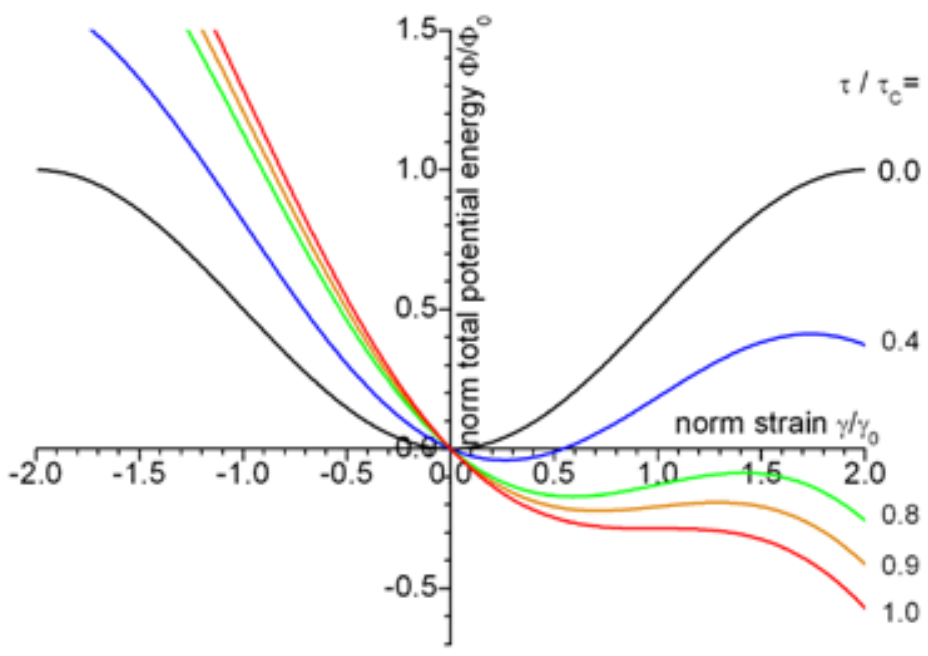

Abbildung 1.21: Frenkelansatz zur Erniedrigung der Barrierenhöhe zwischen zwei Metabasins innerhalb der PEL ${ }^{[47]}$

Unter der Annahme dieses Models sollte es möglich sein, den Zustand innerhalb der PEL nicht nur über eine Veränderung der Temperatur zu erreichen, sondern auch mithilfe einer externen Spannung (wie in Abb. 1.20 beschrieben). Diese Hypothese ist bisher noch nicht abschließend diskutiert, da experimentelle Daten zum Zusammenhang zwischen Temperaturanregung und Absenken der PEL über eine äußere Spannung fehlen. Es besteht jedoch die Möglichkeit, über die Bestimmung der Dämpfung des Materials Rückschlüsse auf den Zustand der PEL zu ziehen. Beim Erreichen höherer Niveaus in der Substruktur innerhalb der PEL nimmt die Dämpfung bis zum $\alpha$ - Übergang zu (Erweichen durch angeregte $\beta$ - Relaxation). Des Weiteren sollte die Glasübergangstemperatur abhängig von der externen Spannung sein, da sie den Punkt des möglichen Übergangs in neue Metabasins darstellt. Über die Erniedrigung der Barrierenhöhe sollte sich dieser Punkt in der Temperatur verschieben lassen.

\subsubsection{Längenskalen von mikroskopischen Prozessen - Aktivierungsvolumen}

Der makroskopische Übergang von einem amorphen Festkörper in die unterkühlte Schmelze wird über mikroskopische Anregungsprozesse beschrieben, die mit verschiedenen lokalen Heterogenitäten erklärt werden. ${ }^{[14,62,64,65]}$ Diese besitzen charakteristische Längenskalen und somit auch definierte Aktivierungsvolumina. ${ }^{[27,66,67]}$ In diesem Kapitel 
soll ein Überblick der Längenskalen, welche für die einzelnen Relaxationsprozesse wichtig sind, gegeben und den Prozessen ein Aktivierungsvolumen zugeordnet werden, welches benötigt wird, um die Prozesse anzuregen.

Allgemein reichen die Längenskalen in metallischen Gläsern vom Abstand nächster Nachbarn (im harten Kugelmodell) bis hin zu Clustern (Scher Transformations-Zonen) von Atomen, welche sich wie Flüssigkeiten verhalten.

Neben dem kleinstmöglichen Abstand (Nachbaratome zueinander) ergibt sich eine charakteristische Längenskala durch die atomare Nahordnung zueinander. Diese relative Atomverteilung kann sowohl aus Streuexperimenten als auch über Computersimulationen bestimmt und miteinander verglichen werden. Dabei zeigen sich charakteristische Anordnungen bis etwa $20 \AA$, wobei eine Anordnung von Ikosaedern (13 - 55 Atome) besonders günstig ist. ${ }^{[8]}$

In der makroskopischen Beobachtung der Gläser bei Temperaturen in der Nähe der Glasübergangstemperatur treten jedoch weitere Heterogenitäten auf, die weiter reichende Längenskalen benötigen. Teichler et al. konnten string-artige Ketten als mikroskopische Ursache der schnellen Relaxationsprozesse identifizieren. ${ }^{[68]}$ Dabei bewegen sich Atome kooperativ in einer kettenartigen Bewegung wie in Kapitel 1.4 vorgestellt. Diese Prozesse wurden als teilweise reversibel beobachtet. ${ }^{[69]}$ Bei der Reduzierung der Dimensionen vom bulkartigen zum Schichtmaterial konnte gezeigt werden, dass die string-artigen Prozesse bei einer Schichtdicke von ca. $25 \mathrm{~nm}$ verschwinden, welches über Eshelby-Stress Felder erklärt wird (siehe auch Kapitel 1.4). ${ }^{[45]}$ Dies gibt einen ersten experimentellen Eindruck für die simulierten Längenskalen wieder.

Bei höheren Temperaturen reichen diese Heterogenitäten jedoch für die starke Viskositätsänderung nicht aus. Bereits 1965 wurden in Modellierungen von Gläsern Cooperative rearranging regions (CRR) als entscheidendes Merkmal von G. Adam und J. H. Gibbs vorhergesagt. ${ }^{[70]}$ Als Weiterentwicklung dieser Idee wurden STZs vorgeschlagen, welche kugelförmige Bereiche mit einigen Atomen Durchmesser beschreiben, die über plastische Verformungen Scherspannungen abbauen können (siehe auch Abb. 1.15).[51] W.L. Johnson und K. Samwer schlagen eine Spannungsabhängigkeit von diesem Modell vor (siehe Kapitel 1.4.3), welches als Cooperative Shear-Modell (CSM) bezeichnet wird. ${ }^{[47]}$ Hierbei werden STZs mit einem Aktivierungsvolumen von bis zu 100 Atomen für ein metallisches Glas vorhergesagt, welche in ein Bild einer potentiellen Energielandschaft 
(PEL) eingeordnet werden (siehe Kapitel 1.4.1). ${ }^{[50]}$ In Molekulardynamik-Studien konnte ein STZ-Durchmesser von ca. 1,5 nm bestimmt werden. ${ }^{[46,71,72]}$

Neuere Publikationen von Chen et al. geben einen ersten experimentellen Eindruck des Aktivierungsvolumens von STZs mit einer Größenordnung von 2,5 nm $\mathrm{nm}^{3}-6,5 \mathrm{~nm}^{3}$, abhängig vom Typ des metallischen Glases. ${ }^{[66,67]}$ Dabei kann die Längenskala durch thermisches Relaxieren der Probe um $50 \%$ reduziert werden. ${ }^{[67]}$

Die Einbettung der bestimmten Längenskalen der STZs in das Cooperative Shear Modell ist jedoch noch nicht gelungen.

Experimentell wird das Aktivierungsvolumen über den Zusammenhang der Dehnungsrate $\dot{\epsilon}$ und der benötigten Spannung $\sigma$ berechnet: ${ }^{[66,67,73]}$

$$
\Omega^{*}=k_{B} \cdot T \cdot m=k_{B} \cdot T \cdot\left(\frac{\partial \ln \dot{\epsilon}}{\partial \sigma}\right)_{p, T}
$$

(mit $\mathrm{k}_{B}$ : Boltzmannkonstante, T: absolute Temperatur und p: Druck).

Der Zusammenhang der Dehnungsrate und der Spannung wird als Dehnungsratensensitivität $\mathrm{m}$ bezeichnet:

$$
m=\left(\frac{\partial \ln \sigma}{\partial \ln \dot{\epsilon}}\right)_{p, T}
$$

Um das Aktivierungsvolumen zu berechnen, ist es notwendig, im inelastischen Bereich des Materials zu sein. Wenn die Dehnungsratensensitivität über einen weiten Temperaturbereich konstant ist, befindet sich das Material jedoch im linearen, elastischen Bereich der Spannungs-Dehnungskurven, so dass die Gleichung 1.14 nicht anwendbar ist. 


\subsection{Polymerdynamik}

Polymerstrukturen lassen weit mehr Relaxationen zu als metallische Gläser, da die Annahme eines Systems aus harten Kugeln nicht mehr gerechtfertigt ist und somit mehr innere Freiheitsgrade vorhanden sind. Durch ihren komplexeren Aufbau mit zum Teil weit verzweigten Strukturen können neben dem Erweichen und der vorgelagerten $\beta$ - Relaxation weitere Bewegungen wie Seitengruppen- oder auch Gerüstbewegungen hinzukommen. Ein Beispielspektrum zeigt Abbildung 1.22, bei dem die Polymere PMMA, PEMA, P-n-PMA und P-n-BMA systematisch mittels mechanischer Spektroskopie untersucht wurden. Neben dem $\beta$ - und $\alpha$ - Peak ist bei tiefen Temperaturen ein weiterer, sogenannter $\gamma$ - Peak zu erkennen. Dieser wird durch eine Wellen- oder auch Kurbelwellenbewegung der Gerüstkette hervorgerufen (siehe Kapitel 1.5.1). ${ }^{[18]}$

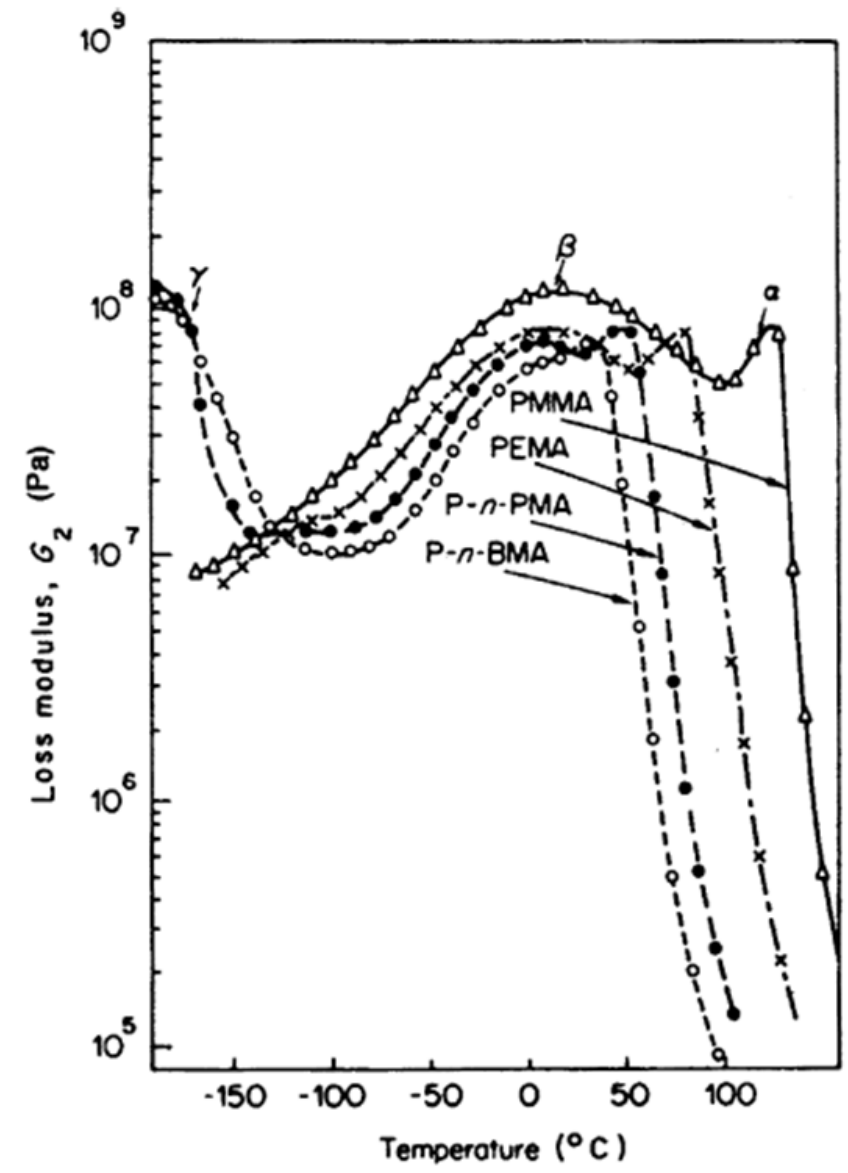

Abbildung 1.22: Temperaturabhängige Relaxationsprozesse von Polymeren ${ }^{[18]}$

Durch die chemischen Eigenschaften der Monomereinheiten können dabei sehr verschiedene Wechselwirkungen in den Polymeren auftreten. Diese Vielfalt in der Mikrostruktur 
spiegelt sich in den makroskopischen, mechanischen Eigenschaften und dem Relaxationsspektrum wieder, da im Prinzip jede Struktureinheit zu weiteren, charakteristischen Relaxationen führen kann.

In den folgenden Unterkapiteln sollen vor allem die Kurbelwellen- und Seitengruppenbewegungen und der Einfluss von chemischen Wechselwirkungen der Monomereinheiten in den Polymeren beschrieben werden.

\subsubsection{Kurbelwellen- und Seitengruppenbewegungen}

Die Relaxationen in Polymergerüsten werden meist als Kurbelwellenbewegungen beschrieben. ${ }^{[74,75]}$ Boyer und Schatzki schlagen dafür eine Drehung der Monomereinheiten in einer linearen Kette mit festen Winkeln zwischen den Molekülen vor, in denen um eine Bindung frei gedreht werden kann. Schatzki geht dabei von acht Monomereinheiten (sieben Bindungen) aus. Die erste und die letzte Bindung ist jedoch fest und nicht drehbar, so dass eine S-förmige Gruppe entsteht, die frei rotieren kann (siehe Abb. 1.23 a). Boyer schlägt dagegen eine Beteiligung von nur sechs Monomereinheiten vor, so dass eine C-förmige Bewegung entsteht (siehe Abb. 1.23 b). Bekannt ist, dass mit einer Anregungsfrequenz von $1 \mathrm{~Hz}$ diese Relaxation für Polyethylen bei ca. $150 \mathrm{~K}$ liegt und als $\gamma$ - Relaxation beschrieben wird. ${ }^{[74]}$

a)

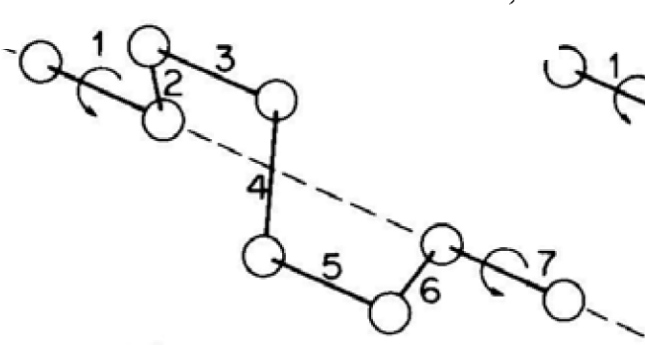

b)

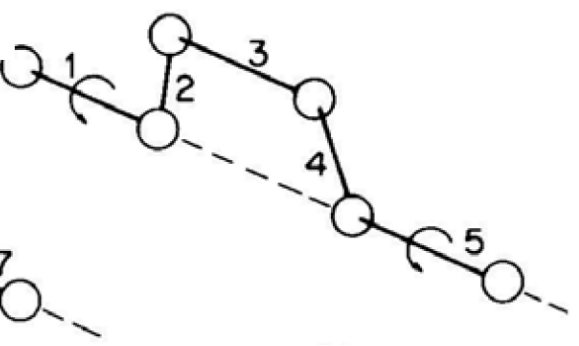

Abbildung 1.23: Kurbelwellenbewegung nach a) Schatzki und b) Boyer ${ }^{[74,75]}$

Bei verzweigten Polymeren können die Seitengruppen ebenfalls zu Relaxationen führen. Abhängig von der Länge der Seitengruppen findet man diese bei höheren Temperaturen als die der Kurbelwellenbewegungen. Bei Polymeren mit Phenylseitengruppen liegt diese Relaxation etwa bei 325 K. ${ }^{[75]}$ Seitengruppenrelaxationen werden meist als $\beta$ - Relaxation beschrieben, welche jedoch nicht mit den $\beta$ - Relaxationen des Wings in metallischen Gläsern (siehe Kapitel 1.4) verwechselt werden darf. In Polymeren wurden aber auch 
vergleichbare Kettenbewegungen (strings) beobachtet, die analog zu den metallischen Gläsern als $\beta$ - Relaxationen bezeichnet werden. ${ }^{[39]}$

\subsubsection{Chemical Confinement}

Bei mechanisch-dynamischen Messungen an Poly(Ethylen-co-Methacrylsäure) (EMAA) wurde das Relaxationsverhalten der Polymere genauer untersucht (die Monomereinheiten sind in Abb. 1.24 zu sehen). Dabei wurden Unterschiede im Temperaturbereich unterhalb der Glasübergangstemperatur im Zusammenhang mit der Herstellung der Polymerketten festgestellt. ${ }^{[14]}$ In Abbildung 1.25 sind Beispielspektren für EMAA dargestellt, bei denen der Zusammenhang zwischen Relaxationsspektren und dem Herstellungsdruck während der Polymersynthese systematisch untersucht wurde.

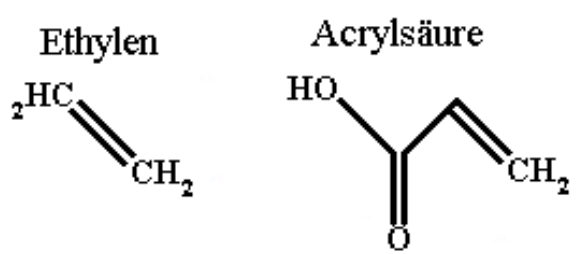

Abbildung 1.24: Verwendete Monomereinheiten bei der Untersuchung des Chemical Confinements

Dabei konnte unter hohem Herstellungsdruck (2000 bar) nur das Erweichen des Polymers festgestellt werden (größer werdender Phasenwinkel bei ca. $300 \mathrm{~K}$ - schwarze Kurve). Bei mittlerem Druck (1800 bar - blaue Kurve) konnte im Phasenwinkel des Relaxationsspektrums sowohl ein $\gamma$ - Peak (ca. $150 \mathrm{~K}$ ) als auch das Erweichen (ca. $300 \mathrm{~K}$ ) beobachtet werden. Bei dem niedrigsten Herstellungsdruck (1300 bar - rote Kurve) wurde im Temperaturbereich zwischen dem $\gamma$ - Peak und dem Erweichen ein weiterer Relaxationspeak beobachtet (ca. 220 K). Es ist jedoch anzumerken, dass bei allen Messungen mit verschieden großen statischen und dynamischen Kräften und verschiedenem MAA-Gehalt gearbeitet wurde und dieser Zusammenhang nicht systematisch untersucht wurde.

Der zusätzliche Peak wurde durch ein Wasserstoffbrücken induziertes Chemical Confinement erklärt. Durch den niedrigeren Synthesedruck und den veränderten MAA-Gehalt wurde aus den statistisch eingebauten Monomereinheiten ein geordnetes Blocksystem (ABABAB...). Da die einzelnen Monomere verschiedene chemische Eigenschaften zeigen 


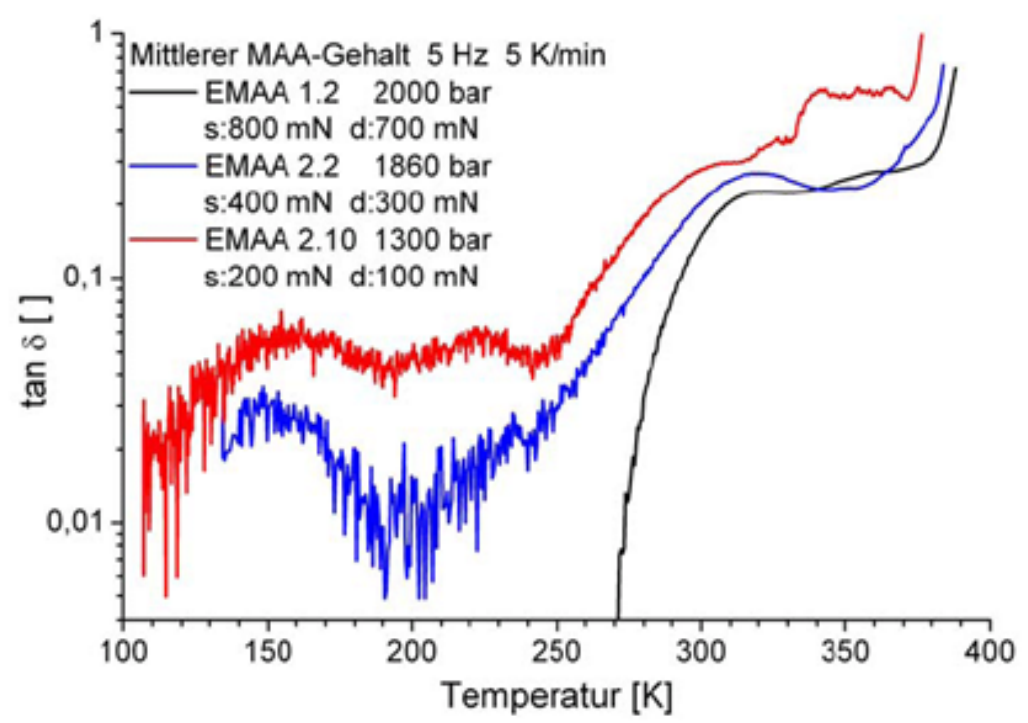

Abbildung 1.25: Zusätzlicher Chemical Confinement Peak unterhalb von $\mathrm{T}_{G}{ }^{[14]}$

(Acrylsäure besitzt die Möglichkeit, Wasserstoffbrückenbindungen auszubilden, Ethylen nicht), können sich durch die blockweise auftretenden Monomere neue Eigenschaften im Polymer bilden. Über inter- und intramolekulare Wasserstoffbrücken werden Polymereinheiten festgehalten, so dass zwischen diesen Einheiten Teilstücke entstehen, die wie im Kapitel 1.5.1 Kurbelwellenbewegungen hervorrufen können (siehe Abb. 1.26).

Es ist jedoch nicht abschließend geklärt, ob die Idee des Chemical Confinements ein allgemein gültiges Charakteristikum von Blockcopolymeren darstellt oder ein Sonderfall für diese Versuche war.

a)

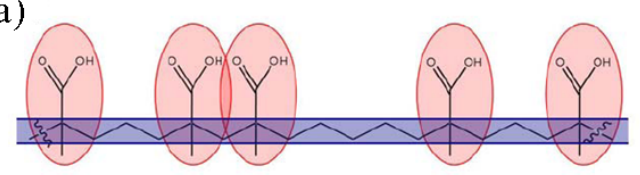

b)

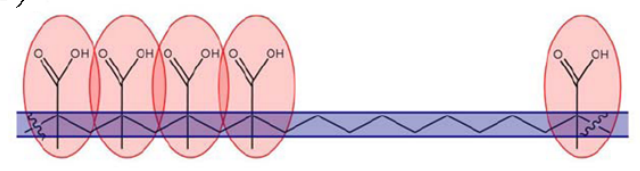

a')

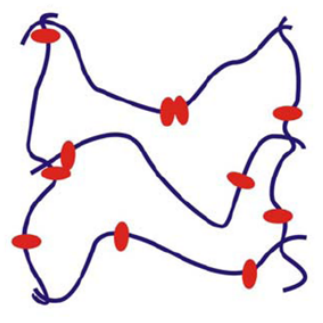

b')

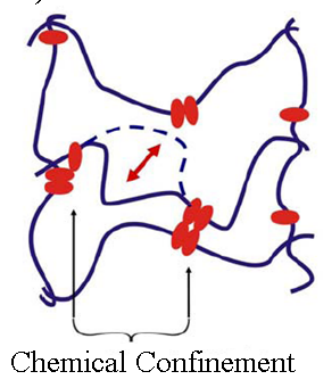

Abbildung 1.26: Schematische Darstellung der Idee des Chemical Confinements: a) statistisches Polymer mit a') Verteilung der möglichen Wasserstoffbrücken, b) blockweise aufgebautes Polymer mit b') Wasserstoffbrücken, die ein Chemical Confinement bilden. ${ }^{[14]}$ 



\section{Material und Methoden}

In diesem Kapitel werden sowohl die verwendeten Materialien und Geräte als auch die Herstellung der Proben beschrieben. Außerdem werden Spezifikationen der Messungen und Auswertungen erklärt. Dabei wird zwischen den metallischen Proben (Kapitel 2.1) und den Polymerproben (Kapitel 2.2) unterschieden.

\subsection{Metallische Proben}

Um metallische Gläser anzufertigen, werden zunächst homogene Vorlegierungen aus den entsprechenden elementaren Reinsnmaterialien in einem Lichtbogenofen (Typ MAM-1 der Firma Bühler) hergestellt. Die verwendeten Metalle sind in Tabelle 2.1 aufgelistet.

\begin{tabular}{|c|c|c|c|}
\hline Element & Hersteller & Form & Reinheit \\
\hline Aluminium & Alfa Aesar & Stange & $4 \mathrm{~N} 8$ \\
\hline Kupfer & Strem Chemicals & Granulat & $5 \mathrm{~N}$ \\
\hline Palladium & Goodfellow & Granulat & $3 \mathrm{~N} 5$ \\
\hline Silizium & Alfa Aesar & Block & $6 \mathrm{~N}$ \\
\hline Zirkonium & Wah Chang & Block & $5 \mathrm{~N}$ \\
\hline
\end{tabular}

Tabelle 2.1: Verwendete Metalle zur Herstellung der metallischen Gläser

Die noch kristallinen Vorlegierungen werden dann über Melt Spinning zu amorphen Bändern weiterverarbeitet, welche typischerweise eine Breite von 1,1 mm - 2,5 mm und eine Dicke von 0,02 mm - 0,05 mm besitzen. Die genaue Herstellungsweise ist in ${ }^{[14]}$ beschrieben und eine gute theoretische Einführung geben Jones et al. ${ }^{[76]}$ und Greer et al. ${ }^{[7]}$ Die Zusammensetzungen sind in Tabelle 2.2 aufgeführt. 


\begin{tabular}{|c|c|c|c|}
\hline Probe & Palladium & Kupfer & Silizium \\
\hline Soll-Zusammensetzung [at\%] & 77,5 & 6,0 & 16,5 \\
\hline Ist-Zusammensetzung [at\%] & 77,8 & 6,2 & 16,0 \\
\hline Probe & Zirkonium & Aluminium & Kupfer \\
\hline Soll-Zusammensetzung [at\%] & 65 & 7,5 & 27,5 \\
\hline Ist-Zusammensetzung [at\%] & 65,8 & 7,1 & 27,1 \\
\hline
\end{tabular}

Tabelle 2.2: Zusammensetzungen der metallischen Proben - die Palladium-KupferSiliziumproben wurden von Jörg Hachenberg übernommen ${ }^{[14]}$

Um auszuschließen, dass die Proben noch kristalline Bereiche enthalten, wird die Atomstruktur über Weitwinkelröntgenanalysen untersucht. Hierfür wird ein Diffraktometer des Typs D5000 von Siemens verwendet. Eine theoretische Beschreibung findet man hierzu in Cullity et al. ${ }^{[78]}$ und Hachenberg et al. ${ }^{[14]}$

\subsubsection{Mechanische Messungen von metallischen Proben}

Zur Analyse von viskoelastischen Eigenschaften der Proben wurde ein dynamischmechanischer Analysator (DMA 7 von PerkinElmer) verwendet. Dieser bietet eine Vielzahl von Messmöglichkeiten, die von einfachen Zugversuchen über Creep/Recoverymessungen bis zu Messungen mit einer sich ändernden oszillierenden Spannung reicht (siehe Kapitel 1.3.1). In diesem Kapitel wird kurz der Aufbau und die Handhabung der DMA beschrieben, mit Fokus auf die entsprechenden Einstellungen bei den Messungen. Die Steuerung der DMA erfolgt mit Hilfe eines integrierten Messprogrammes und Windows-basierter Software. Die Ansteuerung der DMA und das Auslesen der Messgrößen erfolgt durch ein Steuergerät vom Typ TAC 7/DX, das seinerseits mit einem Computer verbunden ist. Auf diese Weise werden alle programmierbaren Parameter und die detektierten Größen aufgezeichnet. Zur Auswertung der Messgrößen wurde Origin 8.0 verwendet.

In Abbildung 2.1 ist der Aufbau der DMA schematisch dargestellt. In der Vergrößerung kann man die verwendete Probenaufhängung für metallische Bänder erkennen.

Die benötigte Kraft (maximal 1 N) wird über eine Spule, welche mit einem Magneten gekoppelt ist, auf den Sondenstab übertragen und direkt auf die Probe umgelegt, welche 

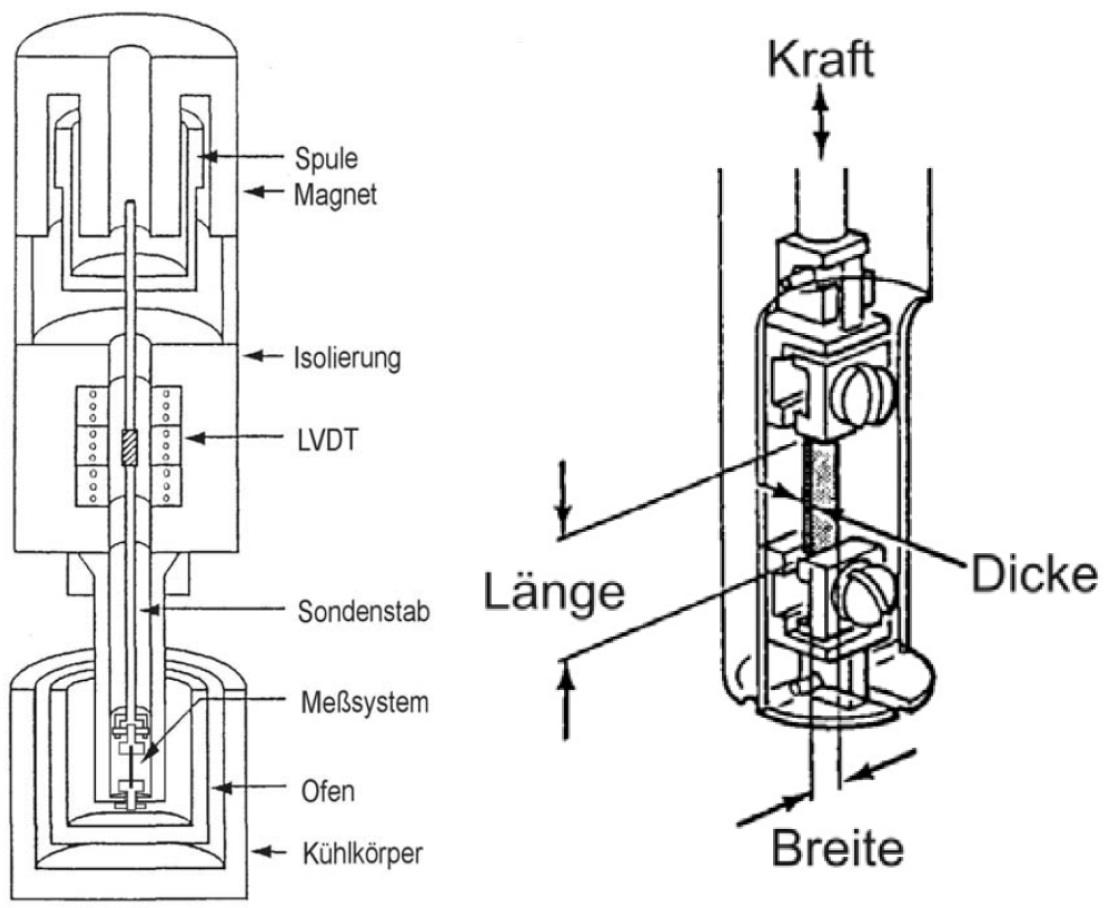

Abbildung 2.1: Schematische Abbildung der DMA mit Vergrößerung der Probenaufhängung zur Vermessung der metallischen Bänder ${ }^{[79]}$

über zwei Klemmen in der DMA-Halterung eingespannt ist. Die Probenlänge wird über einen Linear Variablen Differential-Transformator (LVDT) detektiert. Während des Versuches kann die Probe innerhalb eines Ofens sowohl gekühlt als auch geheizt werden, dabei wird über einen Stickstoffstrom (Reinheit 4.0) im Sondenrohr der LVDT vom Ofen entkoppelt. Maximal können Temperaturen von ca. 770 K erreicht werden. Gekühlt wird entweder mit einem Glykol-Durchflusskühler (bis ca. $270 \mathrm{~K}$ ) oder, wenn tiefere Temperaturen zu erreichen sind, durch flüssigen Stickstoff (bis ca. 150 K, Steuerung der Durchflussreglung durch ein Steuergerät mit Temperaturfühler vom Typ CCA 7). Bei Temperaturen unter $250 \mathrm{~K}$ ist zur Vermeidung von Kondenswasser die Verwendung einer Handschuhbox mit Stickstoffatmosphäre notwendig, wodurch auch Oxidationseffekte vermieden werden können. Die Kontrolle von Wasser- und Sauerstoffgehalt in der Handschuhbox erfolgt durch eine Anlage vom Typ MB 20G von MBraun. Es wurde in der Regel zu Beginn der Messung eine Wasserkonzentration von unter 20 ppm und eine Sauerstoffkonzentration von unter 50 ppm erreicht (minimal ist eine Konzentration von unter 5 ppm möglich). In den folgenden drei Unterkapiteln werden die verwendeten Messspezifikationen der DMA beschrieben. 


\subsubsection{Dynamisch-mechanische Analysen}

Wie im Kapitel 1.3.1 beschrieben, wird bei dynamischen Messungen eine größere statische Kraft von einer kleineren dynamischen überlagert. Bei den in Kapitel 3 vorgestellten Messungen wurde die statische Kraft zwischen $250 \mathrm{mN}$ und $500 \mathrm{mN}$ und die dynamische zwischen $200 \mathrm{mN}$ und $450 \mathrm{mN}$ mit einer Frequenz zwischen $1 \mathrm{~Hz}$ und $5 \mathrm{~Hz}$ gewählt. Die Heizrate während der Versuche betrug zwischen $0,1 \mathrm{~K} / \mathrm{min}$ und $20 \mathrm{~K} / \mathrm{min}$. Die genauen Messparameter sind jeweils bei den Einzelmessungen explizit angegeben.

\subsubsection{Aufnahme von Spannungs-Dehnungskurven}

Spannungs-Dehnungskurven, durchgeführt in der DMA 7, wurden in dieser Arbeit spannungskontrolliert über eine konstante Kraftänderung aufgenommen (siehe Kapitel 1.3.1.2). Es wurden verschiedene Kraftänderungen im Bereich von $1 \mathrm{mN} / \mathrm{min}$ bis $50 \mathrm{mN} / \mathrm{min}$ bei konstanter Temperatur aufgenommen. Mit Hilfe einer Zwick Materialprüfmaschine ${ }^{1}$ konnten ebenfalls dehnungskontrollierte Analysen bei Raumtemperatur vorgenommen werden, wobei die Dehnungsrate bei $0,03 \mathrm{~mm} / \mathrm{h}\left(8,3 \cdot 10^{-6} \mathrm{~mm} / \mathrm{s}\right) \mathrm{lag}$. Diese Messungen wurden in Kooperation mit der Arbeitsgruppe von Professor Vana ${ }^{2}$ durchgeführt. Die genauen Vorgaben zu Kraft, Dehnung und Temperatur sind bei den Einzelmessungen aufgeführt.

\subsubsection{Analysen von Creep/Recoverykurven}

Die Messungen von Creep/Recoverykurven wurden bei zwei unterschiedlichen Temperatureinstellungen vorgenommen. Zum einen wurde bei konstanter Temperatur gemessen und zum anderen mit einer konstanten Heizrate. Bei den Messungen mit konstanter Temperatur wurde die Delay-time von PdCuSi in $25 \mathrm{~K}$ Schritten von Raumtemperatur bis zu $550 \mathrm{~K}$ und mit einer Kraft von $50 \mathrm{mN}$ bis maximal $950 \mathrm{mN}$ aufgenommen. Dazu wurden die Kraftsprünge bei jeder Temperatur sechsmal wiederholt, wobei die Kraft zwischen den Sprüngen für 5 min. gehalten wurde. Aus den Wiederholungen wurde der Mittelwert gebildet. Die Kraftunterschiede lagen immer bei +/-200 mN zum Mittelwert,

${ }^{1}$ zwicki-Line der Firma Zwick GmbH \& Co. KG

${ }^{2}$ Institut für Funktionelle Polymermaterialien der Fakultät für Chemie der Georg-August-Universität Göttingen, Tammannstr. 4, 37077 Göttingen 
so dass bei einer Messung in der Regel immer ein Hub von 400 mN vorlag (Ausnahme bei der Messung siehe Abb. 3.20, es wurde als untere Kraft immer $50 \mathrm{mN}$ eingestellt und nur die maximale Kraft verändert, so dass bei jeder Messung ein unterschiedlicher Hub vorlag).

Bei den Messungen mit einer konstanten Heizrate wurde von Raumtemperatur bis $670 \mathrm{~K}$ (PdCuSi) und von $340 \mathrm{~K}$ bis $520 \mathrm{~K}(\mathrm{ZrAlCu})$ mit $0,78 \mathrm{~K} /$ min geheizt. Die Kräfte wurden wie oben beschrieben mit einem Hub von 400 mN zwischen Creep- und Recoveryteil eingestellt, wobei ebenfalls in $50 \mathrm{mN}$ Abständen (von $50 \mathrm{mN}$ bis maximal $950 \mathrm{mN}$ ) zwischen den einzelnen Messungen gearbeitet wurde. Bei jeder Messung wurden 300 Wiederholungen aufgenommen, mit einer Haltezeit von 1,1 min im Creepteil und 0,5 min im Recoveryteil. Aus dem linearen Anteil nach dem Kraftsprung (15 Datenpunkte bei einer Aufnahmegeschwindigkeit von 0,2 s zwischen den Datenpunkten entsprechen $3 \mathrm{~s}$ ) wurde sowohl der Elastizitätsmodul E als auch das Aktivierungsvolumen $\Omega^{*}$ bestimmt. Die Auswertung der Creep/Recovery-Messungen wurde mit Hilfe eines von Friedrich Romstedt $^{3}$ geschriebenen Programms und Origin 8.0 vorgenommen. Der Quelltext des Programms ist im Anhang aufgeführt (siehe Kapitel 6).

Zur Berechnung des Aktivierungsvolumens mit Hilfe der Gleichung 1.14 müssen vier Näherungen angenommen werden.

1. Die Gleichung 1.14 gilt für eine konstante Dehnungsrate. Dies ist in diesen Versuchen nicht gegeben, da die Kraft in einem Sprung verändert wird. Um aber trotzdem die Gleichung 1.14 verwenden zu können, wird das Aktivierungsvolumen nur im linearen Bereich (3 s direkt nach dem Kraftsprung) der Creep/Recovery-Kurven bestimmt. Hier gilt ein linearer Zusammenhang zwischen der Spannungsänderung und der fiktiven Dehnungsrate $\dot{\epsilon}$, welche über

$$
\dot{\epsilon}=\frac{\epsilon}{t}
$$

berechnet wird.

\footnotetext{
${ }^{3}$ Friedrich Romstedt, Institut für Astrophysik der Fakultät für Physik der Georg-August-Universität, Friedrich-Hund-Platz 1, 37077 Göttingen
} 
Dabei ist $\epsilon$ die relative Dehnungsänderung, bestimmt über

$$
\epsilon=\frac{\Delta l}{l}
$$

mit 1 der Probenlänge und t dem verwendeten Zeitintervall von $3 \mathrm{~s}$.

2. Die Gleichung 1.14 gilt nur bei einer festen Temperatur. Da die Heizrate nur $0,78 \mathrm{~K} / \mathrm{min}$ betrug, ist es zulässig, in dem verwendeten Zeitintervall die Temperatur als konstant anzunehmen.

3. Es wurde der Betrag des Aktivierungsvolumens verwendet.

4. Es wird angenommen, dass das aktivierte Volumen sich sphärisch verhältt ${ }^{[45,69]}$, so dass über das Kugelvolumen sowohl der Durchmesser als auch die Atommenge bestimmt werden kann.

\subsection{Polymerproben}

Die ABA-Block-Copolymere wurden in Zusammenarbeit mit der Arbeitsgruppe von Professor Vana ${ }^{4}$ innerhalb des Graduiertenkollegs 782 hergestellt. Es wurde zur Polymersynthese eine „Reversible addition-fragmentation chain transfer (RAFT) - Polymerisation“ durchgeführt, wozu das RAFT-Agenz Dimethyl-2,6-di(propyltrithiocarbonyl)heptandioat (DPTH) (siehe Abb. 2.2) synthetisiert wurde.<smiles>CCCSC(=S)SC(CCCC(SC(=S)SCCC)C(=O)OC)C(=O)OC</smiles>

Abbildung 2.2: Verwendetes RAFT-Agenz zur Herstellung der Block-Copolymere

${ }^{4}$ Institut für Funktionelle Polymermaterialien der Fakultät für Chemie der Georg-August-Universität Göttingen, Tammannstr. 4, 37077 Göttingen 
Folgend wurden über eine RAFT-Polymerisation ${ }^{[80-83]}$ der innere Block (Poly(tert.butylacrylat) siehe Abb. 2.3) und anschließend die äußeren Blöcke (Poly(tert.-butylacrylat) mit 5 Gew.\% Acrylsäure) hergestellt.

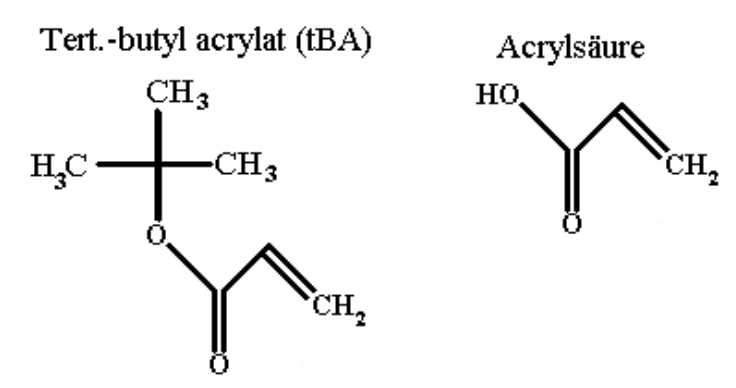

Abbildung 2.3: Verwendete Monomereinheiten

Die zur Polymerisation verwendeten Chemikalien sind in der folgenden Tabelle 2.3 aufgeführt:

\begin{tabular}{|c|c|}
\hline Chemikalie & Hersteller \\
\hline Natriummethoxid (30 gew.\% in Methanol) & Fluka \\
\hline 1-propanthiol & Aldrich \\
\hline Kohlenstoffdisulfid & Fluka \\
\hline Dimethyl-2,6-dibromoheptandioat & Aldrich \\
\hline Acrylsäure & Fluka \\
\hline 2,2'-Azo-bis-isobutyronitril $($ AIBN $)$ & AKZO \\
\hline Tert.-butyl acrylat $(t \mathrm{BA})$ & Fluka \\
\hline
\end{tabular}

Tabelle 2.3: Verwendete Chemikalien und Hersteller

Für die DPTH Synthese wurde eine Lösung aus Natriummethoxid in Methanol tropfenweise bei Raumtemperatur zu 1-Propanthiol im Molverhältnis von $1: 1$ gegeben, für eine Stunde gerührt und anschließend Kohlenstoffdisulfid (Molverhältnis zu DPTH 1 : 1,3) langsam zugegeben. Nach weiteren 5 Stunden wurde Dimethyl-2,6-dibromoheptanedioat (Molverhältnis zu DPTH 1 : 0,5) hinzugefügt und weitere 16 Stunden gerührt, wonach das Lösungsmittel unter Vakuum entfernt wurde. Der Rückstand wurde anschließend mit Dichloromethan gewaschen, filtriert und erneut unter Vakuum getrocknet. Hiernach wurde zur Herstellung des inneren Blockes eine Lösung aus DPTH, AIBN und $t$ BA (mit 
einem Molverhältnis zueinander von ca. $10: 1: 1$ ) in Toluol bei $60{ }^{\circ} \mathrm{C}$ Reaktionstemperatur verwendet. Durch Abschrecken in Eiswasser wurde die Polymerisation gestoppt, so dass über die Reaktionszeit der Umsatz gesteuert werden konnte.

Die Synthese der ABA-Block-Copolymere wurde identisch wie die Herstellung des inneren Blockes durchgeführt, wobei die Lösung aus AIBN, DPTH, tBA und Acrylsäure im Molverhältnis von ca. $1: 1: 3120: 28$ eingestellt wurde. Die genauen Mengenangaben und Reaktionsbedingungen können bei Schwabe et al. nachgelesen werden. ${ }^{[84]}$

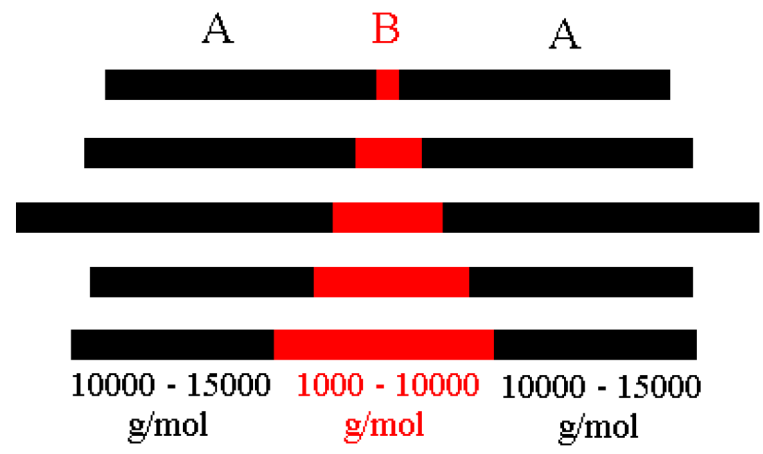

Abbildung 2.4: Schematische Abbildung der ABA-Block-Copolymere

Es wurden ABA-Block-Copolymere in der Größenordnung von ca. 30000 g/mol hergestellt. Der innere Block B wurde dabei von $1000 \mathrm{~g} / \mathrm{mol}$ - $10000 \mathrm{~g} / \mathrm{mol}$ und die äußeren Blöcke A von $10000 \mathrm{~g} / \mathrm{mol}$ - $15000 \mathrm{~g} / \mathrm{mol}$ variiert (siehe Abb. 2.4), so dass die Abhängigkeit der Relaxationen vom inneren als auch von den äußeren Blöcken systematisch untersucht werden konnte.

\subsection{Charakterisierung der Polymerproben}

Zur Charakterisierung der Polymerproben wurden die Molekulargewichtsverteilung bestimmt, Röntgenspektren aufgenommen und DSC-Messungen durchgeführt.

Die Molekulargewichtsverteilung der Polymere wurde über Size Exclusion Chromatography (SEC) unter Verwendung von einem JASCO AS-2055-plus Autosampler, einer Waters 515 HPLC Pumpe, drei PSS-SDV Kolonnen mit einer Partikelgröße von ca. $5 \mathrm{~nm}$ und Porengrößen von $10^{5} \AA, 10^{3} \AA$ and $10^{2} \AA$ und einem Waters 2410 BrechungsindexDetektor bestimmt. Als Lösungsmittel wurde THF bei $35^{\circ} \mathrm{C}$ mit einer Durchflussrate von $1 \mathrm{~mL} / \mathrm{min}$. verwendet. 
Zur Bestimmung der Amorphizität wurden zum einen Weitwinkelröntgenanalysen durchgeführt, wobei ein Diffraktometer des Typs D5000 von Siemens verwendet wurde. Zum anderen wurde die Differential Scanning Calorimetry (DSC, DSC 7 von PerkinElmer) verwendet, um die Glasübergangstemperatur zu bestimmen und diese mit mechanisch-dynamischen Anlaysen vergleichen zu können. Für die DMA-Messungen wurde eine DMA 7 von PerkinElmer verwendet (siehe Kapitel 2.4).

\subsection{Dynamisch-mechanische Analysen der Polymerproben}

Nach der Polymerherstellung wurden die Proben in einer hydraulischen Presse zu Zylindern mit einem Durchmesser von 6 mm und einer Höhe von 2 mm -6 mm verarbeitet. Gepresst wurde mit einer selbst hergestellten Halterung aus gehärtetem Stahl und einem Druck von 1,25 kPa. Die Polymerzylinder wurden in einem Aluminiumbehälter innerhalb der DMA vermessen, wobei sie in eine zylindrische Vertiefung hineingelegt und mit einem Deckel bedeckt wurden, welcher die Kraft auf die Probe überträgt.

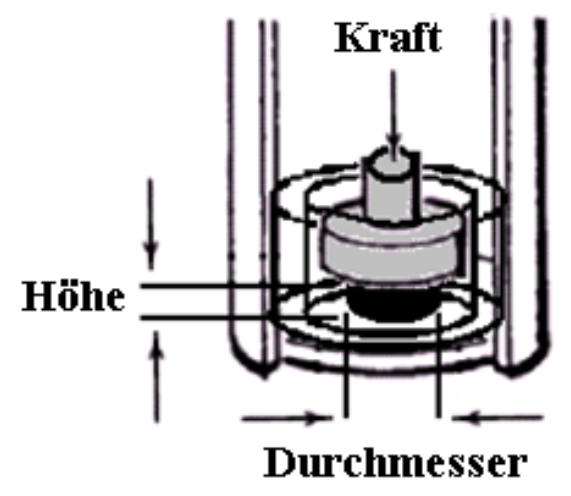

Abbildung 2.5: Probenauflage zur Vermessung der Polymerproben

Die verwendeten Deckel passten ohne Reibung in das Probenloch, da ein Abstand zur Wand von ca. 0,2 mm gewählt wurde. Die Probe wurde ebenfalls mittig in das Loch plaziert, so dass kein Wandkontakt bestand und der Deckel die gesamte Probenoberfläche bedeckte. Die Probenaufnahme im Hohlzylinder hat einen runden, ebenen Boden mit einem Durchmesser und einer Höhe von je 8 mm (siehe Abb. 2.5). Die Analysen wurden alle unter denselben Messbedingungen durchgeführt. Es wurde eine statische Kraft von 
$500 \mathrm{mN}$, eine dynamische von $400 \mathrm{mN}$ und eine Frequenz von $5 \mathrm{~Hz}$ gewählt. Gemessen wurde von $150 \mathrm{~K}$ bis maximal $420 \mathrm{~K}$ mit einer Heizrate von $1 \mathrm{~K} / \mathrm{min}$. Zum Vergleich wurden DSC-Spektren mit einer Heizrate von $5 \mathrm{~K} / \mathrm{min}$ aufgenommen. 


\section{Ergebnisse}

In diesem Teil der Arbeit werden die Ergebnisse vorgestellt und beschrieben. Dabei ist dieses Kapitel in die Unterkapitel Relaxationen in amorphen Metallen (3.1) und Relaxationsprozesse von ABA-Block-Copolymeren (3.2) eingeteilt. Es werden im ersten Teil sowohl Untersuchungen an einem fragilen ( $\mathrm{PdCuSi}$ ) als auch an einem starken (ZrAlCu) Glasbildner dargestellt, wobei der Fokus auf PdCuSi liegt. Im zweiten Teil werden dann die Ergebnisse von den Block-Copolymeren (siehe auch Kapitel 2.2) gezeigt.

\subsection{Relaxationen in amorphen Metallen}

In der Literatur werden verschiedene Relaxationsprozesse für metallische Gläser diskutiert, die den Übergang vom amorphen, festen Zustand in die unterkühlte Schmelze beschreiben (siehe Kapitel 1.4). Rösner et al. und Hachenberg et al. konnten das Auftreten der makroskopischen Relaxationsprozesse sowohl für starke als auch für fragile

Glasbildner zeigen. ${ }^{[13,14]}$ In dieser Arbeit liegt der Schwerpunkt auf der Untersuchung des Überganges vom Glaszustand in die unterkühlte Schmelze, welcher in SpannungsDehnungskurven als der Übergang vom elastischen zum plastischen Bereich beschrieben wird (siehe Kapitel 1.3.1.2). Dafür werden zuerst allgemein die Relaxationsprozesse im Glaszustand (siehe Kapitel 3.1.1) und im Speziellen der Übergang in die unterkühlte Schmelze untersucht.

\subsubsection{Heizratenabhängige Relaxationen}

Die heizratenabhängige Verschiebung der Relaxationsprozesse bis hin zum Merging war in den vergangenen Jahren ein wichtiges Thema in der Literatur ${ }^{[8,14,39,85-89]}$. Dabei fanden jedoch sehr langsame Heizraten von unter $1 \mathrm{~K} / \mathrm{min}$ kaum Beachtung. In diesem Unterkapitel soll diese Lücke nun geschlossen werden. 
Am fragilen Glasbildner PdCuSi wurde der Verlauf des Speicher- und Verlustmoduls bei verschiedenen Heizraten verglichen. Hierfür wurden dynamische Messungen mithilfe der DMA 7 durchgeführt, wobei eine statische Kraft von $250 \mathrm{mN}$ und eine dynamische von $200 \mathrm{mN}$ verwendet wurde. Gearbeitet wurde bei einer Frequenz von $1 \mathrm{~Hz}$ und einer Aufnahmegeschwindigkeit von einem Datenpunkt pro Sekunde, wobei von Raumtemperatur bis über die Kristallisation hinaus gemessen wurde. In Abbildung 3.1 sind die Speicher- (a) und die Verlustmodule (b) dargestellt.

a)

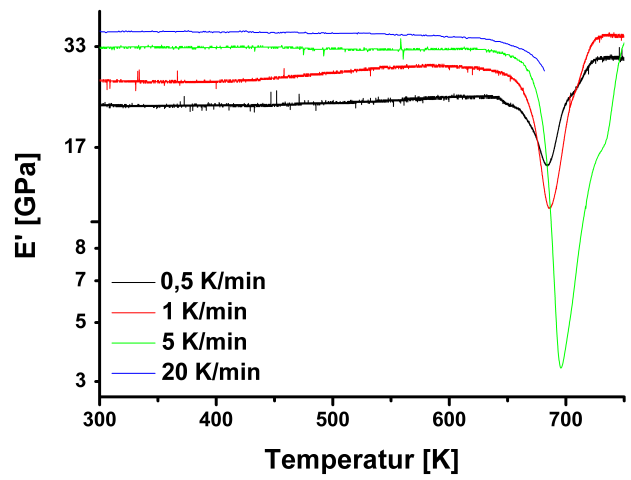

b)

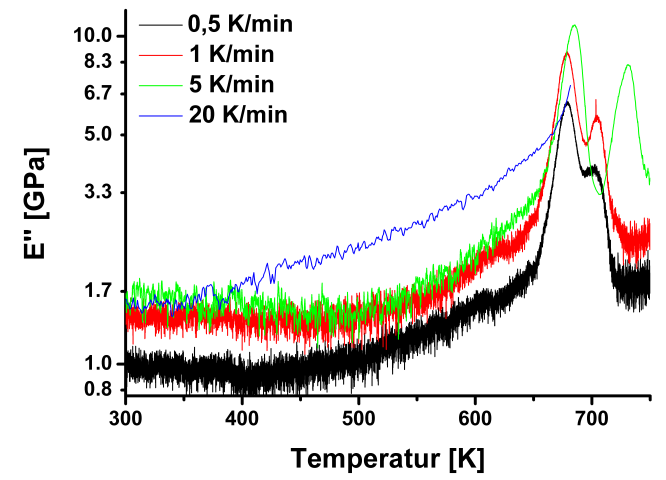

Abbildung 3.1: Vergleich von a) Speicher- und b) Verlustmodul bei verschiedenen Heizraten

Zum Vergleich wurden die Heizraten $20 \mathrm{~K} / \mathrm{min}$ (blau - Probe gerissen bei $680 \mathrm{~K}$ ), $5 \mathrm{~K} / \mathrm{min}$ (grün), $1 \mathrm{~K} / \mathrm{min}$ (rot) und $0,5 \mathrm{~K} / \mathrm{min}$ (schwarz) untersucht, wobei für jede Messung eine neue Probe verwendet wurde. Im Bildteil a) ist zu erkennen, dass der niedrigste Anfangsmodul von 22 GPa bei der kleinsten Heizrate bestimmt wurde. Der höchste Anfangsmodul (37 GPa) konnte bei der größten Heizrate gefunden werden. Der beobachtete Verlauf der Module ist bei allen Messungen sehr ähnlich. Nach einem annähernd konstanten Verhalten ist bei ca. $645 \mathrm{~K}$ ein starkes Abknicken zu beobachten, wonach ein Minimum durchlaufen wird und der Modul wieder ansteigt. Hierbei ist jedoch zu bemerken, dass sich sowohl der Abknickpunkt $\left(\mathrm{T}_{G}\right)$ als auch das Minimum (Kristallisation) mit größerer Heizrate zu höheren Temperaturen und das Minimum zu niedrigeren Werten im Modul verschiebt. Bei den Kurven der Heizraten 0,5 K/min, $1 \mathrm{~K} / \mathrm{min}$ und $5 \mathrm{~K} / \mathrm{min}$ ist im ansteigenden Teil der Kurve eine „Schulter“ zu erkennen, welche durch weitere Kristallisationsstufen hervorgerufen wird. 
Im zweiten Bildteil b) sind die bestimmten Verlustmodule zu sehen. Mit zunehmender Heizrate nimmt ebenfalls der Anfangswert zu (1 GPa - 1,6 GPa). Nach einem kurzen konstanten Verlauf steigen alle Module zum Wing an, wobei der Abknickpunkt nicht offensichtlich mit Erhöhung der Heizrate korreliert. Jedoch ist die Höhe des Moduls im Wing abhängig von der Heizrate, wobei der kleinste Modul bei der niedrigsten Heizrate bestimmt wurde. Bei ca. $650 \mathrm{~K}$ kann ein erneutes Ansteigen $\left(\mathrm{T}_{G}\right)$ im Imaginärteil beobachtet werden, woraufhin der $\alpha$-Peak und die überlagerte Kristallisation zu sehen sind (außer bei $20 \mathrm{~K} / \mathrm{min}$, da die Probe zuvor reißt). Die Glasübergangstemperatur verschiebt sich hierbei wie auch das beobachtete Maximum mit höherer Heizrate zu höheren Temperaturen. Nach Durchlaufen des Maximums fällt der Modul wieder ab, um daraufhin ein zweites Maximum zu durchlaufen. Dieses (entsprechend der Schulter im Realteil) ist mit zunehmender Heizrate ausgeprägter und ebenfalls zu höheren Temperaturen verschoben (Kristallisation).

Zur weiteren Untersuchung des heizratenabhängigen Verhaltens wurde die Heizrate um den Faktor 5 auf $0,1 \mathrm{~K} / \mathrm{min}$ verringert. Hierbei wurde ebenfalls eine statische Kraft von $250 \mathrm{mN}$ und eine dynamische von $200 \mathrm{mN}$ verwendet, jedoch bei einer Frequenz von $2 \mathrm{~Hz}$ und einer Aufnahmegeschwindigkeit von einem Messpunkt pro 5 Sekunden gemessen. Es wurden vier Durchläufe untersucht, wobei jeweils von Raumtemperatur auf $600 \mathrm{~K}$ erwärmt und dann mit $100 \mathrm{~K} /$ min abgekühlt wurde. Beim vierten Durchlauf wurde jedoch über die Kristallisation hinaus bis auf $720 \mathrm{~K}$ erwärmt.

In Abbildung 3.2 sind die vier Durchläufe zu erkennen. In blau ist jeweils der Speicherund in rot der Verlustmodul abgebildet. Im ersten Durchlauf (Bildteil a) wurde ein Anfangswert für den Realteil von 20,5 GPa gemessen, welcher bis $450 \mathrm{~K}$ konstant bleibt und dann auf $48 \mathrm{GPa}$ ansteigt. Der Imaginärteil beginnt bei 1,1 GPa, fällt dann zunächst ab, um ab $400 \mathrm{~K}$ ebenfalls anzusteigen. Bemerkenswert ist, dass hierbei ein Maximum bei $525 \mathrm{~K}$ durchlaufen wird, um folgend weiter anzusteigen. Im zweiten Durchlauf (Bildteil b) ist zu erkennen, dass der Speichermodul beim selben Wert beginnt, wie er beim ersten Durchlauf endete. Es ist ein leicht fallender Verlauf bis $550 \mathrm{~K}$ zu erkennen, woraufhin der Modul auf 35,7 GPa ansteigt. Der Verlustmodul hat einen Anfangswert wie im ersten Durchlauf, es ist jedoch kein Abfallen zu erkennen, sondern ein sofortiger Anstieg bis $600 \mathrm{~K}$, worauf ebenfalls ein Maximum durchlaufen wird (Maximum bei $540 \mathrm{~K}$ ). 


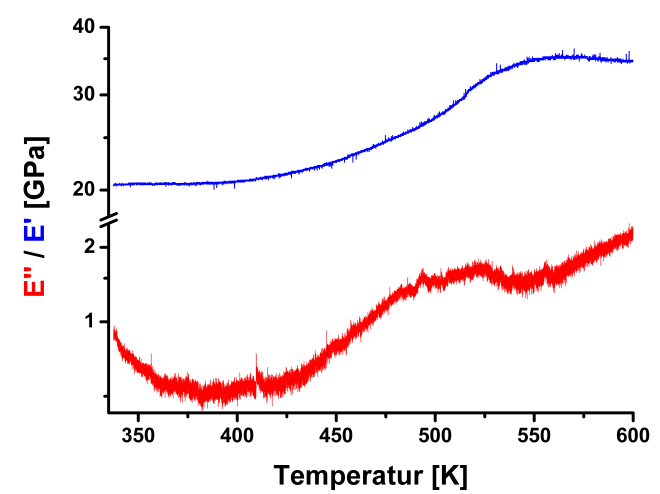

c)

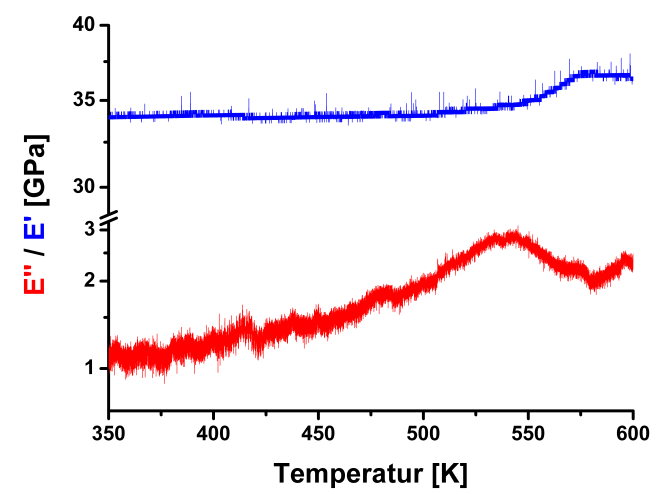

b)

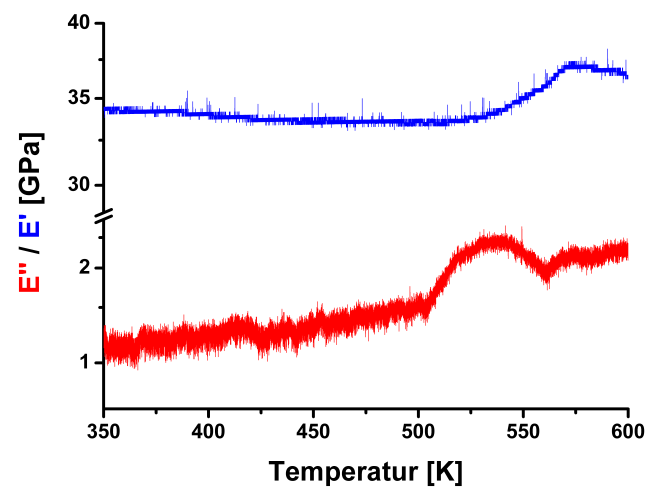

d)

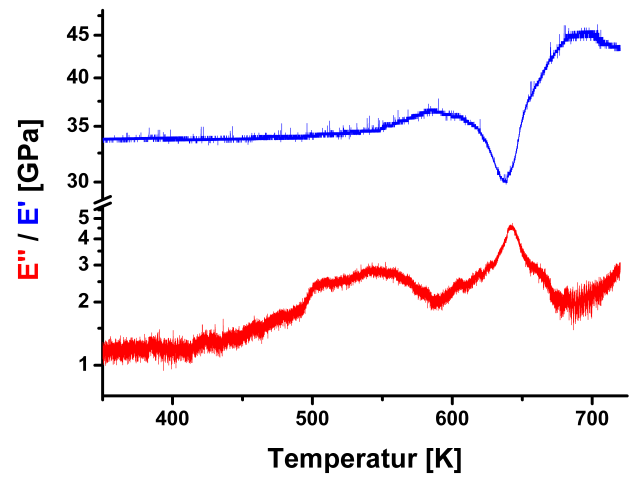

Abbildung 3.2: Vergleich der Moduli vom a) ersten, b) zweiten, c) dritten und d) vierten Durchlauf der Untersuchung des Relaxationsverhaltens bei einer Heizrate von $0,1 \mathrm{~K} / \mathrm{min}$

Der dritte und vierte Durchlauf ist in den Bildteilen c) und d) zu sehen. Die vorletzte Messung (Bildteil c) ähnelt der zweiten sehr stark. Der Speichermodul beginnt bei 34,3 GPa und steigt ab $550 \mathrm{~K}$ an. Im Verlustmodul ist ebenfalls ab Beginn der Messung ein Ansteigen und auch weiterhin ein verschmierter Peak (Maximum bei $540 \mathrm{~K}$ ) zu beobachten. Der letzte Durchlauf (Bildteil d) ist identisch zum dritten bis hin zu 600 K. Danach wurde nicht wieder abgekühlt, sondern weiter erwärmt. So ist bei höheren Temperaturen im Realteil ein Abfallen des Moduls zu erkennen, worauf der Modul ab $640 \mathrm{~K}$ wieder ansteigt und scheinbar einen stabilen Wert von ca. 43 GPa $\left(\Delta \mathrm{E}^{\prime}=12 \mathrm{GPa} \hat{=} 28 \%\right)$ erreicht. Im Verlustmodul ist nach $600 \mathrm{~K}$ ein starker Anstieg mit einem Maximum bei ebenfalls $640 \mathrm{~K}$ zu beobachten, worauf der Modul bis $680 \mathrm{~K}$ abfällt und daraufhin langsam wieder ansteigt.

Die aufgenommene Probenlänge aller vier Durchgänge ist in Abbildung 3.3 dargestellt. Bei deren Vergleich ist zu erkennen, dass sich die Probe linear mit der Temperatur bis ca. $565 \mathrm{~K}$ (erster Durchlauf) und $600 \mathrm{~K}$ (bei den weiteren Durchläufen) ausdehnt, wobei 


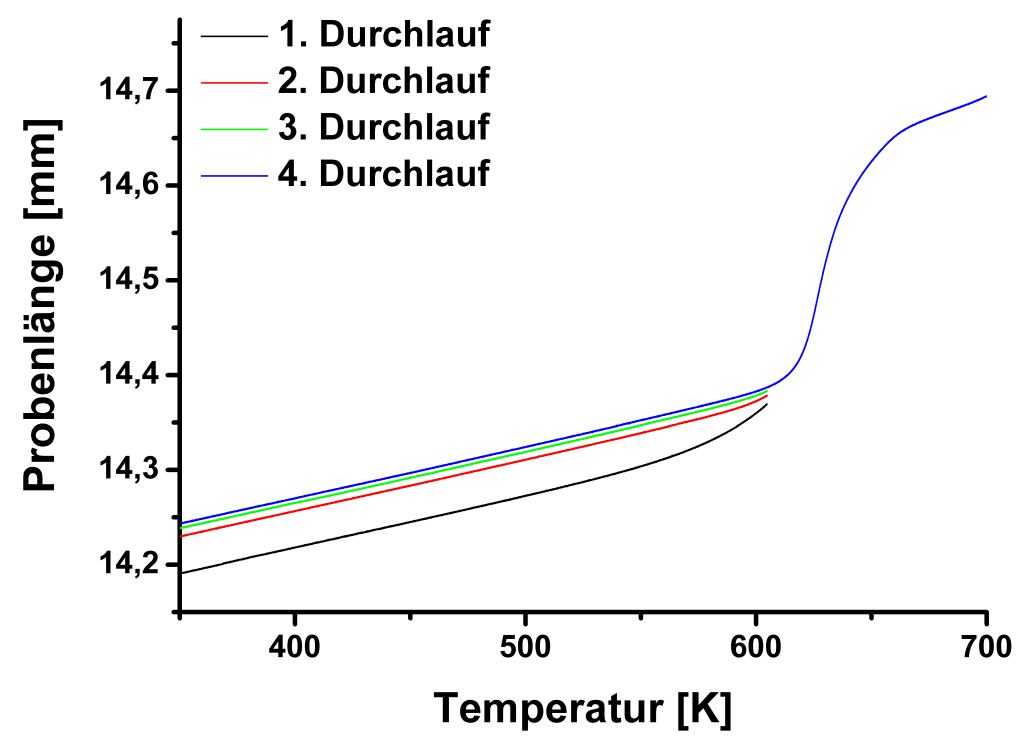

Abbildung 3.3: Vergleich der Probenlänge der vier Durchläufe

im ersten Durchlauf die Probe deutlich kürzer ist. Im Temperaturbereich über $600 \mathrm{~K}$ ist im vierten Durchlauf eine starke Längenänderung zu erkennen (viskoses Fließen), wobei bei $630 \mathrm{~K}$ der stärkste Anstieg erreicht ist. Ab $670 \mathrm{~K}$ ist wieder eine geringere Steigung wie vor dem Anstieg zu beobachten.

In Abbildung 3.4 ist eine Gegenüberstellung der Module des vierten Durchgangs (rot und blau) mit der Messung der kristallisierten Probe (grün und grau) zu sehen, wobei deutliche Unterschiede zu erkennen sind. Der Speichermodul der kristallinen Probe beginnt bei einem Wert von 39 GPa, läuft konstant bis zu $660 \mathrm{~K}$, worauf dieser ansteigt. Ein Maximum wird bei $680 \mathrm{~K}$ erreicht, daraufhin ist ein leichtes Absinken des Moduls zu sehen. Es ist im Gegensatz zum vierten Durchlauf kein Ansteigen unterhalb von $600 \mathrm{~K}$ zu beobachten, jedoch wird der Maximalwert bei $720 \mathrm{~K}$ nicht erreicht. Im Verlustmodul ist ein konstanter Verlauf bis $550 \mathrm{~K}$ zu sehen. Der Anfangswert ist etwas größer als beim vierten Durchlauf. Bis $550 \mathrm{~K}$ ist im Gegensatz zur amorphen Probe der vorigen Durchläufe kein Maximum zu erkennen, erst nach $550 \mathrm{~K}$ ist ein sehr breiter Peak (bis 650 K) zu beobachten. Nach dem Maximalwert fällt der Modul wieder ab und läuft ab 660 K parallel zum vierten Durchlauf. 


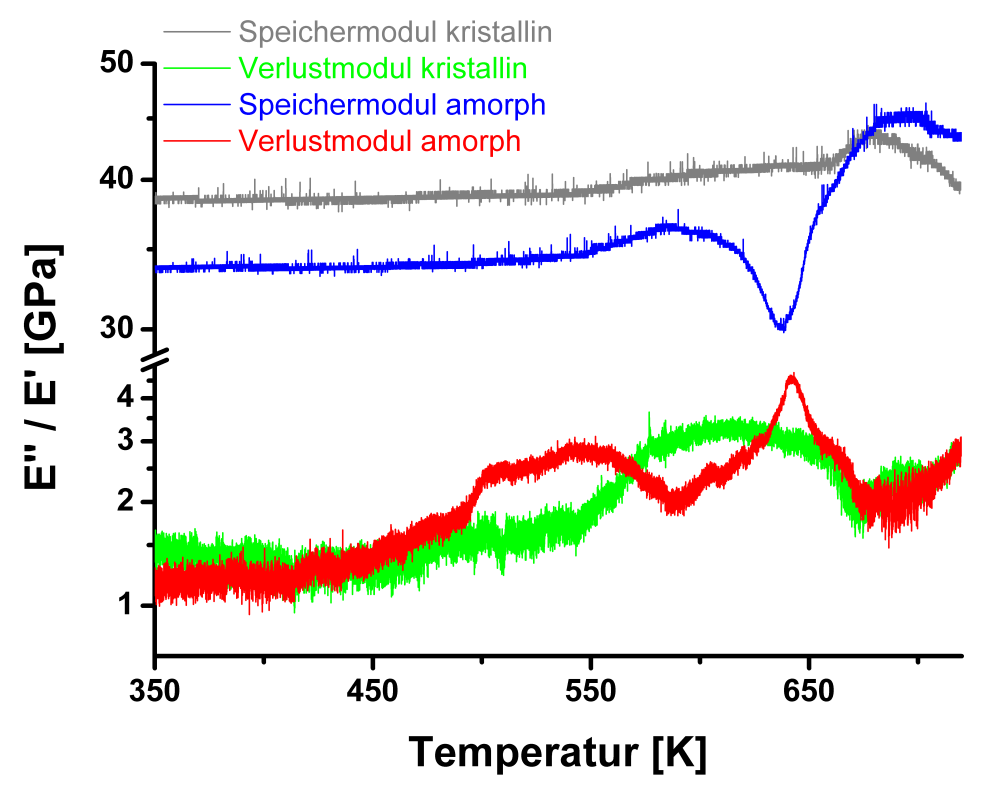

Abbildung 3.4: Gegenüberstellung der Relaxationsvorgänge des vierten Durchlaufs mit der kristallisierten Probe

Im Vergleich zu den mechanischen Messungen an der DMA 7 wurde mit derselben Heizrate von $0,1 \mathrm{~K} / \mathrm{min}$ eine kalorimetrische DSC-Messung vorgenommen, um zu erkennen, ob den Relaxationen unterhalb der Glasübergangstemperatur exotherme oder endotherme Prozesse zugeordnet werden können. Eine endotherme Reaktion kann an einem ansteigenden Kurvenverlauf und eine exotherme an einem abfallenden erkannt werden. Dafür wurde die Probe in $\mathrm{Al}_{2} \mathrm{O}_{3}$ Tiegeln vermessen, wobei der Wärmefluss auf die Probenmasse geeicht und sowohl das kristalline als auch das Signal des leeren Tiegels von der Messung subtrahiert wurden. Die Ergebnisse sind in Abbildung 3.5 dargestellt. Es wurde zunächst bis $600 \mathrm{~K}$ geheizt (schwarze Kurve), danach mit $100 \mathrm{~K} / \mathrm{min}$ bis $50 \mathrm{~K}$ gekühlt und erneut mit $0,1 \mathrm{~K} / \mathrm{min}$ bis $770 \mathrm{~K}$ (rote Kurve) erwärmt. Die blauen Kurven sind eine Glättung von je 5 Datenpunkten zur besseren Übersicht. Beim ersten Durchlauf ist von $350 \mathrm{~K}$ bis $450 \mathrm{~K}$ ein langsamer Anstieg im Wärmefluss zu sehen, gefolgt von einem Knick in der Kurve und einem starken endothermen Verlauf bis hin zu 470 K. Hiernach sinkt der Wärmefluss kurz ab, um mit der Anfangssteigung weiter zu verlaufen. Nach dem Abkühlen ist im zweiten Durchlauf bis $465 \mathrm{~K}$ ein Anstieg mit gleicher Steigung wie beim ersten Durchlauf zu erkennen, wobei der absolute Wärmefluss höher ist. Hierauf folgt wiederum ein endothermer Anstieg bis $477 \mathrm{~K}$, gefolgt von einem kurzen exothermen 


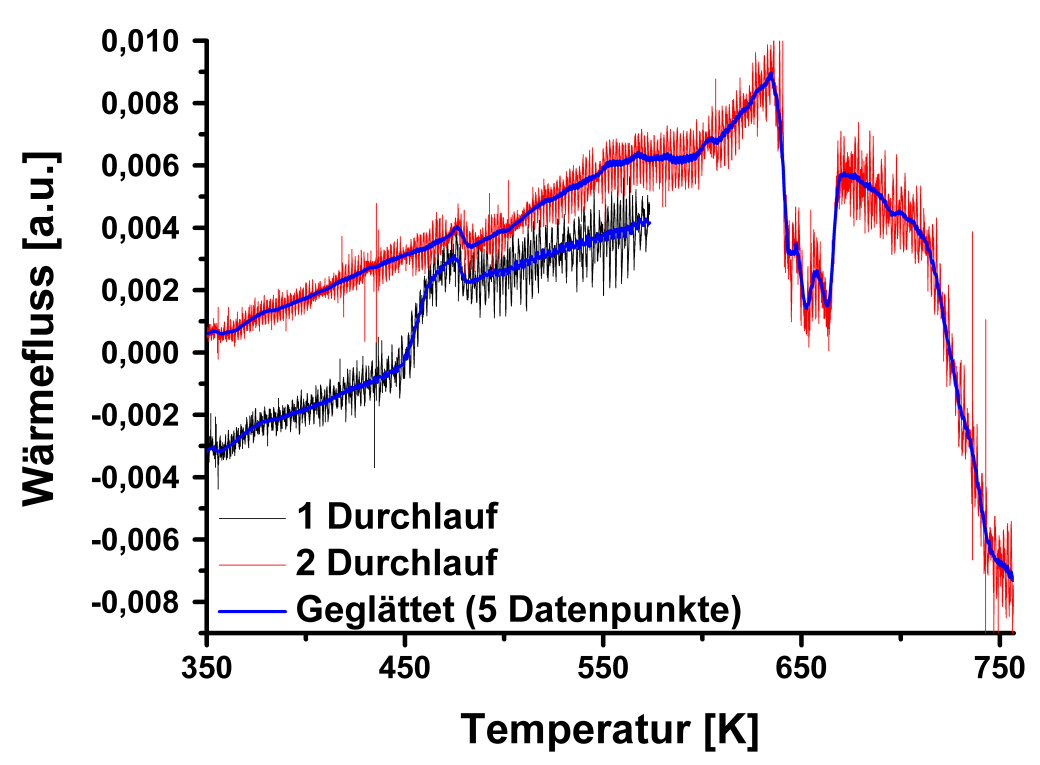

Abbildung 3.5: Vergleich des normierten Wärmeflusses vom ersten (schwarz) und zweiten (rot) Durchlauf bei einer Heizrate von $0,1 \mathrm{~K} / \mathrm{min}$. Die blauen Linien sind über 5 Datenpunkte geglättete Kurven.

Abfall. Danach steigt die Kurve in etwa mit der Anfangssteigung bis ca. $600 \mathrm{~K}$ an. Folgend ist ein Knick in der Kurve zu beobachten, so dass eine größere Steigung bis $635 \mathrm{~K}$ zu sehen ist. Hierauf ist eine starke exotherme Reaktion mit drei Minima (645 K, $652 \mathrm{~K}$, $662 \mathrm{~K})$ zu beobachten, dem ein starker Anstieg des Wärmeflusses (670 K) folgt, wobei eine Differenz von vor zu nach der Kristallisation von $\Delta \mathrm{H}=0,003 \hat{=} 33 \%\left(\left(\Delta \mathrm{E}^{\prime} \hat{=} 28 \%\right)\right.$ aus Abb. 3.2) vorliegt. Bis zum Abschluss der Messung ist dann ein exothermer Verlauf mit einer größer werdenden negativen Steigung bis $745 \mathrm{~K}$ und folgend ein Abflachen der Kurve zu erkennen. Beim Vergleich dieser Messungen mit den mechanischen ist zu bemerken, dass der endotherme Peak (470 K) weit unterhalb der Glasübergangstemperatur im selben Temperaturbereich zu finden ist wie der zusätzlich auftretende Relaxationspeak in den DMA-Messungen (siehe Abb. 3.2). Dies deutet auf eine Reaktion hin, welche nur bei kleinen Heizraten zu sehen ist. Diese These wird im Kapitel 4 genauer diskutiert. Außerdem kann sowohl die Glasübergangstemperatur durch die Steigungsänderung bei $600 \mathrm{~K}$ als auch die Kristallisation an dem starken exothermen Abfall ab $635 \mathrm{~K}$ beobachtet werden, welche ebenfalls mit den Temperaturen und der prozentualen Änderung von E' aus der DMA-Messung im Vergleich zum Wärmefluss H übereinstimmen. 


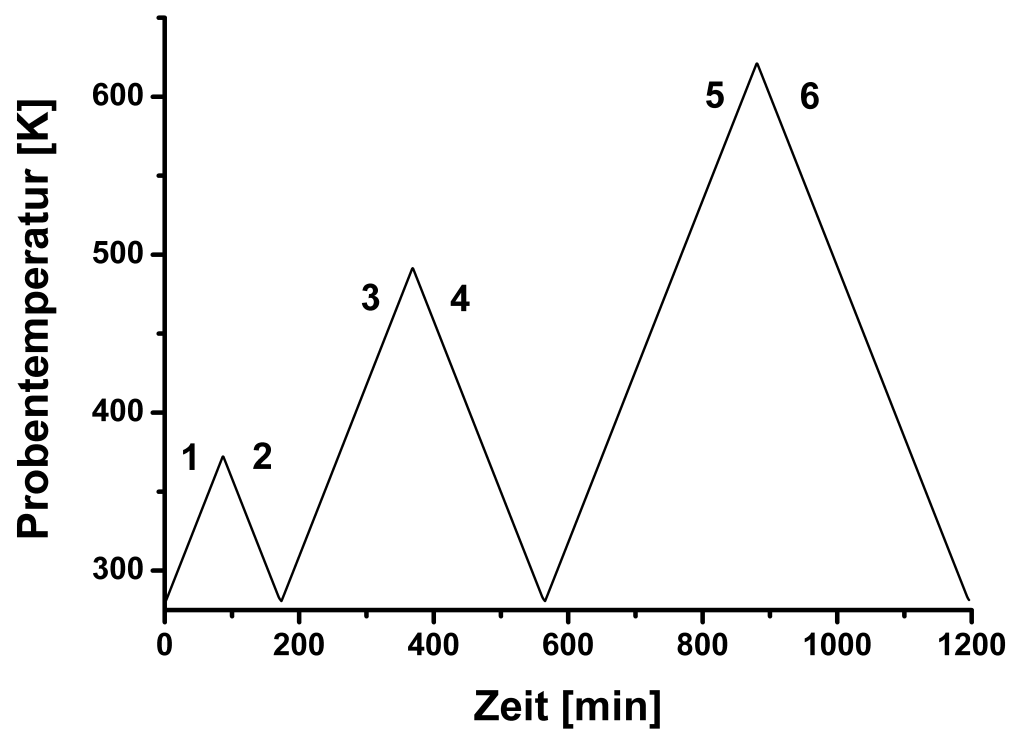

Abbildung 3.6: Temperaturverlauf zur Untersuchung der Elastizitätsmodule bei Erwärmung und Abkühlung der Probe

Relaxationsprozesse unterhalb der Glasübergangstemperatur sind in der Literatur häufig mit $\beta$ - Prozessen in Verbindung gebracht worden, die auch für eine temperaturabhängige Alterung verantwortlich sind (siehe Kapitel 1.4 und 1.4.2). Um diesen Effekt zu untersuchen, wurde mit eine amorphe PdCuSi-Probe $1 \mathrm{~K} / \mathrm{min}$ sowohl erwärmt als auch abgekühlt, wobei während des Versuches die Temperatur nicht die Glasübergangstemperatur überschritt. Die statische Kraft wurde auf $250 \mathrm{mN}$ und die dynamische auf $200 \mathrm{mN}$ eingestellt. In Abbildung 3.6 sind die Temperaturveränderungen, welche für diesen Versuch verwendet wurden, dargestellt. Im ersten Zyklus wurde die Probe von Raumtemperatur auf $370 \mathrm{~K}$ aufgewärmt und anschließend wieder auf die Starttemperatur mit der gleichen Heizrate abgekühlt. In den Schritten 3 und 4 wurde die Probe bis $490 \mathrm{~K}$ erwärmt und ebenfalls wieder auf Raumtemperatur gebracht. Im letzten Zyklus (Schritte 5 und 6) wurde die Probe bis kurz unterhalb der Glasübergangstemperatur bis $620 \mathrm{~K}$ geheizt und dann wieder auf die Anfangstemperatur zurückgekühlt.

Der Verlustmodul, der bei dieser Messung bestimmt wurde, ist in Abbildung 3.7 in Abhängigkeit der Probentemperatur aufgetragen. Der erste Zyklus (Heizschritte 1 und 2) ist in schwarz, der zweite (3/4) in blau und der letzte (5/6) in rot dargestellt. Ebenfalls sind der Start der Messung und im dritten Zyklus die Heizrichtungen mit Pfeilen markiert. 


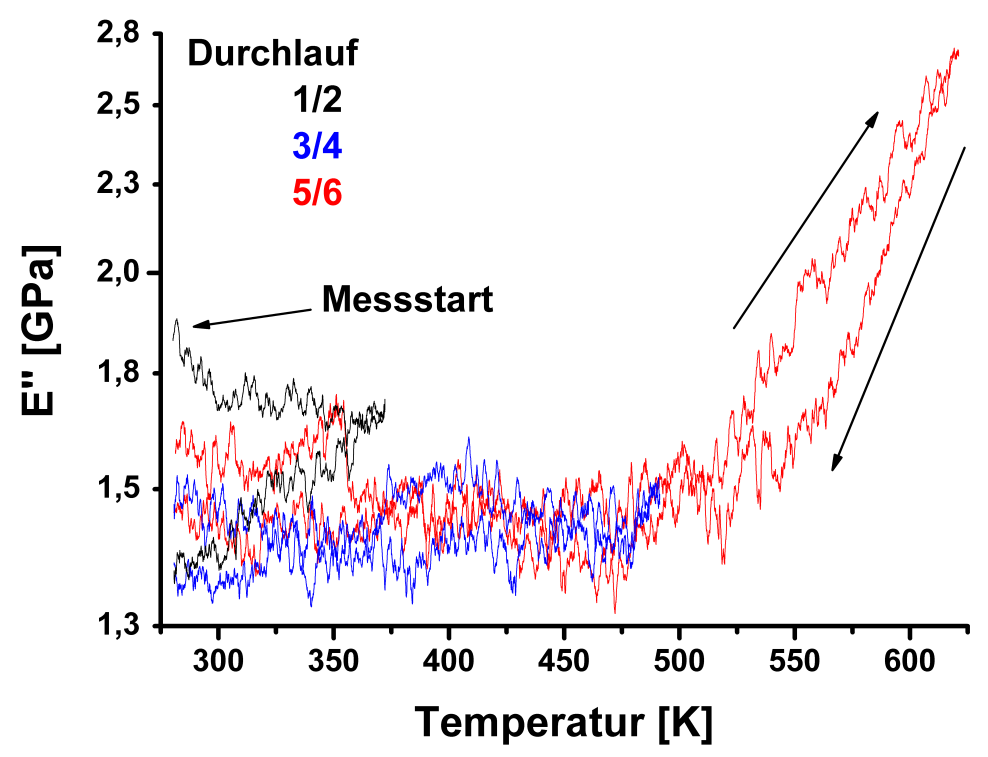

Abbildung 3.7: Verlustmodul beim Erwärmen und Abkühlen der Probe, der erste Zyklus $(1 / 2)$ ist in schwarz, der zweite $(3 / 4)$ in blau und der dritte $(5 / 6)$ in rot dargestellt.

Es ist zu erkennen, dass der Verlustmodul im ersten Zyklus sowohl beim Erwärmen als auch beim Abkühlen zurück auf Raumtemperatur abnimmt. Dabei ist jedoch beim Abkühlen ein stärkerer Effekt zu erkennen. Im zweiten Zyklus ist sowohl beim Heizen als auch beim Abkühlen keine systematische Änderung zu beobachten. Erst im letzten Zyklus ist ein Ansteigen ab $500 \mathrm{~K}$ beim Erwärmen zu sehen. Beim darauffolgenden Schritt 6 nimmt der Modul wieder auf den Anfangswert ab, wobei die Steigung im Temperaturbereich von $620 \mathrm{~K}$ bis $500 \mathrm{~K}$ deutlich steiler und danach konstant ist.

Zum Vergleich ist in Abbildung 3.8 der Speichermodul in Abhängigkeit der Probentemperatur zu sehen. Es wurden alle Zyklen in rot abgebildet, wobei die Heizrichtungen über Pfeile markiert sind. Es ist ein Startmodul bei Raumtemperatur von 34,5 GPa zu erkennen, welches im ersten Heizschritt konstant bleibt. Beim Abkühlen im ersten Zyklus ist ein Ansteigen im Modul zu erkennen, wobei bei Raumtemperatur ein Modul von $36 \mathrm{GPa}$ erreicht wird. Im zweiten Zyklus sinkt der Modul zuerst beim Erwärmen bis $400 \mathrm{~K}$ ab und steigt anschließend auf etwa denselben Wert wieder an. Beim Zurückkühlen auf Raumtemperatur ist weiterhin ein steigender Modul zu erkennen, wobei am Ende dieses Schrittes ein Wert von 38,5 GPa erreicht wird. Im dritten Zyklus ist im 5. Schritt 


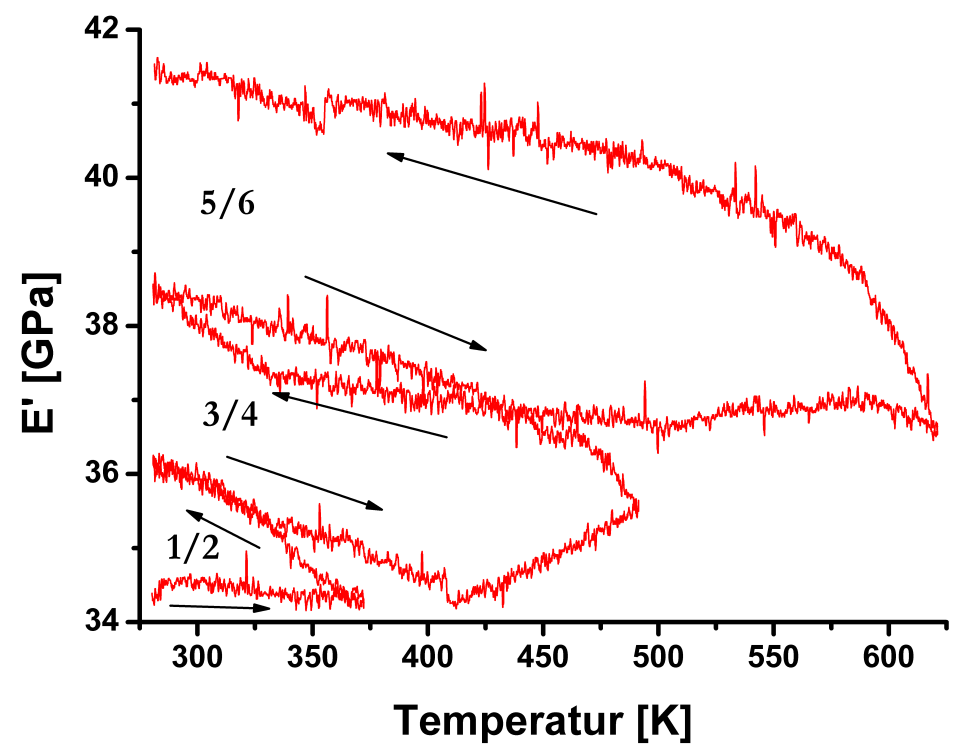

Abbildung 3.8: Speichermodul beim Erwärmung und Abkühlung der Probe

zunächst ein fallender Modul zu sehen, wobei dieser ab 450 K mit 36,8 GPa annähernd konstant verläuft. Beim letzten Schritt steigt der Modul bis 500 K stark an (40 GPa). Beim weiteren Abkühlen ist ein annähernd linearer Anstieg auf 41,5 GPa zu erkennen. Insgesamt hat sich somit der Modul um $7 \mathrm{GPa}$ von 34,5 GPa auf 41,5 GPa erhöht, wobei die größten Änderungen beim Abkühlen beobachtet wurden. 


\subsubsection{Untersuchung von Spannungs-Dehnungskurven}

Spannungs-Dehnungskurven bieten die Möglichkeit, den Übergang vom elastischen Verhalten eines Materials zum inelastischen bis hin zum viskosen Fließen zu beobachten. Dabei können verschiedene materialspezifische Größen wie z. B. der Elastizitätsmodul bestimmt werden (siehe Kapitel 1.3.1.2). Außerdem bietet diese Analysemethode die Aussicht, das Materialverhalten im Sinne der PEL (siehe Kapitel 1.4.1) diskutieren zu können und neue Einsichten in die Energielandschaft zu erhalten. In diesem Kapitel werden nun die Ergebnisse der Untersuchung von Spannungs-Dehnungskurven am amorphen PdCuSi vorgestellt. Dabei wurde sowohl das Verhalten bei verschiedenen Temperaturen als auch bei unterschiedlichen Kraftänderungen beobachtet.

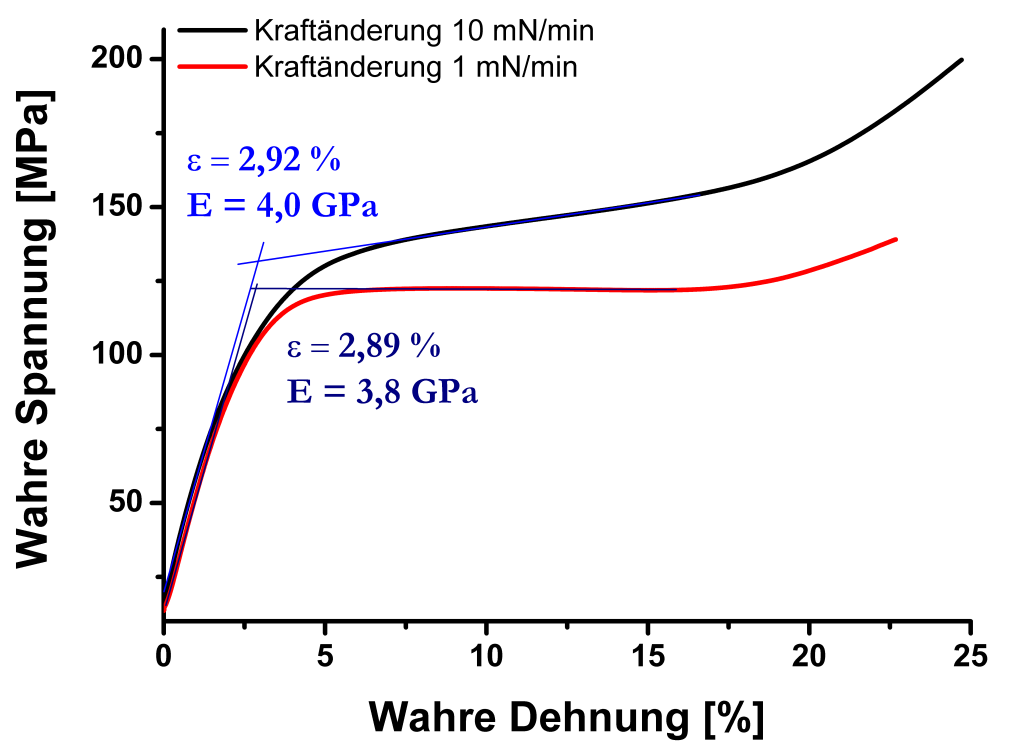

Abbildung 3.9: Spannungs-Dehnungskurven von Haaren bei einer Kraftänderung von $10 \mathrm{mN} / \mathrm{min}$ (schwarz) und $1 \mathrm{mN} / \mathrm{min}$ (rot). In blau sind die Verlängerungen der linearen Bereiche eingezeichnet

Um die gerätespezifischen Parameter der verwendeten DMA 7 einschätzen zu können, wurden als Vorversuche menschliche Haare (polymeres, amorphes Gemisch mit kristallinen Anteilen) bei Raumtemperatur mit zwei unterschiedlichen Kraftänderungen untersucht. In Abbildung 3.9 sind die erhaltenen Kurven dargestellt. In schwarz ist die Kurve aufgenommen mit einer Kraftänderung von $10 \mathrm{mN} / \mathrm{min}$ und in rot mit $1 \mathrm{mN} / \mathrm{min}$ zu sehen. Die blauen Geraden sind eine Verlängerung des linearen Anfangsbereiches bzw. 
des viskosen Fließens, um die Yielding Punkte bestimmen zu können.

$\mathrm{Zu}$ erkennen ist, dass mit zunehmender Spannung die Proben zunächst proportional zu dieser gedehnt werden. Dabei ist für die größere Kraftänderung zu beobachten, dass der elastische, lineare Anteil geringer ist und die Kurve sich früher als bei der kleineren Kraftänderung zu krümmen beginnt. Nach dem Erreichen des Yielding Points (bestimmt für $10 \mathrm{mN} / \mathrm{min}$ bei einer Dehnung von 2,92\% und bei $1 \mathrm{mN} / \mathrm{min}$ bei 2,89\%), zeigen die Proben ein viskoses Fließen, wobei in der schwarzen Kurve ein Ansteigen, bedingt durch die schnelle Kraftänderung, zu beobachten ist. Eine weitere Krümmung, die ein steiferes Verhalten des Materials anzeigt, ist bei der schwarzen Kurve nach 17,6 \% Dehnung und bei der roten nach 17,0 \% zu erkennen. Hervorgerufen wird dies durch ein paralleles Ausrichten und eine maximale Streckung der Polymerketten in den Haaren. ${ }^{[20]}$ Desweiteren ist der Bruch der Proben bei 24,8 \% Dehnung $(10 \mathrm{mN} / \mathrm{min}$ Kraftänderung) und 22,7 \% (Kraftänderung $1 \mathrm{mN} / \mathrm{min}$ ) zu sehen. Der größere Elastizitätsmodul konnte bei der höheren Kraftänderung bestimmt werden (4,0 GPa zu $3,8 \mathrm{GPa})$.

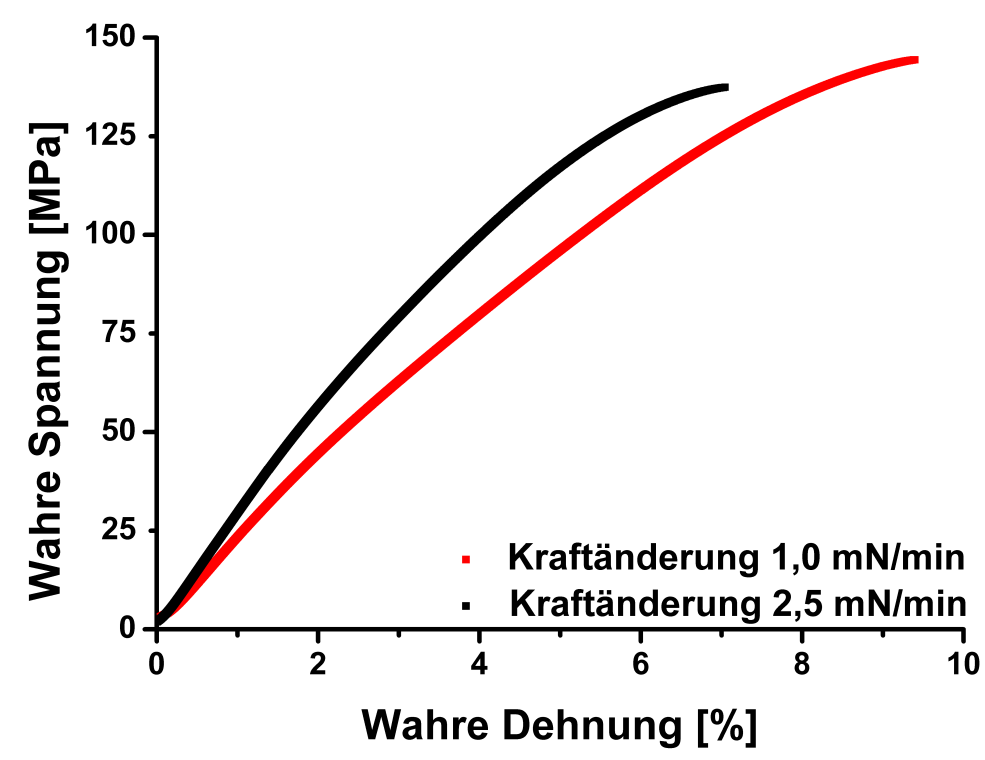

Abbildung 3.10: Spannungs-Dehnungskurven von PdCuSi bei $640 \mathrm{~K}$ mit zwei unterschiedlichen Kraftänderungen

Ein vergleichbarer Versuch am metallischen Glas PdCuSi ist in Abbildung 3.10 zu sehen. Es wurden zwei extrem dünne Proben (Breite ca. 0,1 mm / Dicke ca. 0,02 mm ) bei $640 \mathrm{~K}$ 
mit zwei unterschiedlichen Kraftänderungen untersucht $(1,0 \mathrm{mN} / \mathrm{min}$ - rote Kurve bzw. 2,5 mN/min - schwarze Kurve). Es ist zu erkennen, dass kleinere Dehnungen bis zum Bruch der Proben (7,0 \% bzw. 9,5 \% Dehnung) als bei den Haarproben erreicht werden, ohne in den Bereich des viskosen Fließens zu gelangen. Deshalb konnten bei diesem Versuch keine Yielding Punkte bestimmt werden. Es wurde jedoch bei der kleineren Kraftänderung eine flachere Steigung im elastischen Anteil beobachtet. Wie bei den Haarproben ist somit der Elastizitätsmodul mit 4,1 GPa bei einer Kraftänderung von $1 \mathrm{mN} / \mathrm{min}$ kleiner als bei der größeren Kraftänderung ( $\mathrm{E}=5,2 \mathrm{GPa}$ bei $2,5 \mathrm{mN} / \mathrm{min}$ ).

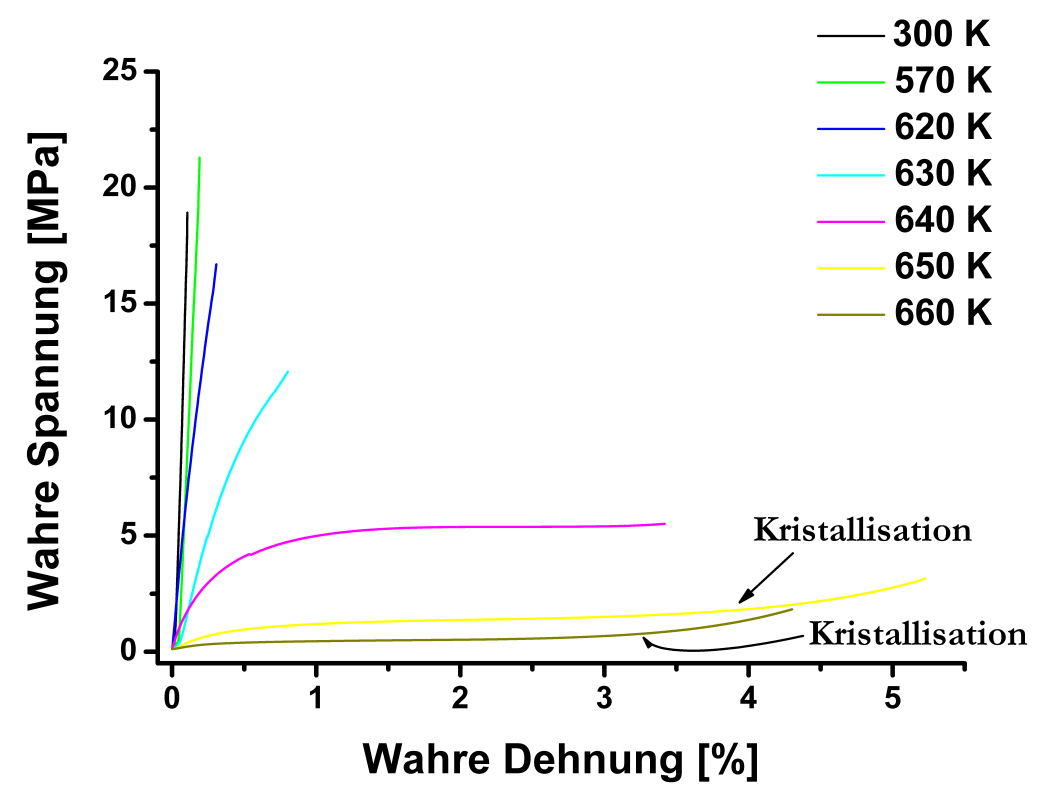

Abbildung 3.11: Vergleich der Spannungs-Dehnungskurven von PdCuSi mit einer Kraftänderung von $5 \mathrm{mN} / \mathrm{min}$ bei verschiedenen Temperaturen

Im Vergleich zu diesem Versuch sind in Abbildung 3.11 die Änderungen des Dehnungsverhaltens in Abhängigkeit von der Temperatur dargestellt. Es wurde die Kraftänderung mit $5 \mathrm{mN} / \mathrm{min}$ konstant gehalten und die Temperatur von Raumtemperatur bis über die Glasübergangstemperatur erhöht. Dabei wurde zu jeder Temperatur die Dehnung in Abhängigkeit von der Kraftänderung aufgenommen. Es ist zu bemerken, dass die Proben (Abmaße wie in Kapitel 2.1 beschrieben) bei keiner Messung gerissen sind, sondern die Messungen durch Erreichen der maximalen Kraft beendet wurden.

$\mathrm{Zu}$ erkennen ist, dass die Steigungen im elastischen Teil der Kurven mit Erhöhung der Temperatur flacher werden. Bei $300 \mathrm{~K}$ und $570 \mathrm{~K}$ ist keine große Veränderung in den 
Kurven zu sehen. Erst ab $620 \mathrm{~K}$ ist ein leichtes Abknicken zu erkennen, jedoch reicht die Kraft nicht aus, um komplett in den Bereich des viskosen Fließens zu gelangen. Bei $630 \mathrm{~K}$ ist ein klarer Übergang in den nichtlinearen Bereich zu beobachten, so dass eine deutlich größere Dehnung (0,8 \%), aber kein viskoses Fließen erreicht wird. Erst bei Erhöhung der Temperatur auf $640 \mathrm{~K}$ kann sowohl ein Yielding Point bestimmt $\left(\epsilon_{c}=0,4 \%\right)$ als auch ein viskoses Fließen beobachtet werden. Es wird eine maximale Dehnung von 3,42 \% erreicht. Dieser Wert ist nur begrenzt durch die maschinelle Beendigung des Versuches. Bei Temperaturen oberhalb der Glasübergangstemperatur ist der lineare Anteil der Kurve kaum mehr zu erkennen. Bei $650 \mathrm{~K}$ lässt sich der Yielding Point bei 0,30 \% und bei $660 \mathrm{~K}$ bei 0,28 \% Dehnung bestimmen. Dabei ist das viskose Fließen stark ausgeprägt, so dass Dehnungen von über $4 \%$ erreicht werden, bevor die maximale Kraft erreicht und der Versuch beendet wird. Es ist jedoch ein deutliches Abknicken der Kurven (nach $4 \%$ Dehnung bei $650 \mathrm{~K}$ und nach 3,2 \% Dehnung bei $660 \mathrm{~K}$ ) zu sehen. Hier beginnt offentsichtlich die Kristallisation der Gläser, wodurch das Material steifer wird. Diese Punkte sind in der Abbildung mit Kristallisation und schwarzen Pfeilen markiert.

a)

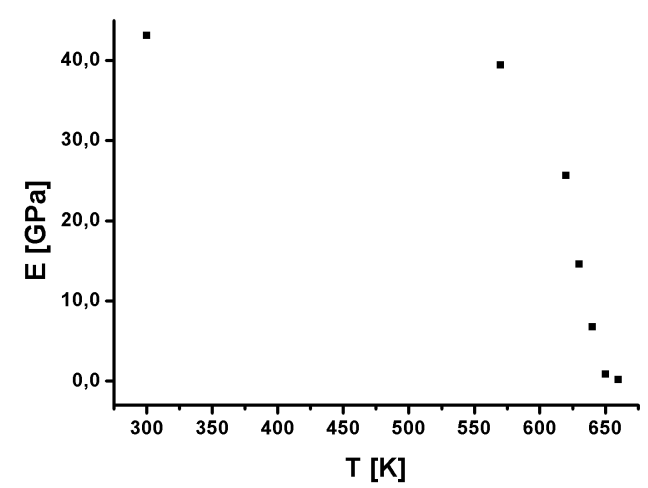

b)

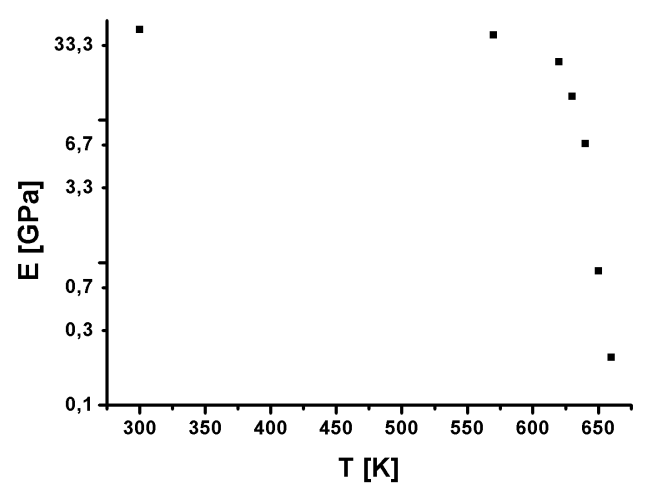

Abbildung 3.12: Bestimmung der Elastizitätsmodule der Spannungs-Dehnungskurven aus Abb. 3.11 in linearer Auftragung im Bildteil a) und logarithmischer im Bildteil b).

Aus den elastischen, linearen Anteilen der Kurven wurden die Elastizitätsmodule bestimmt, welche in Abbildung 3.12 sowohl in linearer als auch in halblogarithmischer Auftragung $\mathrm{zu}$ sehen sind. Bei Raumtemperatur konnte ein Modul von $43 \mathrm{GPa}$ berechnet werden. Bei höheren Temperaturen nimmt dieser Wert deutlich ab (570 K - 39 GPa, 620 K - 25 GPa) und bricht beim Erreichen der Glasübergangs- 
temperatur ein $(630 \mathrm{~K}$ - $14 \mathrm{GPa}, 640 \mathrm{~K}-7 \mathrm{GPa})$. Oberhalb von $\mathrm{T}_{G}$ sind nur noch Elastizitätsmodule von 0,9 GPa $(650 \mathrm{~K})$ und 0,2 GPa $(660 \mathrm{~K})$ zu bestimmen.

a)

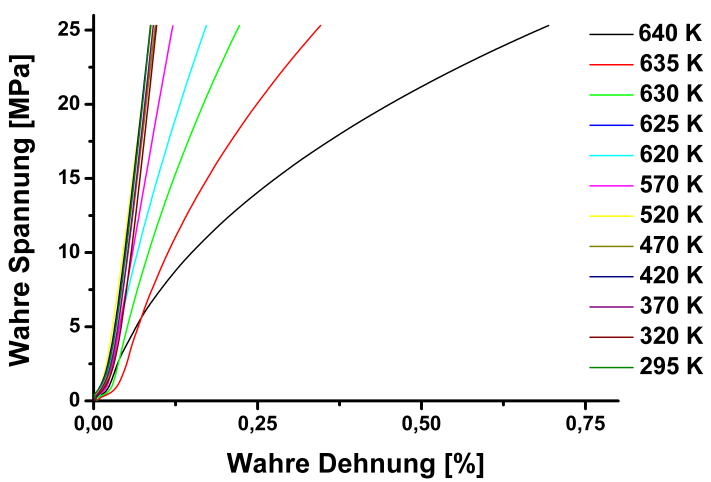

b)

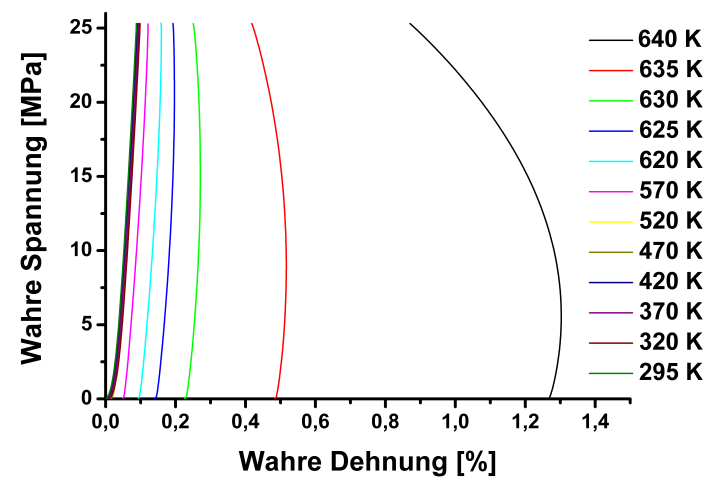

Abbildung 3.13: Vergleich der Spannungs-Dehnungskurven von PdCuSi mit einer Kraftänderung von a) $300 \mathrm{mN} / \mathrm{min}$ und b) $-300 \mathrm{mN} / \mathrm{min}$

Um ein genaueres Bild des Elastizitätsverhaltens zu erhalten, wurden SpannungsDehnungskurven im Temperaturbereich von Raumtemperatur bis $640 \mathrm{~K}$ mit einem Temperaturabstand von $50 \mathrm{~K}$ (bis $620 \mathrm{~K}$ ) bzw. $5 \mathrm{~K}(620 \mathrm{~K}$ bis $640 \mathrm{~K}$ ) aufgenommen. Dabei wurden die Versuche mit einer Kraftänderung von 300 mN/min durchgeführt. Nachdem die maximale Kraft bei einer Probe erreicht wurde, wurde diese anschließend mit derselben Kraftänderung wieder zurückgefahren. Die so erhaltenen Kurven sind in Abbildung $3.13 \mathrm{zu}$ sehen, wobei im Bildteil a) die Kurven mit positiver Kraftänderung und im Teil b) die mit negativer Kraftänderung dargestellt sind. Klar zu erkennen ist, dass im Temperaturbereich bis $570 \mathrm{~K}$ der lineare Bereich der Spannungs-Dehnungskurven nicht verlassen wird. Erst beim Erreichen höherer Temperaturen sind Krümmungen zu sehen, wobei diese mit steigender Temperatur stark zunehmen. Sowohl im Bildteil a) als auch b) sind bei Temperaturen weit unterhalb von $\mathrm{T}_{G}$ keine Unterschiede zu beobachten. Bei positiver Krafterhöhung ist das temperaturabhängige Erweichen des Materials beim Erreichen der Glasübergangstemperatur über die zunehmende Krümmung zu erkennen. Trotzdem kann in allen Kurven ein linearer Bereich beobachtet werden, welcher der Bestimmung der Elastizitätsmodule dient. Im Bildteil b) fällt auf, dass die Proben bei Temperaturen in der Nähe von $\mathrm{T}_{G}$ trotz negativer Kraftänderung weiterhin länger werden. Das Erreichen des linearen Teils ist jedoch bei derselben Spannung zu beobachten wie der Beginn der Krümmungen im Bildteil a). Je größer die eingestellte Temperatur 
ist, desto kleiner ist die maximale Spannung, die zum elastischen Teil beiträgt. Auch bei negativer Kraftänderung kann somit ein linearer Teil der Kurven verwendet werden, um die Elastizitätsmodule zu errechnen. Diese können mit denen aus den Messungen mit positiver Kraftänderung verglichen werden.

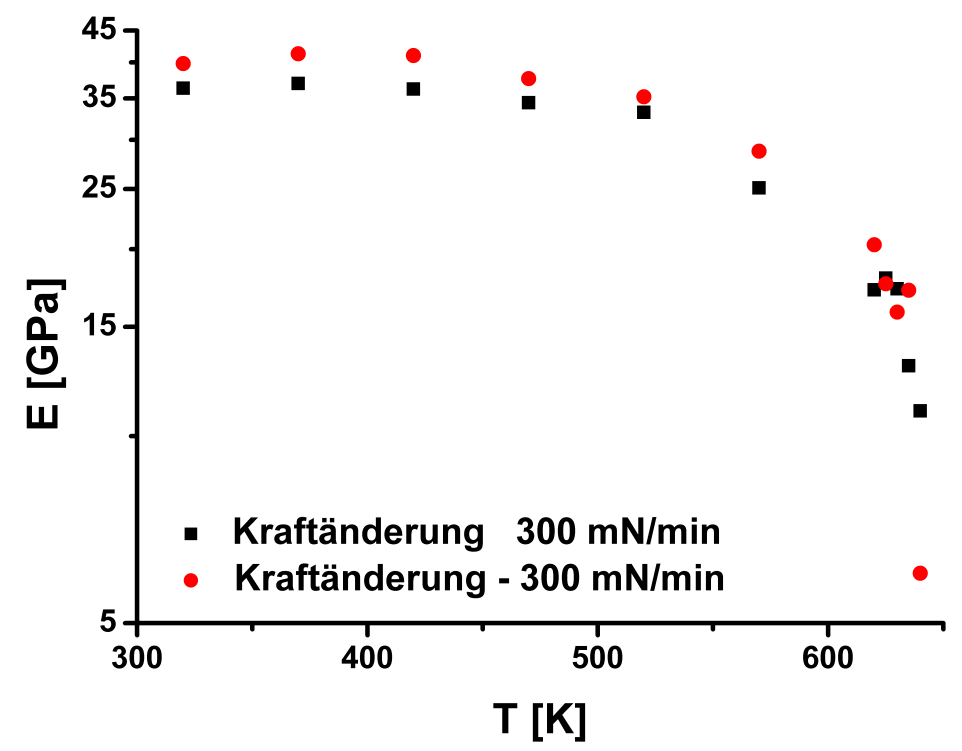

Abbildung 3.14: Bestimmung der Elastizitätsmodule der Spannungs-Dehnungskurven aus Abb. 3.11, die schwarzen Punkte sind die Werte zugehörig zur positiven, die roten zur negativen Kraftänderung

Die bestimmten Module sind in Abbildung $3.14 \mathrm{zu}$ sehen. In schwarz sind die Werte für die positive und in rot für die negative Kraftänderung dargestellt. Unterhalb von $\mathrm{T}_{G}$, im Bereich von Raumtemperatur bis zu $600 \mathrm{~K}$, liegen die Werte für die negative Kraftänderung über denen der Messung mit positiver. Erst bei Temperaturen oberhalb von $600 \mathrm{~K}$ sind wegen der größeren Messfehler und durch den sehr kleinen linearen Bereich darüber keine qualitativen Aussagen mehr zu machen. Bei Raumtemperatur wurde für die positive Kraftänderung ein Anfangsmodul von 37 GPa und beim Zurückfahren der Kraft von 40 GPa bestimmt. Diese Werte sind bis $420 \mathrm{~K}$ konstant und nehmen mit steigender Temperatur ab. Bei $600 \mathrm{~K}$ wurde nur noch ein Modul von 17 GPa (schwarz) und $20 \mathrm{GPa}$ (rot) bestimmt, womit die Differenz zwischen den Werten immer noch der Anfangsdifferenz entspricht. Der niedrigste Modul wurde bei zurückgehender Kraft bei $640 \mathrm{~K}$ mit 6 GPa errechnet. 


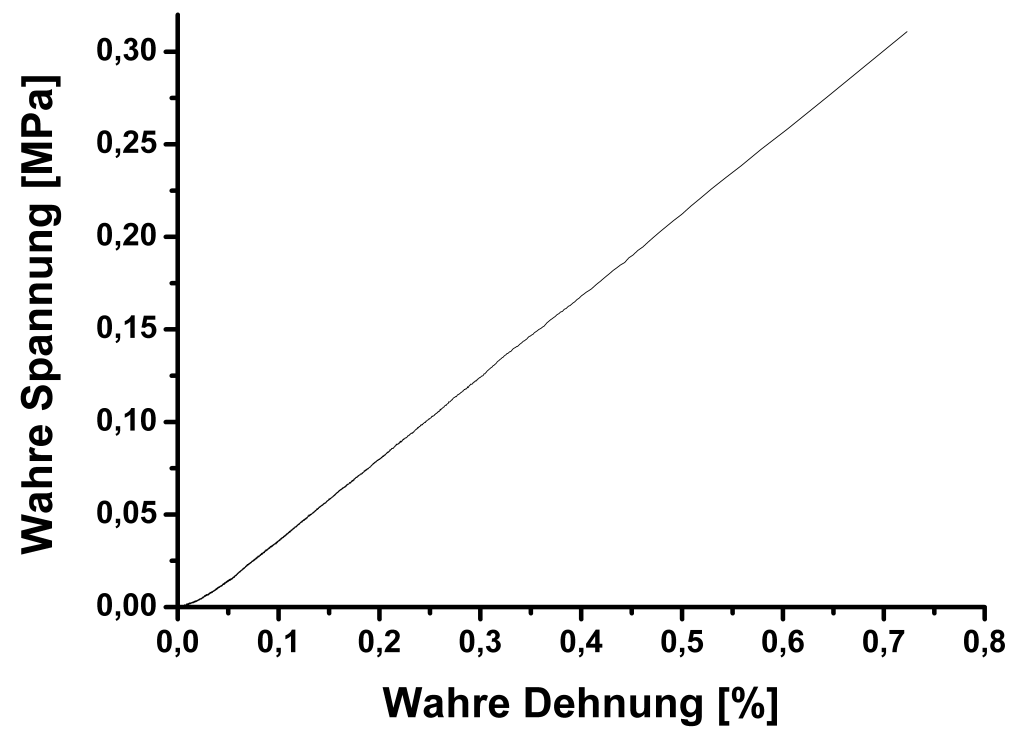

Abbildung 3.15: Spannungs-Dehnungskurve von PdCuSi bei Raumtemperatur mit einer Dehnungsrate von $1 \cdot 10^{-7} \mathrm{~s}^{-1}$

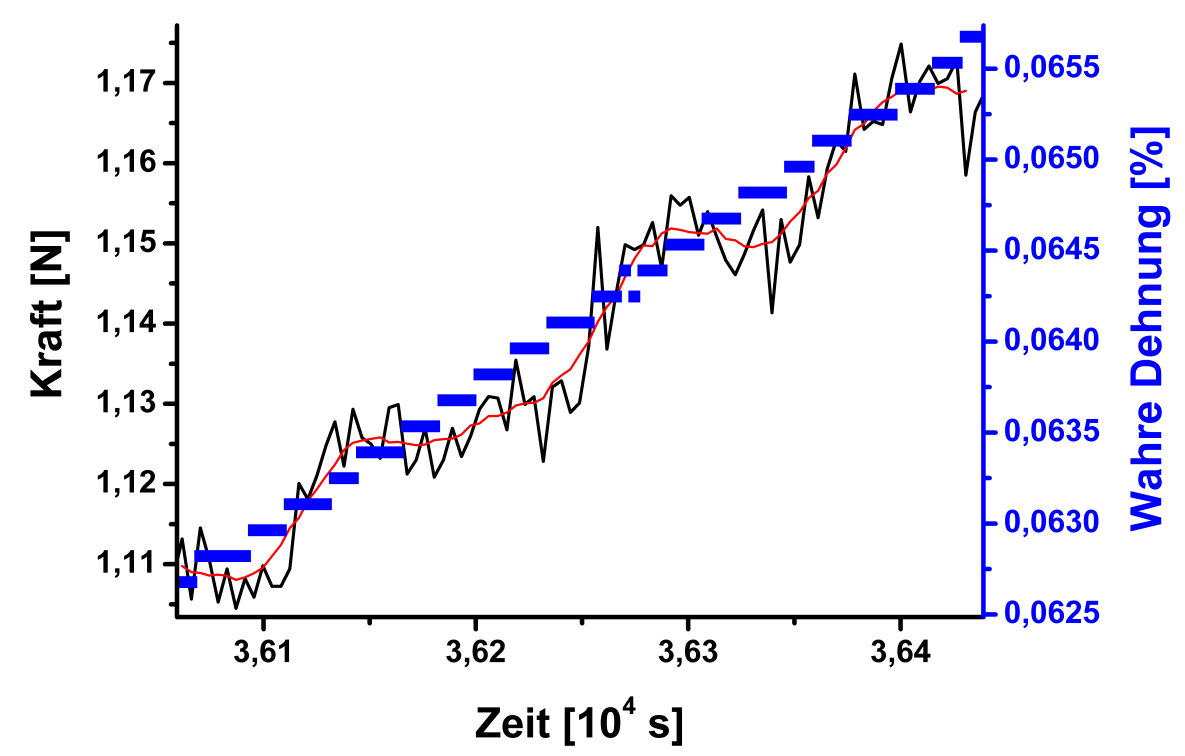

Abbildung 3.16: Vergleich der Probendehnung (blau) mit der aufgenommenen Kraftänderung (schwarz). Die rote Linie zeigt eine Glättung der Kraft über 10 Datenpunkte. 
Im Vergleich zu den spannungskontrollierten Versuchen mit Hilfe der DMA 7 wurden ebenfalls Messungen mit einer Zwicki durchgeführt (siehe Kapitel 2.1.3). Hierbei konnten die Messungen dehnungskontrolliert, aber nur bei Raumtemperatur durchgeführt werden. Es wurde eine Dehnungsrate von $1 \cdot 10^{-7} \mathrm{~s}^{-1}$ gewählt, wobei alle $2 \mathrm{~s}$ ein Datenpunkt aufgenommen wurde.

In Abbildung 3.15 ist eine Messung an einer amorphen PdCuSi-Probe zu sehen. Es wurde nur eine Dehnung von 0,7 \% erreicht, bis die Probe an der Einspannung gerissen ist. Der lineare Bereich konnte trotz der sehr kleinen Dehnungsrate nicht verlassen werden. Beim Betrachten eines Ausschnittes der Messung konnte jedoch sowohl das Dehnungsverhalten als auch die gemessene Kraftänderung genauer untersucht werden. In Abbildung 3.16 ist ein solches Bild mit Dehnung (blau) im Vergleich zur aufgenommenen Kraft (schwarz) zu sehen. In der Dehnung können Stufen beobachtet werden, die im Abstand von etwa 20 s auftreten und durch einen internen Regelparameter hervorgerufen werden.

In der dazugehörigen Kraft hingegen sind Sprünge zu erkennen. Obwohl eine Dehnung gehalten wird, ist die Kraft nicht konstant, sondern zeigt ein unregelmäßiges Verhalten, was auf eine nicht methodische Ursache hindeutet. Sowohl die Größe der einzelnen Sprünge als auch die Zeitabstände und die Richtung verändern sich und zeigen kein wiederkehrendes Muster. Innerhalb der Kraftsprünge lässt sich eine übergeordnete Schwingung mit einem Zeitabstand von etwa 100 s erkennen, welche zur einfacheren Darstellung als rote Linie (geglättete Kurve über 10 Datenpunkte) in die Messung eingezeichnet wurde. 


\subsubsection{Untersuchung von Creep/Recoverykurven}

Um die rheologischen Eigenschaften von amorphen Metallen weiter zu untersuchen, wurde aufbauend auf den Ergebnissen der Spannungs-Dehnungskurven (siehe Kapitel 3.1.2) das Kriechverhalten der Proben beobachtet. Dazu wurden Creep/Recoverymessungen am amorphen Material im Vergleich zu kristallisierten Proben aufgenommen. Hierzu wurde wie im Kapitel 2.1.4 beschrieben das Nachkriechen des Materials bei Kraftsprüngen mit zwei unterschiedlichen Kräften beobachtet.

a)

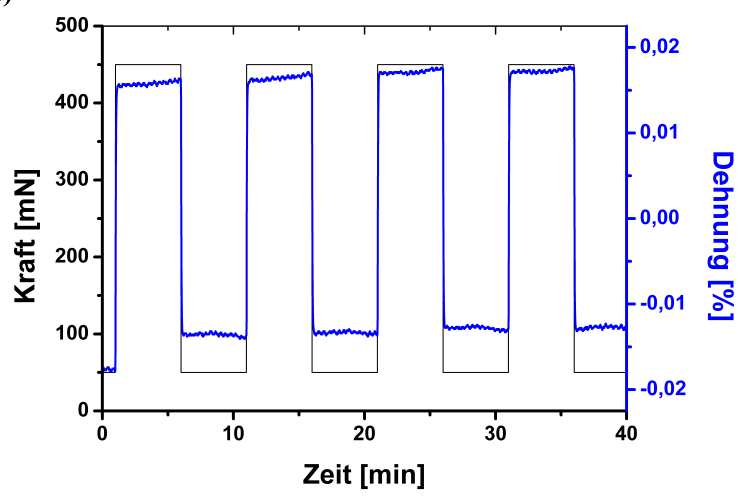

b)

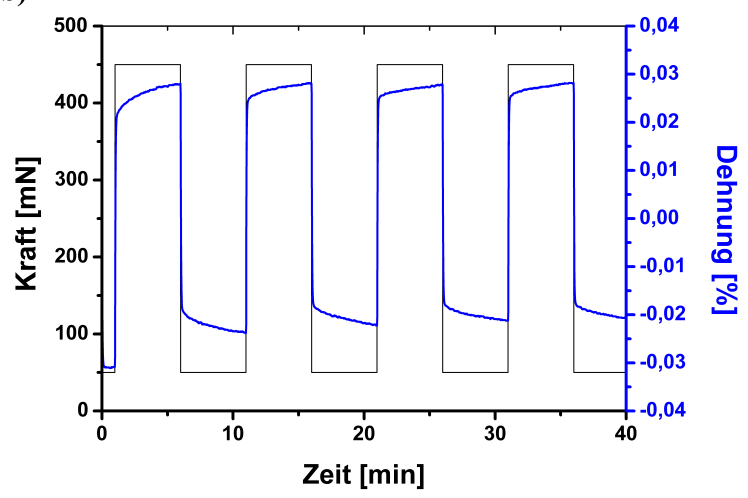

Abbildung 3.17: Creep/Recoverykurven von a) einer kristallisierten und b) einer amorphen PdCuSi Probe bei 570 K. Die Kräfte betrugen $450 \mathrm{mN}$ und $50 \mathrm{mN}$. $\mathrm{Zu}$ sehen ist die Probendehnung (blau) bzw. die verwendeten Kräfte (schwarz) gegenüber der Versuchszeit bei vier Wiederholungen der Kraftsprünge.

In Abbildung 3.17 sind zwei Beispielmessungen zu sehen. Es ist eine kristallisierte (a) im Vergleich mit einer amorphen (b) PdCuSi Probe für vier Kraftwiederholungen dargestellt. In schwarz ist die angelegte Kraft dargestellt, welche jeweils nach 5 Minuten sprunghaft geändert wird, wobei als maximale Kraft $450 \mathrm{mN}$ (entspricht $10 \mathrm{MPa}$ ) und als minimale $50 \mathrm{mN}$ (entspricht $1 \mathrm{MPa}$ ) gewählt wurde. In blau ist demgegenüber das Materialverhalten zu erkennen, welches als Probendehnung aufgetragen wurde. Die dargestellten Versuche wurden bei $570 \mathrm{~K}$ durchgeführt und die Kraft und die Dehnung gegenüber der Zeit mit einem Datenpunkt pro Sekunde aufgezeichnet. Die Dehnung wurde bei der mittleren Anfangskraft vor Beginn des Versuches $(250 \mathrm{mN})$ als $0 \%$ definiert, so dass eine positive Dehnung beim Kraftsprung zur maximalen Kraft und eine negative beim Zurückspringen zu beobachten ist. Das Materialverhalten der kristallisierten Probe 
zeigt nur kleine Dehnungsänderungen bei den Kraftsprüngen von maximal 0,033 \%, beim ersten Kraftsprung und folgend konstant 0,03 \%. Das Material reagiert rein elastisch auf die Änderung der Kräfte, so dass nur ein geringes Nachkriechen, vor allem durch innere Reibungen der DMA bedingt, zu erkennen ist. Es wird eine Delay-time von 8 s - 13 s beobachtet. Die gemittelte Delay-time für die Kraftsprünge zur oberen als auch zur unteren Kraft beträgt 9,975 s (folgend Delay-time x-tal genannt) und wurde zur Normierung der ermittelten Delay-time verwendet.

Bei der amorphen PdCuSi Probe sind im Vergleich zur kristallinen Probe deutlich größere Dehnungen zu beobachten. Bei der ersten Kraftänderung ist eine Dehnung von 0,058 \% zu sehen, welche fast doppelt so groß wie bei der kristallinen Probe ist. Folgend wird noch eine Dehnung bei den Kraftsprüngen von 0,05 \% erreicht. Ebenfalls ist ein deutliches Nachkriechen mit einer zum Kristall größeren Delay-time zu sehen, welche gemittelt für die maximale Kraft bei 147,8 s und für die kleinere Kraft bei 99,9 s lag.

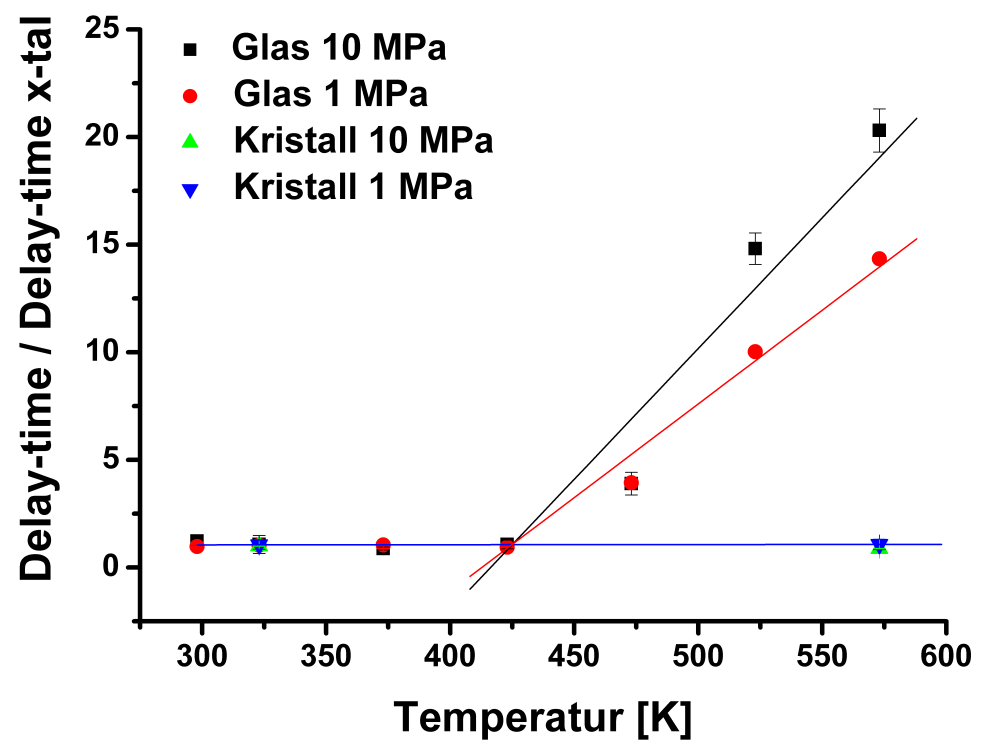

Abbildung 3.18: Delay-time vom amorphen PdCuSi im Vergleich zu kristallisierten Proben

Um eine systematische Untersuchung des Kriechverhaltens vorzunehmen, wurden Creep/Recoverymessungen von Raumtemperatur bis $570 \mathrm{~K}$ vom amorphen PdCuSi im Temperaturabstand von $50 \mathrm{~K}$ aufgenommen. Dabei wurden die erhaltenen Nachkriechzeiten auf die der kristallinen Proben normiert, um alle gerätespezifischen Dämpfungen 
herauszufiltern. Die erhaltenen Delay-Zeiten sind in Abbildung 3.18 dargestellt.

Es wurde bei allen Versuchen eine untere Kraft von $50 \mathrm{mN}$ (entspricht einer Spannung von $1 \mathrm{MPa}$ ) und eine obere von $450 \mathrm{mN}$ (entspricht $10 \mathrm{MPa}$ ) eingestellt. Die erhaltenen Daten sind für die untere Kraft als rote Punkte und für die obere als schwarze Quadrate abgebildet. Zum Vergleich wurde ebenfalls bei 325 K und 570 K eine kristalline Probe (untere Kraft blaue Dreiecke, obere Kraft grüne) gemessen. Die Fehler wurden über die Standardabweichung der 6 Kraftwiederholungen berechnet. Es ist zu erkennen, dass in der Delay-time keine temperatur- oder kraftabhängigen Änderungen bei den kristallinen Proben zu bestimmen sind. Das konstante Verhalten ist in Abbildung 3.18 als blaue Linie dargestellt.

Bei den amorphen Proben konnte bis $425 \mathrm{~K}$ kein Unterschied zu den kristallinen Proben beobachtet werden. Erst bei höheren Temperaturen war eine Veränderung in der Delaytime zu sehen. Sowohl für die obere als auch die untere Kraft steigt die Dämpfung und somit die Delay-time an und ist erheblich größer als bei den kristallinen Proben. Dabei ist ein spannungsabhängiges Verhalten zu erkennen. Bei Temperaturen oberhalb von 475 K liegt die Delay-time der oberen Kraft über der der kleineren. Dieses Verhalten wurde in der Abbildung als linear angenommen und ist als Linie in den Farben der einzelnen Kräfte abgebildet. Dabei ist zu erkennen, dass sich die Ausgleichsgeraden bei der normierten Delay-time des Kristalls bei $425 \mathrm{~K}$ schneiden.

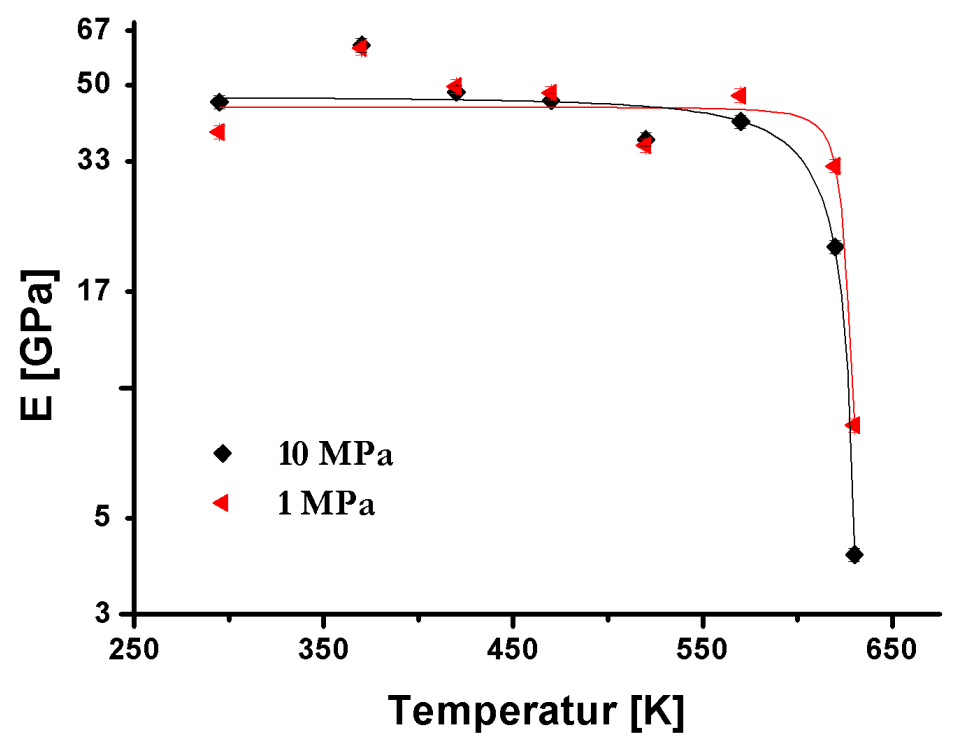

Abbildung 3.19: Berechneter Elastizitätsmodul aus den Creep/Recoverymessungen 
Aus den linearen Anfangsbereichen der Creep/Recoverymessungen direkt nach dem Kraftsprung wurde der Elastizitätsmodul nach Gleichung 1.9 bestimmt und gegenüber der Temperatur aufgetragen (Abb. 3.19). Die Werte für die größere Spannung sind wie in Abbildung 3.18 in schwarz und die für die kleinere in rot aufgetragen. Das Verhalten wurde über Lorentz-fits abgebildet. ${ }^{1}$ Es ist zu erkennen, dass bei Temperaturen weit unterhalb von $\mathrm{T}_{G}$ kein signifikanter Unterschied mit den verwendeten Kräften zu bestimmen ist und ein Elastizitätsmodul von $40 \mathrm{GPa}$ erhalten wird. Bei Temperaturen nahe $\mathrm{T}_{G}$ erscheint der Elastizitätsmodul kraftabhängig zu werden, so dass kleinere Werte für die größere Kraft bestimmt werden und sich $\mathrm{T}_{G}$ zu niedrigeren Temperaturen verschiebt. Dieses Verhalten wird zu einem späteren Zeitpunkt dieser Arbeit vertiefend untersucht (siehe Kapitel 3.1.4).

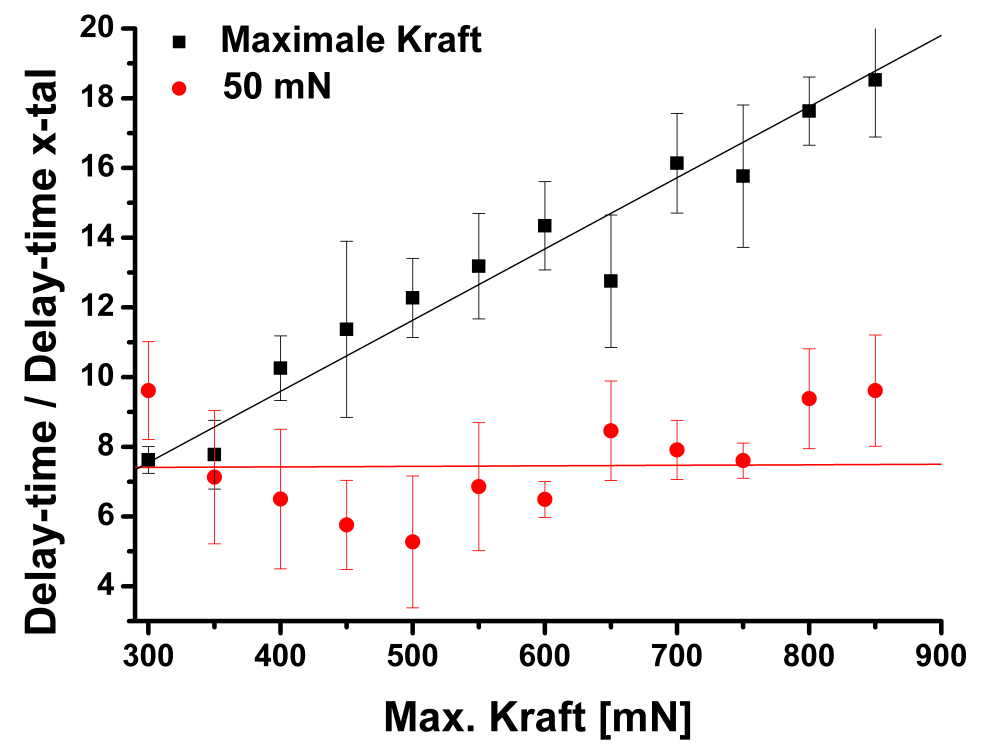

Abbildung 3.20: Vergleich der Delay-time bei $520 \mathrm{~K}$ mit verschiedenen Kräften. Die untere Kraft beim Zurückspringen wurde mit $50 \mathrm{mN}$ konstant gehalten und nur die obere, maximale Kraft wurde systematisch erhöht.

Um die kraftabhängige Dämpfung des amorphen Materials genauer zu untersuchen, wurde bei $520 \mathrm{~K}$ die Delay-time bei unterschiedlichen Verhältnissen zwischen oberer 1

$$
y=y_{0}+\frac{2 A}{\pi} \cdot \frac{w}{4\left(x-x_{c}\right)+w^{2}}
$$

mit $\mathrm{y}=$ Delay-time, $\mathrm{x}=$ Temperatur, $\mathrm{A}, \mathrm{w}=$ Konstanten 
und unterer Kraft bestimmt. Dafür wurde die untere Kraft bei den Versuchen mit $50 \mathrm{mN}$ immer konstant gehalten und die obere Kraft von $300 \mathrm{mN}$ bis $850 \mathrm{mN}$ in $50 \mathrm{mN}$ Abständen erhöht. Die so bestimmte Delay-time ist gegenüber der maximalen Kraft in Abbildung $3.20 \mathrm{zu}$ sehen. Es ist zu erkennen, dass die normierte Delay-time für die untere Kraft (rote Kreise) im Fehlerbereich (bestimmt über Standardabweichung der 6 Wiederholungen) annähernd konstant bei 7,5 bleibt. Die normierte Delay-time für die obere Kraft (schwarze Punkte) hingegen steigt linear mit der Kraft an. Bei $300 \mathrm{mN}$ ist derselbe Wert wie für $50 \mathrm{mN}$ zu sehen $(1,6)$, dieser steigt dann aber bis $850 \mathrm{mN}$ auf 18,5 an. Die beiden Verhalten wurden über Ausgleichsgeraden deutlich gemacht (schwarze und rote Linie).

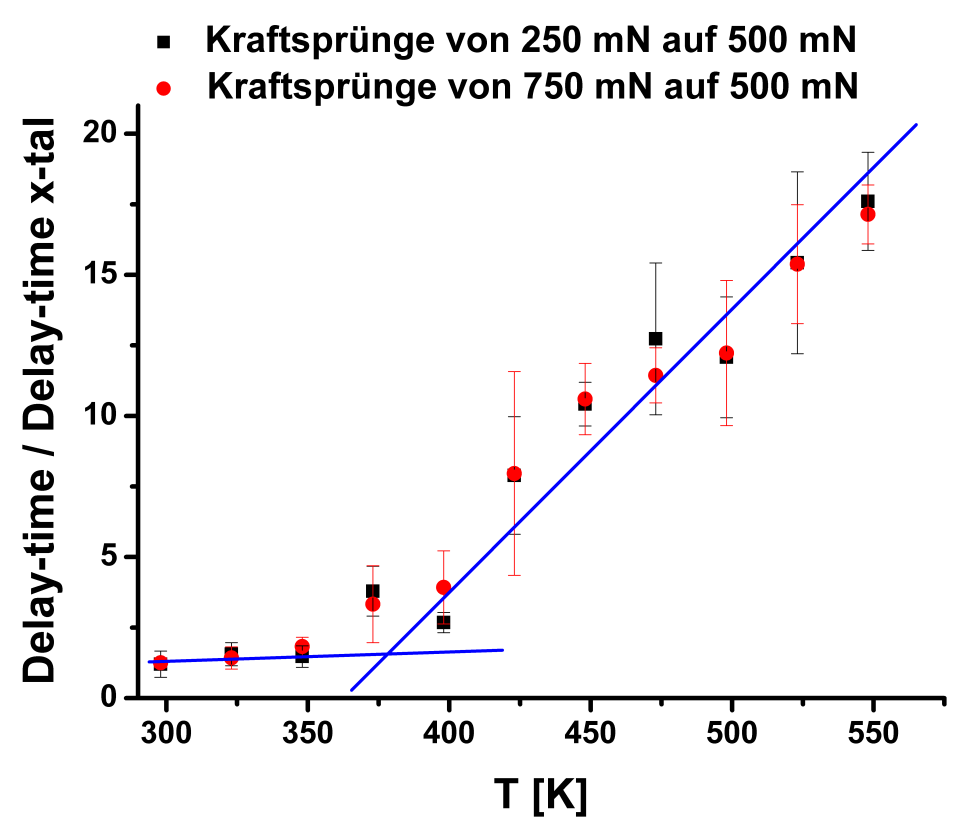

Abbildung 3.21: Vergleich der Delay-time zwischen Creep- und Recoveryteil

Um die Abhängigkeit der Delay-time von der Richtung der Kraftsprünge zu untersuchen, wurde in $25 \mathrm{~K}$ Abständen das Materialverhalten bei Kraftsprüngen von $250 \mathrm{mN}$ bzw. $750 \mathrm{mN}$ auf jeweils $500 \mathrm{mN}$ geprüft. In Abbildung 3.21 sind die Ergebnisse zusammengefasst. In schwarz dargestellt ist die erhaltene Delay-time für die Kraftsprünge mit positiver $(250 \mathrm{mN} \rightarrow 500 \mathrm{mN})$ und in rot mit negativer $(750 \mathrm{mN} \rightarrow 500 \mathrm{mN})$ Kraftänderung. Die Fehler wurden wie in den vorherigen Versuchen bestimmt. Zu erkennen ist, dass kein signifikanter Unterschied in der Delay-time zu beobachten ist, egal ob die Kraftsprünge auf $500 \mathrm{mN}$ hoch oder runter vorgenommen werden. Die Materialantwort 
ist gleich. Außerdem ist zu sehen, dass die Delay-time bis $350 \mathrm{~K}$ im Fehlerbereich konstant ist und erst bei höheren Temperaturen linear ansteigt. Das Verhalten wurde über Ausgleichsgeraden (blaue Geraden) in der Abbildung deutlich gemacht. Die Ausgleichsgeraden schneiden sich bei einer normierten Delay-time von 1,6 und $380 \mathrm{~K}$.

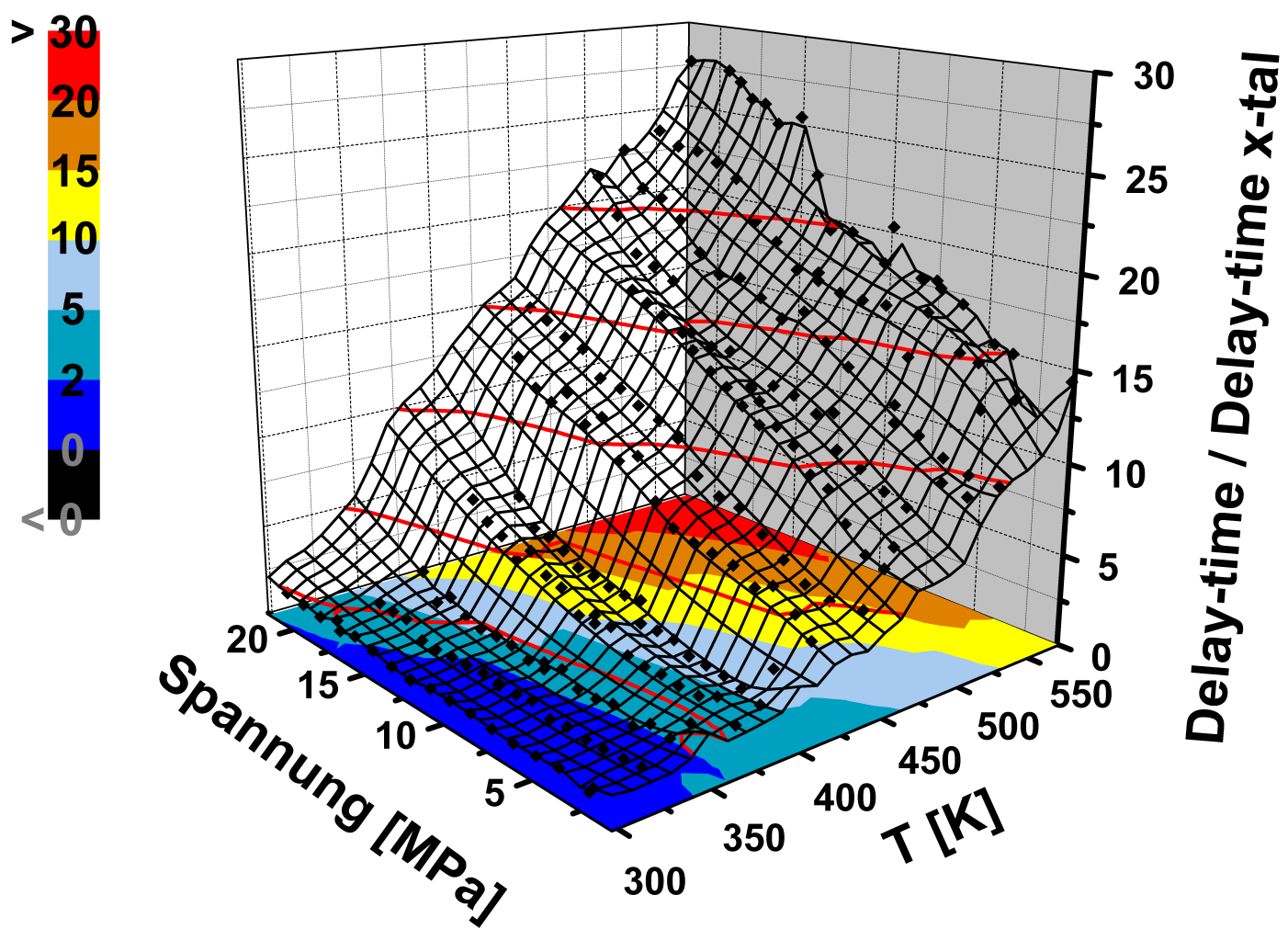

Abbildung 3.22: Spannungs- und Temperaturabhängigkeit der Delay-time (Dämpfung), welche als schwarze Punkte abgebildet und über ein dreidimensionales Netz miteinander verbunden wurde. In der x-y Ebene ist die Veränderung in Abständen von 2 in der Delay-time (rote Linien im Gitternetz) farbig projeziert. Die Farblegende ist links neben der Auftragung zu sehen.

Zur weiteren Untersuchung des Dämpfungsverhaltens wurde sowohl die Spannungsals auch die Temperaturabhängigkeit der Delay-time beobachtet. Hierzu wurde die Delay-time in $25 \mathrm{~K}$ Abständen von Raumtemperatur bis $575 \mathrm{~K}$ in Abhängigkeit der Spannung aufgenommen. Es wurde bei jeder Temperatur die Kraft zwischen 100 mN und $950 \mathrm{mN}$ variiert, wobei jeweils ein Gesamt-Hub von $400 \mathrm{mN}$ verwendet wurde (siehe Kapitel 2.1.4). 
In Abbildung 3.22 sind die so erhaltenen Ergebnisse zu sehen. Es ist die normierte Delay-time (z-Achse) gegenüber der Spannung (y-Achse) und der Temperatur (x-Achse) aufgetragen. Die aus den Messungen bestimmten Delay-Zeiten sind als schwarze Punkte zu sehen, welche über ein dreidimensionales Netz miteinander verbunden und in der $\mathrm{x}-\mathrm{y}$ Ebene farbig projeziert wurden. Die Farblegende ist in der Abbildung links zu sehen. Außerdem wurden die Grenzen zwischen den einzelnen Farbschritten als rote Linien in das Netz eingebunden.

Es ist zu erkennen, dass die Delay-time sowohl mit der Temperatur als auch mit der Spannung ansteigt. Dabei ist bei der kleinsten Spannung der Anstieg erst ab $375 \mathrm{~K}$, bei großen Spannungen über $15 \mathrm{MPa}$ hingegen sofort zu beobachten. In der Nähe der Raumtemperatur ist ein konstantes Verhalten bis zu einer normierten Delay-time von 2 (dunkelblau in der Farbcodierung) zu sehen, so dass in diesem Bereich von einem rein elastischen Verhalten ausgegangen werden kann. Bei höheren Temperaturen und Spannungen sind Bereiche mit ähnlicher Delay-time (eine Farbcodierung) auszumachen, welche annähernd quer im Messbereich und parallel zueinander liegen. Die größten Werte für die Delay-time (über 27) sind bei der größten Temperatur und Spannung zu erkennen. Die zu sehende Streuung in den einzelnen Temperaturbereichen gibt eine gutes Maß für den erhaltenen Fehler wieder, welcher übersichtshalber nicht mit aufgetragen wurde (bestimmt wurde dieser wie in den vorheriegen Versuchen über die Standardabweichung der 6 Kraftwiederholungen). Bei hohen Temperaturen ist die größte Streuung zu erkennen, da die Auswertung durch Temperaturschwankungen erschwert wurde. Der Anstieg der Delay-time mit zunehmender Spannung ist jedoch auch bei diesen Messungen zu erkennen.

Somit ist das Materialverhalten (Dämpfung) der amorphen Probe sowohl spannungs- als auch temperaturabhängig unterhalb der Glasübergangstemperatur zu verfolgen. Dabei ist zu beobachten, dass das Material ein ähnliches Antwortverhalten auf die Temperaturund die Kraftänderung zeigt. Dies wird im Kapitel 4 ausführlich diskutiert. 


\subsubsection{Creep/Recoverymessungen an amorphem PdCuSi zur Untersuchung von temperatur- und spannungsabhängigem Elastizitätsverhalten}

\section{Untersuchung der Glasübergangstemperatur}

Zur genaueren Untersuchung des spannungs- und temperaturabhängigen Dämpfungsverhaltens von metallischen Gläsern wurden Creep/Recoverykurven von amorphem PdCuSi (Kapitel 3.1.4) und ZrAlCu (Kapitel 3.1.5) bei konstanter Heizrate betrachtet. Mit dieser Messmethode können sowohl die Elastizitätsmodule als auch das Verhalten der Glasübergangstemperaturen und der Aktivierungsvolumina der angeregten Prozesse bei verschiedenen äußeren Spannungen und Temperaturen beobachtet und miteinander verglichen werden (siehe Kapitel 2.1.4).

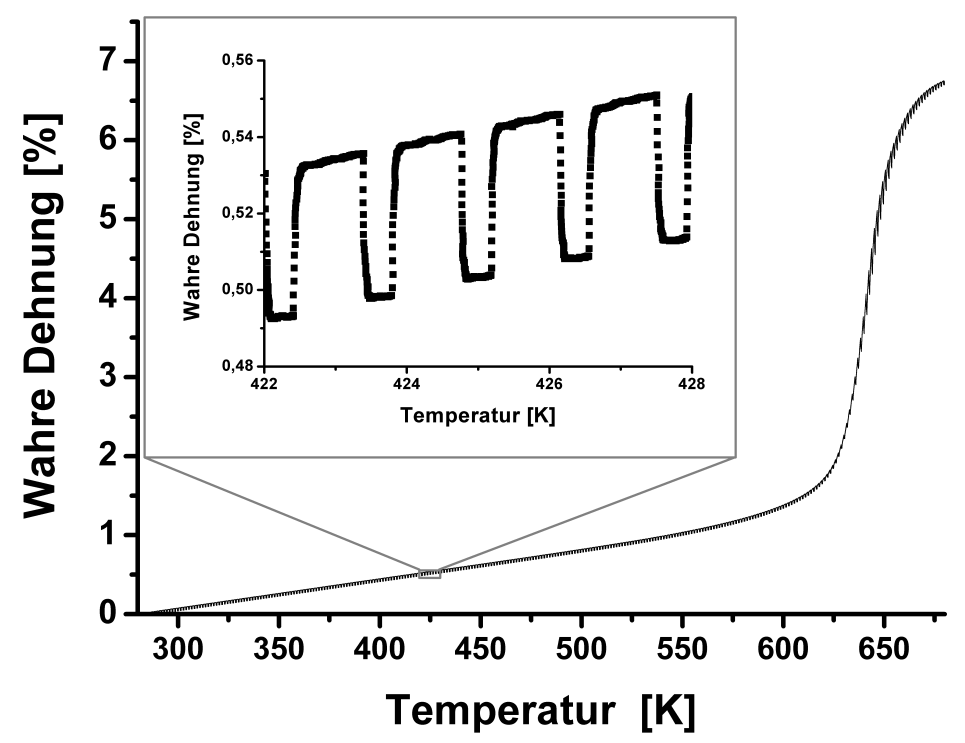

Abbildung 3.23: Messkurve einer Creep/Recoverymessung von PdCuSi mit den Kräften $450 \mathrm{mN}$ und $50 \mathrm{mN}$ bei einer Heizrate von $0,78 \mathrm{~K} / \mathrm{min}$. Der Inset zeigt eine Vergrößerung des Temperaturbereichs von $422 \mathrm{~K}$ bis $428 \mathrm{~K}$.

In Abbildung 3.23 ist die Kurve einer Creep/Recoverymessung von PdCuSi beispielhaft zu sehen. Dabei ist die Probendehnung gegenüber der Temperatur aufgetragen, bei der die Käfte $450 \mathrm{mN}$ (entspricht einer Spannung von $10 \mathrm{MPa}$ ) und $50 \mathrm{mN}$ (1 MPa) sowie eine Heizrate von $0,78 \mathrm{~K} / \mathrm{min}$ verwendet wurden. Im Bereich von Raumtemperatur bis ca. $600 \mathrm{~K}$ ist ein lineares, langsames Ansteigen der relativen Probenlänge zu beobachten. 
Bei höheren Temperaturen ist ein sehr starker Anstieg in der Dehnung zu erkennen, wobei ab $640 \mathrm{~K}$ die Kurve wieder abflacht und eine ähnliche Steigung wie vor $600 \mathrm{~K}$ erreicht wird. Im Inset ist eine Vergrößerung des Temperaturbereichs von $422 \mathrm{~K}$ bis $428 \mathrm{~K}$ dargestellt, das die Probenantwort auf die springende Kraft zeigt. Deutlich ist der lineare Bereich direkt nach dem Kraftsprung zu sehen, mit dem die Elastizitätskonstanten berechnet werden können (siehe Gleichung 1.9). Das Material kriecht anschließend in einen leicht ansteigenden Bereich nach, welcher durch den neuen Kraftsprung beendet wird.

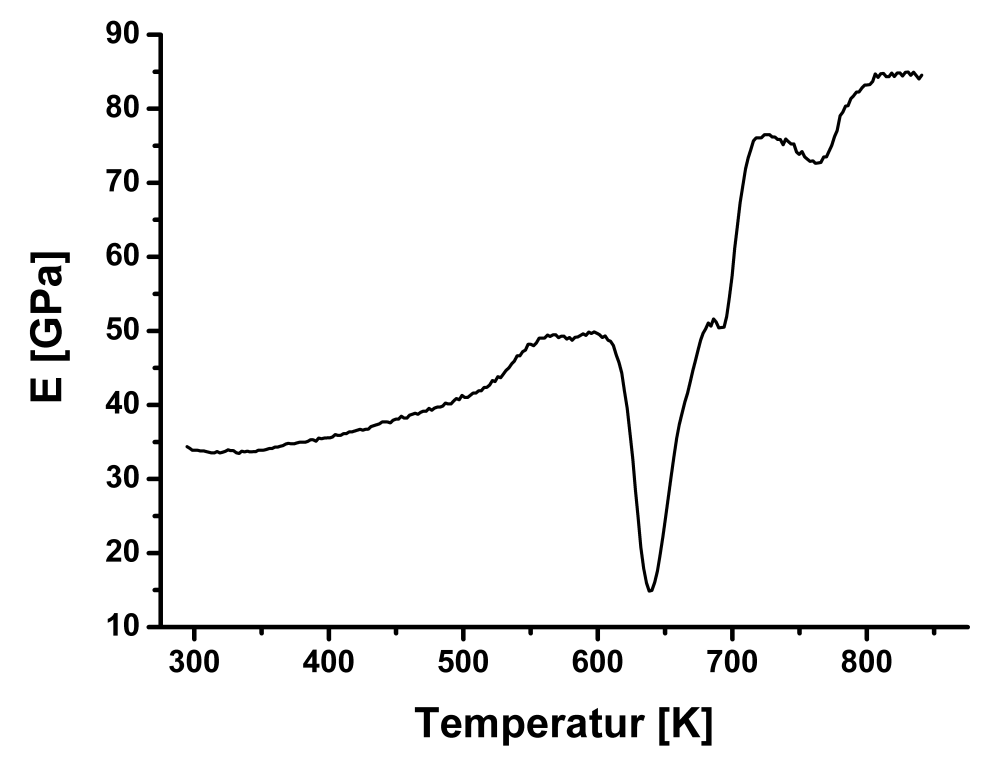

Abbildung 3.24: Aus Creep/Recoverymessungen bestimmter Elastizitätsmodul von $\mathrm{PdCuSi}$ von Raumtemperatur bis $840 \mathrm{~K}$

Aus diesen Messungen wurden die Elastizitätsmodule berechnet. In Abbildung 3.24 ist ein solches Modul beispielhaft über den gesamten Temperaturbereich von Raumtemperatur bis $840 \mathrm{~K}$ zu erkennen. Der Elastizitätsmodul bei Raumtemperatur ist mit $33 \mathrm{GPa}$ bestimmt worden und nimmt mit Erhöhung der Temperatur bis $560 \mathrm{~K}$ auf 49 GPa zu, wo er bis $600 \mathrm{~K}$ annähernd konstant bleibt. Anschließend ist eine starke Abnahme mit einem Minimum bei 640 K (15 GPa) zu erkennen, wonach der Modul wieder stark ansteigt. Bei $685 \mathrm{~K}$ ist ein weiteres Abknicken mit einem neuen Minimum (690 K, 50 GPa) zu sehen, gefolgt von einem weiteren Ansteigen des Moduls bis $720 \mathrm{~K}$ (77 GPa). Ein drittes Abknicken kann mit einem Minimum bei 763 K (73 GPa) beobachtet werden. Bei höheren 
Temperaturen mündet der Modul in ein konstantes Verhalten ab 805 K (85 GPa).

Im Folgenden wird nur der Modul bis zum ersten starken Abknicken und dem folgenden Minimum betrachtet, da die sich anschließende Kristallisation für diese Arbeit nicht von Interesse war.

a)

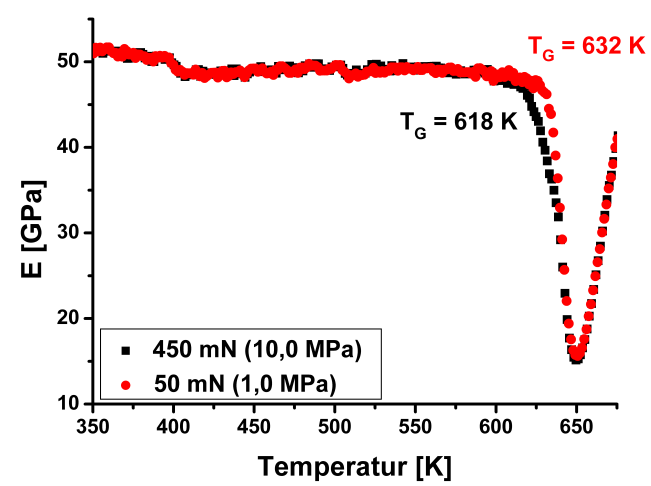

c)

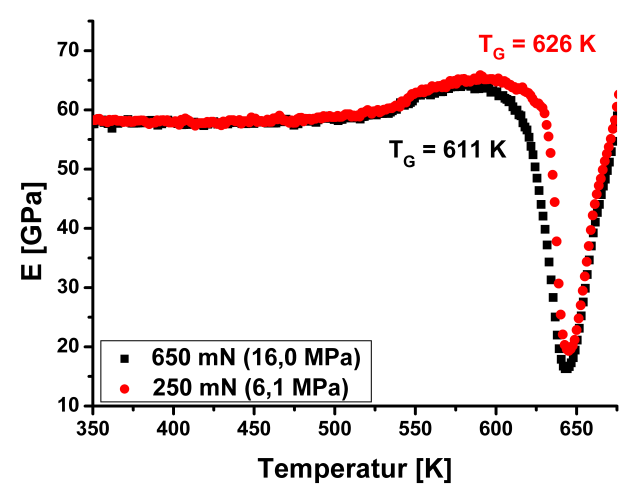

b)

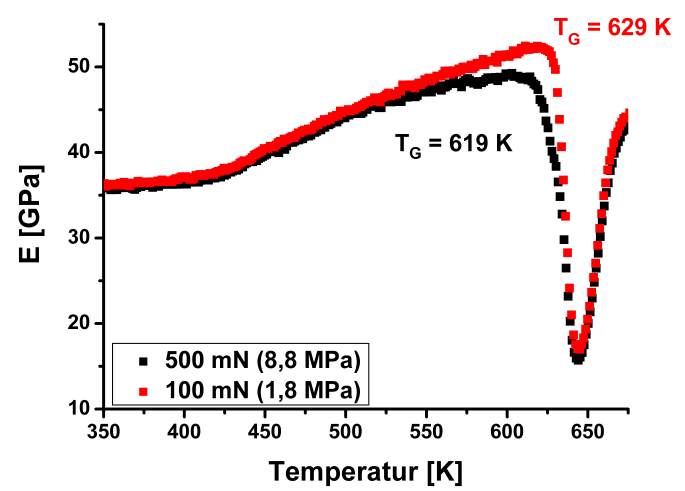

d)

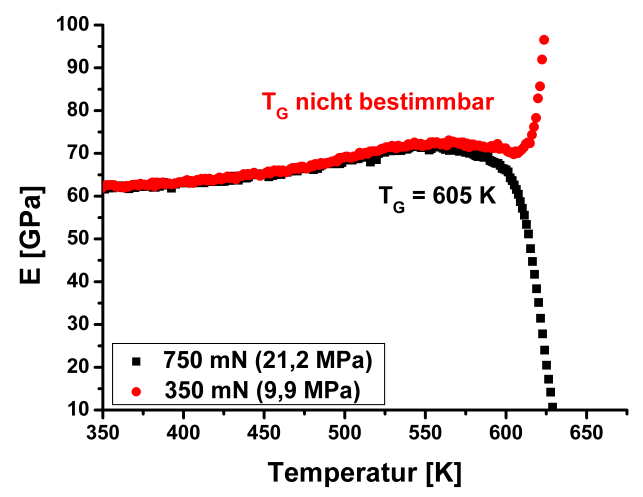

Abbildung 3.25: Vergleich unterschiedlicher Creep/Recoverymessungen von vier Modulberechnungen mit a) $450 \mathrm{mN}(10 \mathrm{MPa}$ - schwarz) und $50 \mathrm{mN}(1 \mathrm{MPa}$ - rot), b) $500 \mathrm{mN}(8,8 \mathrm{MPa}$ - schwarz) und $100 \mathrm{mN}(1,8 \mathrm{MPa}$ - rot), c) $650 \mathrm{mN}(16,0 \mathrm{MPa}$ - schwarz) und $250 \mathrm{mN}(6,1 \mathrm{MPa}$ - rot) und d) $750 \mathrm{mN}(21,2 \mathrm{MPa}$ - schwarz) und $350 \mathrm{mN}(9,9 \mathrm{MPa}$ - rot)

In Abbildung 3.25 ist ein Vergleich von vier Modulberechnungen aus unterschiedlichen Creep/Recoverymessungen bei den Kräften a) $450 \mathrm{mN}$ - $10 \mathrm{MPa}$ (schwarz) und $50 \mathrm{mN}$ $1 \mathrm{MPa}$ (rot), b) $500 \mathrm{mN}$ - 8,8 MPa (schwarz) und $100 \mathrm{mN}$ - 1,8 MPa (rot), c) $650 \mathrm{mN}$ 16,0 MPa (schwarz) und $250 \mathrm{mN}$ - 6,1 MPa (rot) und d) $750 \mathrm{mN}-21,2 \mathrm{MPa}$ (schwarz) und $350 \mathrm{mN}$ - 9,9 $\mathrm{MPa}$ (rot) zu sehen. Im Bildteil a) ist ein konstanter Verlauf der Module von $51 \mathrm{GPa}$ sowohl für die obere als auch die untere Kraft bis zu $400 \mathrm{~K}$ zu erkennen. Danach sinkt der Modul auf 48 GPa ab und verläuft weiter konstant bis $600 \mathrm{~K}$. 
$\mathrm{Zu}$ höheren Temperaturen sinken für beide Kräfte die Module ab, wobei ein früheres Abknicken bei der größeren Kraft zu beobachten ist. Über eine Onset-Methode lässt sich bei $450 \mathrm{mN}$ eine Glasübergangstemperatur von $618 \mathrm{~K}$ und bei $50 \mathrm{mN}$ von $632 \mathrm{~K}$ bestimmen. Oberhalb von $\mathrm{T}_{G}$ fallen die Module bis $650 \mathrm{~K}(16 \mathrm{MPa}) \mathrm{ab}$, wobei sie ab $640 \mathrm{~K}$ wieder überlappend verlaufen. Oberhalb von $650 \mathrm{~K}$ ist ein erneutes Ansteigen des Moduls zu beobachten.

Im Bildteil b) verlaufen die Module der beiden Kräfte bei Raumtemperatur ebenfalls zusammen $(\mathrm{E}=36 \mathrm{GPa})$, es ist jedoch ab $420 \mathrm{~K}$ ein Ansteigen zu erkennen und ab $525 \mathrm{~K}$ ist ein kleinerer Modul bei größerer Kraft zu beobachten. Ein starkes Abknicken und eine Reduzierung der Module kann bei $\mathrm{T}_{G}$ ebenfalls beobachtet werden (619 K bei $500 \mathrm{mN}$ und $629 \mathrm{~K}$ bei $100 \mathrm{mN}$ ). Das Minimum in der Kurve ist bei $644 \mathrm{~K}$ zu sehen, wobei in den Modulen ab $635 \mathrm{~K}$ kein Unterschied mehr zu bestimmen ist. Oberhalb von $644 \mathrm{~K}$ steigt der Modul wieder an.

Bei größerer Spannung (Bildteil c) ist ebenfalls ein konstanter Verlauf beider Module von Raumtemperatur bis $530 \mathrm{~K}$ und anschließend ein Ansteigen zu beobachten. Wie bei den voherigen Bildteilen liegt der Modul bei der größeren verwendeten Kraft unter der der niedrigeren. Die Glasübergangstemperatur kann für $650 \mathrm{mN}$ bei $611 \mathrm{~K}$ und für $250 \mathrm{mN}$ bei $626 \mathrm{~K}$ bestimmt werden. Bei höheren Temperaturen sinken die Module wie bei den voherigen Messungen ab, wobei das Minimum bei $645 \mathrm{~K}$ zu sehen ist. Die Module verlaufen auch erst nach Erreichen des Minimums wieder überlappend, wenn ein erneutes Ansteigen zu beobachten ist.

Im Bildteil d) ist ein ähnlicher Verlauf unterhalb von $\mathrm{T}_{G}$ zu erkennen. Die Module liegen bei Raumtemperatur höher als bei den vorigen Messungen. Sie steigen auch mit Beginn der Messung leicht an, wobei ein Maximum für die größere Kraft bei $557 \mathrm{~K}$ zu sehen ist. Bei der kleineren Kraft steigt das Modul bis $568 \mathrm{~K}$ weiter an. Für die schwarze Kurve kann die Glasübergangstemperatur bei $605 \mathrm{~K}$ bestimmt werden, wobei ebenfalls bei höheren Temperaturen ein starkes Abknicken beobachtet werden kann. Ein Minimum ist nicht zu erkennen, da die Probe bei $630 \mathrm{~K}$ gerissen ist. Für die geringere Kraft ist der Modul nur bis $605 \mathrm{~K}$ bestimmbar. Bei höheren Temperaturen nimmt der Modul scheinbar sehr stark zu, was unphysikalisch ist, da die Probe, erkennbar an der Dehnungsänderung, weicher wird. Die Relaxationszeiten für die größere Kraft waren in diesem Fall zu lang, so dass die Haltezeiten (siehe Kapitel 2.1.4) bei hohen Temperaturen 
zu kurz waren. Dementsprechend wurde der Modul der niedrigeren Kraft nicht weiter verwendet.

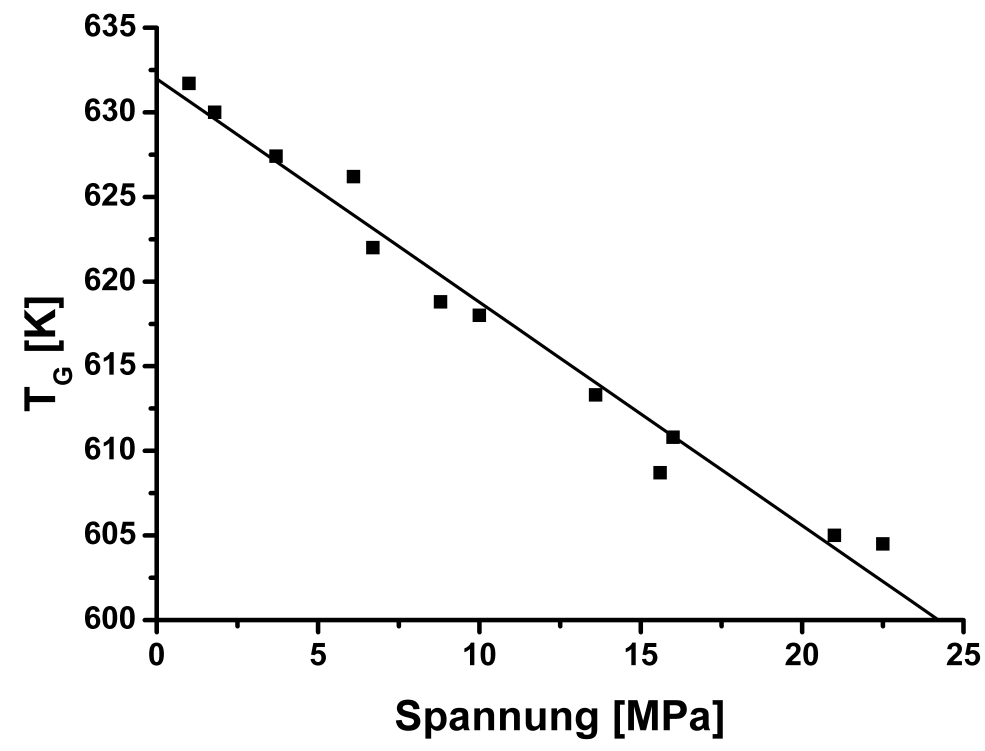

Abbildung 3.26: Spannungsabhängige Glasübergangstemperatur von PdCuSi

Durch diese Messungen konnte bei verschiedenen Kräften die Glasübergangstemperatur bestimmt und verfolgt werden. In Abbildung 3.26 ist diese gegenüber der angelegten, äußeren Spannung aufgetragen. Es ist zu sehen, dass sich $\mathrm{T}_{G}$ spannungsabhängig verhält und mit größerer Spannung zu kleineren Temperaturen verschoben wird, wobei ein linearer Zusammenhang in dem untersuchten Spannungsbereich zu beobachten ist. Insgesamt ist eine Differenz von $27 \mathrm{~K}$ bei einer Veränderung der Spannung von 21,5 MPa zu erkennen. Dies entspricht einer Abnahme von $\mathrm{T}_{G}$ von 1,3 $\mathrm{K}$ pro MPa.

\section{Untersuchung des Aktivierungsvolumens}

Die zugrundeliegenden Prozesse, welche zum Erweichen des amorphen Festkörpers beitragen, sind in der Literatur nicht abschließend diskutiert. ${ }^{[14,27,62,64-67]}$ Molekulardynamische Simulationen lassen Rückschlüsse auf clusterähnliche Bereiche zu, welche mitverantwortlich für plastische Verformungen sind (siehe Kapitel 1.4.4). Um experimentell etwas über die Größe dieser Bereiche zu erfahren, wird in der Literatur die Angabe des Aktivierungsvolumens vorgeschlagen, ${ }^{[27,66,67]}$ wobei bisher nur wenige experimentelle Daten von den Abmaßen der verantwortlichen Heterogenitäten vorliegen. Um das Aktivierungsvolumen bestimmen zu können, werden in dieser Arbeit die zuvor beschriebenen 
Creep/Recoverymessungen verwendet und wie in den Kapiteln 1.3.1.3 und 2.1.4 beschrieben ausgewertet. Hierfür ist es notwendig, dass das Material dehnungsratensensitiv ist, da die Gleichung 1.14 nur bei einer plastischen Deformation anzuwenden ist. In Abbildung 3.27 ist die nach Gleichung 1.15 berechnete Dehnungsratensensitivität zur Messung aus Abbildung 3.25 a) beispielhaft dargestellt.

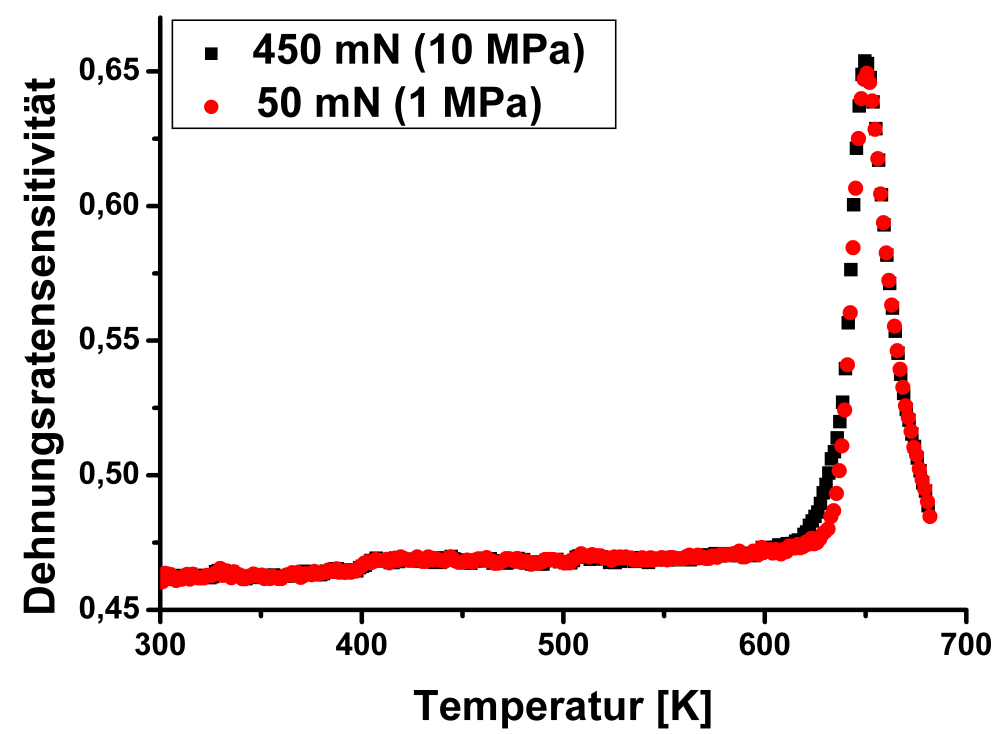

Abbildung 3.27: Berechnete Dehnungsratensensitivität zur in Abbildung 3.25 a) dargestellten Messung mit den verwendeten Kräften 450 mN (schwarz) und $50 \mathrm{mN}($ rot $)$

Dabei wurde der Temperaturbereich von $300 \mathrm{~K}$ bis $700 \mathrm{~K}$ bei den Kräften $450 \mathrm{mN}$ (schwarz) und $50 \mathrm{mN}$ (rot) abgebildet. Unterhalb von $600 \mathrm{~K}$ ist ein konstantes und somit kein dehnungsratensensitives Verhalten zu beobachten, da keine plastische Verformung des Materials erkennbar ist. Erst im Temperaturbereich in der Nähe von $\mathrm{T}_{G}$ ist ein starker Anstieg mit einem Maximum bei $650 \mathrm{~K}$ zu sehen. Hiernach fällt die Kurve wieder ab. Im Temperaturbereich von $600 \mathrm{~K}$ bis $640 \mathrm{~K}$ ist ein Versatz der Kurven zu erkennen, wobei die Kurve der größeren Kraft einen stärkeren Anstieg zeigt und somit über der der niedrigeren liegt. Somit kann im Temperaturbereich, in dem kein konstantes Verhalten zu erkennen ist, (600 K bis $700 \mathrm{~K}$ ) ein plastisches Verhalten des Materials beobachtet werden. Im Folgenden werden deshalb nur die in diesem Bereich beobachteten Veränderungen im Aktivierungsvolumen beschrieben. 


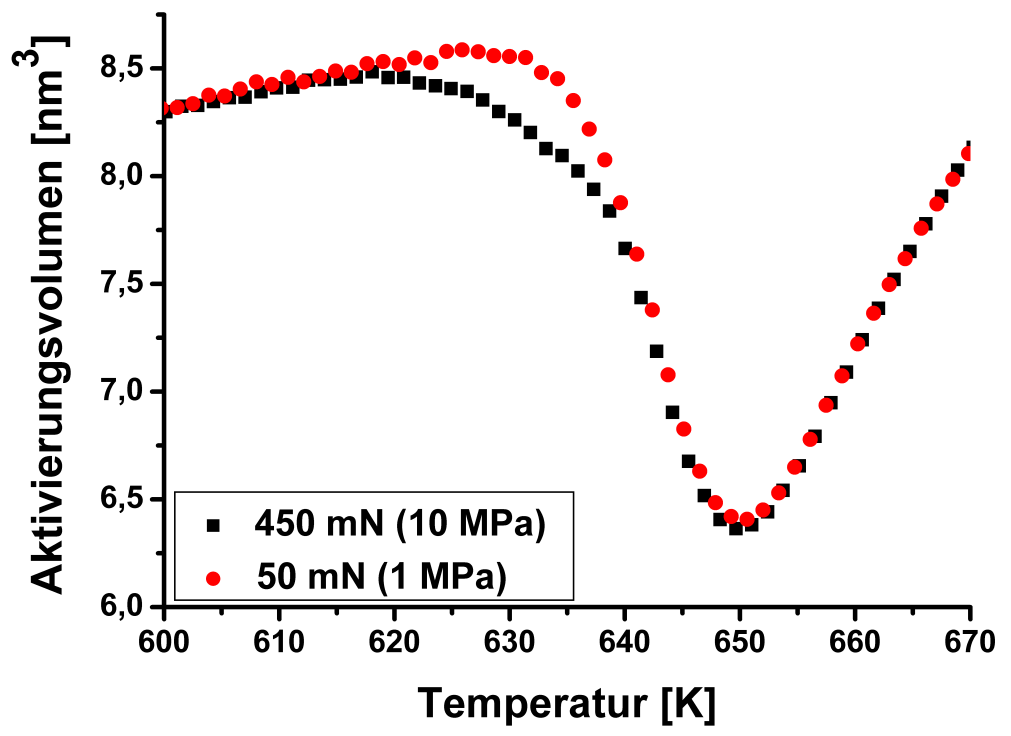

Abbildung 3.28: Berechnetes Aktivierungsvolumen zur in Abbildung 3.25 a) dargestellten Messung mit den verwendeten Kräften $450 \mathrm{mN}$ (schwarz) und $50 \mathrm{mN}$ (rot).

In Abbildung 3.28 ist das nach Gleichung 1.14 berechnete Aktivierungsvolumen zur Messung aus Abbildung 3.25 a) beispielhaft dargestellt.

$\mathrm{Zu}$ erkennen ist ein leicht ansteigender Verlauf des Aktivierungsvolumens unterhalb von $\mathrm{T}_{G}$, wobei die bestimmten Volumina für beide Kräfte zusammen verlaufen. Bei Temperaturen nahe $\mathrm{T}_{G}$ ist ein Auseinanderlaufen der Kurven zu beobachten. Zuerst sinkt das Aktivierungsvolumen für die größere Kraft mit einem Maximum bei $618 \mathrm{~K}$ $\left(8,48 \mathrm{~nm}^{3}\right.$ - entspricht einer Zahl von ca. 560 Atomen) ab, wobei es für die kleinere Kraft bis $625 \mathrm{~K}\left(8,58 \mathrm{~nm}^{3}\right.$ - entspricht einer Zahl von ca. 570 Atomen) weiter ansteigt und dann ebenfalls wieder kleiner wird. Somit kann eine klare Verschiebung des Maximums in der Temperatur festgestellt werden und ebenfalls im Aktivierungsvolumen, wobei dieser Unterscheid deutlich kleiner ist. Im Bereich der unterkühlten Schmelze nimmt die Differenz im Aktivierungsvolumen zuerst zu, so dass bei $634 \mathrm{~K}$ ein Unterschied von $0,32 \mathrm{~nm}^{3}$ ( $\approx 20$ Atome) zwischen den Kräften bestimmt werden kann. Danach nimmt die Differenz ab und die Kurven überlappen ab dem Beginn der Kristallisation bei $650 \mathrm{~K}$ wieder. Ein gemeinsames Minimum ist bei $650 \mathrm{~K}$ (6,37 $\mathrm{nm}^{3}$ - 420 Atome) zu sehen, wonach das errechnete Aktivierungsvolumen wieder ansteigt. 


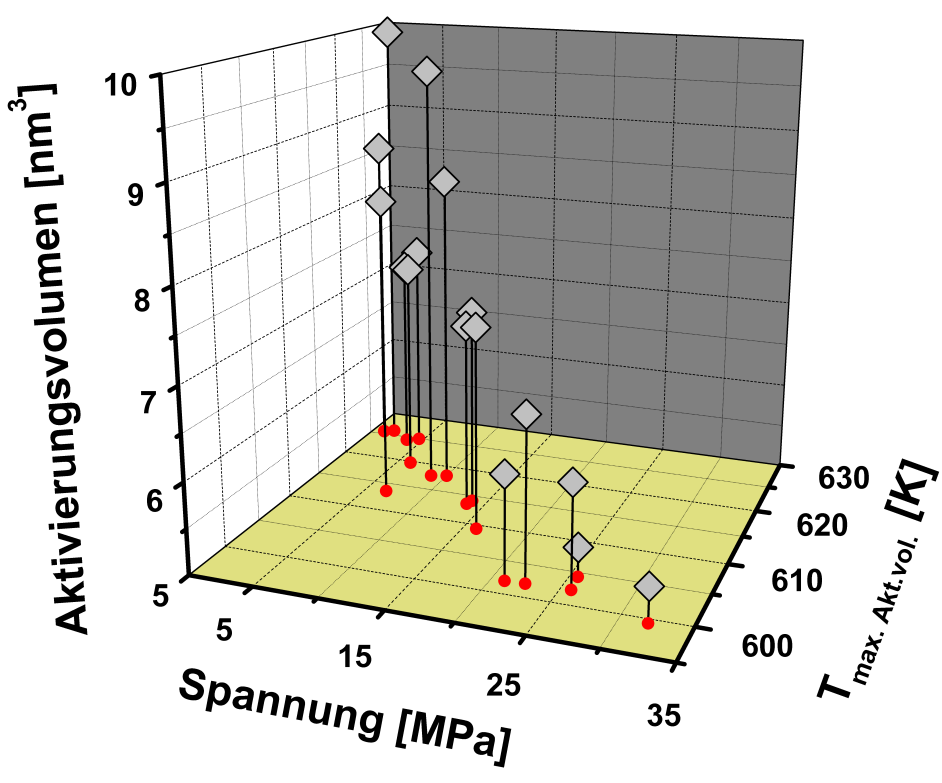

Abbildung 3.29: Maximal bestimmtes Aktivierungsvolumen und die zugehörige Temperatur in Abhängigkeit der äußeren Spannung

Bei der Betrachtung von weiteren Kräften kann ein spannungsabhängiges Aktivierungsvolumen beobachtet werden. In Abbildung 3.29 sind sowohl die maximal erhaltenen Aktivierungsvolumina als auch die zugehörigen Temperaturen gegenüber der angelegten äußeren Spannung aufgetragen. Die bestimmten Werte sind als rote Punkte in der $\mathrm{x}-\mathrm{y}$-Ebene übersichtshalber abgebildet.

Es ist sowohl eine Abnahme des Aktivierungsvolumens als auch der maximalen Temperatur (Umkehrpunkt in der Kurve) mit Erhöhung der äußeren Spannung zu erkennen. Dabei ist eine Reduzierung des Volumens um 4,63 nm (310 Atome) von 9,95 nm (660 Atome) auf 5,32 $\mathrm{nm}^{3}$ (350 Atome) zu beobachten, wobei die Spannung von $1 \mathrm{MPa}$ auf $32 \mathrm{MPa}$ erhöht wurde. Ebenfalls ist ein großer Temperaturunterschied von $27 \mathrm{~K}$ (626 K zu $599 \mathrm{~K})$ beim maximalen Aktivierungsvolumen zu sehen.

Um diese Auswertmethode zu verifizieren und das Aktivierungsvolumen in der unterkühlten Schmelze genauer zu untersuchen, wurde die Dehnungsrate im Bereich der plastischen Deformation (in der Nähe von $\mathrm{T}_{G}$ ) bei drei verschiedenen externen Spannungen (9,5 MPa, 13,9 MPa und 15,9 MPa) bei jeweils gleicher Dehnung betrachtet. 
Dazu wurde, wie im Kapitel 1.3.1.2 (Gleichung 1.12) beschrieben, die wahre Dehnung berechnet und gegenüber der berechneten Dehnungsrate (Kapitel 2.1.4, Gleichung 2.1) aufgetragen (siehe Abb. 3.30).

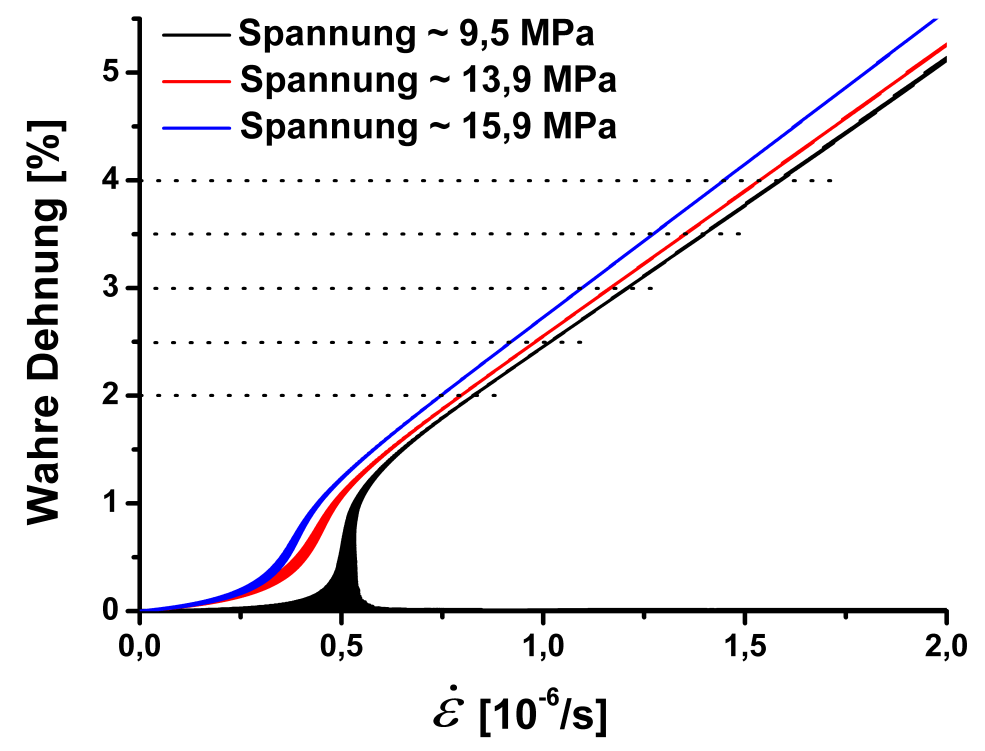

Abbildung 3.30: Wahre Dehnung gegenüber der berechneten Dehnungsrate zur Bestimmung des Aktivierungsvolumens in der unterkühlten Schmelze

Es wurden die Dehnungsraten für die Spannungen 9,5 MPa (schwarz), 13,9 MPa (rot) und 15,9 MPa (blau) bei den Dehnungen 2,0 \%, 2,5 \%, 3,0 \%, 3,5 \% und 4,0 \% bestimmt. Diese sind in Abbildung 3.30 als Schnittpunkte der gestrichelten Linien mit den jeweiligen Kurven zu sehen. Kleinere Dehnungen wurden nicht betrachtet, um sicherzustellen, dass nur der nichtlineare, plastische Bereich berücksichtigt wird. Bei größeren Dehnungen bestand die Gefahr, dass das Material anfing zu kristallisieren. Um aus den erhaltenen Werten ein Aktivierungsvolumen zu bestimmen, wurde der nichtplastische Bereich (unterhalb von $\mathrm{T}_{G}$ ) subtrahiert, indem die Dehnungsrate erst ab Beginn der plastischen Verformung bestimmt wurde. Die so erhaltenen Dehnungsraten wurden halblogarithmisch gegenüber den wahren Spannungen aufgetragen (siehe Abb. 3.31). Unter der Annahme, dass ein lineares Verhalten vorliegt, kann somit für jede betrachtete Dehnung eine Steigung bestimmt werden, welche der Berechnung des Aktivierungsvolumens dient. Hierfür wurde Gleichung 1.14 unter der Annahme verwendet, dass die Temperatur konstant $\mathrm{T}_{G}(620 \mathrm{~K})$ betrug. 


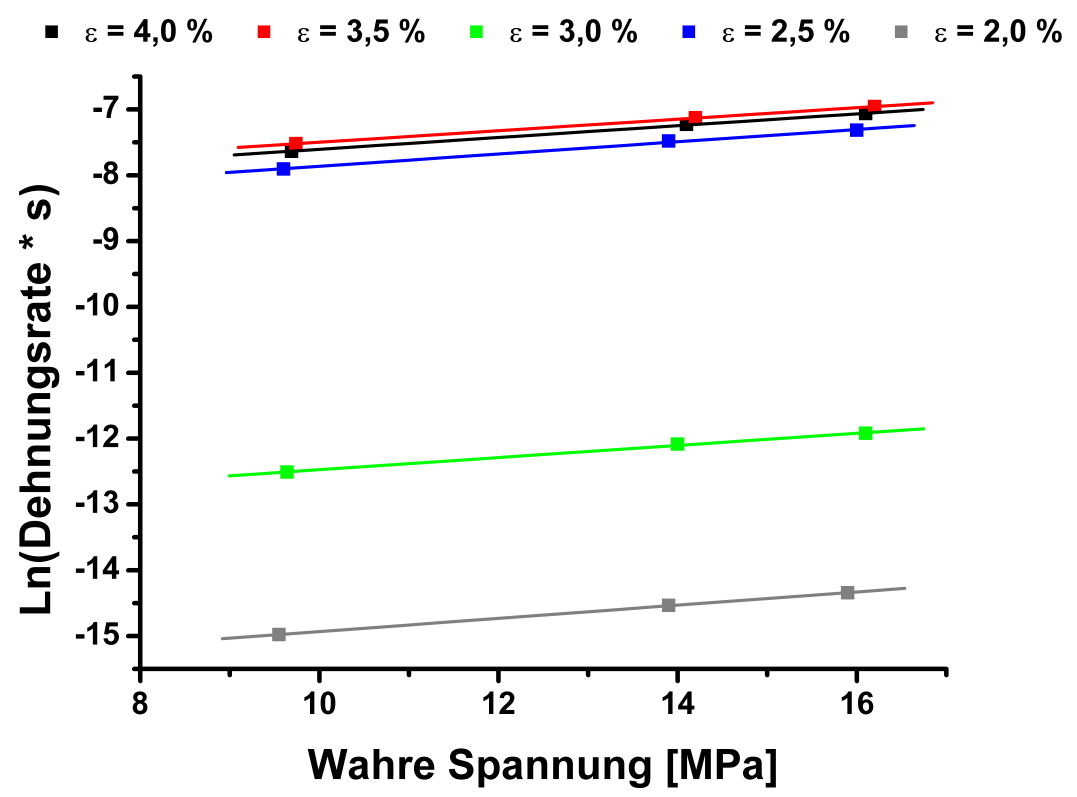

Abbildung 3.31: Halblogarithmische Auftragung der Dehnungsrate bei konstanter Dehnung gegenüber der wahren Spannung zur Bestimmung des Aktivierungsvolumens in der unterkühlten Schmelze.

In Abbildung 3.31 ist diese Auftragung zur Bestimmung der Steigungen $m$ und in Tabelle 3.1 die Ergebnisse zu sehen. Es ist zu erkennen, dass die Steigungen mit zunehmender Dehnung abnehmen.

\begin{tabular}{|c|c|}
\hline$\epsilon[\%]$ & $\mathrm{m}\left[\mathrm{MPa}^{-1}\right]$ \\
\hline 2,0 & 1,00 \\
\hline 2,5 & 0,93 \\
\hline 3,0 & 0,90 \\
\hline 3,5 & 0,89 \\
\hline 4,0 & 0,87 \\
\hline
\end{tabular}

Tabelle 3.1: Bestimmte Steigungen zur Berechnung des Aktivierungsvolumens aus der Auftragung in Abbildung 3.31

Die so erhaltenen Werte wurden mit Hilfe der Gleichung 1.14 direkt zur Bestimmung des Aktivierungsvolumens verwendet, welches in Abbildung 3.32 in Abhängigkeit der Dehnung aufgetragen wurde. 


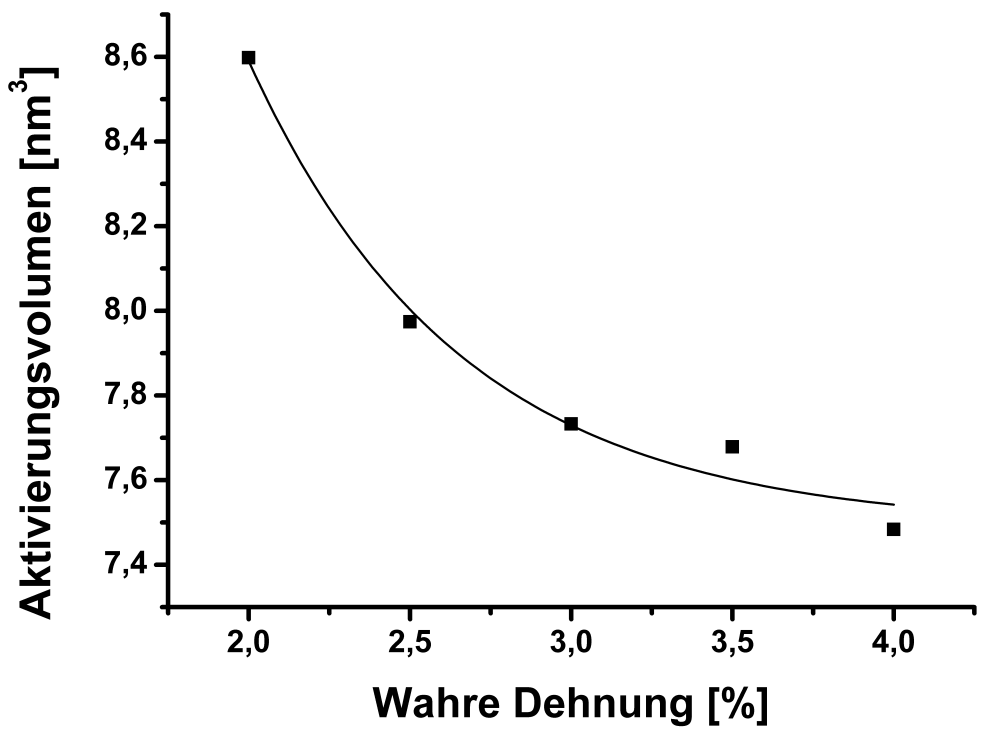

Abbildung 3.32: Aktivierungsvolumen in Abhängigkeit der wahren Dehnung in der unterkühlten Schmelze

Mit zunehmender Dehnung ist eine Abnahme des Aktivierungsvolumens zu erkennen. Bei einer Dehnung von 2,0 \% wurde ein Volumen von 8,59 $\mathrm{nm}^{3}$ (570 Atome) bestimmt, welches zu 4,0\% auf 7,48 $\mathrm{nm}^{3}$ (490 Atome) abnimmt. In der Abbildung wurde das Verhalten über einen exponentiellen Abfall (schwarzen Kurve) verdeutlicht, für welchen die Gleichung

$$
\Omega=\Omega_{\infty}+A \cdot e^{\left(-\frac{\epsilon}{\tau}\right)}
$$

verwendet wurde (mit $\Omega_{\infty}=7,49 \mathrm{~nm}^{3}, \mathrm{~A}=23,2 \mathrm{~nm}^{3}$ und $\tau=0,0066$ ). Dies kann als Indiz für ein Hochtemperatur-Barrierenlimit für das Aktivierungsvolumen von 7,49 $\mathrm{nm}^{3}$ (490 Atome) gewertet werden. Diese These wird im Kapitel 4 genauer diskutiert. 


\subsubsection{Creep/Recoverymessungen an amorphem ZrAlCu zur Untersuchung von temperatur- und spannungsabhängigem Elastizitätsverhalten}

\section{Untersuchung der Glasübergangstemperatur}

Im Vergleich zu den Messungen am fragilen Glasbildner PdCuSi wurde der starke Glasbildner $\mathrm{Zr}_{65,0} \mathrm{Al}_{7,5} \mathrm{Cu}_{27,5}$ untersucht. Hierzu wurden wie im Kapitel 2.1.4 beschrieben Creep/Recoverymessungen mit einer konstanten Heizrate durchgeführt. Dabei wurde darauf geachtet, dass die Messbedingungen identisch zu denen mit PdCuSi waren. Es wurde aber zur Vermeidung von Oxidation in einer geschlossenen Stickstoffatmosphäre anstatt nur mit einem kontinuierlichen Stickstoffstrom gearbeitet (siehe Kapitel 2.1.1).

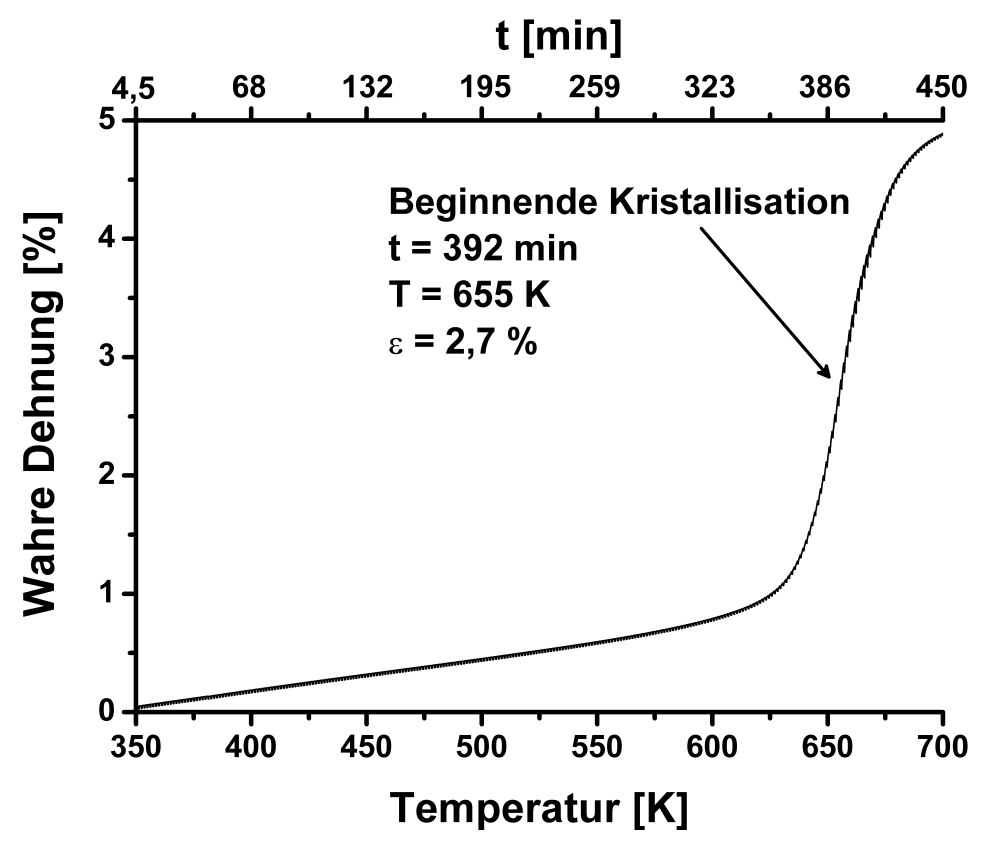

Abbildung 3.33: Relative Probenlänge gegenüber der Probentemperatur bzw. der Versuchszeit bei den verwendeten Kräften von $500 \mathrm{mN}$ und $100 \mathrm{mN}$

Beispielhaft ist eine Messkurve in Abbildung 3.33 zu sehen, bei der die relative Probenlänge (Dehnung) gegenüber der Probentemperatur bzw. der Versuchszeit aufgetragen wurde. Für den Creepteil wurde eine Kraft von 500 mN (4,9 MPa) und für den Recoveryteil von $100 \mathrm{mN}$ (1,0 MPa) verwendet. Zu erkennen ist, dass die Dehnung im Temperaturbereich unterhalb von $\mathrm{T}_{G}$ linear mit der Temperatur ansteigt, aber eine sehr kleine Steigung aufweist. Im Bereich der Glasübergangstemperatur (640 K) ist ein starker Anstieg der Probenlänge zu beobachten, wobei ab 655 K (392 min, 2,7 \% Dehnung) die Kurve wieder 
abflacht. Hier kann der Beginn der Kristallisation bestimmt werden. Dieser Punkt ist in der Abbildung durch einen Pfeil markiert. Aus diesen Messungen konnten wie im Kapitel 2.1.4 beschrieben, die Elastizitätsmodule der einzelnen Messungen bestimmt werden. Beispielhaft sind in Abbildung 3.34 zwei Messungen dargestellt.

a)

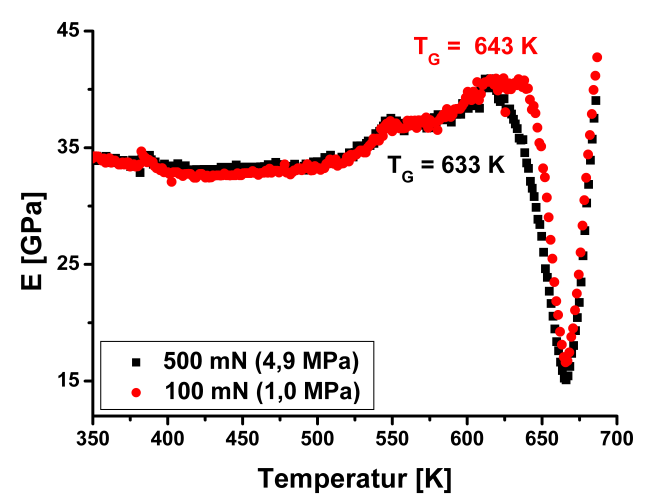

b)

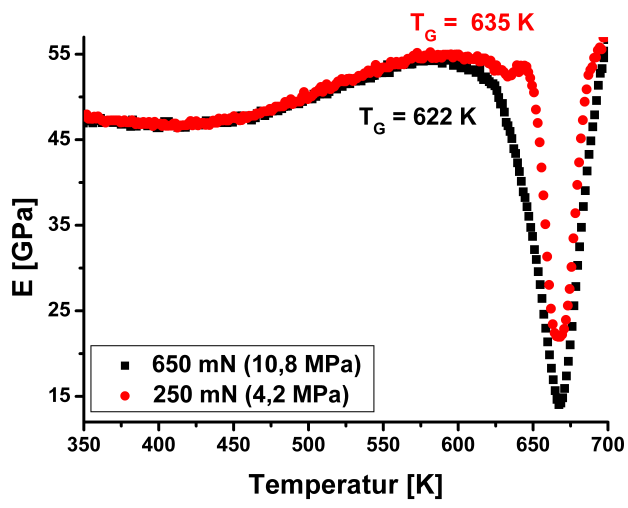

Abbildung 3.34: Vergleich von zwei Modulberechnungen von $\mathrm{ZrAlCu}$ a) $500 \mathrm{mN}$ (4,9 MPa - schwarz) und $100 \mathrm{mN}(1,0 \mathrm{MPa}$ - rot), b) $650 \mathrm{mN}$ (10,8 MPa - schwarz) und $250 \mathrm{mN}(4,2 \mathrm{MPa}$ - rot)

Im Bildteil a) ist der Modul für die Messung mit den Kräften $500 \mathrm{mN}$ (4,9 MPa schwarz) und $100 \mathrm{mN}(1,0 \mathrm{MPa}$ - rot) zu sehen. Die Module haben bei Raumtemperatur einen Wert von $33 \mathrm{MPa}$ und verlaufen sowohl überlappend als auch konstant mit der Temperatur bis $500 \mathrm{~K}$ und steigen dann bis $610 \mathrm{~K}$ an. Ab $617 \mathrm{~K}$ fällt der Modul für die größere Kraft ab, wobei für die kleinere Kraft ein konstantes Verhalten bis $640 \mathrm{~K}$ bestimmt werden konnte, bis auch dieser Modul kleiner wird. Ein gemeinsames Minimum ist bei $665 \mathrm{~K}$ zu sehen, wobei für die größere Kraft ein kleinerer Modul bestimmt wurde. Bei höheren Temperaturen steigen die Module wieder an und überlappen erneut. Über eine Onset-Methode konnte die Glasübergangstemperatur bei $643 \mathrm{~K}(100 \mathrm{mN})$ und $633 \mathrm{~K}(500 \mathrm{mN})$ bestimmt werden. Im Bildteil b) ist ein ähnlicher Verlauf zu beobachten. Es sind die Elastizitätsmodule für die Kräfte $650 \mathrm{mN}(10,8 \mathrm{MPa}$ - schwarz) und $250 \mathrm{mN}$ (4,2 MPa - rot) zu sehen. Unterhalb von $\mathrm{T}_{G}$ überlappen die Module und zeigen bis $450 \mathrm{~K}$ einen konstanten Verlauf, steigen dann bis $580 \mathrm{~K}$ an, woraufhin der Modul für die größere Kraft wieder kleiner wird. Für die kleinere Kraft sinkt der Modul erst ab $620 \mathrm{~K}$ wieder ab. Ein Minimum wird von beiden Modulen bei $667 \mathrm{~K}$ durchlaufen, wobei ebenfalls ein größerer Wert für den 
Elastizitätsmodul bei der kleineren Kraft bestimmt wurde. Bei höheren Temperaturen steigen die Module wieder an, wobei jedoch erst bei Temperaturen oberhalb von $700 \mathrm{~K}$ eine erneute Überlappung der Module auftritt. Die Glasübergangstemperatur wurde bei $635 \mathrm{~K}(250 \mathrm{mN})$ und $622 \mathrm{~K}(650 \mathrm{mN})$ bestimmt.

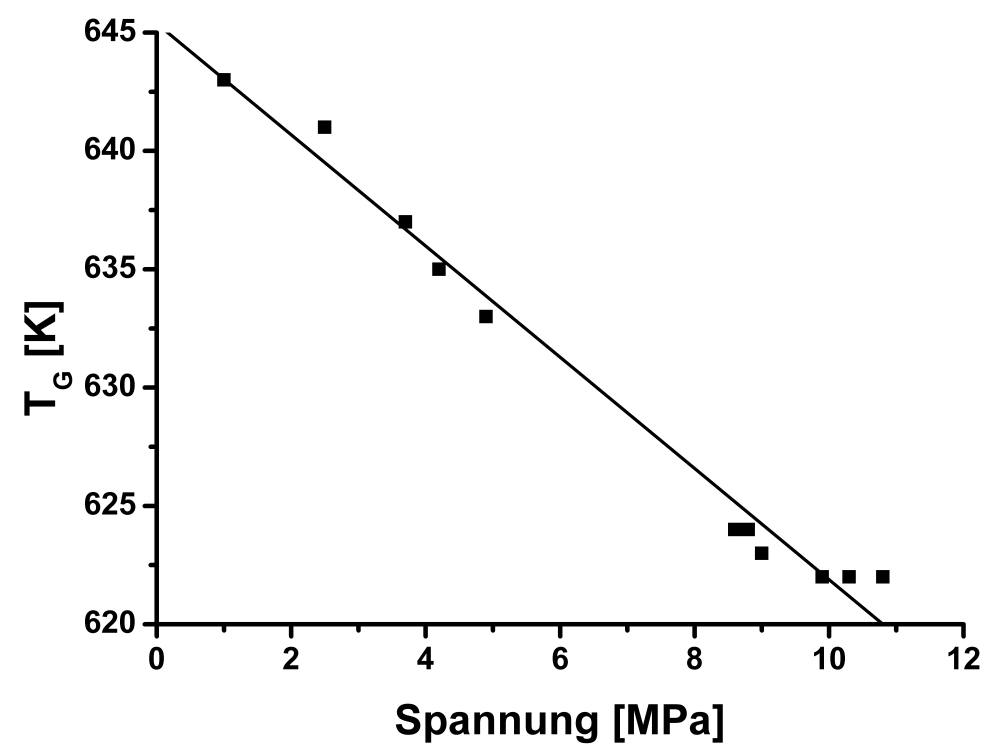

Abbildung 3.35: Spannungsabhängige Glasübergangstemperatur von $\mathrm{ZrAlCu}$

Mit Hilfe dieser Messungen wurden die Glasübergangstemperaturen bestimmt und in Abhängigkeit der äußeren Spannung aufgetragen (siehe Abb. 3.35). Es ist eine Abnahme von $\mathrm{T}_{G}$ mit zunehmender Spannung zu erkennen. Die höchste Glasübergangstemperatur wurde bei einer äußeren Spannung von 1 MPa mit $643 \mathrm{~K}$ bestimmt. Bei der größten verwendeten Spannung (10,8 MPa) konnte nur noch eine Temperatur von $622 \mathrm{~K}$ für $\mathrm{T}_{G}$ beobachtet werden. Mit der Annahme eines linearen Zusammenhanges kann somit eine Abnahme der Glasübergangstemperatur von 2,35 K pro MPa berechnet werden.

\section{Untersuchung des Aktivierungsvolumens}

Ebenfalls wurde wie bei den Messungen zu PdCuSi das Aktivierungsvolumen von $\mathrm{ZrAlCu}$ bestimmt. Hierfür wurde nur der nichtlineare Bereich, in dem das Material dehnungsratensensitiv ist, verwendet. Ein beispielhafter Verlauf ist in Abbildung 3.36 im Temperaturbereich von $610 \mathrm{~K}$ bis $680 \mathrm{~K}$ zu sehen. Es wurden dabei die Kräfte $500 \mathrm{mN}$ (4,9 $\mathrm{MPa}$ - schwarz) und $100 \mathrm{mN}$ (1,0 MPa - rot) verwendet. Eine Überlappung der Aktivierungsvolumina ist im Bereich bis $620 \mathrm{~K}$ zu erkennen, wobei die 
Volumina leicht ansteigen (bis 17,73 $\mathrm{nm}^{3}$ - entspricht einer Atommenge von 980 Atomen). Bei höheren Temperaturen laufen die Kurven auseinander, ein Maximum für die größere Kraft ist bei $628 \mathrm{~K}\left(17,80 \mathrm{~nm}^{3}\right.$ - 990 Atomen) und für die kleinere bei 638 K (18,20 nm³ - 1010 Atomen) zu sehen. Nachfolgend werden die Aktivierungsvolumia für beide Kräfte kleiner, wobei sie die größte Differenz in der unterkühlten Schmelze bei $644 \mathrm{~K}$ - $648 \mathrm{~K}$ mit 0,70 nm³ (390 Atomen) aufweisen. Ein Minimum durchlaufen beide Kurven bei $666 \mathrm{~K}$, mit einer Volumendifferenz von 0,31 $\mathrm{nm}^{3}$ - 20 Atome $\left(15,31 \mathrm{~nm}^{3}-100 \mathrm{mN}\right.$ zu 15,00 nm³ - $\left.500 \mathrm{mN}\right)$. Bei höheren Temperaturen steigen die Aktivierungsvolumina wieder an, eine erneute Überlappung kann jedoch erst bei deutlich höheren Temperaturen ( $\mathrm{T}>700 \mathrm{~K}$ ) beobachtet werden.

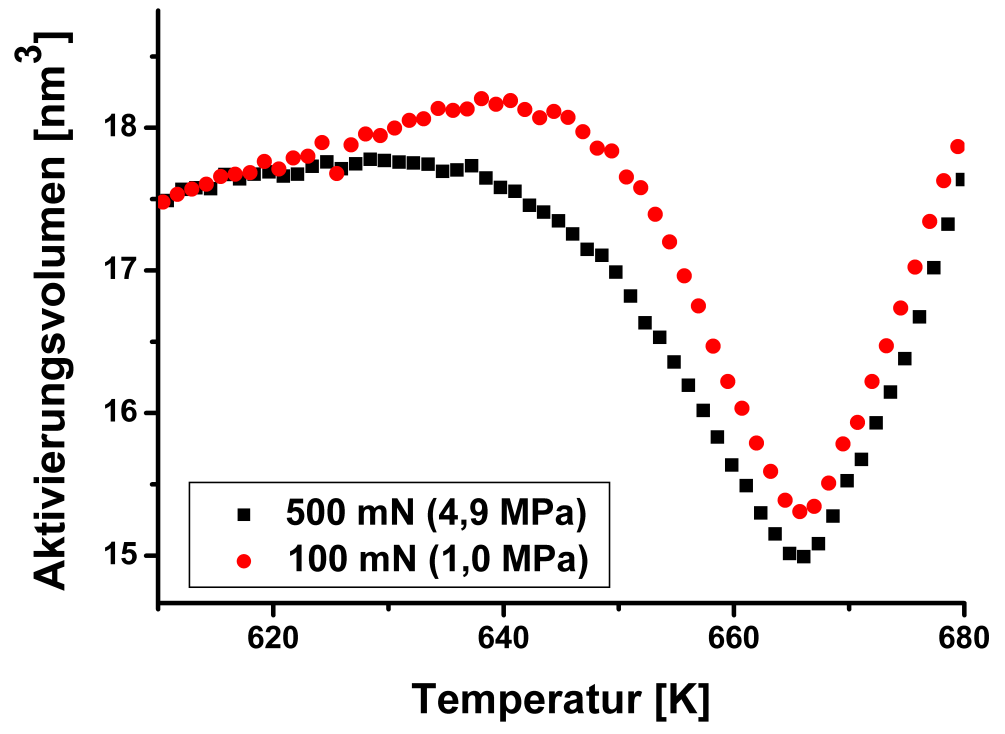

Abbildung 3.36: Aktivierungsvolumen von $\mathrm{ZrAlCu}$ im Temperaturbereich von $610 \mathrm{~K}$ bis $680 \mathrm{~K}$ bei den verwendeten Kräften $500 \mathrm{mN}$ (4,9 MPa - schwarz) und $100 \mathrm{mN}(1,0 \mathrm{MPa}-\mathrm{rot})$

Bei Auswertung von weiteren Messungen mit unterschiedlichen Kräften kann wie beim PdCuSi (siehe Abb. 3.29) ein spannungsabhängiges Aktivierungsvolumen beobachtet werden. In Abbildung 3.37 sind sowohl die maximal erhaltenen Aktivierungsvolumen als auch die zugehörigen Temperaturen in Abhängigkeit der äußeren Spannung aufgetragen. Übersichtshalber wurden die Werte ebenfalls als rote Punkte in der x-y-Ebene dargestellt. Es ist sowohl eine Abnahme des Aktivierungsvolumens als auch der Temperatur (Um- 
kehrpunkt in der Kurve) mit Erhöhung der äußeren Spannung zu erkennen. Dabei ist eine Reduzierung um 9,10 $\mathrm{nm}^{3}$ (500 Atome) von 18,21 nm³ (1010 Atome) auf 9,11 nm (510 Atome) zu beobachten, wobei die äußere Spannung von $1 \mathrm{MPa}$ auf $16 \mathrm{MPa}$ erhöht wurde. Größere Kräfte konnten aufgrund des Messgerätes nicht untersucht werden. In der Temperatur kann eine Abnahme von 19 K (644 K zu $625 \mathrm{~K}$ ) bestimmt werden.

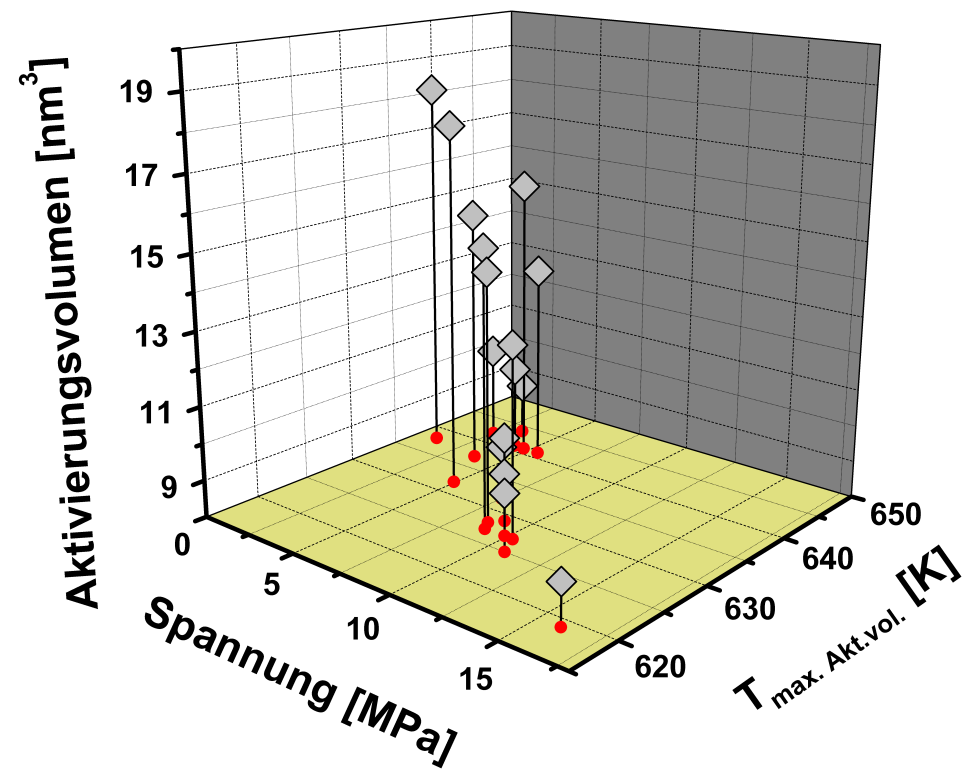

Abbildung 3.37: Maximal bestimmtes Aktivierungsvolumen von $\mathrm{ZrAlCu}$ und die zugehörige Temperatur in Abhängigkeit der äußeren Spannung

Um das Verhalten des Aktivierungsvolumens auch beim $\mathrm{ZrAlCu}$ in der unterkühlten Schmelze in Abhängigkeit der Dehnung untersuchen zu können, wird, wie im Kapitel 3.1.4, die Dehnungsrate im Bereich der plastischen Deformation (in der Nähe von $\mathrm{T}_{G}$ ) bei konstanter Dehnung bei drei verschiedenen äußeren Spannungen (5,0 MPa, 10,9 MPa und 13,7 MPa) beobachtet. Dazu wurde, wie im Kapitel 1.3.1.2 (Gleichung 1.12) beschrieben, die wahre Dehnung berechnet und gegenüber einer berechneten Dehnungsrate (Kapitel 2.1.4, Gleichung 2.1) aufgetragen (siehe Abb. 3.38). Die so erhaltenen Dehnungsraten wurden halblogarithmisch gegenüber den wahren Spannungen aufgetragen. Unter der Annahme, dass ein lineares Verhalten vorliegt, kann für jede betrachtete Dehnung eine Steigung bestimmt werden, welche der Berechnung des Aktivierungsvolumens dient. Hierfür wurde Gleichung 1.14 unter der Annahme verwendet, dass die Temperatur konstant $\mathrm{T}_{G}(640 \mathrm{~K})$ betrug. 
Um sicherzustellen, dass für diese Auswertung nur der nichtlineare, plastische Dehnungsbereich des Materials betrachtet wurde, wurden nur Dehnungen von über einem Prozent verwendet. Wie in Abbildung 3.33 zu erkennen ist, ist somit gewährleistet, dass nur der Bereich in der unterkühlten Schmelze verwendet wird. Bei Dehnungen über 2,5\% besteht jedoch die Gefahr, dass das Material anfängt zu kristallisieren. Dies ist an den bestimmten Steigungen aus Abbildung 3.38 ebenfalls zu erkennen. Dort ist die halblogarithmische Auftragung und in Tabelle 3.2 die zugehörigen Steigungen m zu sehen. Es ist zu erkennen, dass die Steigungen mit zunehmender Dehnung bis 2,5 \% abnehmen, anschließend jedoch wieder größer werden. Dabei wurde bei der kleinsten Dehnung (1,5 \%) eine Steigung von 2,90 $\mathrm{MPa}^{-1}$ bestimmt. Die geringste Steigung mit 1,84 $\mathrm{MPa}^{-1}$ wurde bei 2,5\% berechnet.

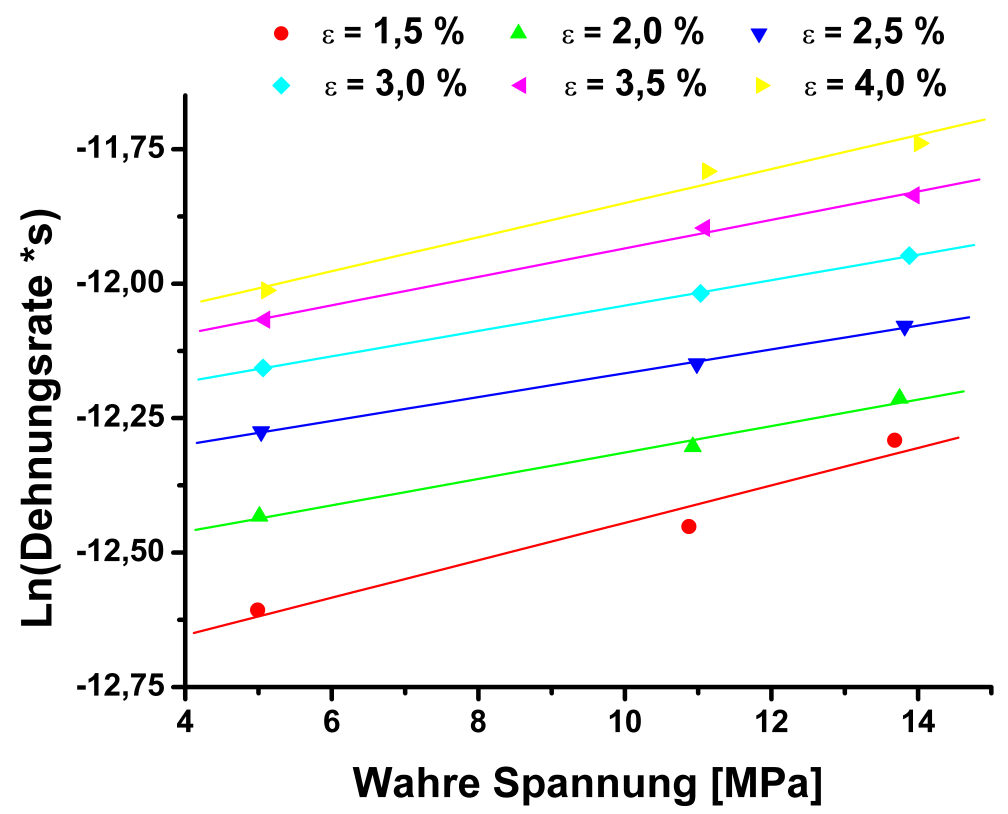

Abbildung 3.38: Halblogarithmische Auftragung der Dehnungsrate bei konstanter Dehnung gegenüber der wahren Spannung für ZrAlCu Proben

Die so erhaltenen Werte wurden direkt zur Bestimmung des Aktivierungsvolumens verwendet, welches in Abbildung 3.39 in Abhängigkeit der Dehnung aufgetragen wurde. Es ist eine exponentielle Abnahme des Aktivierungsvolumens mit zunehmender Dehnung bis 2,5 \% zu erkennen. Bei einer Dehnung von 1,5\% wurde ein Volumen von $25,63 \mathrm{~nm}^{3}$ (1430 Atome) bestimmt, welches bis 2,5\% auf 16,26 $\mathrm{nm}^{3}$ (900 Atome) abnimmt. Anschließend nimmt das Aktivierungsvolumen wieder zu, wobei ein anderes Verhalten als bei der Abnahme zu erkennen ist. Bei $4 \%$ Dehnung kann so ein Volumen 
von 21,65 nm³ (1200 Atome) berechnet werden. In der Abbildung wurde für das Aktivierungsvolumen bis 2,5\% ein exponentielles Verhalten (schwarze Kurve) angenommen, bei größerer Dehnung scheint das Volumen wieder linear anzusteigen. Der Wendepunkt des Verhaltens (etwa 2,7 \% Dehnung, vergleiche auch Abb. 3.33) wurde mit einem Pfeil und Kristallisation gekennzeichnet.

\begin{tabular}{|c|c|}
\hline$\epsilon[\%]$ & $\mathrm{m}\left[\mathrm{MPa}^{-1}\right]$ \\
\hline 1,5 & 2,90 \\
\hline 2,0 & 2,05 \\
\hline 2,5 & 1,84 \\
\hline 3,0 & 1,96 \\
\hline 3,5 & 2,20 \\
\hline 4,0 & 2,45 \\
\hline
\end{tabular}

Tabelle 3.2: Bestimmte Steigungen zur Berechnung des Aktivierungsvolumens aus der Auftragung in Abbildung 3.38

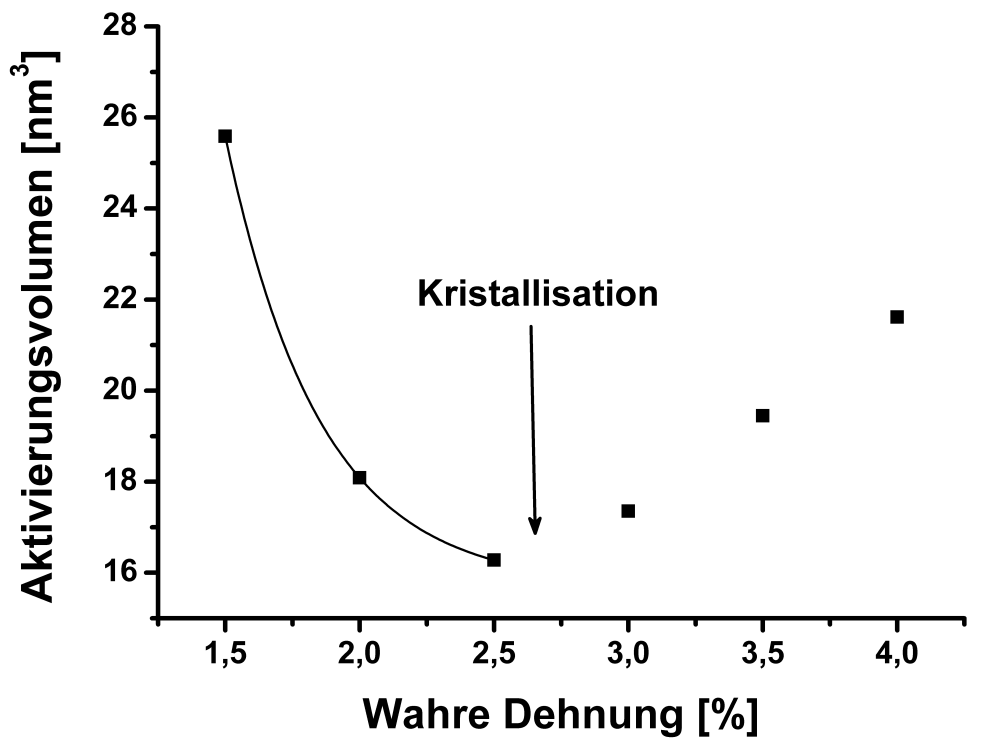

Abbildung 3.39: Aktivierungsvolumen in Abhängigkeit der wahren Dehnung in der unterkühlten Schmelze von $\mathrm{ZrAlCu}$

Für den Bereich vor der Kristallisation (Dehnungen $<2,7 \%$ ) kann analog zu den Messungen an PdCuSi unter Verwendung der Gleichung $3.2\left(\right.$ mit $\Omega_{\infty}=15,65 \mathrm{~nm}^{3}$, 
$\mathrm{A}=661,5 \mathrm{~nm}^{3}$ und $\left.\tau=0,3576\right)$ ein Aktivierungsvolumen für ein HochtemperaturBarrierenlimit von $15,65 \mathrm{~nm}^{3}$ (870 Atome) abgeschätzt werden.

Es konnte somit wie im fragilen Glasbildner PdCuSi auch im starken $\mathrm{ZrAlCu}$ sowohl für die Glasübergangstemperatur als auch das Aktivierungsvolumen ein spannungsabhängiges Verhalten beobachtet werden, wobei ein deutlich größeres involviertes Volumen bestimmt wurde. Diese Ergebnisse werden im Kapitel 4 ausführlich diskutiert. 


\subsection{Relaxationsprozesse von ABA-Block-Copolymeren}

Im zweiten Teil dieser Arbeit wurden Relaxationsprozesse in wasserstoffbrückenbildenden ABA-Block-Copolymeren untersucht (siehe Kapitel 2.2). Der Fokus lag dabei auf sekundären Relaxationen, welche unterhalb von $\mathrm{T}_{G}$ zu finden sind und durch ein Wasserstoffbrücken induziertes Chemical Confinement hervorgerufen werden, wie es Hachenberg et al. vorschlugen. ${ }^{[42]}$

a)

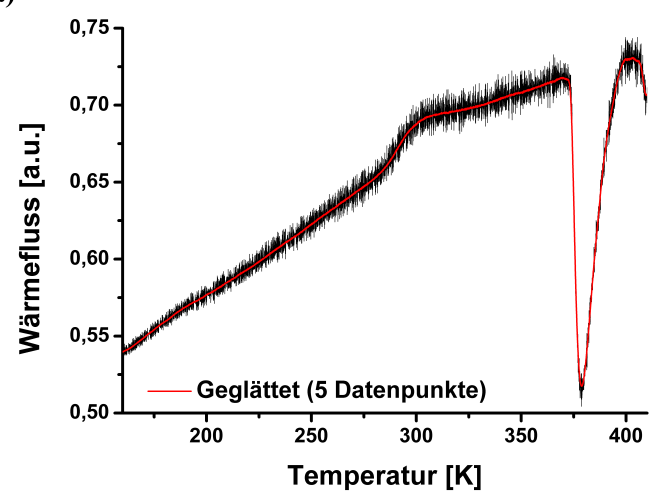

b)

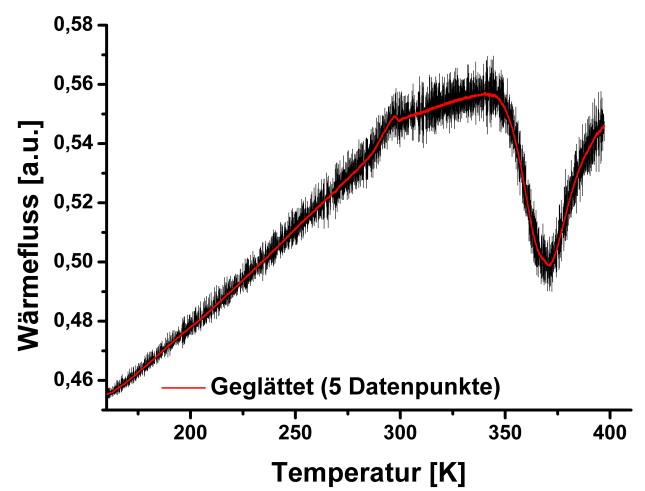

Abbildung 3.40: Vergleich von zwei DSC Messungen a) B-Block 5504g/mol, A-Block $11280 \mathrm{~g} / \mathrm{mol}$ und b) B-Block $9528 \mathrm{~g} / \mathrm{mol}$, A-Block $9390 \mathrm{~g} / \mathrm{mol}$.

Zur Charakterisierung der Glasübergangstemperatur der verwendeten ABA-BlockCopolymere und zum Vergleich zu den gemessenen Relaxationsspektren wurden DSC Messungen durchgeführt. In Abbildung 3.40 sind zwei Beispielspektren aufgeführt, wobei in Teilbild a) die Messkurve für ein Polymer mit einem inneren ptBA Block von $5504 \mathrm{~g} / \mathrm{mol}$ und äußeren Blöcken von $11280 \mathrm{~g} / \mathrm{mol}$ abgebildet ist. In schwarz sind die Messwerte des Wärmeflusses und in rot eine Glättung über 5 Datenpunkte zu sehen. Im Wärmefluss ist ein Ansteigen mit Erhöhung der Temperatur zu beobachten, wobei ein Knick in der Kurve bei $288 \mathrm{~K}\left(\mathrm{~T}_{G}\right)$ zu sehen ist. Bei höheren Temperaturen ist eine größere Steigung zu erkennen, welche ab $300 \mathrm{~K}$ wieder abflacht. Ein starkes Abknicken ist bei $370 \mathrm{~K} \mathrm{zu}$ beobachten, wobei der Wärmefluss deutlich abnimmt und somit ein exothermes Verhalten der Probe zu erkennen ist. Ein Minimum ist bei $379 \mathrm{~K}$ zu sehen, gefolgt von einem erneuten Ansteigen bei höheren Temperaturen.

Zum Vergleich ist im Bildteil b) die Messung eines Polymers mit einem inneren Block von $9528 \mathrm{~g} / \mathrm{mol}$ und äußeren Blöcken von $9390 \mathrm{~g} / \mathrm{mol}$ zu sehen. Der Verlauf des Wärmeflusses (Messpunkte - schwarz, Glättung über 5 Datenpunkte - rot) ist zu dem in Bildteil a) 
sehr ähnlich. Es ist ebenfalls ein leichtes Ansteigen unterhalb von $\mathrm{T}_{G}$ zu erkennen, wobei die Glasübergangstemperatur bei $286 \mathrm{~K}$ bestimmt werden konnte. Eine größere Steigung ist oberhalb von $\mathrm{T}_{G}$ zu sehen, wobei ein Überschießen bei $300 \mathrm{~K}$ zu erkennen ist, gefolgt von einem flacheren Ansteigen und einem starken exothermen Abknicken bei 343 K. Ein Minimum ist bei $372 \mathrm{~K}$ zu beobachten, welchem ein erneuter Anstieg im Wärmefluss folgt.

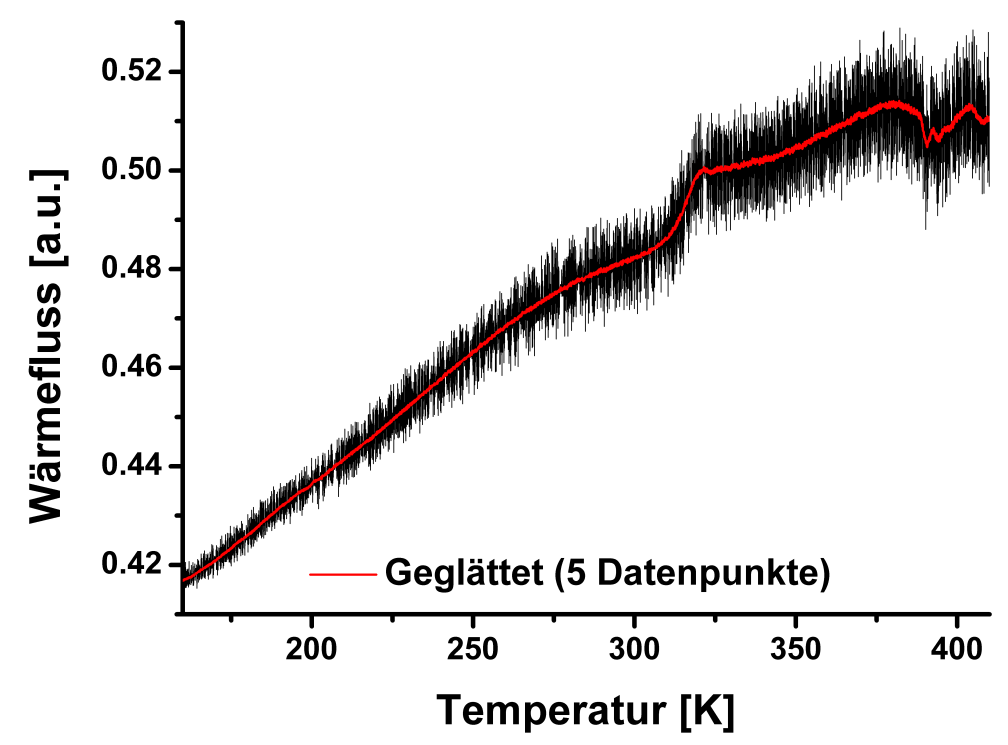

Abbildung 3.41: DSC Messung der Probe aus 3.40 b) nach Auslagerung bei $400 \mathrm{~K}$ für $30 \mathrm{~min}$.

Dasselbe Polymer (B-Block 9528 g/mol, A-Blöcke 9390 g/mol) wurde oberhalb der exothermen Reaktion bei $400 \mathrm{~K}$ für 30 min ausgelagert und erneut in der DSC vermessen (Messpunkte - schwarz, Glättung über 5 Datenpunkte - rot). Der erhaltene Wärmefluss ist in Abbildung $3.41 \mathrm{zu}$ sehen. Der Kurvenverlauf ist nur wenig abweichend von dem zuvor in Abbildung 3.40 beschriebenen, wobei nun jedoch die exotherme Reaktion bei über $350 \mathrm{~K}$ fehlt. Ein Ansteigen im Wärmefluss ist bis $\mathrm{T}_{G} \mathrm{zu}$ beobachten, wobei die Glasübergangstemperatur mit $308 \mathrm{~K}$ höher bestimmt wurde als bei der unbehandelten Probe. Oberhalb von $\mathrm{T}_{G}$ ist auch nur ein sehr geringes Abknicken zu erkennen (ab $382 \mathrm{~K}$ ), welches ebenfalls zu höheren Temperaturen verschoben ist.

Ein Vergleich der Glasübergangstemperaturen ist in Abbildung 3.42 dargestellt. Hierbei muss erwähnt werden, dass aufgrund eines schlechten Signal-Rausch-Verhältnisses 
und geringer Probenmengen weniger Proben mit DSC-Messungen ausgewertet wurden als anschließend über Relaxationsmessungen. Beim Vergleich der Glasübergangstemperaturen ist eine Abnahme um 6,5 K von 292,6 K (B-Block $3472 \mathrm{~g} / \mathrm{mol}$ ) auf 286,1 K (B-Block 9572 g/mol) zu sehen (schwarze Datenpunkte). Dies entspricht unter Annahme eines linearen Verhaltens (schwarze Gerade) einer Abnahme von $\mathrm{T}_{G}$ mit länger werdendem B-Block von 0,9 K pro $1000 \mathrm{~g} / \mathrm{mol}$. Zum Vergleich ist in rot der Messpunkt der Glasübergangstemperatur der ausgelagerten Probe zu sehen. Diese liegt bei $308 \mathrm{~K}$ um $22 \mathrm{~K}$ höher als bei der unbehandelten Probe.

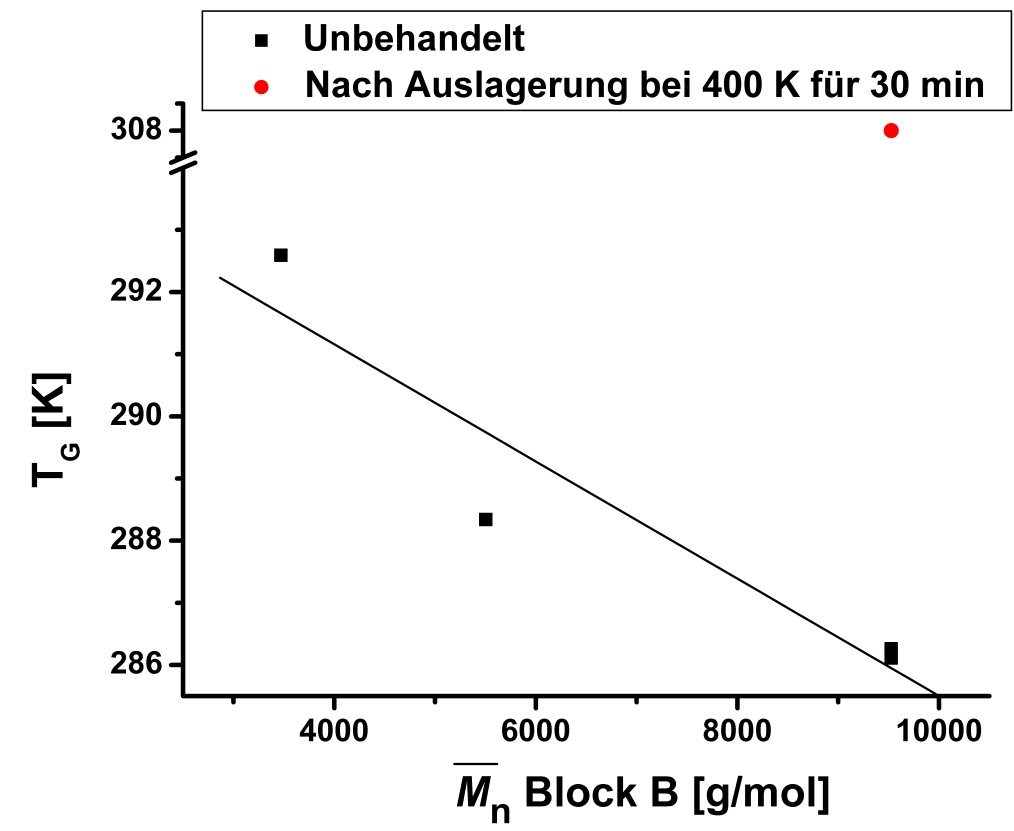

Abbildung 3.42: Glasübergangstemperatur aus DSC-Messungen bestimmt von unbehandelten Proben (schwarz) und einer bei $400 \mathrm{~K}$ für 30 min ausgelagerten Probe in Abhängigkeit des inneren B-Blockes.

Um diesen Effekt besser zu verstehen (siehe Kapitel 4), wurden Röntgenspektren von unbehandelten Proben mit der bei $400 \mathrm{~K}$ ausgelagerten verglichen. In Abbildung 3.43 ist eine Gegenüberstellung der Messungen zu sehen, bei der die erhaltenen Intensitäten gegenüber dem doppelten Reflektionswinkel im Bereich von $5^{\circ}$ bis $55^{\circ}$ dargestellt sind. Der Verlauf beider Kurven (schwarz - unbehandelt, rot - ausgelagert) ist sehr ähnlich, nur in der Intensität lassen sich bei der unbehandelten Probe höhere Werte erkennen. Nach einem kurzen Absinken der Intensität ist ein Maximum bei $9^{\circ} \mathrm{zu}$ sehen, gefolgt von einem großen Maximum bei $18^{\circ}$ und einem weiteren breiten Maximum bei $38^{\circ}$. Bei 
einem Winkel von über $40^{\circ}$ überlappen die Kurven und zeigen eine identische Intensität bis zum Abschluss der Messung.

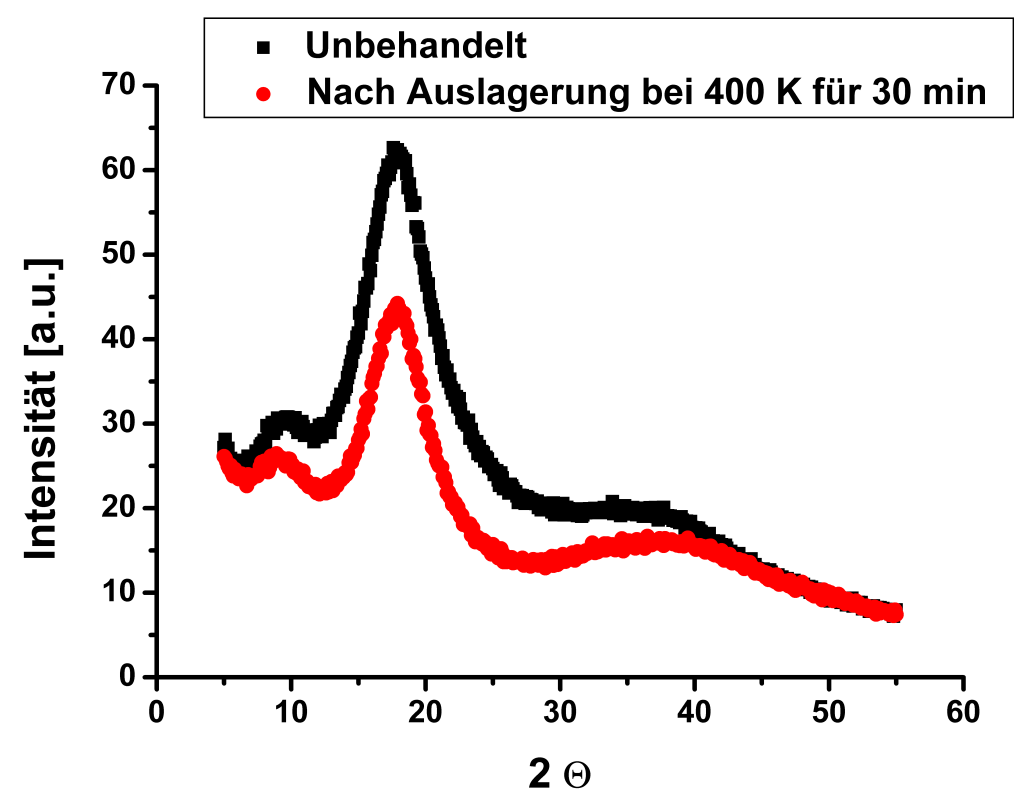

Abbildung 3.43: Vergleich der Röntgenspektren von ABA-Block-Copolymeren unbehandelt (schwarz) und nach Auslagerung für 30 min bei $400 \mathrm{~K}$ (rot).

Zur Untersuchung des Relaxationsverhaltens der ABA-Block-Copolymere wurden, wie im Kapitel 2.4 beschrieben, die Proben mit Hilfe einer DMA 7 vermessen. In Abbildung 3.44 sind beispielhaft drei Spektren dargestellt, in denen der Tangens des Phasenwinkels halblogarithmisch gegenüber der Probentemperatur aufgetragen wurde. In schwarz ist die Messkurve für das Polymer mit einem inneren Block von 1068 g/mol und äußeren Blöcken von $12600 \mathrm{~g} / \mathrm{mol}$, in rot mit $5505 \mathrm{~g} / \mathrm{mol}$ (innerer) und $11300 \mathrm{~g} / \mathrm{mol}$ (äußere) sowie in blau für $7055 \mathrm{~g} / \mathrm{mol}$ (innerer) und $12400 \mathrm{~g} / \mathrm{mol}$ (äußere) zu sehen. Unterhalb von $300 \mathrm{~K}$ können verschiedene Maxima ausgemacht werden, wobei unterhalb von $200 \mathrm{~K}$ $\gamma$ - Peaks unterschiedlicher Intensität zu beobachten sind. Der am stärksten ausgeprägte $\gamma$-Peak ist in der roten Kurve von $150 \mathrm{~K}$ bis $225 \mathrm{~K}$ zu sehen. Im Temperaturbereich von $200 \mathrm{~K}$ bis $300 \mathrm{~K}$ sind weitere Maxima zu erkennen, welche folgend in der Arbeit zugeordnet werden sollen.

In der Nähe der Raumtemperatur ist ein ausgeprägtes Maximum für alle Kurven zu beobachten, wobei dieses mit Zunahme des B-Blockes zu geringeren Temperaturen verschoben ist (324 K - schwarze Kurve, $314 \mathrm{~K}$ - rote Kurve, $308 \mathrm{~K}$ - blaue Kurve). Ein 
weiteres, stark ausgeprägtes Maximum ist bei Temperaturen oberhalb von $350 \mathrm{~K}$ zu sehen, wobei keine Zuordnung zu den einzelnen Blöcken bei diesen Messungen möglich ist.

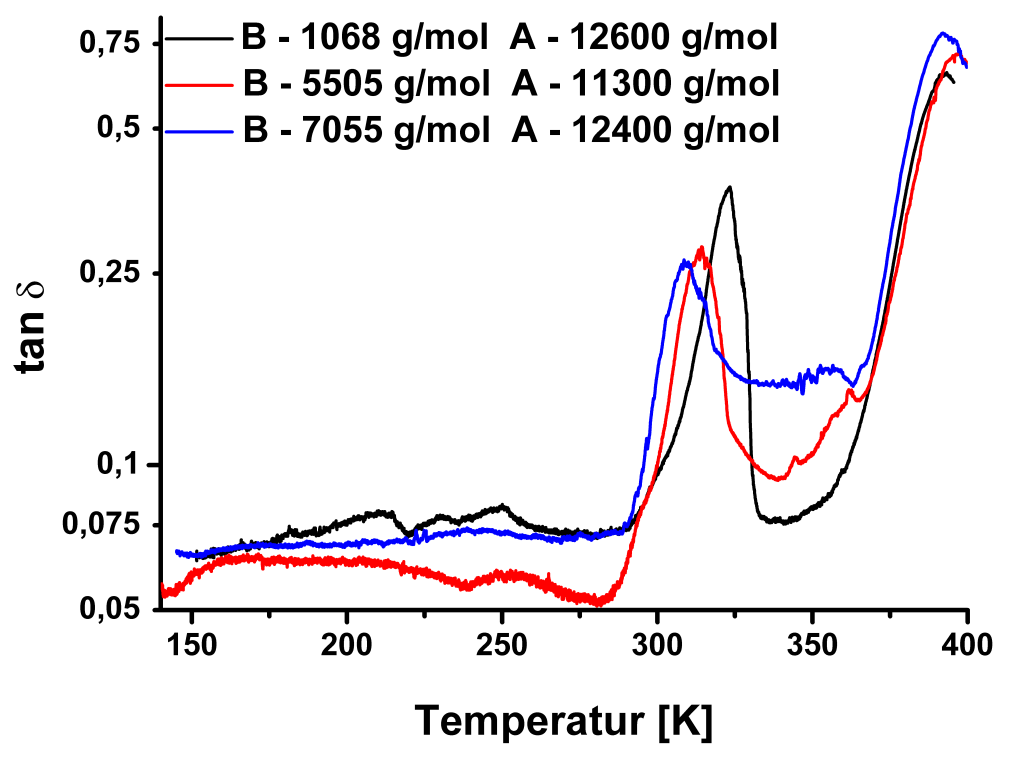

Abbildung 3.44: Vergleich der Relaxationsspektren von drei verschiedenen ABA-BlockCopolymeren (die Molekulargewichte der einzelnen Blöcke sind in der Abbildung genannt).

Ein Vergleich zwischen zwei Polymeren, bei denen beide denselben B-Block $(1068 \mathrm{~g} / \mathrm{mol})$ besaßen und sich nur in der Länge des A-Blockes unterschieden $(8200 \mathrm{~g} / \mathrm{mol}$-schwarz und $12600 \mathrm{~g} / \mathrm{mol}$ - rot), ist in Abbildung $3.45 \mathrm{zu}$ sehen. Dabei lässt sich unterhalb von $300 \mathrm{~K}$ nur bei 205 K (schwarz) und 209 K (rot) je ein Relaxationsmaximum erkennen, welches etwa derselben Temperatur zuzuordnen ist. Alle weiteren zu erkennenden Maxima treten nur jeweils bei den Einzelmessungen auf und sind nicht reproduzierbar. Bei $322 \mathrm{~K}$ ist zu erkennen, dass für beide Kurven das Maximum bei derselben Temperatur auftritt und mit der Glasübergangstemperatur aus den DSC-Messungen vergleichbar ist. Das Maximum bei Temperaturen über $350 \mathrm{~K}$ ist polymerabhängig verschoben, wobei für das Polymer mit dem kürzeren A-Block (schwarze Kurve) das Maximum früher (382 K) als bei dem Polymer mit längerem äußeren Block (roten Kurve - 393 K) zu beobachten ist. Ein Vergleich zwischen dem gemessenen Relaxationsspektrum und der Probenhöhe ist in Abbildung 3.46 zu sehen. Dabei wurde die Probenhöhe (Höhe des hergestellten 


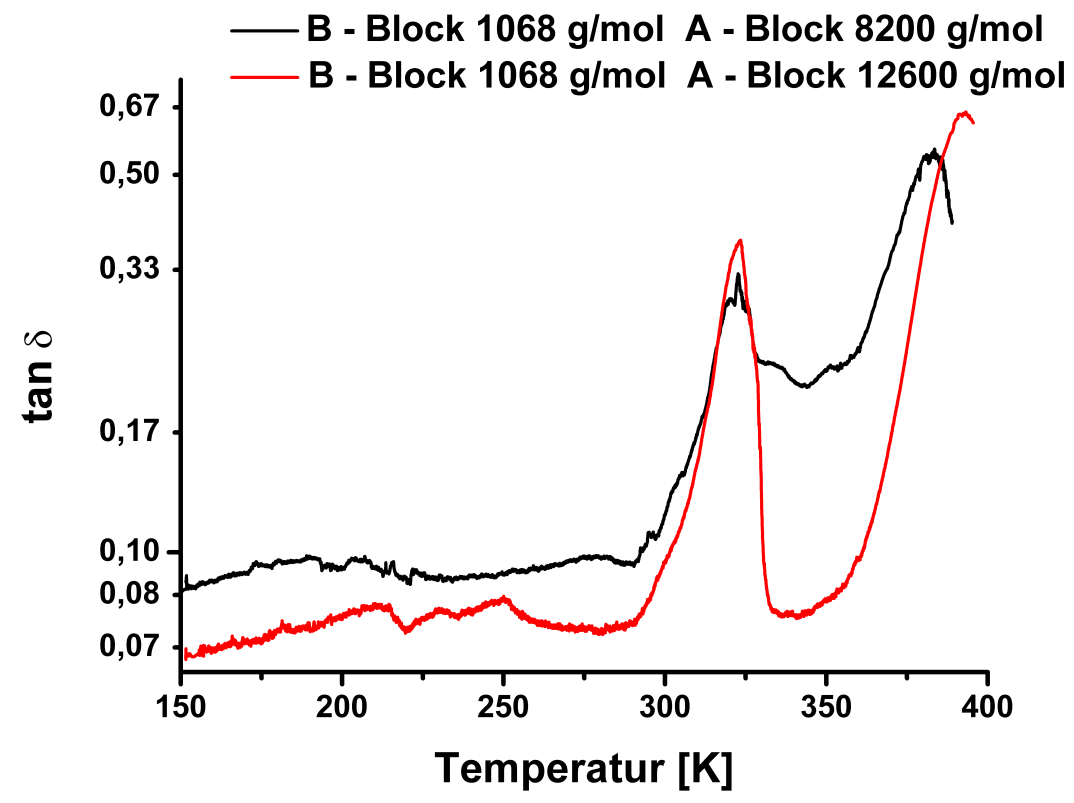

Abbildung 3.45: Vergleich des Relaxationspeaks bei $\mathrm{T}_{G}$ bei gleicher Länge des inneren Blockes B (die Molekulargewichte der einzelnen Blöcke sind in der Abbildung genannt).

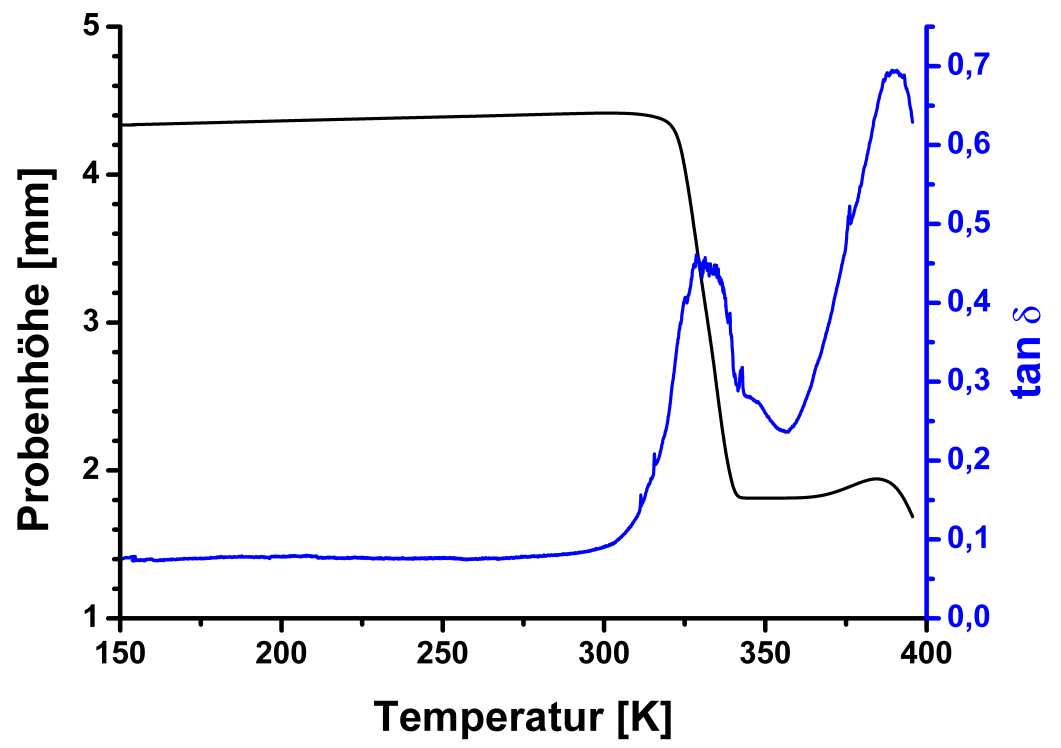

Abbildung 3.46: Vergleich der gemessenen Probenhöhe mit dem Relaxationsspektrum einer Polymerprobe mit einem B-Block von $3472 \mathrm{~g} / \mathrm{mol}$ und einem A-Block von $12800 \mathrm{~g} / \mathrm{mol}$. 
Polymerzylinders im Messtiegel - schwarze Kurve) genauso wie der Tangens des Phasenwinkels (blaue Kurve) gegenüber der Probentemperatur aufgetragen. Unterhalb von Raumtemperatur ist in der Probenhöhe ein Ansteigen mit sehr geringer Steigung zu erkennen, worauf bei $320 \mathrm{~K}$ ein starkes Abknicken zu beobachten ist $\left(\mathrm{T}_{G}\right)$, was auf ein Zusammendrücken der Probe und ein Ausfüllen des Probenbehälters zurückzuführen ist. Hierbei fällt der Wendepunkt der Kurve mit dem stark ausgeprägten Relaxationspeak bei Raumtemperatur zusammen. Anschließend ist in der Probenhöhe ein konstantes Verhalten von $340 \mathrm{~K}$ bis $360 \mathrm{~K}$ zu beobachten, worauf sich bei höheren Temperaturen das Polymer ausdehnt und ab $385 \mathrm{~K}$ aus der Probenhalterung herausgedrückt wird (erneute Abnahme in der Höhe). Hierbei ist zu erkennen, dass der zweite Relaxationspeak (Maximum bei $390 \mathrm{~K}$ ) im selben Temperaturbereich zu finden ist.

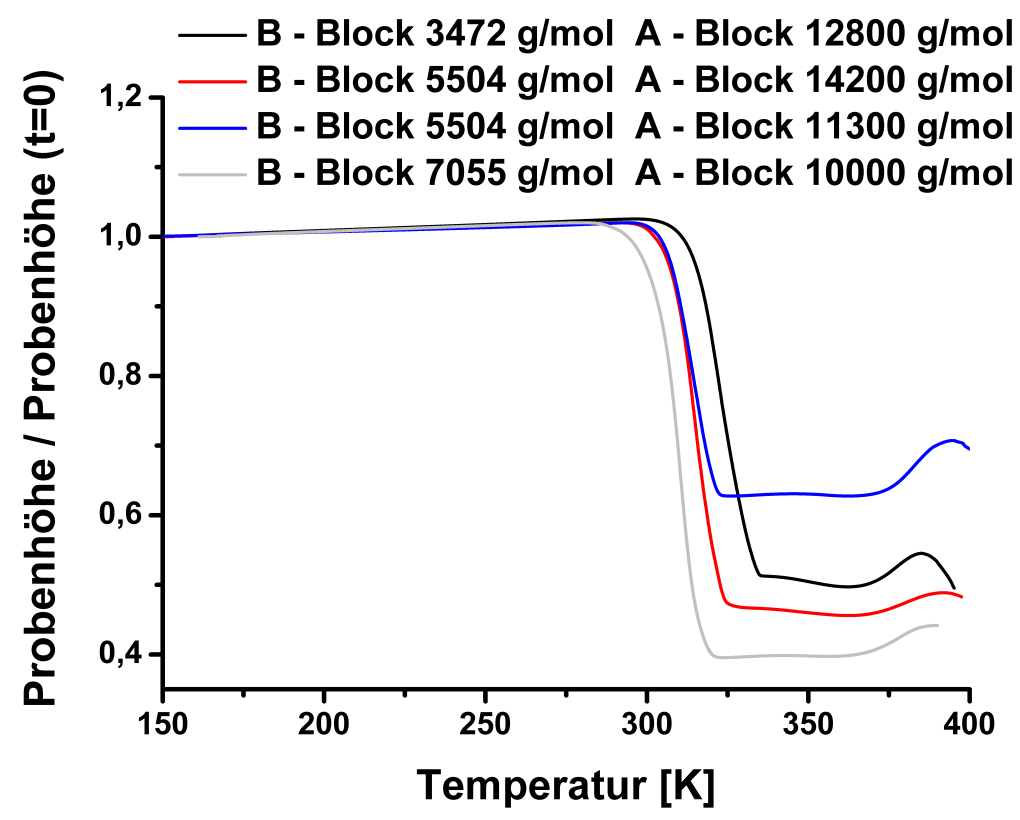

Abbildung 3.47: Vergleich der gemessenen Probenhöhe von vier verschiedenen ABABlock-Copolymeren (die Molekulargewichte der einzelnen Blöcke sind in der Abbildung genannt).

Ein Vergleich der Probenhöhen am Beispiel von vier verschiedenen Polymeren mit drei verschiedenen B-Blöcken (schwarze Kurve - B 3472 g/mol, A 12800 g/mol, rote Kurve B 5504 g/mol, A 14200 g/mol, blaue Kurve B 5504 g/mol, A 11300 g/mol und graue Kurve B $7055 \mathrm{~g} / \mathrm{mol}$ und A $10000 \mathrm{~g} / \mathrm{mol}$ ) ist in Abbildung $3.47 \mathrm{zu}$ sehen. Es wurde die Probenhöhe auf den Anfangswert normiert. Alle Kurven verlaufen bis $\mathrm{T}_{G}$ überlappend 
mit geringer Steigung bei Erhöhung der Temperatur. Ein Abknicken ist bei allen Kurven zu erkennen, wobei sich die Glasübergangstemperatur zu geringeren Temperaturen mit längerem B-Block verschiebt $\left(\mathrm{T}_{G}\right.$ bestimmt über Onset-Methode, $7055 \mathrm{~g} / \mathrm{mol}-300 \mathrm{~K}$, $5504 \mathrm{~g} / \mathrm{mol}-307 \mathrm{~K}$ und $3472 \mathrm{~g} / \mathrm{mol}$ - $315 \mathrm{~K}$ ). Hierbei fällt auf, dass sowohl die blaue als auch die rote Kurve aufgrund des gleichlangen inneren Blockes (5504 g/mol) einen sehr vergleichbaren Kurvenverlauf bis hin zu $\mathrm{T}_{G}$ aufweisen. Ebenfalls ist nach dem Ausfüllen des Probenbehälters ein konstantes Verhalten in allen Messungen zu beobachten, wobei der Temperaturbereich abhängig von der Probengröße (größere Proben brauchen eine längere Zeit, um komplett zusammengedrückt zu werden) zwischen $15 \mathrm{~K}$ und $30 \mathrm{~K}$ schwankt. Das erneute Ausdehnen der Proben ist abhängig von den Längen der A-Blöcke zu beobachten. So ist beim längsten A-Block (14200 g/mol - rot) der Onset in der Probenposition bei $374 \mathrm{~K}$ zu sehen, beim kürzesten (10000 g/mol - grau) schon bei $370 \mathrm{~K}$.

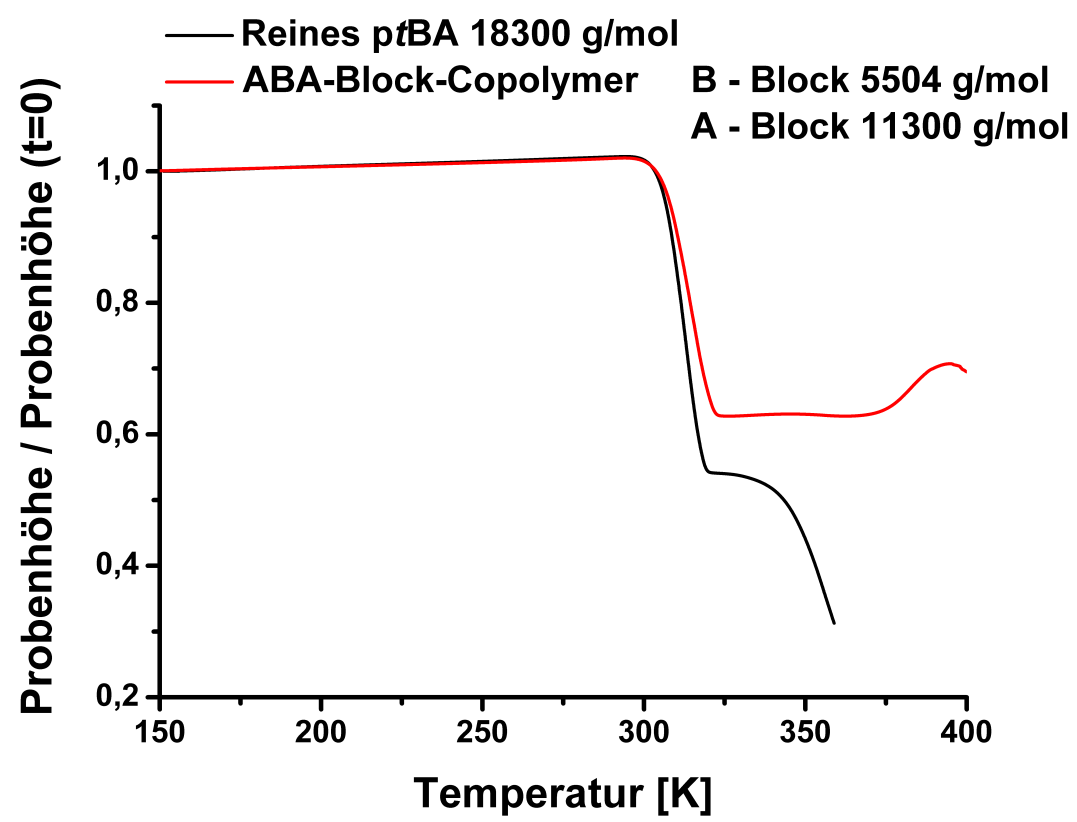

Abbildung 3.48: Vergleich der gemessenen Probenhöhe eines ABA-Block-Copolymers mit einer reinen $\mathrm{p} t \mathrm{BA}$ Probe (die Molekulargewichte der einzelnen Blöcke sind in der Abbildung genannt).

In Abbildung 3.48 ist ein Vergleich zwischen einem ABA-Block-Copolymer (rot, B $5504 \mathrm{~g} / \mathrm{mol}$, A $11300 \mathrm{~g} / \mathrm{mol}$ ) und einem Polymer aus reinem ptBA (schwarz, $18300 \mathrm{~g} / \mathrm{mol}$, entspricht dem Polymer des inneren Blockes) zu sehen. Es konnte 
eine in etwa gleiche Glasübergangstemperatur (305 K) beobachtet werden, wobei beim reinen $\mathrm{p} t \mathrm{BA}$ ein steilerer Abfall und somit ein schnelleres Zusammendrücken des Polymers erkennbar ist. Beim ABA-Block-Copolymer ist bis zur erneuten Ausdehnung bei $370 \mathrm{~K}$ ein konstantes Verhalten und das Herausquellen aus dem Behältnis ab $395 \mathrm{~K}$ zu sehen. Das reine ptBA zeigt jedoch ein anderes Verhalten. Nach dem Zusammendrücken und Ausfüllen des Behälters ist ein sofortiges Herausdrücken des Polymers zu erkennen, weshalb die Messung auch schon bei $360 \mathrm{~K}$ beendet werden musste. Ein konstantes Verhalten oberhalb von $\mathrm{T}_{G}$ und eine erneute Ausdehnung des Polymers konnte nicht beobachtet werden.

a)

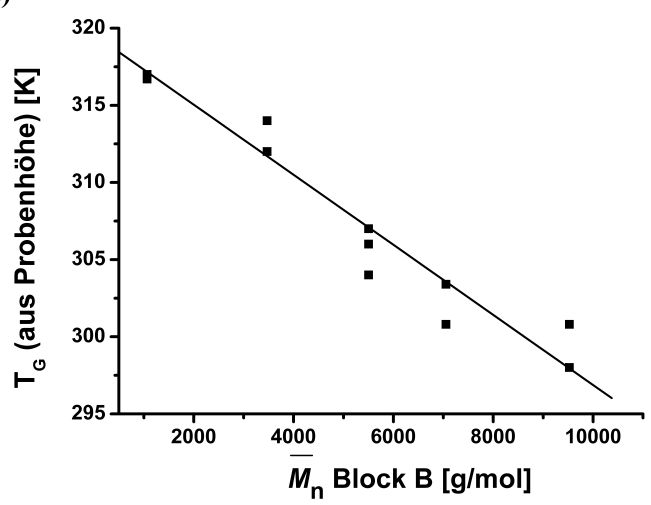

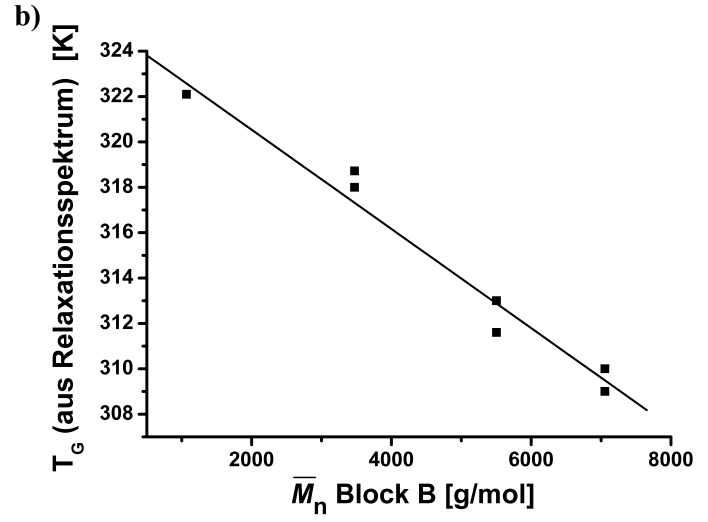

Abbildung 3.49: Vergleich der Glasübergangstemperatur bestimmt aus der gemessenen Probenhöhe a) und den Relaxationsspektren b) in Abhängigkeit des inneren B-Blockes

Die aus diesen Messungen gewonnenen Daten für die Glasübergangstemperatur sind in Abbildung 3.49 zu sehen. Dabei ist $\mathrm{T}_{G}$ im Bildteil a) als Onset in der Probenhöhe und im Bildteil b) als das Maximum des Relaxationspeaks bei Raumtemperatur definiert und jeweils gegen das Molgewicht des inneren Blockes aufgetragen. Mit beiden Methoden lässt sich ein linearer Zusammenhang (schwarze Geraden) zur Länge des B-Blockes nachweisen. Im Bildteil a) ist eine Abnahme von $\mathrm{T}_{G}$ um $19 \mathrm{~K}$ von $317 \mathrm{~K}(1068 \mathrm{~g} / \mathrm{mol})$ auf $298 \mathrm{~K}(9528 \mathrm{~g} / \mathrm{mol})$ zu erkennen. Dies entspricht einer Abnahme von 2,3 \pm 0,2 K pro $1000 \mathrm{~g} / \mathrm{mol}$ Zunahme im inneren Block. Im Bildteil b) nimmt die Glasübergangstemperatur um $13 \mathrm{~K}$ von $322 \mathrm{~K}(1068 \mathrm{~g} / \mathrm{mol})$ auf $309 \mathrm{~K}(7055 \mathrm{~g} / \mathrm{mol}) \mathrm{ab}$, was einer Abnahme von 2,2 \pm 0,1 K pro $1000 \mathrm{~g} / \mathrm{mol}$ Zunahme in Block B entspricht. In Abbildung 3.50 wurde die Temperatur des Onsets der erneuten Probenausdehnung 
oberhalb von $\mathrm{T}_{G}$ gegenüber der Molekularmasse der äußeren Blöcke A aufgetragen. Es lässt sich ebenfalls ein linearer Zusammenhang (schwarze Gerade) erkennen, wobei die bestimmte Temperatur mit länger werdendem äußeren Block größer wird. Es ist eine Zunahme um 6 K von 368 K (8497 g/mol) auf 374 K (14184 g/mol) zu sehen. Bei einem linearen Zusammenhang entspricht dies einer Zunahme in der Temperatur des Onsets von 1,1 K pro $1000 \mathrm{~g} / \mathrm{mol}$ Zunahme des A-Blockes.

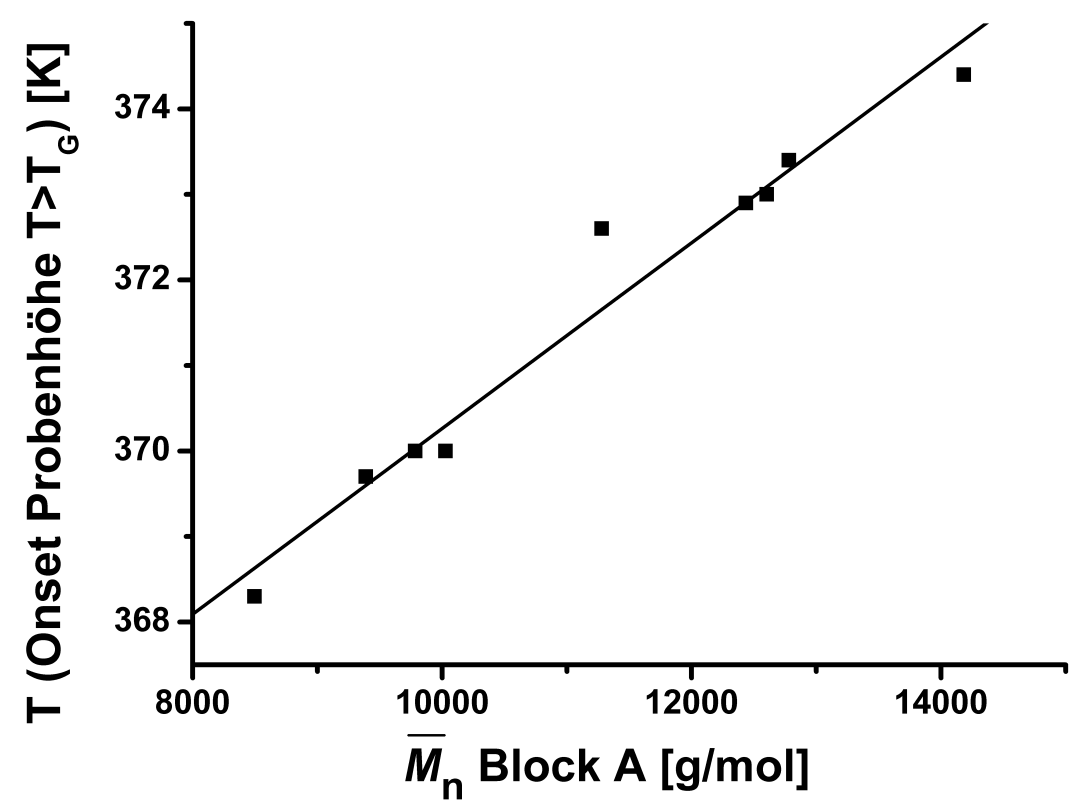

Abbildung 3.50: Vergleich der Änderung in der Probenposition oberhalb von $\mathrm{T}_{G}$ in Abhängigkeit der äußeren A-Blöcke.

Um auch eine Zuordnung der Relaxationsmaxima unterhalb von $300 \mathrm{~K}$ zu gewährleisten, wurden verschiedene Polymerproben mit denselben Längen der inneren Blöcke vermessen und miteinander verglichen. Ein Beispiel hierzu ist in Abbildung $3.51 \mathrm{zu}$ sehen. Es ist der Tangens des Phasenwinkels gegenüber der Probentemperatur im Bereich von $190 \mathrm{~K}$ bis $290 \mathrm{~K}$ zu sehen, wobei in rot die Messkurve des Polymers mit den Molgewichten von $5504 \mathrm{~g} / \mathrm{mol}$ (B-Block) und $11300 \mathrm{~g} / \mathrm{mol}$ (A-Block) und in orange mit $5504 \mathrm{~g} / \mathrm{mol}$ (B-Block) und $14200 \mathrm{~g} / \mathrm{mol}$ (A-Block) dargestellt wurden. In der roten Kurve ist bis 238 K ein Absinken des Phasenwinkels zu erkennen, gefolgt von einem Maximum bei $252 \mathrm{~K}$. In der orangen Kurve ist bis $206 \mathrm{~K}$ ein Absinken zu sehen, gefolgt von einem doppelten Maximum bei $218 \mathrm{~K}$ und $228 \mathrm{~K}$ und einem ausgeprägten Maximum bei $257 \mathrm{~K}$. 


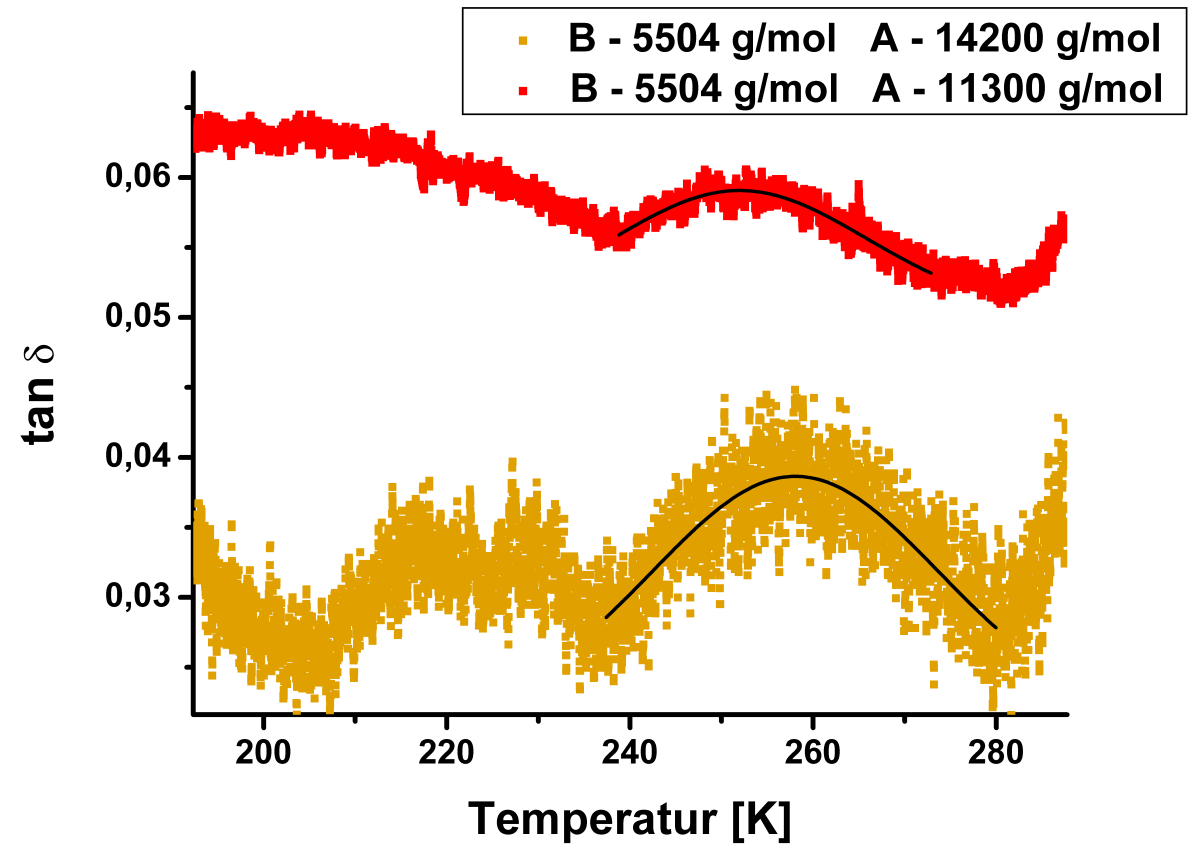

Abbildung 3.51: Vergleich der sekundären Relaxation unterhalb von $\mathrm{T}_{G}$ bei gleicher Länge des inneren Blockes B (die Molekulargewichte der einzelnen Blöcke sind in der Abbildung genannt).

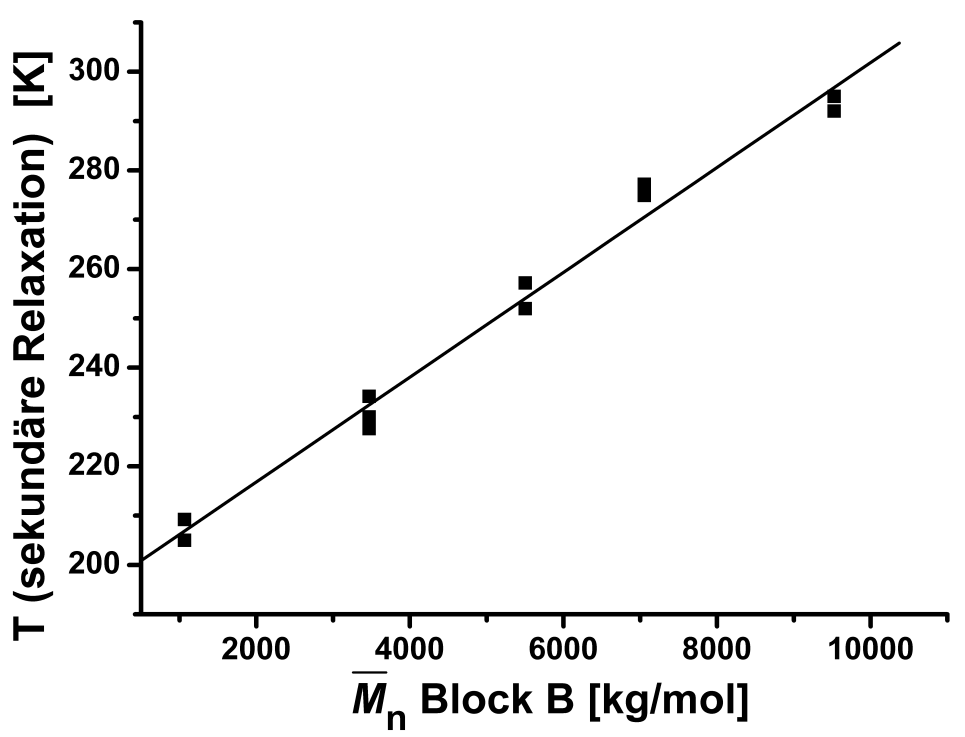

Abbildung 3.52: Temperatur des Maximums der sekundären Relaxation unterhalb von $\mathrm{T}_{G}$ in Abhängigkeit des inneren B-Blocks. 
Die Maxima bei 252 K (rote Kurve) und 257 K (orangene Kurve) wurden über ein GaußVerhalten $^{2}$ (schwarze Kurven) angepasst und die so erhaltenen Maximumtemperaturen von verschiedenen Messungen in Abhängigkeit des inneren Blockes B aufgetragen (siehe Abb. 3.52).

Es ist ein Anstieg in der Temperatur bei länger werdendem inneren Block zu erkennen. Dabei ist bei der sekundären Relaxation eine Zunahme um 87 K von 205 K (1068 g/mol) auf 292 K (9528 g/mol) zu beobachten. Bei der Annahme eines linearen Verhaltens (schwarze Gerade) bedeutet dies eine Verschiebung der Temperatur der sekundären Relaxation um 10,1 K pro $1000 \mathrm{~g} / \mathrm{mol}$ Zunahme des inneren Blockes.

2

$$
\delta=\delta_{0}+\frac{A}{w \cdot \sqrt{\frac{\pi}{2}}} \cdot e^{-2 \cdot\left(\frac{\bar{M}_{n}-\bar{M}_{n c}}{w}\right)^{2}}
$$

mit $\delta=$ Phasenwinkel, $\bar{M}_{n}=$ Molmasse, A,w $=$ Konstanten 


\section{Diskussion}

Relaxationsprozesse von komplexen Fluiden stellen einen bedeutenden Aspekt zum Verständnis sowohl von metallischen Gläsern als auch von Polymeren dar. Deshalb ist die Interpretation der makroskopischen Eigenschaften hinsichtlich ihrer mikroskopischen Vorgänge ein sehr wichtiges Thema in der aktuellen Forschung. In diesem Kapitel werden die vorgestellten Ergebnisse mit Hilfe der aktuellen Literatur interpretiert und eingeordnet. Es soll ein Einblick in die mikroskopischen Relaxationsvorgänge der untersuchten komplexen Fluide gegeben werden. Dabei wird der Fokus im ersten Abschnitt (Kapitel 4.1 - Relaxationen metallischer Gläser) auf der Interpretation hinsichtlich des Modells der potentiellen Energielandschaft (siehe Kapitel 1.4.1) liegen, um dieses somit weiter zu entwickeln und zu vertiefen.

Im zweiten Abschnitt (Kapitel 4.2 - Relaxationen von wasserstoffbrückenbildenden Block-Copolymeren) wird vor allem auf ein Wasserstoffbrücken-induziertes Chemical Confinement und die daraus resultierenden Relaxationsvorgänge eingegangen.

\subsection{Relaxationen metallischer Gläser}

\subsubsection{Heizratenabhängige Relaxationsprozesse von amorphem PdCuSi unterhalb der Glasübergangstemperatur}

Relaxationsvorgänge in Gläsern sind ein weit diskutiertes und untersuchtes Themengebiet. ${ }^{[37-40]}$ Es wurden bisher jedoch vor allem schnelle Heizraten von über $1 \mathrm{~K} / \mathrm{min}$ verwendet, um Phänomene wie den Glasübergang und das Merging der Relaxationsvorgänge untersuchen zu können. In dieser Arbeit wurde systematisch die Heizrate verringert, um Veränderungen in den mechanischen Eigenschaften zu studieren. Außerdem wurden Alterungsprozesse durch periodisches Aufheizen und Abkühlen einer Probe 
untersucht. Die Ergebnisse dieser Untersuchungen sind im Kapitel 3.1.1 abgebildet. Im Vergleich der Speichermodule bei verschiedenen Heizraten (Abb. 3.1 a) fällt auf, dass bei größeren Heizraten sowohl die Glasübergangstemperatur des Materials als auch die Kristallisationstemperatur zu höheren Temperaturen verschoben sind. Dies bedeutet, dass das Erweichen des Materials bei höherer Heizrate später stattfindet, was zunächst über den Zustand in der potentiellen Energielandschaft nicht erklärbar ist, weil alle Proben dieselbe thermische Vorgeschichte besitzen, da sie ohne weitere Behandlung nach der Herstellung verwendet wurden. Statistisch gesehen sollten sich die Proben deshalb in einem vergleichbaren Zustand innerhalb der PEL befinden. Die systematische Änderung der Glasübergangstemperatur ist in Abbildung $4.1 \mathrm{zu}$ sehen.

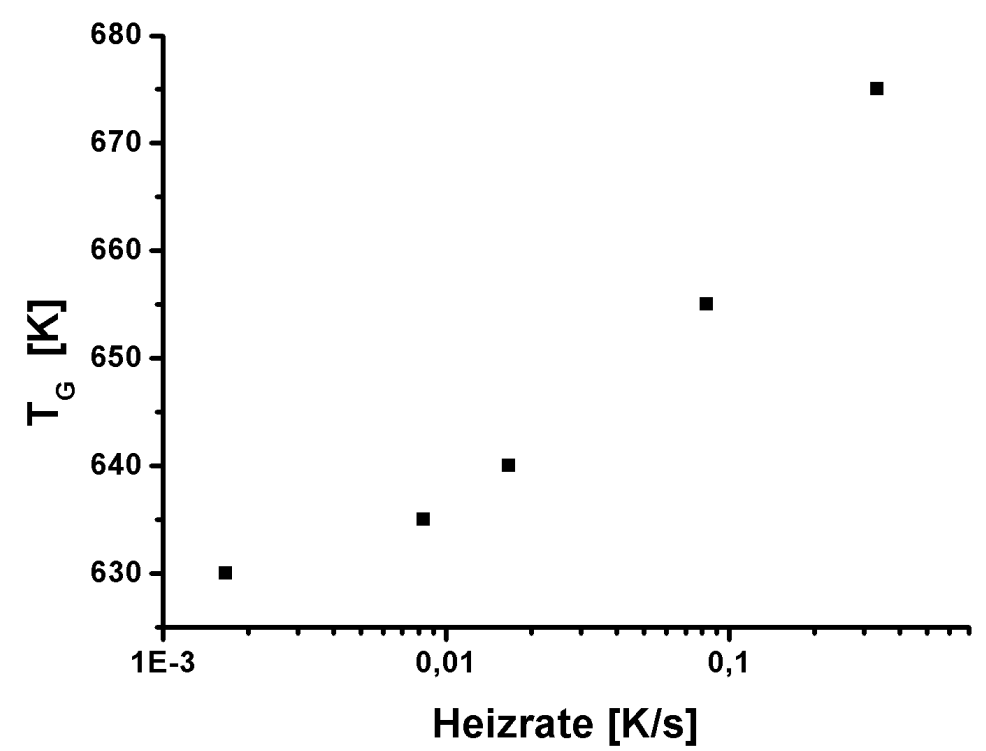

Abbildung 4.1: Abhängigkeit der Glasübergangstemperatur von der Heizrate

Dabei ist die Glasübergangstemperatur gegenüber der logarithmisch aufgetragenen inversen Heizrate dargestellt, wobei kein linearer Zusammenhang, sondern eine mit steigender Heizrate stärkere Verschiebung von $\mathrm{T}_{G}$ zu erkennen ist. In der Literatur wird dieses über die Kinetik der Gläser erklärt (siehe Kapitel 1.1.2), wobei von Brüning und Samwer für den Verlauf des $\alpha$ - Prozesses ein Vogel-Fulcher-Tammann-Verhalten vorgeschlagen wird. ${ }^{[39,90]}$

Im Vergleich dazu ist im Verlustmodul (siehe Abb. 3.1 b) eine starke Abhängigkeit in der Steigung des Wings zur Heizrate zu beobachten. In Abbildung 4.2 ist diese in 
Abhängigkeit zur inversen Heizrate aufgetragen. Es ist erkennbar, dass die Steigung mit zunehmender Heizrate kleiner wird. Mit dem im Kapitel 1.4.3 vorgestellten Modell lässt sich dieses Ergebnis als eine Anregung innerhalb der PEL erklären, da über die langsame $\beta$ - Relaxation energetisch höhere Subbasins innerhalb der PEL erreicht werden, was zur Folge hat, dass der Übergang in ein neues Metabasin schneller erreicht wird. Durch diesen Befund lässt sich auch die in der Literatur diskutierte (siehe Kapitel 1.1.1) heizratenabhängige Glasübergangstemperatur erklären.

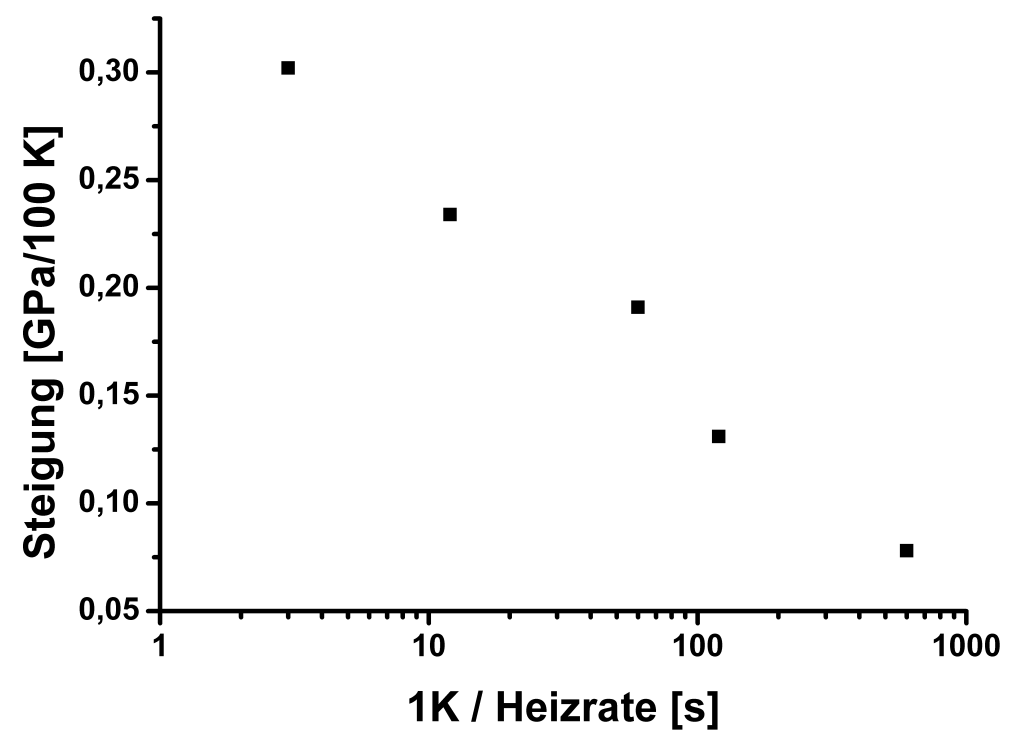

Abbildung 4.2: Abhängigkeit der Steigung des Wings von der Heizrate unterhalb der Glasübergangstemperatur

Durch die langsamere Heizrate können $\beta$ - Prozesse leichter aktiviert werden, so dass ein Übergang in die unterkühlte Schmelze bei niedrigeren Temperaturen möglich wird. Bei schnelleren Heizraten bewirkt die steifere Matrix jedoch eine Hemmung der kooperativen Prozesse. Dieser Effekt entspricht auch den Erwartungen, so beschreibt z. B. Kissinger, dass die Kinetik von thermisch aktivierten Prozessen heizratenabhängig ist. ${ }^{[91]}$ Somit ist anzunehmen, dass strukturelle Übergänge, wie sie im Bereich des Wings vermutet werden, ${ }^{[14,21,27,43]}$ bei langsamen Heizraten einfacher anregbar sind und somit einen breiteren Übergang zeigen, welcher sich in der geringeren Steigung des Wings wiederspiegelt.

Bei sehr langsamer Heizrate von 0,1 K/min konnte unterhalb der Glasübergangstemperatur ein zusätzliches Maximum im Verlustmodul bestimmt werden (siehe Abb. 3.2). 
Dieses überlagert zum Teil den Bereich des Wings, wobei dieser jedoch sichtbar bleibt. Das Dämpfungsmaximum ist bei Wiederholung der Messung (dieselbe Probe, wobei nicht über die Glasübergangstemperatur erwärmt wurde) reproduzierbar. Ab dem zweiten Erwärmen bleibt es konstant bei etwa $0,7 \mathrm{~T}_{G}$. Im Speichermodul ist im ersten Durchlauf ein sehr starker Anstieg des Realteils zu erkennen, welcher mit einer Alterung des Materials in Zusammenhang gebracht wird. ${ }^{[14,21,27]}$ Ab dem zweiten Durchlauf ist dieser nicht mehr reproduzierbar. Ebenfalls konnte im ersten Durchlauf, wie es auch Bedorf berichtete, ${ }^{[27]}$ ein Absinken im Verlustmodul beobachtet werden, welches auch einer Alterung, also einer Verringerung in der energetischen Positionshöhe innerhalb der PEL, zugeschrieben werden kann. Das zusätzliche Maximum kann jedoch keinen Alterungsprozess darstellen, da dieses reproduzierbar ist.

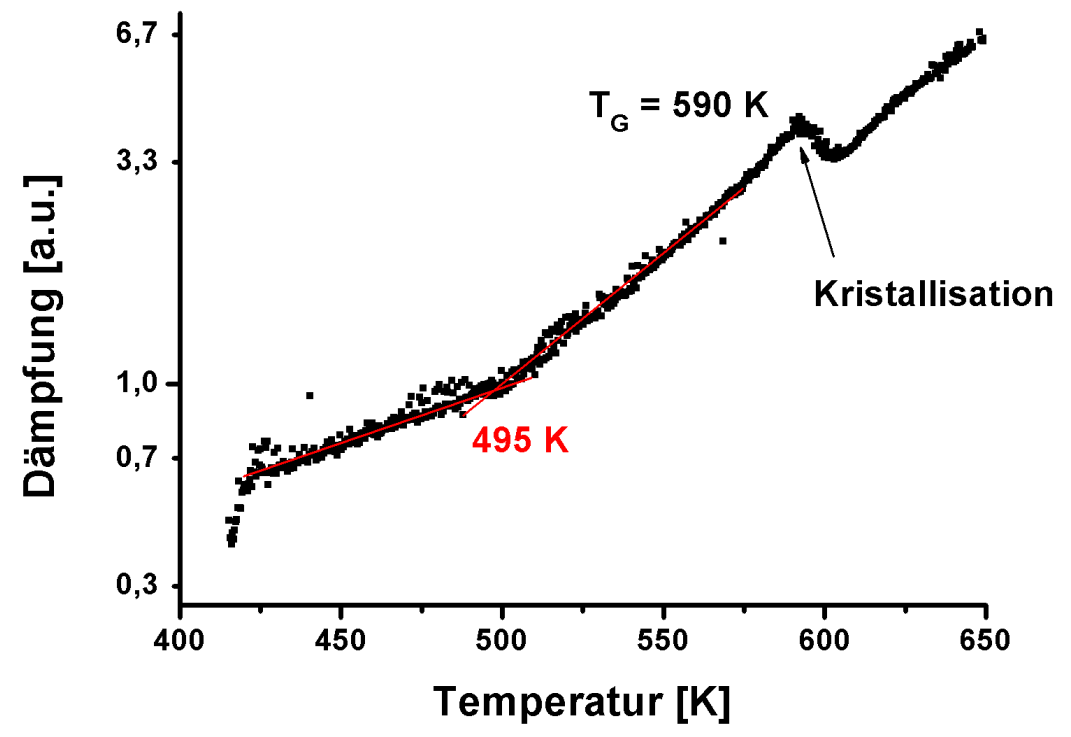

Abbildung 4.3: Dämpfungsmessung mittels DPO an einem amorphen $\operatorname{Pd}_{77,5} \mathrm{Cu}_{6,0} \mathrm{Si}_{16,5^{-}}$ Film mit einer Schichtdicke von $300 \mathrm{~nm}$, bei einer Frequenz von 4,6 kHz und einer Heizrate von 0,1 K/min. Der Schermodul liegt nahe Raumtemperatur bei 36 GPa. ${ }^{1}$

In einer vergleichenden DPO-Messung (siehe Kapitel 1.3.2.3) konnte bei einer Heizrate von ebenfalls $0,1 \mathrm{~K} / \mathrm{min}$ auch eine Änderung im Dämpfungsverhalten beobachtet werden. ${ }^{1}$ In Abbildung 4.3 ist die Dämpfung der PdCuSi Probe (Schichtdicke 300 nm,

\footnotetext{
${ }^{1}$ Messung von Dennis Bedorf, 1. Physikalisches Institut Georg-August-Universität zu Göttingen, 2010
} 
Frequenz 4,6 kHz) in Abhängigkeit der Probentemperatur aufgetragen. Bei $495 \mathrm{~K}$ ist ein Knick im Verlauf der Dämpfung sichtbar (etwa 0,8 $\mathrm{T}_{G}$, wobei die Glasübergangstemperatur durch die frühe Kristallisation nicht exakt bestimmbar ist), worauf der Verlust deutlich steiler ansteigt. Dies scheint ein Indiz dafür zu sein, dass die langsame $\beta$ - Relaxation nach dem Knick stärker angeregt ist. Es ist zu vermuten, dass ein struktureller Übergang solch ein Verhalten hervorrufen kann. Concustell et al. konnten ebenfalls ein zusätzliches Dämpfungsmaximum in Ultraschallexperimenten bei sehr langsamen Heizraten $\left(10^{-3} \mathrm{~K} / \mathrm{min}\right)$ feststellen, welches unterhalb der Glasübergangstemperatur auftrat, ${ }^{[92]}$ was zeigt, dass es sich um keine maschinell bedingte Relaxation handelt.

In Kolloid-Systemen wurde in Spannungs-Dehnungskurven bei ca. 30 \% unterhalb des Yielding Points solch ein struktureller Übergang beschrieben, welcher zur Folge hatte, dass Blockierungen im Material verringert und somit der nichtlineare Bereich leichter erreicht wurde. Dieser Übergang wurde als unabhängig von der Konfiguration des untersuchten Materials beschrieben. Durch Umgestaltungen der Atomabstände verändern sich die Bindungsverhältnisse der einzelnen Teilchen zueinander, so dass kooperative Bewegungen erleichtert werden. ${ }^{[93-96]}$ Bei der Annahme, dass Kolloide zum metallischen Glas starke Ähnlichkeiten zeigen, ${ }^{[97]}$ ist es möglich, dass auch in dem hier untersuchten Material solch ein Übergang stattfindet. Dabei sollte dieser viel Energie benötigen, da Bindungen aufgebrochen werden müssen, was auch in den Verlustmessungen durch das Maximum zu beobachten ist. So kann auch die beobachtete starke Alterung erklärt werden, da durch die leichtere Anregbarkeit von strukturellen Änderungen eine Relaxation in energetisch tiefer gelegenen Subbasins in der PEL erleichtert wird. Gestützt wird diese Hypothese über DSC-Messungen (siehe Abb. 3.5), da in dem beschriebenen Temperaturbereich bei einer verwendeten Heizrate von $0,1 \mathrm{~K} / \mathrm{min}$ ein starkes endothermes Ansteigen zu beobachten ist. Über einen langen Zeitraum von über 3 Stunden wird Energie aufgenommen, wobei es sich nicht um den Glasübergang handelt (bei etwa $600 \mathrm{~K}$ ), sondern einen zusätzlichen Prozess darstellt, der z. B. über einen wie oben dargestellten Übergang zu beschreiben ist.

Um die notwendigen Relaxationen bei dieser sehr langsamen Heizrate einzuordnen, wurden in Abbildung 4.4 heizratenabhängig die Temperaturen des einsetzenden $\alpha$ - Prozesses (der Onset (grün) in der Probenposition mit dem Beginn des Fließens der Proben) und des $\beta$ - Prozesses mit dem Einsetzen einer Alterung der Proben (Onset (rot) und der 


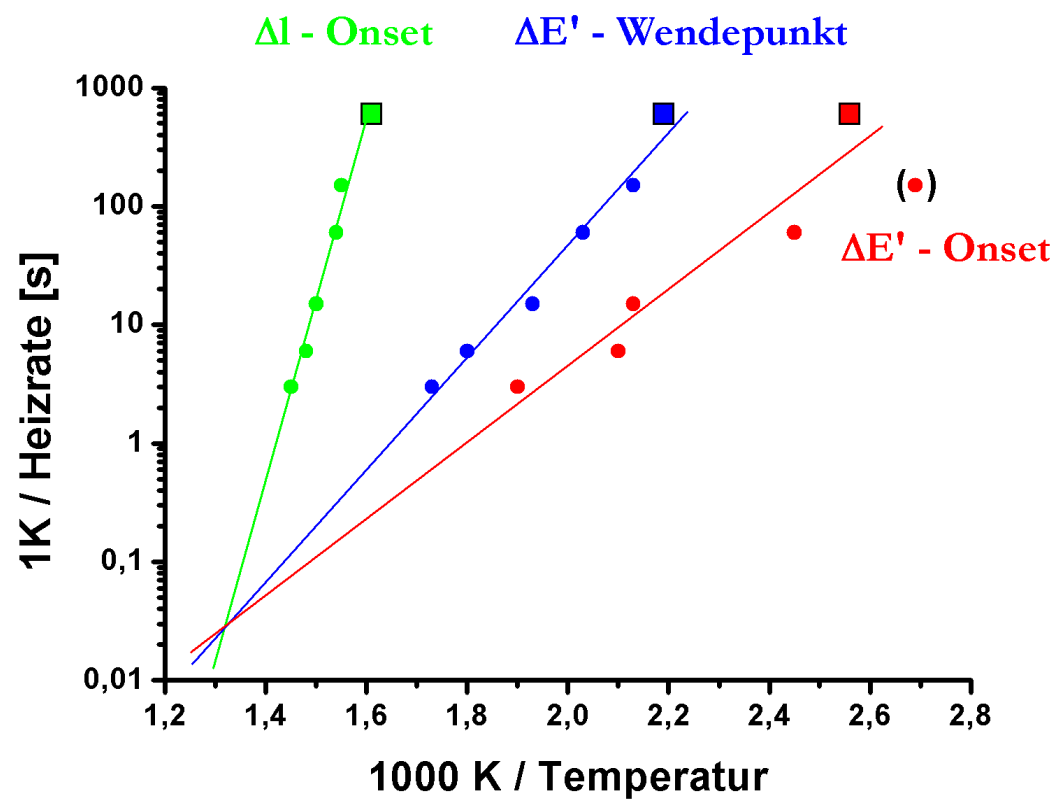

Abbildung 4.4: Einordnung der Relaxationen der langsamen Heizrate (Quadrate mit schwarzem Rand) in eine Kissingerauftragung zum Mergingverhalten nach Hachenberg. ${ }^{[85]}$ In rot ist der Onset und in blau der Wendepunkt des Speichermoduls, sowie in grün der Onset der Probenlänge aufgetragen. Die runden Datenpunkte wurden von Hachenberg et al. ${ }^{[85]}$ übernommen. Alle Messpunkte wurden mit einem linearen Fit extrapoliert.

Wendepunkt (blau) des Speichermoduls - siehe Kapitel 1.4.2) bestimmt. Hierfür wurde die inverse Heizrate halblogarithmisch gegenüber der inversen Temperatur aufgetragen. Hachenberg et al. konnten dabei deutlich verschiedene Temperaturabhängigkeiten für den $\alpha$ - und $\beta$ - Prozess feststellen, wobei durch lineare Extrapolation der Messdaten eine Merging-Temperatur bestimmt wurde, bei der die beiden Prozesse zusammenlaufen. ${ }^{[85]}$

Um die Relaxationen bei der langsamen Heizrate mit denen in der Literatur diskutierten vergleichen zu können, wurden für weitere Heizraten Werte von Hachenberg et al. ${ }^{[85]}$ übernommen (Kreise). Zu erkennen ist, dass die in dieser Arbeit bestimmten Datenpunkte mit den linearen Regressionen der bisher bestimmten Werte gut übereinstimmen. Dies spricht dafür, dass keine neuen Relaxationsprozesse angeregt werden, sondern die langsame $\beta$ - Relaxation auch bei diesem neuen Dämpfungsmaximum die dominierende Relaxationsart ist. Mit den neuen Werten und der Ausklammerung des Datenpunktes für den 
Onset des Speichermoduls bei einer inversen Heizrate von $150 \mathrm{~s}^{2}$ lässt sich der Mergingpunkt relativ genau auf $755 \mathrm{~K} \pm 10 \mathrm{~K}$ (bisheriger Wert $720 \mathrm{~K} \pm 110 \mathrm{~K}^{[85]}$ ) bestimmen. Zur weiteren Untersuchung des Relaxationsverhaltens unterhalb der Glasübergangstemperatur wurden die Elastizitätsmodule sowohl beim Erwärmen als auch beim Abkühlen bei einer Rate von $1 \mathrm{~K} / \mathrm{min}$ bestimmt. Die verwendeten Heiz- und Kühlrampen sind in Abbildung 3.6 und die bestimmten Module in 3.7 und $3.8 \mathrm{zu}$ sehen. Im Verlustmodul ist im ersten Durchlauf ein Absinken zu erkennen, welches Alterungsprozessen, wie sie z. B. bei Bedorf ${ }^{[27]}$ diskutiert wurden, entspricht (siehe auch Kapitel 1.4.2). Bei den weiteren Durchläufen ist erst eine Veränderung ab ca. $500 \mathrm{~K}$ zu sehen, wobei eine Hysterese zwischen Aufwärmen und Abkühlen zu beobachten ist. Über ein ähnliches Verhalten berichteten Khonik et al., wobei eine wiederkehrende Hysterese in Dämpfungsmessungen von amorphem $\mathrm{Pd}_{40} \mathrm{Cu}_{30} \mathrm{Ni}_{10} \mathrm{P}_{20}$ unterhalb der Glasübergangstemperatur beobachtet wurde. Dieses Verhalten wurde über ein größer werdendes freies Volumen beim Erwärmen erklärt, welches strukturelle Relaxationen hervorruft. ${ }^{[99]}$ Im Vergleich zum Speichermodul bei Messungen dieser Arbeit (siehe Abb. 3.8) ist zu erkennen, dass keine Hysterese auftritt. Ein starkes Zunehmen im Modul kann vor allem beim Abkühlen beobachtet werden. Beim Aufwärmen ist nur beim zweiten Durchlauf eine starke Änderung zu sehen, wobei der Modul zunächst abfällt und danach wieder ansteigt. Beim Erwärmen wird jedoch nie ein so hoher Modul wie beim Abkühlen erreicht. Dieser Befund kann wie bei Khonik et al. über strukturelle Relaxationen, in diesem Fall über die langsame $\beta$ - Relaxation, erklärt werden. Beim Erwärmen werden zunächst Prozesse angeregt, welche zu einer Alterung und somit zu einem zunächst sinkenden Verlustmodul führen. Bei weiteren Durchläufen konkurriert bei einer Erwärmung die Alterung mit dem linearen Erwichen der Matrix durch thermische Ausdehnung des Materials, so dass keine großen Änderungen festzustellen sind. ${ }^{[22,100]}$ Bei einer Abkühlung jedoch steht dem Material genügend Energie zur Verfügung, um in der PEL in tiefere Subbasins zu gelangen, wobei durch fehlende thermische Anregung keine höheren Niveaus erreicht werden können. Allgemein ist dieser Effekt im Speichermodul stärker zu erkennen, da dieser sensibler auf strukturelle Änderungen reagiert als der Verlustmodul. ${ }^{[42]}$ Die zu erkennende Hysterese im Verlustmodul kann ebenfalls hierüber erklärt werden. Es werden thermisch langsame $\beta$ - Relaxationen angeregt, was bedeutet, dass höhere Subbasins

${ }^{2}$ Dieser Datenpunkt konnte bei keiner eigenen Messung reproduziert werden 
erreicht werden müssen. Beim Abkühlen hingegen kann das Material leichter in tiefere Subbasins gelangen, da genügend Energie vorhanden ist, um kleine Barrierenhöhen zu überwinden. Es können schneller tiefere Bereiche in der PEL erreicht werden, als beim Erwärmen höhere angeregt werden können (siehe Abb. 4.5). Bei der Annahme, dass die Barrierenhöhen der Subbasins unsymmetrisch angelegt sind, ${ }^{[101]}$ muss für ein Absinken nur die Energie E1 vorhanden sein, beim Erreichen höherer Subbasins wird hingegen E2 benötigt. Hierdurch lässt sich der steilere Verlauf im Verlustmodul beim Abkühlen verstehen.

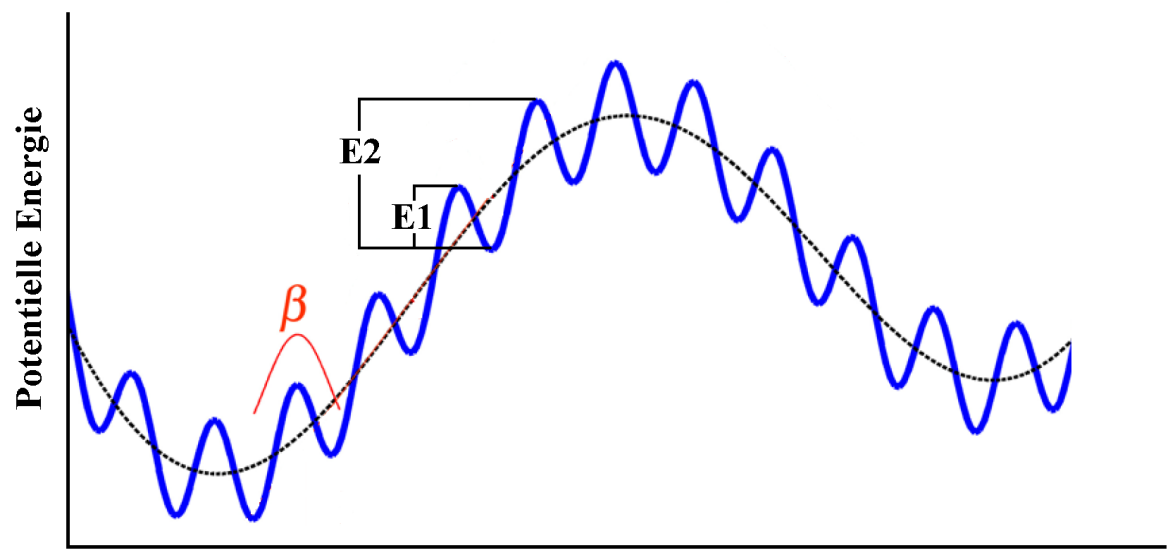

Generalisierte Koordinate

Abbildung 4.5: Abhängigkeit der potentiellen Energie von einer generalisierten Koordinate nach Harmon ${ }^{[50]}$. Die Energie zum Erreichen eines höheren Subbasins innerhalb der PEL (E2) ist größer als die zur Alterung benötigte (E1).

\section{Fazit:}

Durch Untersuchungen bei langsamen Heizraten konnte ein neues Dämpfungsmaximum unterhalb der Glasübergangstemperatur festgestellt werden, welches möglicherweise einem strukturellen Übergang entspricht, wie er in Kolloidsystemen beschrieben wurde. ${ }^{[93-96]}$ Dieser bewirkt durch eine Veränderung der Bindungsverhältnisse eine erleichterte Anregung der langsamen $\beta$ - Relaxation. Ebenfalls konnte die heizratenabhängige Glasübergangstemperatur verfolgt und mit der Kinetik des $\alpha$ - Prozesses in Verbindung gebracht werden, da eine steifere Matrix die kooperativen $\beta$ - Prozesse hemmt und somit zu einer Erhöhung von $\mathrm{T}_{G}$ führt.

Eine starke Alterung der Gläser konnte vor allem beim Abkühlen der Proben beobachtet und mit strukturellen $\beta$ - Relaxationen in Verbindung gebracht werden. Die Barrie- 
renhöhe zwischen den Subbasins ist somit wichtig bei diesen Prozessen und spricht dafür, dass Alterungsprozesse freiwillig ablaufen, da die Barrierenhöhe für Subbasins unsymetrisch angelegt ist und für ein Erreichen tiefer gelegener Subbasins eine geringere Aktivierungsenergie gebraucht wird als bei einer Verjüngung des Materials. 


\subsubsection{Einfluss von Kraftänderungen auf das mechanische Verhalten von amorphem PdCuSi bei Spannungs-Dehnungskurven}

Das Verständnis des Übergangs vom festen, amorphen Zustand in die unterkühlte Schmelze ist zur Erklärung der mechanischen Eigenschaften von Gläsern fundamental. ${ }^{[102]}$ Hierzu werden neben der Erwärmung von Gläsern, wie z. B. bei dynamisch mechanischen Analysen, auch Spannungs-Dehnungskurven untersucht. Der Übergang in die unterkühlte Schmelze wird dabei als der Übergang vom linearen in den nichtlinearen Bereich diskutiert, wobei die unterkühlte Schmelze im Bereich des viskosen Fließens nach dem Yielding Point erreicht ist (siehe Kapitel 1.3.1.2 und 1.4.1). In der Literatur wird für die veränderten mechanischen Eigenschaften der Übergang von der $\beta$ - hin zur $\alpha$ - Relaxation diskutiert (siehe Kapitel 1.4.1). Die Anregung von energetisch höher gelegenen Subbasins bis hin zum Erreichen eines neuen Metabasins im Sinne des Modells der potentiellen Energielandschaft wird hierfür als mögliche Erklärung vorgeschlagen. ${ }^{[50]}$ Zur Untersuchung dieses Modells werden Spannungs-Dehnungskurven bei verschiedenen Kraft- bzw. Dehnungsraten aufgenommen, wobei diese temperaturabhängig Veränderungen aufweisen. ${ }^{[103]}$ Es zeigt sich, dass eine homogene Verformung der untersuchten amorphen Materialien nur bei Temperaturen nahe $\mathrm{T}_{G}$ oder bei sehr geringen Dehnungsraten erreicht werden. ${ }^{[103]}$ Schnelle Kraftänderungen bewirken ein steiferes und härteres Verhalten der Materialien, was in einer Erhöhung des Elastizitätsmoduls bzw. des Yielding Points zu erkennen ist. In Abbildung 3.9 ist genau dieser Fall bei einer Haarprobe zu sehen, da sich das Modul bei einer Erhöhung der Kraftänderung um einen Faktor 10 (von $1 \mathrm{mN} / \mathrm{min}$ auf $10 \mathrm{mN} / \mathrm{min}$ ) um $5 \%$ erhöht und auch der Yielding Point deutlich zu größeren Kräften (122 MPa zu $131 \mathrm{MPa}$, entspricht einer Erhöhung der Kraft um 9 \%) verschoben ist. Bei der Untersuchung an amorphem PdCuSi konnte dieser Befund bestätigt werden (siehe Abb. 3.10). Es ist ein steiferes Verhalten der Probe (Versuchstemperatur etwa $\mathrm{T}_{G}$ ) von $30 \% \mathrm{im}$ Modul zu beobachten, wobei die Kraftänderung nur um den Faktor 2,5 (von 1,0 mN/min auf 2,5 mN/min) erhöht wurde.

Dieses Ergebnis entspricht dem aus Kapitel 4.1.1, wobei die Änderung der Heizrate ebenfalls eine Verschiebung des Überganges $\left(\mathrm{T}_{G}\right)$ zu höheren Temperaturen mit größerer Heizrate bewirkt. Lu et al. berichten auch von einer Verschiebung des Yielding Points zu höheren Spannungen und Dehnungen bei einer größeren Dehnungsrate. ${ }^{[103]}$ Bei diesen 
Versuchen ist wie in Abbildung 3.10 auch eine Erhöhung des Elastizitätsmoduls bei größer werdender Dehnungsrate zu erkennen. Dieser Effekt wird ebenfalls als identisch zur Erniedrigung der Temperatur beschrieben. ${ }^{[103]}$

In Abbildung 3.11 sind bei gleicher Spannungsänderung Spannungs-Dehnungskurven bei verschiedenen Temperaturen zu sehen. Dabei fällt auf, dass das Material bei höheren Temperaturen früher in den Bereich der unterkühlten Schmelze übergeht bzw. bei kleineren Temperaturen ein höheres Elastizitätsmodul bestimmt wird (siehe Abb. 3.12).

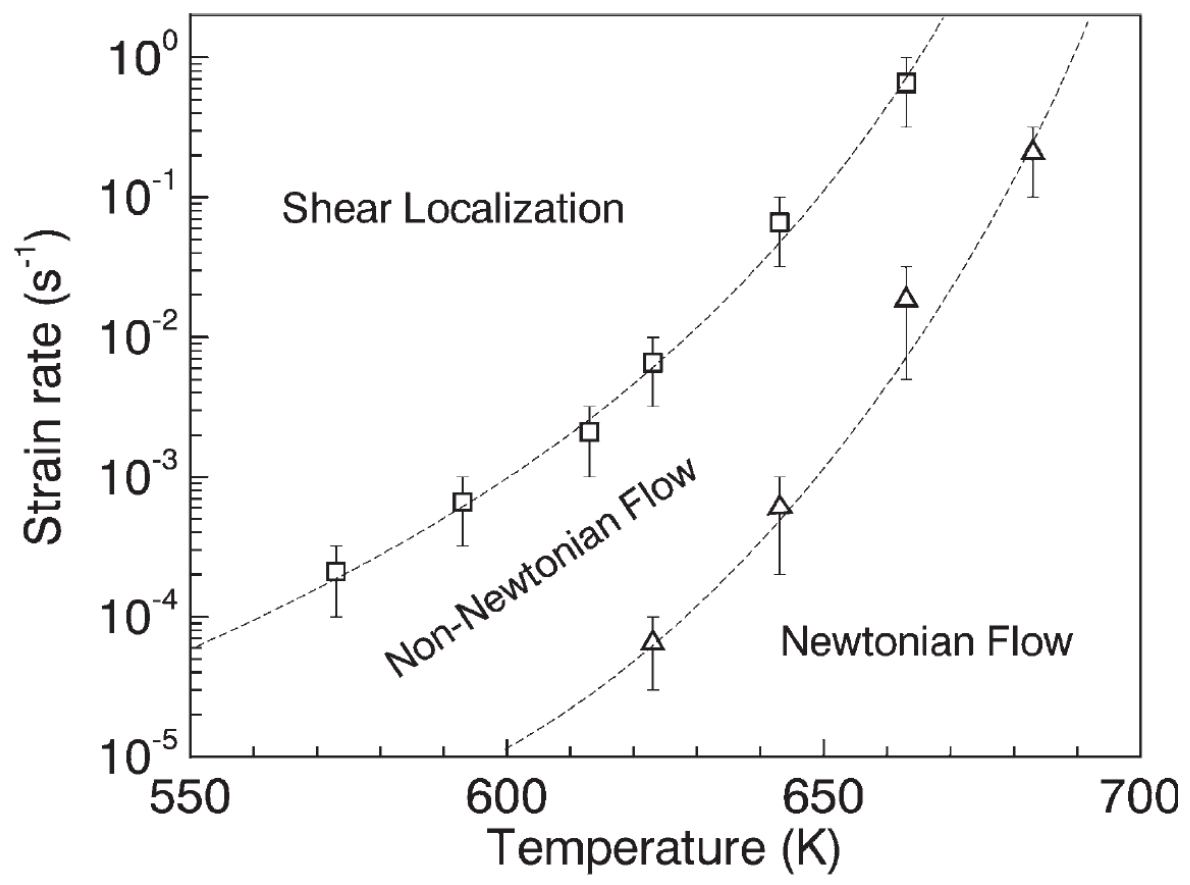

Abbildung 4.6: Abhängigkeit des Überganges von homogener zu nichthomogener Deformation und vom Newtonischen zum nicht-Newtonischen Fließen über die Änderung der Dehnungsrate bzw. der Temperatur von amorphem $\mathrm{Zr}_{41,2} \mathrm{Ti}_{13,8} \mathrm{Cu}_{12,5} \mathrm{Ni}_{10,0} \mathrm{Be}_{22,5} \cdot{ }^{[103]}$

Eine mögliche Erklärung ist, dass über die Temperatur energetisch höher gelegene Subbasins erreicht werden, so dass eine kleinere Änderung in der Spannung ausreicht, die Barrierenhöhe so weit zu erniedrigen, dass ein Übergang erfolgen kann. Die Verschiebung durch eine Änderung in der Spannungsrate scheint einen ähnlichen Effekt zu erzeugen. Dabei scheinen schnelle Änderungen dem Übergang entgegenzuwirken. Dies könnte auf die Verschiebung der Prozesse mit der geänderten Beobachtungszeit durch die schnellere Anregungsfrequenz zurückzuführen sein (siehe Kapitel 1.4). So könnte eine schnelle Änderung der Spannung bzw. der Dehnung zur Folge haben, dass der lineare, elastische 
Bereich nur bei höheren Temperaturen verlassen werden kann. Lu et al. erklären dies über eine Verschiebung des Materialverhaltens mit der Dehnungsrate bzw. der Temperatur zwischen drei Bereichen. Bei schnellen Änderungen bzw. kleinen Temperaturen sind Scherprozesse für Deformationen verantwortlich. Bei einer Reduzierung der Dehnungsrate oder einer Erhöhung der Temperatur zeigt das Material zunächst ein nicht-Newtonisches und nach weiteren Reduzierungen ein Newtonisches Fließverhalten (siehe Abb. 4.6). ${ }^{[103]}$ Der Übergang vom linearen zum nichtlinearen Bereich mit Änderung der Temperatur bei großen Spannungsänderungen von $300 \mathrm{mN} / \mathrm{min}$ ist in Abbildung 3.13 dargestellt. Hierbei wurde unterschieden, ob die Kraft erhöht (Bildteil a) oder erniedrigt wird (Bildteil b). Bei Temperaturen weit unterhalb der Glasübergangstemperatur kann nur rein elastisches Verhalten beobachtet werden, wobei die Kraft nicht für eine inhomogene Verformung reicht. Bei Temperaturen nahe $\mathrm{T}_{G}$ ist jedoch sowohl der Übergang in den nichtlinearen Bereich als auch eine bleibende Deformation nach Zurückfahren der Kraft (Bildteil b) gut zu erkennen. Bei hohen Temperaturen ist jedoch eine Änderung vom inelastischen zum elastischen Verhalten zu beobachten, indem sich das Material bei kleiner werdender Kraft wieder zusammenzieht. Dies ist als Änderung in den Bereich zu bewerten, in dem die angelegte Spannung nicht mehr ausreicht, um die Barrierenhöhe der PEL weit genug zu erniedrigen, um einen $\alpha$ - Prozess zu ermöglichen. In den im linearen Bereich bestimmten Elastizitätsmodulen (siehe Abb. 3.14) ist ein mit größer werdender Temperatur starkes Absinken zu erkennen, welches als temperaturabhängiger Übergang in die unterkühlte Schmelze zu bewerten ist. Hierbei sind zwei Bereiche in den Modulen zu sehen. Im Temperaturbereich von $300 \mathrm{~K}$ bis $600 \mathrm{~K}$ ist für die Krafterniedrigung ein größerer Modul als bei den Messungen der Krafterhöhung zu erkennen. Dies ist über eine Alterung des Materials durch das Auslagern bei der jeweiligen Versuchstemperatur (jeweils insgesamt ca. 15 min.) während des Versuches erklärbar. ${ }^{[61]}$. In der Nähe zu $\mathrm{T}_{G}$, bei Temperaturen über $600 \mathrm{~K}$, sind jedoch die Module für beide Kraftänderungen gleich, da das Material stark plastisch verformt wird und somit keine „Erinnerung“ an den vorherigen Zustand mehr besitzt.

Zur genaueren Untersuchung des linearen, elastischen Verhaltens wurde mit sehr langsamer Dehnungsrate $\left(1 \cdot 10^{-7} \mathrm{~s}^{-1}\right)$ bei Raumtemperatur eine Spannungs-Dehnungskurve aufgenommen (siehe Abb. 3.15). Es wurde dabei jedoch nur eine sehr geringe Dehnung von unter einem Prozent bis zum Riss der Probe erreicht. Bei der Betrachtung eines Aus- 
schnittes der Messung (Abb. 3.16) ist eine periodische Änderung der benötigten Kraft, um die Dehnungsrate zu erzeugen, zu erkennen. Außerdem wird die Kraft innerhalb der Periode unregelmäßig reguliert. Neudecker et al. berichten von lokalisierten Scherprozessen, welchen lokale Yielding Points mit Clustern von etwa 10 Atomen zugeordnet werden können. ${ }^{[46]}$ Diese basieren auf lokalen mechanischen Spannungen und bewirken größere STZ Bereiche mit etwa 250 Atomen bis hin zu einem globalen Yielding Point mit vernetzten STZ-Clustern bis zu 1200 Atomen. Dies zeigt, dass auch unterhalb der Glasübergangstemperatur zur Reduzierung der inneren Spannung sowohl lokal als auch global ein Überschreiten von Barrieren der PEL möglich ist.

Da bei den in dieser Arbeit gezeigten Messungen mit sehr kleiner Dehnungsrate (siehe Abb. 3.15 und 3.16) jedoch über das gesamte Material gemittelt wird, ist es unwahrscheinlich, einzelne STZs erkennen zu können. Diese könnten jedoch Scherbänder erzeugen, welche zu einem Rutschen der Probe führen, aber nicht durch das gesamte Material reichen. Erst bei einem Durchlaufen eines Scherbandes reißt die Probe, was bei diesen Experimenten auch bei sehr kleinen Dehnungen zu beobachten war. Solche Relaxationen im elastischen Bereich wurden ebenfalls bei Drucktests beschrieben. Dalla Torre et al. zeigten, dass sich durch das Eindrücken eines Nano-Indenters Scherbänder bilden, welche nicht zu einem Bruch, aber zu einem Rutschen in der Probe führen. ${ }^{[107]}$ Außerdem berichten David et al. davon, dass bei molekular dynamischen Simulationen quadrupolförmige STZ Bereiche beobachtet wurden, welche eine Vielzahl von Barrieren mit niedriger Energie erzeugen, wobei auch bei kleinen Spannungen Prozesse, wie oben beschrieben, vorhanden sind. ${ }^{[104]}$ Über Röntgenanalysen konnten auch solche unelastischen Veränderungen in der Konfiguration der nächsten Nachbaratome nachgewiesen werden. ${ }^{[106]}$ Unter der Annahme, dass beim Glasübergang eine Wechselbeziehung von lokalen, mechanisch unstabilen Bereichen vorherrscht, können unter Spannungseinfluss einige dieser Bereiche auch vor der Glasübergangstemperatur plastische Eigenschaften aufweisen. ${ }^{[108,109]}$ Somit wäre es möglich, dass die gemessenen schnellen Veränderungen in der Kraft, die nötig sind, um die eingestellte Dehnungsrate zu erzeugen, ebenfalls durch lokale plastische Veränderungen erzeugt werden. Die Messung bei sehr langsamen Dehnungsraten könnte somit ein weiteres Indiz dafür sein, dass auch im linearen Bereich einer Spannungs-Dehnungskurve irreversible Veränderungen auftreten. 


\section{Fazit:}

Bei der Untersuchung von Spannungs-Dehnungskurven konnte eine sehr ähnliche Auswirkung von Temperatur und Spannung auf das mechanische Verhalten von metallischen Gläsern beobachtet werden. Dabei bewirkte eine Reduzierung der Spannungsänderung eine Verschiebung des Überganges vom elastischen zum plastischen Bereich zu tieferen Spannungen. Dieser Effekt wurde ebenfalls bei einer Erhöhung der Temperatur festgestellt. Als Erklärung könnte eine Veränderung des Fließverhaltens von nicht-Newtonischen zum Newtonischen über die Verschiebung der Zeitskala dienen. Außerdem wurde der Übergang zum nichtlinearen Bereich systematisch mit einer Erhöhung der Temperatur untersucht, so dass die Veränderung im elastischen Verhalten sowohl auf eine thermische Anregung von energetisch höher gelegenen Subbasins als auch auf eine Erniedrigung der Barrierenhöhe innerhalb der PEL zurückzuführen ist. Bei sehr langsamen Dehnungsraten konnte auch innerhalb des linearen Bereiches der Spannungs-Dehnungskurve ein Indiz für plastische Prozesse gefunden werden, welche durch lokale irreversible Veränderungen erzeugt werden könnten. 


\subsubsection{Spannungsabhängiges Dämpfungsverhalten von PdCuSi}

Das Relaxationsverhalten von amorphen Festkörpern unterhalb der Glasübergangstemperatur wird in der Literatur häufig mit kettenartigen, kooperativen Bewegungen diskutiert (langsame $\beta$ - Relaxation). ${ }^{[43]}$ Diese Relaxationen stellen möglicherweise die Ursache zur Entstehung von Scher-Transformations-Zonen dar und sind der dominierende Prozess bis hin zu $\mathrm{T}_{G} \cdot{ }^{[47]}$ Innerhalb des in Kapitel 1.4.1 vorgestellten Bildes einer potentiellen Energielandschaft werden diese Relaxationen übersetzt in reversible Anregungen energetisch verschiedener Subbasins, welche innerhalb eines Metabasins angelegt sind. ${ }^{[47]}$ Johnson und Samwer haben hierzu vorgeschlagen, dass eine äußere Spannung die Barrierenhöhe zwischen zwei Metabasins reduzieren kann, was somit zu einem früheren Übergang in den $\alpha$ - Prozess führt. In diesem Kapitel werden die vorgestellten Ergebnisse (siehe Kapitel 3.1.3) der Creep/Recoverykurven hinsichtlich dieser Theorie diskutiert. Dazu wird die Dämpfung, bestimmt über die Delay-Zeiten unterhalb von $\mathrm{T}_{G}$, bei verschiedenen, konstanten Temperaturen miteinander verglichen. Damit sichergestellt werden kann, dass nur die schnellen Relaxationsprozesse untersucht werden, ist hierfür nur das anfängliche Kriechen von Interesse (siehe Kapitel 1.3.1.3).

Der Vergleich einer kristallinen und einer amorphen PdCuSi-Probe (siehe Abb. 3.17) zeigt, dass der Kristall im Gegensatz zum Glas ein rein elastisches Antwortverhalten auf die angelegten Kräfte zeigt. Es sind nur gerätespezifische Dämpfungen zu erkennen, weshalb anschließend eine auf den Kristall normierte Delay-time verwendet wurde. Außerdem sind die Delay-Zeiten des Kristalls über den gesamten untersuchten Temperaturbereich konstant (siehe Abb. 3.18). Das Glas hingegen zeigt bei höheren Temperaturen ein viskoelastisches Verhalten, was sich in einer größeren Dämpfung und somit auch einer größeren Delay-time bemerkbar macht.

Eine Abhängigkeit zur äußeren Spannung wird in Abbildung 3.18 sichtbar. Dabei ist eine Aufspaltung in der Delay-time ab $425 \mathrm{~K}$ zu erkennen, wobei die Dämpfung bei der höheren Spannung größer ist. Bei den bestimmten Elastizitätskonstanten (siehe Abb. 3.19) lässt sich auch ein erstes Indiz dafür finden, dass die Glasübergangstemperatur durch eine äußere Spannung verschoben wird. Dies wird im Kapitel 4.1.4 ausführlich diskutiert.

Die Spannungsabhängigkeit der Dämpfung scheint darauf hinzuweisen, dass sich die Position in der PEL durch die angelegte Kraft variieren lässt. In Abbildung 3.20 lässt 
sich erkennen, dass die Antwort auf die Kraftsprünge unabhängig von der eingestellten Sprunghöhe ist. Eine mögliche Erklärung hierfür bietet die Annahme, dass die Barriere bei Erhöhung der Kraft immer weiter erniedrigt wird. Dies spiegelt sich in der größer werdenden Delay-time wieder, da die Dämpfung zunimmt. Bei jedem Sprung zurück auf die konstante, untere Kraft wird wieder dieselbe Position innerhalb der PEL erreicht. Somit ist die Delay-time bei einer Spannung und einer Temperatur konstant und eine Messgröße für die Dämpfung des Systems. Eine Begründung hierfür kann sein, dass die energetische Position innerhalb der PEL unverändert bleibt, es findet also keine zusätzliche Anregung von Relaxationen statt, sondern die Krümmung der PEL verändert sich. Dies bedeutet, dass die Probe eine weichere Matrix hat und somit die Dämpfung zunimmt. Dabei sind die Ergebnisse unabhängig von der Sprungrichtung der Creep/Recoverymessung, was aus Abbildung 3.21 erkennbar ist.

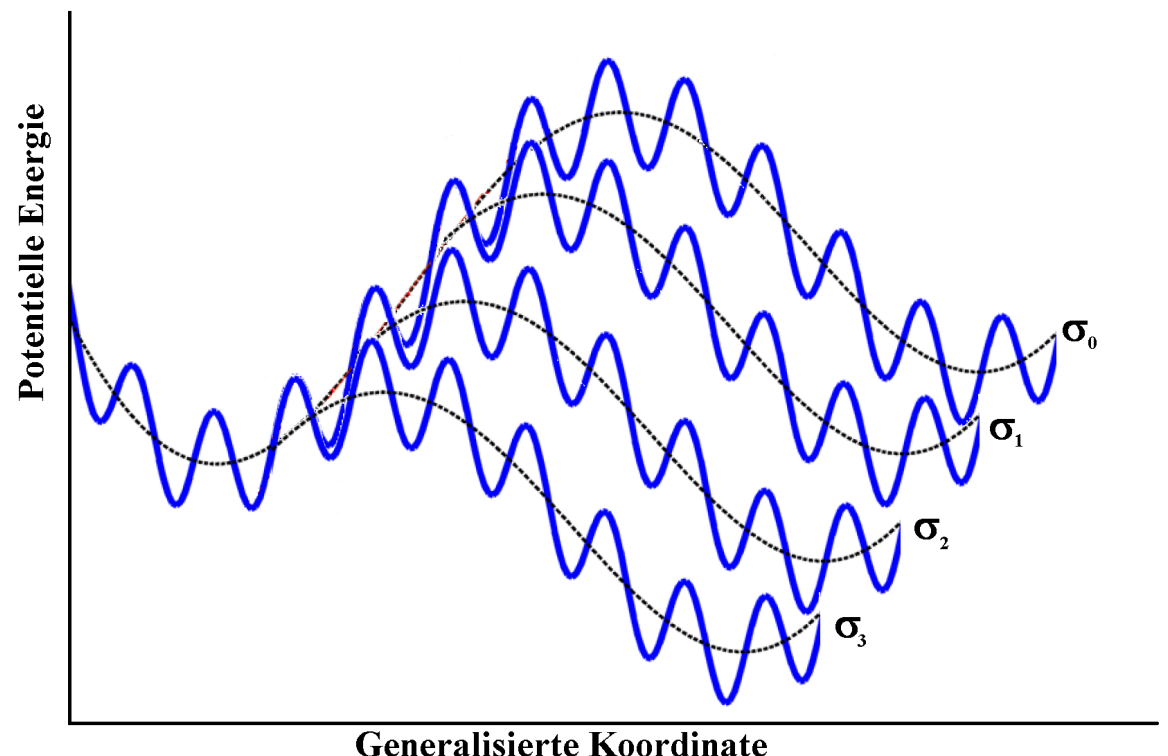

Abbildung 4.7: Schematische Abnahme der Barrierenhöhe der PEL durch eine äußere Spannung $\sigma_{0}<\sigma_{1}<\sigma_{2}<\sigma_{3}$

Die Einstellung der Position innerhalb der PEL ist schematisch in Abbildung 4.7 dargestellt, wobei der Einfluss auf die Sub-Struktur nicht betrachtet wird. Zu sehen ist, dass bei einer größeren, äußeren Spannung $\left(\sigma_{0}<\sigma_{1}<\sigma_{2}<\sigma_{3}\right)$ die Barrierenhöhe abnimmt. Dies hat zur Folge, dass sich die Krümmung der PEL ändert. Ebenfalls ist zu erkennen, weshalb erst bei Temperaturen nahe $\mathrm{T}_{G}$ eine Veränderung in den Delay-Zeiten zu beobachten ist. Dadurch, dass gerätebedingt nur Kräfte bis maximal $1 \mathrm{~N}$ verwendet 
werden konnten, muss der Zustand innerhalb der PEL soweit angeregt werden, bis eine energetisch höhere Position erreicht wird. Erst dann sind spannungsabhängige Änderungen in der Delay-time zu erkennen. Bei tieferen Temperaturen befindet sich die Probe in einem tieferen Subbasin mit steilerer Krümmung des Metabasins, so dass eine kleine Veränderung nicht ausreicht, um diese mit der Auflösung der DMA 7 bestimmen zu können.

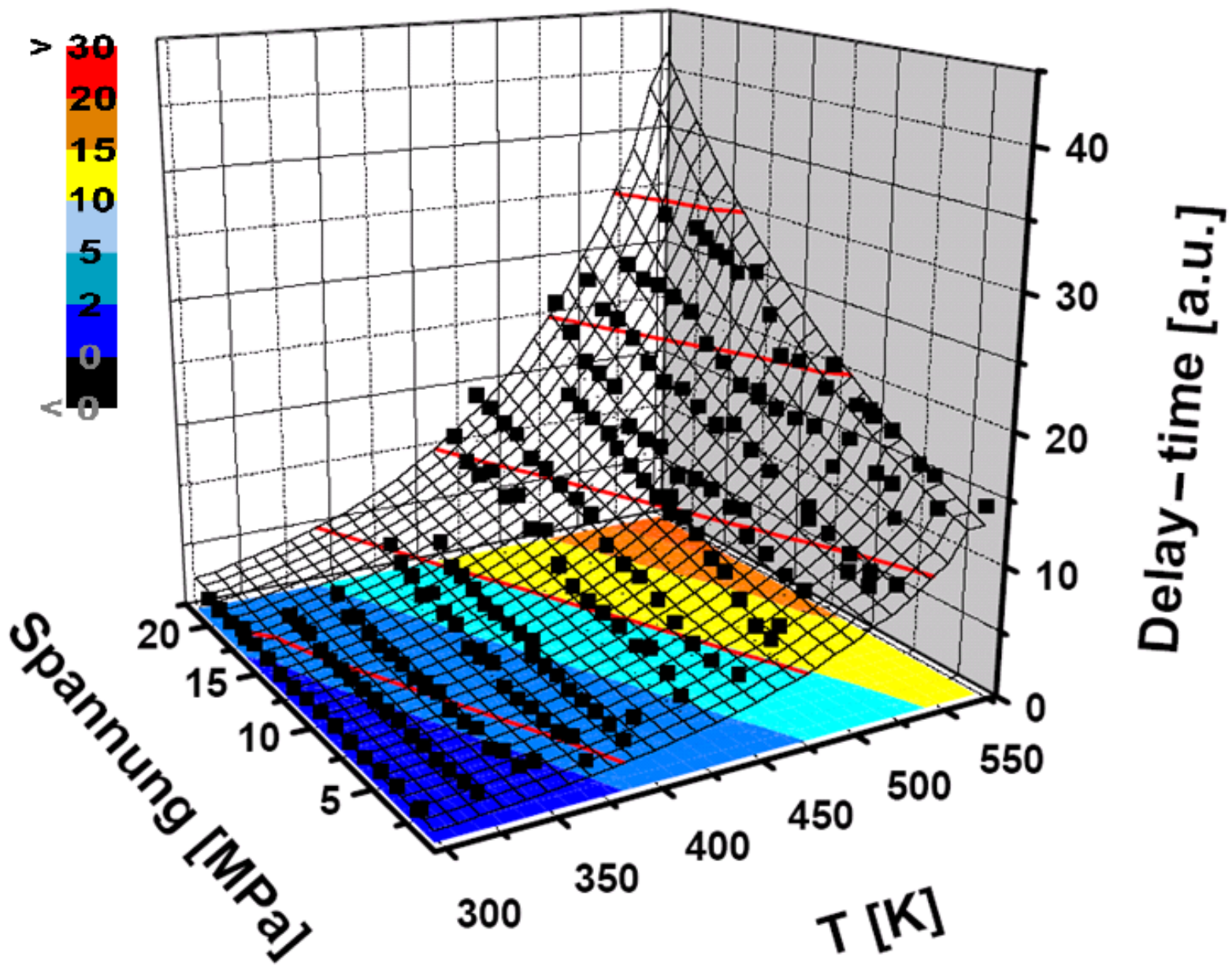

Abbildung 4.8: Exponentielle Anpassung der Dämpfungslandschaft nach Messung aus Abbildung 3.22

Der Zusammenhang des Dämpfungsverhaltens zwischen Temperatur und Spannung ist in Abbildung 3.22 dargestellt, wobei eine „Dämpfungslandschaft" aufgestellt wurde. $\mathrm{Zu}$ erkennen ist, dass die Dämpfung sowohl mit steigender Temperatur als auch mit höherer Spannung größer wird. Hierbei nimmt jedoch das Dämpfungsverhalten nicht linear zu, wie es in den vorherigen Messungen erschien, sondern es liegt eine Krümmung vor. Sowohl mit steigender Temperatur als auch mit steigender Spannung kann die 
Dämpfungszunahme näherungsweise exponentiell beschrieben werden. Dieses Verhalten ist in Abbildung 4.8 angepasst.

Die Messpunkte sind in schwarz zu erkennen und mit einem exponentiell dargestellten Gitternetz verbunden. Hierfür wurde die Gleichung:

$$
\text { Delay -time }(\text { normiert })=e^{c_{T}+\lambda_{T}\left(T-T_{0}\right)} \cdot e^{c_{\sigma}+\lambda_{\sigma}\left(\sigma-\sigma_{0}\right)}=A \cdot e^{\lambda_{T}\left(T-T_{0}\right)+\lambda_{\sigma}\left(\sigma-\sigma_{0}\right)}
$$

verwendet, da sie sich empirisch als am besten geeignet erwies. Die Parameter sind in Tabelle 4.1 aufgeführt. Dabei ist wichtig zu erwähnen, dass die Delay-time spannungsund temperaturabhängig sowohl multiplikativ miteinander verknüpft zu sein scheint als auch über dieselbe Abhängigkeit zu beschreiben ist. Dies deutet darauf hin, dass es sich um sehr ähnliche Prozesse handeln muss, die angeregt werden, um eine weichere Matrix zu erlangen.

\begin{tabular}{|c|c|}
\hline $\mathrm{A}$ & 4,874 \\
\hline $\mathrm{c}_{T}$ & 0,792 \\
\hline $\mathrm{c}_{\sigma}$ & 0,792 \\
\hline$\lambda_{T}$ & $0,01 \mathrm{~K}^{-1}$ \\
\hline$\lambda_{\sigma}$ & $0,0562 \mathrm{MPa}^{-1}$ \\
\hline $\mathrm{T}_{0}$ & $423,5 \mathrm{~K}$ \\
\hline$\sigma_{0}$ & $10,28 \mathrm{MPa}$ \\
\hline
\end{tabular}

Tabelle 4.1: Verwendete Parameter zur Berechnung des Dämpfungsverhaltens nach Gleichung 4.1.

Der verwendete Parameter $\lambda_{T}$ gibt dabei die Änderung der Dämpfung mit der Temperatur und $\lambda_{\sigma}$ mit der Spannung wieder. Dabei ist zu erwähnen, dass $\lambda_{T}$ mit $0,01 \mathrm{~K}^{-1}$ in derselben Größenordnung liegt, wie die Veränderung des Schermoduls bei Temperaturerhöhung, welche T. Koeppe bei Ultraschallexperimenten bestimmen konnte (0,013 GPa pro K). ${ }^{[22]}$ Eine erkennbare Veränderung der Dämpfung und somit das Einsetzen des $\beta$ - Prozesses ist jedoch bei Raumtemperatur erst ab einer Spannung von 10,28 MPa bzw. bei 423,5 K (bei einer äußeren Spannung von $1 \mathrm{MPa}$ ) zu beobachten. Die Konstanten A bzw. $\mathrm{c}_{T}$ und $\mathrm{c}_{\sigma}$ sind zur Normierung notwendig. 
Die exponentielle Anpassung zeigt jedoch einen gemittelten Fehler von $20 \%$ zu den Messwerten. Hierbei muss aber vor allem darauf verwiesen werden, dass die Streuung der Datenpunkte bei größeren Temperaturen nahe $\mathrm{T}_{G}$ stark zunimmt und somit ebenfalls die Abweichung zum berechneten Gitter.

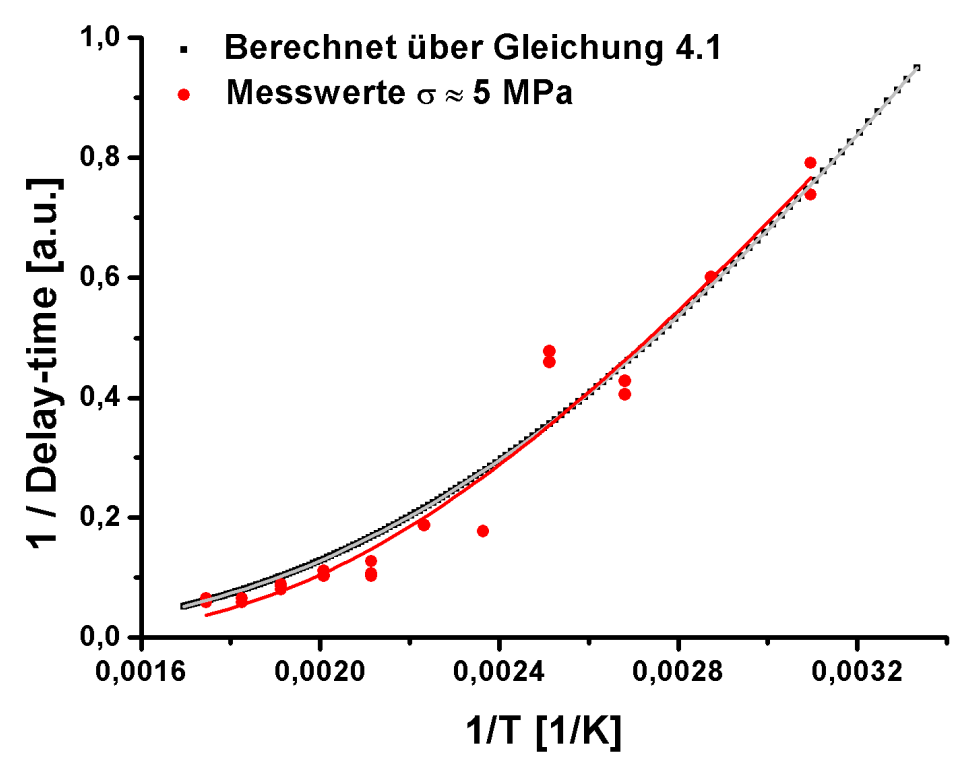

Abbildung 4.9: Vergleich der berechneten inversen Delay-time (schwarze Punkte) gegenüber den Messwerten (rote Punkte) aufgetragen gegen die inverse Temperatur. Die graue bzw. rote Kurve entspricht einem Vogel-FulcherTammann-Verhalten.

Ein Vergleich zwischen den Messpunkten und der berechneten exponentiellen Anpassung der Gleichung 4.1 ist in Abbildung 4.9 zu sehen. Es ist die inverse Delay-time (proportional zur Viskosität) gegenüber der inversen Temperatur bei einer äußeren Spannung von $5 \mathrm{MPa}$ aufgetragen. Die Messpunkte sind in rot und die berechneten in schwarz dargestellt. Es ist zu erkennen, dass die Anpassung gut zu den Datenpunkten passt. Die Streuung ist darüber zu erklären, dass bei der Messung die Spannung zwischen 4,3 MPa und 6,3 MPa schwankt und nicht exakt $5 \mathrm{MPa}$ entspricht. Außerdem tragen die oben genannten Ungenauigkeiten zu den Schwankungen bei. Sowohl für die Messpunkte als auch für die berechneten Datenpunkte wurde ein Vogel-Fulcher-Tammann-Verhalten (VFT) über die Gleichung 


$$
y=y_{0} \cdot e^{\left(\frac{B}{x-H}\right)}
$$

angepasst (in grau bzw. rot dargestellte Kurven). Dabei ist y die inverse Delay-time, welche proportional zur Viskosität ist und x die inverse Temperatur. Es ist zu erkennen, dass die Anpassung sehr gut zu dem Verhalten der Delay-time gegenüber der Temperatur passt. Die verwendeten Parameter sind in Tabelle $4.2 \mathrm{zu}$ sehen.

\begin{tabular}{|c|c|c|c|}
\hline Datenpunkte & $\mathrm{y}_{0}$ & $\mathrm{~B}[1 / \mathrm{K}]$ & $\mathrm{H} \cdot 10^{-6}[1 / \mathrm{K}]$ \\
\hline Messung & 9,28 & $-0,006$ & 630 \\
\hline Berechnet & 19,08 & $-0,01$ & 0,00156 \\
\hline
\end{tabular}

Tabelle 4.2: Verwendete Parameter zur Gleichung 4.2.

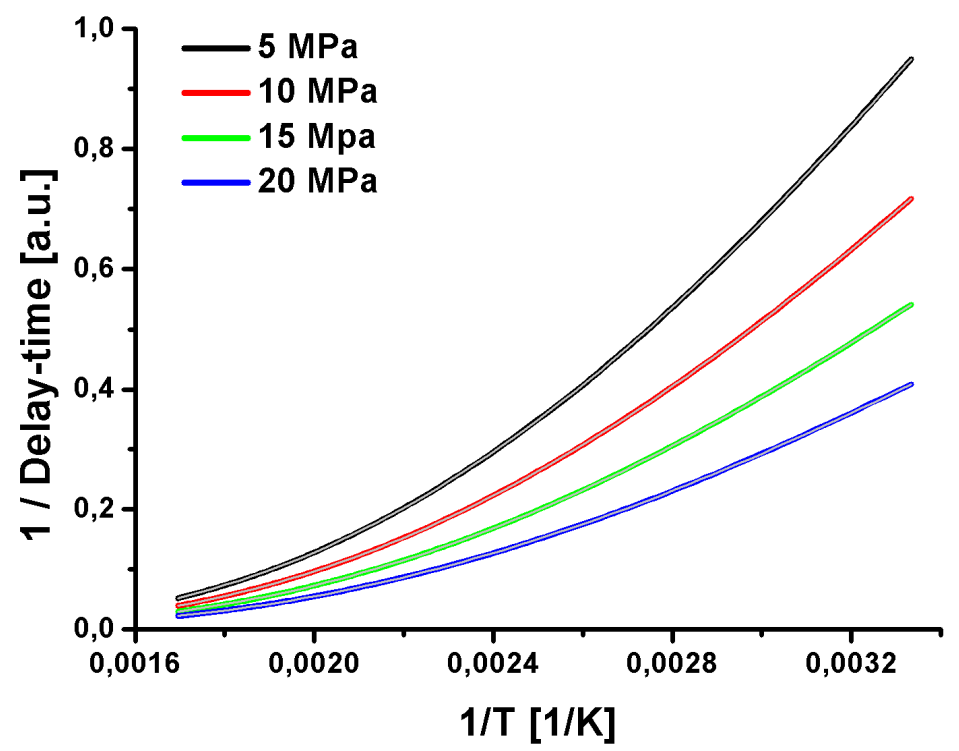

Abbildung 4.10: Vergleich verschiedener Spannungen zur Nachprüfung des Vogel-FulcherTammann-Verhaltens der Delay-time. Die Kurven wurden mit der Gleichung 4.1 berechnet, in grau ist das VFT-Verhalten vergleichend eingezeichnet.

Dass dieses Verhalten auch über den gesamten untersuchten Spannungsbereich gilt, zeigt Abbildung 4.10. Dabei wurden die Spannungen $5 \mathrm{MPa}$ (schwarz), $10 \mathrm{MPa}$ (rot), $15 \mathrm{MPa}$ (grün) und $20 \mathrm{MPa}$ (blau) verglichen und mit einem VFT-Verhalten dargestellt 
(graue Kurven). Die Kurven wurden mit der Gleichung 4.1 und das VFT-Verhalten über Gleichung 4.2 berechnet. Die Parameter sind in Tabelle 4.3 zu sehen.

\begin{tabular}{|c|c|c|c|}
\hline Spannung $[\mathrm{MPa}]$ & $\mathrm{y}_{0}$ & $\mathrm{~B}[1 / \mathrm{K}]$ & $\mathrm{H} \cdot 10^{-9}[1 / \mathrm{K}]$ \\
\hline 5 & 19.08 & $-0,01$ & 1.56 \\
\hline 10 & 14,41 & $-0,01$ & 1.57 \\
\hline 15 & 10.88 & -0.01 & 1.57 \\
\hline 20 & 8.21 & $-0,01$ & 1.56 \\
\hline
\end{tabular}

Tabelle 4.3: Verwendete Parameter zur Gleichung 4.2 für Abbildung 4.10.

Im Vergleich hierzu ist in Abbildung 4.11 das Verhalten der inversen Delay-time gegenüber der Spannung bei konstanter Temperatur aufgetragen. Dabei ist zu beobachten, dass die Dämpfung mit der Spannung exponentiell zunimmt, aber nicht über ein VFT-Verhalten zu beschreiben ist. Da aber mit sehr kleinen Kräften von unter 1 N gearbeitet wurde, scheint die Dämpfung in der Messung (siehe Abb. 3.22) fast linear anzusteigen.

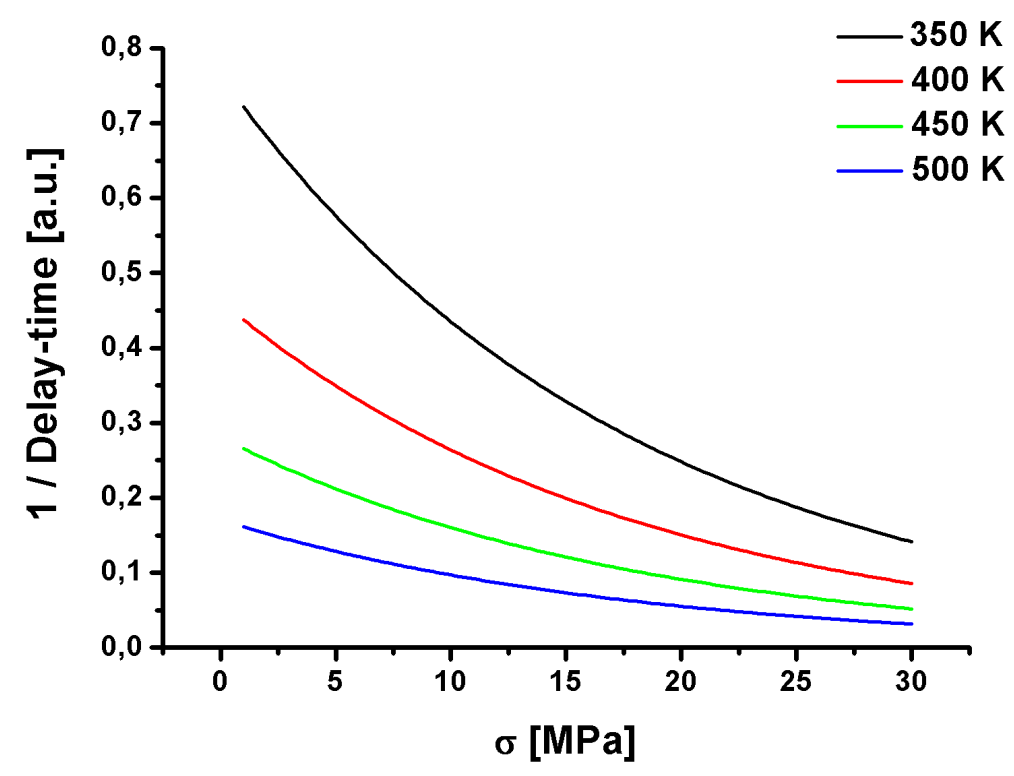

Abbildung 4.11: Vergleich der inversen Delay-time gegenüber der äußeren Spannung bei verschiedenen Temperaturen. Die Kurven wurden über die Gleichung 4.1 berechnet.

Bei der Betrachtung des gesamten Bereiches jedoch (siehe Abb. 4.8) erscheint eine exponentielle Anpassung besser geeignet. Ein sehr ähnliches Verhalten konnten Guan et al. 
über MD-Simulationen an amorphem $\mathrm{Zr}_{50} \mathrm{Cu}_{40} \mathrm{Al}_{10}$ nachweisen. Dabei wird gezeigt, dass die inverse Viskosität bei hohen Temperaturen auch über ein Vogel-Fulcher-TammannVerhalten zu beschreiben ist, jedoch zu niedrigeren Temperaturen in der Nähe von $\mathrm{T}_{G}$ in ein Power-law übergeht. ${ }^{[105]}$

Diese Anpassung der Gleichung 4.1 zeigt, dass erst ab einer Temperatur von über $420 \mathrm{~K}$ (bei einer äußeren Spannung von $1 \mathrm{MPa}$ ) bzw. einer äußeren Spannung von $10 \mathrm{MPa}$ (bei Raumtemperatur) eine signifikante Änderung in der Dämpfung zu erkennen ist (siehe Tabelle 4.1). Dabei liegt diese im selben Temperaturbereich wie bei dynamischen Messungen (siehe Kapitel 3.1.1) der Anstieg zum Wing. Dieser verschiebt sich bei den Creep/Recoverymessungen jedoch spannungsabhängig zu niedrigeren Temperaturen, was den Einfluss der Spannung auf die langsame $\beta$ - Relaxation direkt zeigt.

Die so erhaltene Landschaft wird im Sinne des Bildes der potentiellen Energielandschaft nach der Idee von Johnson und Samwer ${ }^{[47]}$ interpretiert und gibt somit einen Einblick in die Abhängigkeiten der Energielandschaft zu Temperatur und Spannung. Das Material kann entweder über eine angelegte äußere Spannung, durch Verkippen der PEL oder über thermische Anregung in dieselbe Position innerhalb der PEL mit geringerer Steigung gelangen.

Fazit:

Mit Hilfe von Creep/Recoverymessungen konnte gezeigt werden, dass sowohl eine Erhöhung der Temperatur als auch eine äußere Spannung das Dämpfungsverhalten von amorphem PdCuSi unterhalb der Glasübergangstemperatur beeinflusst. Dabei wird das Material weicher, je höher die Temperatur oder die Spannung ist. Ebenfalls wurde eine Verknüpfung der Prozesse empfohlen, da sich ein sehr ähnliches Verhalten in den Dämpfungsänderungen gezeigt hat. Hierbei hat sich eine exponentielle Anpassung als am geeignetsten erwiesen, wobei ein Vogel-Fulcher-Tammann-Verhalten der Delay-time bei konstanter Spannung gefunden wurde. Im Sinne des Bildes der potentiellen Energielandschaft wird dieser Befund mit einer Anregung energetisch höher gelegener Subbasins bei einer Temperaturerhöhung vorgeschlagen, so dass ein energetisch höherer Bereich innerhalb der PEL mit einem Abflachen der Steigung des Metabasins erreicht wird. Der Befund, dass dieser Effekt ebenfalls bei Erhöhung der äußeren Spannung erreicht wird, wird als Indiz dafür bewertet, dass die Energiebarriere kippt und sich somit verringert. Das Material würde ebenfalls in einen Bereich mit flacherer Steigung gelangen können. 


\subsubsection{Glasübergangstemperatur und Aktivierungsvolumen von PdCuSi in Abhängigkeit der äußeren Spannung}

Die erhaltene Spannungs- und Temperaturabhängigkeit des Dämpfungsverhaltens, welche mit einer Beeinflussung der Barrierenhöhe innerhalb der PEL diskutiert wurde (siehe Kapitel 4.1.3), sollte auch Einfluss auf die Glasübergangstemperatur und die dazu notwendigen Relaxationsprozesse nehmen. Hierzu ist es wichtig, sowohl die Elastizitätskonstanten als auch die Glasübergangstemperatur temperatur- und spannungsabhängig hinsichtlich der Einordnung der Ergebnisse in das Bild der PEL zu untersuchen. Dafür müssen auch Relaxationsprozesse, welche nahe und oberhalb der Glasübergangstemperatur ablaufen, genauer bestimmt werden. Dafür werden in der Literatur ScherTransformations-Zonen vorgeschlagen (siehe Kapitel 1.4), die über ein Aktivierungsvolumen studiert werden können. ${ }^{[27,66,67]}$

In diesem Kapitel werden die Ergebnisse (siehe Kapitel 3.1.4) auf die oben genannten Punkte geprüft und hinsichtlich der Einflüsse von Spannung und Temperatur auf die PEL diskutiert. Dazu wurden Creep/Recoverymessungen mit einer konstanten Heizrate aufgenommen. Dabei war nicht wie in den Messungen bei konstanter Temperatur (siehe Kapitel 3.1.3) das Nachkriechen des Materials von Interesse, sondern vor allem der direkte Bereich nach dem Kraftsprung, da hier ein lineares Verhalten zwischen Spannung und Dehnung vorliegt, so dass der Elastizitätsmodul und das Aktivierungsvolumen bestimmt werden konnten (siehe Kapitel 2.1.4).

Dass dieses Verfahren sensibel auf Änderungen im Elastizitätsmodul reagiert, kann in Abbildung 3.24 sehr gut erkannt werden. Es ist der Modul von Raumtemperatur bis hin zum kristallinen Zustand der Probe bestimmt worden. Dabei ist unterhalb der Glasübergangstemperatur (610 K) ein Ansteigen im Modul zu erkennen, welches mit einer Alterung der Probe (siehe Kapitel 1.4.2) in Verbindung gebracht werden kann. Sehr gut ist das folgende, durch das Erweichen der Probe bedingte, Abknicken zu erkennen, welchem sich eine durch ein Minimum gekennzeichnete Kristallisation (640 K) anschließt. Der nachfolgende Anstieg im Modul ist durch das Härten der Probe zu verstehen. Dabei sind weitere Minima zu beobachten, die weitere Kristallisationsstufen kennzeichnen. Zum Abschluss der Messung wird ein Modul erreicht, welcher mehr als das Doppelte des Anfangsmoduls beträgt (85 GPa zu $33 \mathrm{GPa}$ ). Diese Werte passen sehr gut zu den in der 
Literatur bestimmten Elastizitätskonstanten (bei $400 \mathrm{~K} 30 \mathrm{GPa}-47 \mathrm{GPa}^{[14]}, 37 \mathrm{GPa}^{[27]}$ ) und zeigen, dass die Auswertmethode sensibel auf Änderungen im Modul reagiert.

Beim Vergleich von verschiedenen angelegten Spannungen lassen sich die oben beschriebenen Merkmale im Modul ebenfalls erkennen (siehe Abb. 3.25). Dabei fällt jedoch auf, dass der Onset zur Alterung scheinbar keine Korrelation zu den angelegten Kräften aufweist. Ebenfalls lässt sich dieser Punkt nicht mit in das Bild des Mergings (siehe Abb. 4.4) einordnen. Dies kann jedoch daran liegen, dass hier keine dynamische Messung vorgenommen, sondern ein Kraftsprung verwendet wurde und somit auch eine deutlich andere Frequenz zum Abfragen der Prozesse vorlag. Somit können diese Resultate deutlich in der Temperatur von den im Kapitel 3.1.1 vorgestellten Ergebnissen abweichen. Bei allen Messungen kann aber in der Nähe zu $\mathrm{T}_{G}$ eine klare Abhängigkeit des Elastizitätsmoduls zur äußeren Spannung beobachtet werden. Es ist eine Aufspaltung der Module zu erkennen, wobei der Modul der größeren Kraft immer unterhalb der der kleineren liegt. Erst bei beginnender Kristallisation wird dieser Offset wieder kleiner. Dies ist ein eindeutiges Indiz dafür, dass es sich um eine glasspezifische Eigenschaft handelt und dass die Position innerhalb der PEL durch die äußere Kraft verändert und das Material somit weicher wird.

Vor allem jedoch fällt auf, dass sich die Glasübergangstemperatur mit der äußeren Spannung verschiebt. In Abbildung 3.26 ist dieses Ergebnis dargestellt. Eine lineare Abnahme von 1,3 K pro MPa lässt sich bestimmen. Da nur sehr kleine Kräfte von unter einem Newton verwendet wurden, ist es jedoch möglich, dass nur ein sehr kleiner Bereich der Verschiebung betrachtet wurde und somit keine wirkliche Linearität vorliegt. Es ist zu vermuten, dass wie in der Dämpfungslandschaft (siehe Abb. 4.7) eine langsame exponentielle Abnahme möglich ist.

Die Spannungsabhängigkeit könnte sich darüber erklären lassen, dass die Barriere innerhalb der PEL durch die äußere Spannung immer weiter erniedrigt und somit der Übergang zum $\alpha$ - Prozess bei niedrigeren Temperaturen möglich wird. Dabei ist zu beobachten, dass das Material bei den Sprüngen mit der größeren Kraft schon im Bereich der unterkühlten Schmelze vorliegt und beim Sprung zurück auf die kleinere Kraft in einen Bereich vor der Glasübergangstemperatur gewechselt wird. Dadurch, dass die Barriere wieder auf den höheren Wert wechselt, ist es möglich, dass sie bei der kleineren Spannung erst bei höheren Temperaturen überschritten werden kann. 
Dieses Verhalten müsste sich auch im Aktivierungsvolumen bemerkbar machen, da bei der Annahme, dass mehrere $\beta$ - Relaxationen, die mit ihren Spannungsfeldern überlappen, die Auslöser für Scher-Transformations-Zonen sind, das Aktivierungsvolumen bei einer Erniedrigung der Barrierenhöhe kleiner werden sollte. Dies bezieht sich darauf, dass weniger Subbasins erreicht werden müssen, um den Übergang in die $\alpha$ - Relaxation zu gewährleisten.

In Abbildung 3.29 ist das berechnete Aktivierungsvolumen in Abhängigkeit der äußeren Spannung aufgetragen. Zusätzlich ist die Abnahme der zugehörigen Temperatur $\left(\mathrm{T}_{G}\right)$, bei der das Volumen bestimmt wurde, zu sehen. Dabei ist zu erkennen, dass das Aktivierungsvolumen spannungsabhängig kleiner wird. Hierbei ist jedoch eine große Streuung in den Daten zu beobachten, was an der starken Viskositätsänderung liegen kann, die bei $\mathrm{T}_{G}$ vorliegt. Die zur Berechnung verwendeten linearen Bereiche der Creep/Recoverykurven nach dem Kraftsprung sind größer fehlerbehaftet, da diese aufgrund des weicheren Materials kleiner werden und auch eine größere Dämpfung aufweisen. Außerdem ist es möglich, dass durch die starke Viskositätsänderung die bestimmten Werte nicht mehr unabhängig von der Richtung des Kraftsprunges sind. Dies wurde jedoch nicht näher untersucht, da diese Ungenauigkeiten sich nicht auf die Hauptthese der spannungsabhängigen Veränderung des Aktivierungsvolumens auswirken.

Im Vergleich zu MD-Simulationen ist das hier bestimmte Aktivierungsvolumen mit 350 bis 660 Atomen $\left(5,3 \mathrm{~nm}^{3}-9,9 \mathrm{~nm}^{3}\right.$ mit einem Durchmesser von 2,2 nm - 2,7 nm) größer als erwartet. In den Simulationen wird nur ein Volumen bis zu 100 Atomen mit einem Durchmesser von ca. 1,5 nm für STZs beobachtet. ${ }^{[46,71,72]}$ Der Vergleich mit Druckexperimenten von Chen et al., die ein Volumen für STZs von 2,5 $\mathrm{nm}^{3}-6,5 \mathrm{~nm}^{3}$ vermessen haben, ${ }^{66,67]}$ lässt jedoch den Schluss zu, dass in diesen Experimenten dieselben Relaxationsprozesse bestimmt wurden. Außerdem konnten Druckexperimente von Birringer et al. an amorphem $\mathrm{Pd}_{40} \mathrm{Ni}_{40} \mathrm{P}_{20}$ ein Aktivierungsvolumen für STZs von $8,6 \mathrm{~nm}^{3}$ (200 - 600 Atome) diese Größenordnung bestätigen. ${ }^{[110]}$ Es ist jedoch nicht geklärt, ob nur der plastische Bereich der STZs vermessen oder ein Teil des elastischen Spannungsfeldes mit betrachtet wurde.

Auch oberhalb der Glasübergangstemperatur in der unterkühlten Schmelze konnte das Verhalten des Aktivierungsvolumens bestimmt werden. Es konnte eine Abnahme mit steigender Dehnung des Materials beobachtet werden (siehe Abb. 3.32). Dabei kann der 
Verlauf mit einer guten Übereinstimmung mit einem exponentiellen Abfall beschrieben werden. Dies deutet darauf hin, dass in der unterkühlten Schmelze ein HochtemperaturBarrierenlimit für die angeregten STZs im steady-state Fließbereich vorliegt. Dieser konnte mit einem Volumen von ca. 7,49 $\mathrm{nm}^{3}$ (490 Atome) vorhergesagt werden. Dabei muss darauf hingewiesen werden, dass bei den verwendeten Messungen wegen der konstanten Heizrate eine höhere Dehnung auch eine höhere Temperatur bedeutet. Aber die Temperaturzunahme lässt keinen Rückschluss auf eine Abnahme im Aktivierungsvolumen zu. Eine Erklärung für den Verlauf ist, dass durch die größere Dehnung und somit auch der größeren Spannung die Barrierenhöhe erniedrigt wird. Jedoch ist wegen der hohen Temperatur (oberhalb von $\mathrm{T}_{G}$ ) das Material in einem sehr hoch energetischen Bereich innerhalb der PEL, so dass ein Limit für die Barrierenerniedrigung erreicht wird. Da aber nur sehr wenige Messpunkte für diesen Bereich vorliegen, kann hierzu keine endgültige Aussage gemacht werden.

Fazit:

Durch Creep/Recoverymessungen mit einer konstanten Heizrate konnte ein spannungsabhängiges Elastizitätsverhalten von amorphem PdCuSi nahe der Glasübergangstemperatur bestimmt werden. Dabei wurde auch die Glasübergangstemperatur als spannungsabhängig beobachtet, wobei diese mit steigender Spannung abnahm. Als mögliche Begründung wird eine spannungsabhängige Barrierenerniedrigung innerhalb der PEL vorgeschlagen. Durch die äußere Spannung wird ein Übergang in die unterkühlte Schmelze bei niedrigeren Temperaturen möglich. Bei der Annahme, dass überlappende Spannungsfelder der $\beta$ - Relaxation für die Entstehung von Scher-Transformations-Zonen verantwortlich sind, kann über diese Barrierenerniedrigung auch eine Verringerung des Aktivierungsvolumens für STZs erklärt werden. Dadurch, dass eine geringere Aktivierung von $\beta$ - Relaxationen nötig ist, um STZs zu aktivieren, nimmt das Aktivierungsvolumen mit Erhöhung der äußeren Spannung ab. Oberhalb der Glasübergangstemperatur wurde ein Hochtemperatur-Barrierenlimit für STZs vorgeschlagen, da hochangeregte Positionen innerhalb der PEL besetzt sind und somit die Barriere nicht sehr weit erniedrigt werden kann, bis ein $\alpha$ - Übergang angeregt wird. 


\subsubsection{Glasübergangstemperatur und Aktivierungsvolumen von ZrAICu in Abhängigkeit der äußeren Spannung}

In diesem Kapitel werden die Ergebnisse aus dem vorherigen Kapitel 4.1.4 zum spannungsund temperaturabhängigen Elastizitätsverhalten von $\mathrm{PdCuSi}$ mit denen von $\mathrm{ZrAlCu}$ verglichen, damit das Verhalten von fragilen und starken Glasbildnern gegenübergestellt werden kann. Dazu wurden unter identischen Versuchsbedingungen zum PdCuSi Creep/Recoverykurven von $\mathrm{ZrAlCu}$ bei einer konstanten Heizrate aufgenommen. Oxidationseffekte wurden durch eine geschlossene Stickstoffatmosphäre unterbunden. Durch die frühere Kristallisation von $\mathrm{ZrAlCu}$ kann jedoch nur ein kleinerer Bereich in der unterkühlten Schmelze beobachtet werden (siehe Abb. 3.33 und 3.34).

Im Vergleich zum PdCuSi ist ein sehr ähnlicher Verlauf des Elastizitätsmoduls zu bestimmen (siehe Abb. 3.34), wobei unterhalb der Glasübergangstemperatur ein Ansteigen im Modul zu erkennen ist, welches vergleichbar zum PdCuSi mit einer Alterung des Materials erklärbar ist. Der Onset des Anstiegs zeigt dabei aber keine Korrelation zu den verwendeten Kräften. Eine Einordnung in das Mergingverhalten dieses Glasbildners ${ }^{[55}$ ist dabei, analog zum PdCuSi, nicht möglich. Dabei zeigt die Größe des Moduls keine Unterschiede zum Pd-Glas. Zu erkennen ist jedoch, wie in der Literatur ebenfalls beschrieben, ${ }^{[13,14]}$ dass die Glasübergangstemperatur des auf Zirkon basierenden Glases bei gleicher Spannung etwa $15 \mathrm{~K}$ höher als beim Palladium-Glas liegt. Die spannungsabhängige Verschiebung der Glasübergangstemperatur, welche im Kapitel 4.1.4 bei PdCuSi diskutiert wurde, konnte ebenfalls bei $\mathrm{ZrAlCu}$ beobachtet werden (siehe Abb. 3.35). Es ist ein linearer Zusammenhang im untersuchten Spannungsbereich zu beobachten. Dabei ist eine größere Abnahme in der Glasübergangstemperatur (2,35 K pro MPa) als beim Palladium-Glas zu erkennen. Die Erniedrigung der Barrierenhöhe innerhalb der PEL ist auch hier eine sinnvolle Annahme zur Erklärung der Spannungsabhängigkeit. Weshalb die Temperaturabnahme stärker spannungsabhängig zu sein scheint, ist noch nicht geklärt, ein Zusammenhang zwischen der Fragilität der Gläser und der Spannungsabhängigkeit könnte jedoch eine mögliche Erklärung hierfür bieten, da netzwerkbildende Glasbildner eine stärkere Abhängigkeit der elastischen Eigenschaften von der Fragilität zeigen. ${ }^{[97]}$ Festzuhalten ist, dass sowohl im fragilen als auch im starken Glasbildner eine klare Spannungsabhängigkeit der Glasübergangstemperatur zu beobachten ist. 
Analog zum PdCuSi wurde auch das Aktivierungsvolumen untersucht (siehe Abb. 3.36). Hierbei ist festzustellen, dass im Vergleich ein etwa doppelt so großes Aktivierungsvolumen beim $\mathrm{ZrAlCu}$ bestimmt werden konnte, wobei auch eine Abnahme mit der äußeren Spannung zu erkennen ist (siehe Abb. 3.37), welche analog zum PdCuSi mit einer Reduzierung der Barrierenhöhe innerhalb der PEL in Verbindung gebracht werden kann. Chen et al. berichten jedoch, dass das Aktivierungsvolumen, bestimmt für STZs, größer wird, wenn das Material eine höhere Fragilität aufweist. ${ }^{[66]}$ Bei den dafür durchgeführten Drucktests wurden aber deutlich größere Kräfte als bei den hier verwendeten Creep/Recoverymessungen benutzt. Außerdem ist zu beachten, dass die Versuche von Chen et al. bei Raumtemperatur und nicht in der Nähe von $\mathrm{T}_{G}$ durchgeführt wurden. Da die hier vorgestellten Experimente einen stärkeren Zusammenhang zwischen äußerer Spannung und dem Materialverhalten bei starken Gläsern als bei fragilen vermuten lassen, stehen die Ergebnisse von Chen et al. nicht im Widerspruch mit den hier diskutierten. Grundsätzlich wurden jedoch Aktivierungsvolumina auch für Zirkon basierte Gläser in derselben Größenordnung bestimmt. ${ }^{[66,67]}$

Oberhalb der Glasübergangstemperatur wurde auch der Versuch unternommen, ein Hochtemperatur-Barrierenlimit festzustellen. Analog zum PdCuSi wurde hierfür eine exponentielle Abnahme des Aktivierungsvolumens angenommen, da wegen der frühen Kristallisation (siehe Abb. 3.39) keine Aussage zum Materialverhalten gemacht werden kann. Es konnte ein Limit von 870 Atomen bestimmt werden, welches, wie auch das vorherig bestimmte Aktivierungsvolumen, etwa doppelt so groß wie das von PdCuSi ist.

\begin{tabular}{|c|c|c|c|}
\hline & $\mathrm{PdCuSi}$ & $\mathrm{ZrAlCu}$ & Faktor \\
\hline $\mathrm{T}_{G}$ Abnahme pro $\mathrm{MPa}[\mathrm{K}]$ & 1,3 & 2,4 & 1,8 \\
\hline $\begin{array}{l}\text { Aktivierungsvolumen }\left[\mathrm{nm}^{3}\right] \\
\text { (Atommenge) bei } 1 \mathrm{MPa}\end{array}$ & $8,6(570)$ & $18,2(1010)$ & $2,1(1,8)$ \\
\hline $\begin{array}{l}\text { Aktivierungsvolumen }\left[\mathrm{nm}^{3}\right] \\
\text { (Atommenge) Hochtemperatur- } \\
\text { Barrierenlimit }\end{array}$ & $7,5(490)$ & $15,7(870)$ & $2,1(1,8)$ \\
\hline Fragiliät m ${ }^{[14,111-115]}$ & $52,8-77,0$ & $36,4-38,5$ & $\frac{1}{1,5}-\frac{1}{2,1}$ \\
\hline
\end{tabular}

Tabelle 4.4: Vergleich der Spannungsabhängigkeit zwischen PdCuSi und ZrAlCu 
In Tabelle 4.4 sind die wichtigsten Parameter der beiden Glastypen gegenübergestellt. Dabei zeigt sich, dass sowohl in der Abnahme der Glasübergangstemperatur als auch bei den Größenordnungen des Aktivierungsvolumens ein Faktor zwischen den beiden Glastypen von 1,8 bzw. 2,1 vorliegt. Dies spiegelt sich auch beim Vergleich der Fragilitäten wieder, der den selben Zusammenhang reziprok $\left(\frac{1}{1,5}-\frac{1}{2,1}\right)$ aufweist. Dies lässt den Schluss zu, dass die Fragilität wichtig bei der Größenordnung der untersuchten Prozesse ist. Dabei scheint die Fragilität reziprok in den Zusammenhang einzugehen, je fragiler das Glas, desto geringer ist der Einfluss der äußeren Spannung. Möglich könnte hierfür z. B. die Eigenschaft von starken Gläsern sein, Netzwerke zu bilden, welche den stärkeren Einfluss auf kooperative Prozesse erklären kann. Allgemein kann gesagt werden, dass die Größe von STZs keine materialspezifische Konstante ist, aber die Größenordnung und das spannungsabhängige Verhalten der Prozesse an sich allgemeingültig zu sein scheinen. Sie besitzen aber keinen universellen Charakter, da sowohl die chemische Zusammensetzung als auch die Eigenschaft, Netzwerke auszubilden und die Fragilität einen Einfluss auf das Verhalten haben können.

\section{Fazit:}

Beim starken Glasbildner ZrAlCu konnte wie im fragilen Glasbildner PdCuSi sowohl die Glasübergangstemperatur als auch das Aktivierungsvolumen spannungsabhängig beobachtet werden. Dabei konnte eine Verringerung der Atommenge und der Temperatur bei größerer Spannung festgestellt werden. Im Vergleich zum PdCuSi wurde ein stärkerer Zusammenhang zur externen Spannung festgestellt, wobei das Aktivierungsvolumen in etwa doppelt so groß bestimmt wurde. Für die Spannungsabhängigkeit wird analog zum PdCuSi eine Verringerung der Barrierenhöhe innerhalb der PEL mit Zunahme der äußeren Spannung vorgeschlagen. Außerdem wurde ein Hochtemperatur-Barrierenlimit extrapoliert, welches etwa doppelt so groß wie im PdCuSi bestimmt wurde. Im Vergleich zwischen dem fragilen und dem straken Glasbildner wird vorgeschlagen, dass sowohl die Prozesse als auch die Größenordnung und die Spannungsabhängigkeit allgemeingültig sind, aber keinen universellen Charakter besitzen, da z. B. die Fragilität oder auch die chemische Komposition (Netzwerkbildner) einen Einfluss auf die Prozesse zu haben scheinen. 


\subsection{Relaxationen von wasserstoffbrückenbildenden Block-Copolymeren}

In dieser Arbeit wurden systematisch ABA-Block-Copolymere hinsichtlich des Einflusses von Wasserstoffbrückenbindungen auf ihre Relaxationsprozesse untersucht (siehe Kapitel 2.2 und 3.2). Hierzu wurde ein innerer Polymerblock aus Poly(tert.butylacrylat) (ptBA) von äußeren Blöcken mit einer statistischen Mischung aus $\mathrm{p} t \mathrm{BA}$ und Acrylsäure umgeben (siehe Kapitel 2.2). Um den Einfluss von Wasserstoffbrücken auf Relaxationen des inneren Blockes untersuchen zu können, wurde dieser bei konstanter Länge der äußeren Blöcke systematisch vergrößert.

Die so hergestellten Polymere wurden mittels DSC-, Röntgen- und DMA-Messungen sowohl auf ihre Amorphizität als auch auf ihre Relaxationsprozesse untersucht (siehe Kapitel 2). Beispielmessungen zu den DSC-Untersuchungen sind in Abbildung $3.40 \mathrm{zu}$ sehen, wobei sowohl ein Glasübergang als auch eine starke exotherme Reaktion oberhalb von $\mathrm{T}_{G}$ beobachtet werden kann. Dabei handelt es sich jedoch nicht um eine Kristallisation, wie sie bei metallischen Gläsern bekannt ist, was Röntgenspektren nach einer Auslagerung der Polymere oberhalb der Glasübergangstemperatur zeigen (siehe Abb. 3.43). Auch in DSC-Messungen ist nach einer Temperaturbehandlung noch eine Glasübergangstemperatur zu beobachten (siehe Abb. 3.41), diese liegt jedoch um $22 \mathrm{~K}$ höher als bei unbehandelten Proben (siehe Abb. 3.42). Die exotherme Reaktion ist aber nach der Temperaturbehandlung in DSC-Messungen deutlich geringer (siehe Abb. 3.41). Dies deutet auf eine chemische Reaktion hin, welche oberhalb der Glasübergangstemperatur auftritt. Dabei kann es sich z. B. um eine Säure-Base-Reaktion der Carbonylgruppen handeln, welche unter Wasserabscheidung die Polymere vernetzt. ${ }^{[118,119]}$ Hierfür spricht auch die deutlich höhere Glasübergangstemperatur, da die Polymere durch Vernetzungsreaktionen länger werden und somit eine deutlich höhere sterische Hinderung aufweisen. Eine weitere Möglichkeit wäre eine Abspaltung von Seitengruppen, was jedoch zu einer Erniedrigung von $\mathrm{T}_{G}$ führen sollte. ${ }^{[120]}$ Außerdem spricht das beobachtete Aufschäumen der Proben bei Temperaturbehandlung auch dafür, dass ein leicht flüchtiges Teilchen wie Wasser verdampft.

Bei den unbehandelten Proben kann systematisch sowohl in DSC- als auch in DMAMessungen bei Verlängerung des inneren Blockes eine annähernd lineare Abnahme der 
Glasübergangstemperatur beobachtet werden (siehe Abb. 3.42 und 3.49). Zur Bestimmung dient hierfür das Maximum des Relaxationspeaks bei Raumtemperatur. Diese Relaxation kann mit dem Weichwerden des Polymers in Verbindung gebracht werden, was auch in den Aufnahmen der Probenhöhen zu erkennen ist (siehe Abb. 3.46). Das beobachtete Zusammendrücken des Polymers erwies sich dabei als sehr gut reproduzierbar (siehe Abb. 3.47). Außerdem sind die Temperaturen des Erweichens in demselben Bereich wie die bestimmte Glasübergangstemperatur aus den DSC-Messungen.

Der Befund der kleiner werdenden Glasübergangstemperatur mit länger werdender Polymerkette ist zunächst überraschend, da in der Literatur eine höhere Glasübergangstemperatur wegen höherer sterischer Hinderung bei länger werdendem Polymer beschrieben wird. ${ }^{[121]}$ Für dieses Ergebnis bieten zwei mögliche Theorien eine Erklärung. Zum einen ist es denkbar, dass eine Mischung der Glasübergangstemperaturen von ptBA (abhängig von der Länge der Polymere $263 \mathrm{~K}-343 \mathrm{~K}^{[122,123]}$ ) und Acrylsäure (380 $\mathrm{K}^{[117]}$ ) beobachtet wird. Dadurch, dass der Anteil der Acrylsäure mit zunehmendem B-Block kleiner wird, wird auch die Glasübergangstemperatur gesenkt und nähert sich dem des ptBA an. Gegen diese Hypothese spricht jedoch, dass eine Glasübergangstemperatur von reinem ptBA bei $305 \mathrm{~K}$ bestimmt wurde (bei einer Molmasse von $18300 \mathrm{~g} / \mathrm{mol}$ ). Diese entspricht dem des ABA - Polymers mit einem inneren Block von $5504 \mathrm{~g} / \mathrm{mol}$ und äußeren Blöcken von $11300 \mathrm{~g} / \mathrm{mol}$ (siehe Abb. 3.48). Bei Polymeren mit längerem inneren Block und einer größeren Gesamtmolekularmasse wurden jedoch Glasübergangstemperaturen von unter $300 \mathrm{~K}$ gemessen (siehe Abb. 3.47). Somit liegt $\mathrm{T}_{G}$ unterhalb des reinen ptBA. Außerdem konnte bei Messungen mit gleichlangem B-Block und unterschiedlichen langen A-Blöcken (siehe Abb. 3.45) nachgewiesen werden, dass sich die Glasübergangstemperatur nicht verschiebt, was ebenfalls gegen eine gemischte Glasübergangstemperatur spricht.

Eine weitere Möglichkeit zur Erklärung dieses Befundes ist, dass eine Glasübergangstemperatur des inneren Blockes bestimmt wurde. Dadurch, dass der innere Block durch die außen gelegenen Wasserstoffbrückenbindungen fixiert wird, kann ein Erweichen leichter geschehen, wenn der Block länger wird. Es entsteht mehr Raum, der für Relaxationen benutzt werden kann. Bei sehr kleinen Blöcken ist das Polymer insgesamt sehr starr und zeigt dadurch eine höhere Glasübergangstemperatur als reines $\mathrm{p} t \mathrm{BA}$. Wird jedoch der innere Block vergrößert und erhält dadurch mehr Raum für Bewegungen, kann das 
Erweichen bei tieferen Temperaturen stattfinden. Da der innere Block kleiner ist als das untersuchte reine $\mathrm{p} t \mathrm{BA}$, kann auch die geringere Glasübergangstemperatur erklärt werden.

Außer dem Relaxationspeak bei Raumtemperatur, welcher über DMA-Messungen aufgenommen und als die Glasübergangstemperatur identifiziert werden konnte, wurden weitere Maxima in den Verlustspektren beobachtet (siehe Abb. 3.44). Bei tiefen Temperaturen um $150 \mathrm{~K}$ kann ein $\gamma$ - Peak identifiziert werden. Dieser wird in der Literatur als eine Wellen- oder auch Kurbelwellenbewegung der Gerüstkette der Alkylgruppen interpretiert (siehe auch Kapitel 1.5). ${ }^{[18,116]}$

Oberhalb der Glasübergangstemperatur kann ein weiterer stark ausgeprägter Verlustprozess beobachtet werden (siehe Abb. 3.44), welcher mit einem weiteren Viskositätsverlust in Verbindung gebracht werden kann (siehe Abb. 3.47). Es konnte hierbei ein Zusammenhang mit der Länge des A-Blockes hergestellt werden (siehe Abb. 3.46). Außerdem konnte in reinem $\mathrm{p} t \mathrm{BA}$ keine starke Viskositätsveränderung oberhalb von $\mathrm{T}_{G}$ beobachtet werden (siehe Abb. 3.48), was ebenfalls einen Rückschluss auf einen Bezug zum A-Block zulässt. Der Temperaturbereich dieses Verlustprozesses passt sehr gut zu der in den DSC-Messungen gefundenen exothermen Reaktion, so dass hier eine Beziehung zum beobachteten Relaxationsvorgang möglich ist. Als Erklärung hierfür kommen nur die durch Acrylsäure hervorgerufenen Wasserstoffbrücken in Frage, da diese in reinem p $t$ BA fehlen. Dies bedeutet jedoch auch, dass die Wasserstoffbrücken oberhalb der Glasübergangstemperatur für eine Stabilisierung des Systems sorgen müssen, da in einem Bereich von etwa $50 \mathrm{~K}\left(\mathrm{~T}_{G}\right.$ bis $\left.\mathrm{T}_{G}+50 \mathrm{~K}\right)$ die Probenhöhe konstant bleibt, bis das Polymer durch ein weiteres Erweichen aus der Halterung gedrückt wird. Zwei mögliche Reaktionen könnten diese Viskositätsänderung hervorrufen. Zum einen könnten die Wasserstoffbrücken bei dieser Temperatur aufgebrochen werden, was zu weiterem Erweichen führen würde. Dies sollte aber eine endotherme Reaktion darstellen und ist daher höchstwahrscheinlich nur eine Begleiterscheinung der zuvor diskutierten chemischen Reaktion. Dadurch, dass eine Säure-Base-Reaktion ablaufen könnte, würde das entstehende Wasser ein Erweichen des Polymers hervorrufen, da es mit den restlichen $\mathrm{OH}-\mathrm{Gruppen}$ wechselwirkt und somit als Lösungsmittel für das Polymer fungiert. Da jedoch eine Verschiebung dieser Reaktion zu höheren Temperaturen mit längerem A-Block festgestellt werden konnte (siehe Abb. 3.50), scheinen mehr Wasserstoffbrücken dieser Reaktion entgegenzuwirken. 
Dies bedeutet, dass, bevor die Reaktion beginnen kann, genügend Wasserstoffbrücken aufgebrochen werden müssen, damit ausreichend Reaktionspartner vorliegen. Eine weitere Möglichkeit ist, dass eine Glasübergangstemperatur für die äußeren Blöcke zu sehen ist. Der beobachtete Temperaturbereich von etwa $390 \mathrm{~K}$ passt zu der in der Literatur beschriebenen Glasübergangstemperatur von Acrylsäure von etwa 380 K. ${ }^{[117]}$ Jedoch sollte, da eine Mischung aus ptBA und Acrylsäure vorliegt, die Glasübergangstemperatur unterhalb der von reiner Acrylsäure liegen.

Eine weitere sekundäre Relaxation im Temperaturbereich zwischen dem $\gamma$ - Peak und der Glasübergangstemperatur konnte analog zu den Ergebnissen von Jörg Hachenberg ${ }^{[14]}$ festgestellt werden (siehe Abb. 3.44 und 3.51), welche in reinem ptBA nicht zu sehen ist. ${ }^{[16]}$ Dabei konnte durch Wiederholungen der Messungen mit verschiedenen Proben die Relaxation gut reproduzierbar beobachtet werden (siehe Abb. 3.51). Bei der Untersuchung der zugehörigen Temperatur konnte ein Zusammenhang zum Molekulargewicht des inneren Blockes festgestellt werden (siehe Abb. 3.52). Dabei verschiebt sich das Maximum mit länger werdendem inneren Block zu höheren Temperaturen und zeigt eine klare lineare Korrelation.

Analog zu den Arbeiten von Jörg Hachenberg ${ }^{[14]}$ wird ein Wasserstoffbrücken induziertes Chemical Confinement vorgeschlagen, um diesen Befund zu erklären. Dabei werden die äußeren Blöcke über die Wasserstoffbrücken fixiert, so dass eine neue Längeneinheit mit der Länge des inneren Blockes entsteht. Diese kann durch Temperaturerhöhung zu Kurbelwellenbewegungen angeregt werden, wobei mehr Energie für längere Einheiten benötigt wird.

Interessanterweise konnte Cerrada et al. ein ähnliches Verlustmaximum bei Copolymeren aus Poly(tert.-butylacrylat) mit Styrolseitengruppen beobachten, welches ebenfalls zwischen dem $\gamma$-Peak und dem Glasübergang liegt und im reinen $\mathrm{p} t \mathrm{BA}$ nicht zu finden ist. ${ }^{[116]}$ Das Maximum verschiebt sich bei diesen Versuchen zu geringeren Temperaturen, wenn die Seitengruppenanzahl zunimmt. Erklärt wird dies über eine Bewegung der -COO-tert-butyl-Gruppen, wobei eine größere sterische Hinderung bei Zunahme an Seitenarmen vorliegt.

Es kann jedoch auch angenommen werden, dass die Seitengruppen über intra- und intermolekulare Wechselwirkungen ein Chemical Confinement hervorrufen, welche in der Polymerkette, analog zu den in Kapitel 3.2 vorgestellten Ergebnissen, eine neue 
Längeneinheit schaffen. Eine Erhöhung der Anzahl an Seitengruppen schafft kleinere Segmente in der Hauptkette und sorgt somit für eine niedrigere Temperatur des neuen Relaxationsmaximums. Diese Interpretation stützt die These des Wasserstoffbrücken induzierten Chemical Confinements, da in dieser Arbeit mit Polymeren ohne lange Seitenketten gearbeitet wurde.

\section{Fazit:}

In wasserstoffbrückenbildenden ABA-Block-Copolymeren konnten verschiedene Relaxationsprozesse sowohl oberhalb als auch unterhalb der Glasübergangstemperatur untersucht werden. Dabei wurde im Temperaturbereich zwischen $150 \mathrm{~K}$ und $200 \mathrm{~K}$ ein $\gamma$ - Peak identifiziert. Die Glasübergangstemperatur wurde in der Nähe zur Raumtemperatur gefunden und einem Erweichen des inneren Blockes zugeordnet. Dabei wurde die Glasübergangstemperatur mit länger werdendem Polymer kleiner. Eine mögliche Erklärung hierfür ist, dass sich die Gesamtmischung zwischen ptBA und Acrylsäure verändert oder dass durch die Fixierung der äußeren Blöcke durch Wasserstoffbrückenbindungen eine separierte Glasübergangstemperatur des B-Blocks beobachtet wird. Zwischen dem $\gamma$ - Peak und dem Glasübergang wurde ein zusätzliches Relaxationsmaximum gefunden, das einem Wasserstoffbrücken induzierten Chemical Confinement zugeschrieben wird, welches eine neue Längeneinheit in der Gerüstkette schafft. Außerdem wurde eine weitere Viskositätsänderung oberhalb der Glasübergangstemperatur beobachtet, welche in reinem ptBA nicht zu sehen ist. Die Block-Copolymere werden über die Wasserstoffbrücken in einem Temperaturbereich von etwa $50 \mathrm{~K}$ überhalb von $\mathrm{T}_{G}$ stabilisiert, bis ein endgültiges Erweichen vorliegt. Dabei ist ein Aufbrechen der Wasserstoffbrücken mit anschließender Säure-Base-Reaktion als Erklärung möglich, da durch das hierbei entstehende Wasser, welches als Lösungsmittel dient, ein weiteres Erweichen des Polymers erzeugt werden kann. 


\section{Zusammenfassung}

Relaxationsprozesse von komplexen Fluiden stellen einen bedeutenden Aspekt zum Verständnis sowohl von metallischen Gläsern als auch von Polymeren dar. Deshalb ist die Interpretation der makroskopischen Eigenschaften hinsichtlich ihrer mikroskopischen Vorgänge ein sehr wichtiges Thema in der aktuellen Forschung. Dabei ist das Verständnis des Übergangs vom festen, amorphen Zustand in die unterkühlte Schmelze fundamental. Hierzu wurde in dieser Arbeit das Elastizitätsverhalten metallischer Glasbildner untersucht. Zum einen wurde die Heizrate bei dynamisch mechanischen Messungen systematisch verringert, um Veränderungen in den mechanischen Eigenschaften zu studieren und durch periodisches Aufheizen und Abkühlen Alterungsprozesse zu untersuchen. Zum anderen wurde der Übergang in die unterkühlte Schmelze spannungsabhängig sowohl am fragilen Glasbildner $\mathrm{Pd}_{77,5} \mathrm{Cu}_{6,0} \mathrm{Si}_{16,5}$ als auch am starken $\mathrm{Zr}_{65} \mathrm{Al}_{7,5} \mathrm{Cu}_{27,5}$ beobachtet, um die von Johnson und Samwer vorgeschlagene spannungsabhängige potentielle Energielandschaft genauer zu beschreiben.

Weiterhin wurden systematisch ABA-Block-Copolymere hinsichtlich des Einflusses von Wasserstoffbrückenbindungen auf ihre Relaxationsprozesse untersucht. Hierzu wurde ein innerer Polymerblock aus Poly(tert.-butylacrylat) (ptBA) von äußeren Blöcken mit einer statistischen Mischung aus ptBA und Acrylsäure umgeben.

\section{Metallische Gläser}

Bei langsamen Heizraten konnte ein bisher nicht bekanntes Dämpfungsmaximum unterhalb der Glasübergangstemperatur festgestellt werden, welches möglicherweise einem strukturellen Übergang entspricht und durch ein Aufweichen der Matrix eine erleichterte Anregung der langsamen $\beta$ - Relaxation hervorruft. Dies scheint auch in Verbindung mit der heizratenabhängigen Glasübergangstemperatur zu stehen, welche sich durch Anregungen innerhalb der potentiellen Energielandschaft (PEL) beschreiben lässt.

Eine sehr ähnliche Auswirkung von Temperatur und Spannung auf das mechanische 
Verhalten von metallischen Gläsern konnte bei der Untersuchung von SpannungsDehnungskurven beobachtet werden. Dabei bewirkte eine Verlangsamung der Spannungsänderung eine Verschiebung des Überganges vom elastischen zum plastischen Bereich zu kleineren Spannungen. Dieser Effekt wurde ebenfalls bei einer Erhöhung der Temperatur festgestellt, wobei der Übergang zum nichtlinearen Bereich systematisch mit einer Erhöhung der Temperatur untersucht wurde. So konnten die Veränderungen im elastischen Verhalten sowohl auf eine thermische Anregung von energetisch höher gelegenen Subbasins als auch auf eine Erniedrigung der Barrierenhöhe innerhalb der PEL zurückgeführt werden. Bei sehr langsamen Dehnungsraten konnte auch innerhalb des linearen Bereiches der Spannungs-Dehnungskurve ein Indiz für nichtlineare Reaktionen gefunden werden.

Weitere Untersuchungen mit Hilfe von Creep/Recoverymessungen konnten zeigen, dass sowohl eine Erhöhung der Temperatur als auch eine äußere Spannung das Dämpfungsverhalten von amorphen $\mathrm{PdCuSi}$ unterhalb der Glasübergangstemperatur beeinflusst. Dabei wird das Material weicher, je höher die Temperatur oder die Spannung ist. Es konnte außerdem durch Creep/Recoverymessungen mit einer konstanten Heizrate sowohl ein spannungsabhängiges Elastizitätsverhalten nahe der Glasübergangstemperatur als auch eine spannungsabhängige Abnahme der Glasübergangstemperatur bestimmt werden. Erstmals wurde das Aktivierungsvolumen für Scher-Transformations-Zonen über diese Messmethode bestimmt, welches ebenfalls als spannungsabhängig bis oberhalb der Glasübergangstemperatur beobachtet wurde. Ein Vergleich zwischen dem fragilen Glasbildner $\mathrm{Pd}_{77,5} \mathrm{Cu}_{6,0} \mathrm{Si}_{16,5}$ und dem starken $\mathrm{Zr}_{65,0} \mathrm{Al}_{7,5} \mathrm{Cu}_{27,5}$ zeigte dabei vergleichbare Zusammenhänge zwischen Temperatur und Spannung, wobei jedoch eine stärkere Spannungsabhängigkeit beim $\mathrm{ZrAlCu}$ gefunden wurde. Außerdem konnte ein etwa doppelt so großes Aktivierungsvolumen für den starken Glasbildner berechnet werden. Im Vergleich zwischen dem fragilen und dem starken Glasbildner wird vorgeschlagen, dass sowohl die Prozesse als auch die Größenordnung und die Spannungsabhängigkeit allgemeingültig sind, aber keinen universellen Charakter besitzen, da z. B. die Fragilität oder auch die chemische Komposition (Netzwerkbildner) einen Einfluss auf die Prozesse zu haben scheinen. 


\section{Block-Copolymere}

In wasserstoffbrückenbildenden ABA-Block-Copolymeren konnten verschiedene Relaxationsprozesse sowohl oberhalb als auch unterhalb der Glasübergangstemperatur untersucht werden. Hierzu wurde ein innerer Polymerblock aus Poly(tert.-butylacrylat) (ptBA) von äußeren Blöcken mit einer statistischen Mischung aus ptBA und Acrylsäure umgeben. Dabei wurde im Temperaturbereich zwischen $150 \mathrm{~K}$ und $200 \mathrm{~K}$ ein $\gamma$ - Peak identifiziert. Die Glasübergangstemperatur wurde in der Nähe der Raumtemperatur gefunden und einem Erweichen des inneren Blockes zugeordnet. Zwischen dem $\gamma$ - Peak und dem Glasübergang wurde ein zusätzliches Relaxationsmaximum beobachtet, das einem Wasserstoffbrücken induzierten Chemical Confinement zugeschrieben wird, welches eine neue Längeneinheit in der Gerüstkette schafft. Eine Stabilisierung des Polymerverhaltens mit einer weiteren Viskositätsänderung oberhalb der Glasübergangstemperatur wurde beobachtet, wobei ein Aufbrechen der Wasserstoffbrücken mit anschließender SäureBase-Reaktion als Erklärung dient, da durch das hierbei entstehende Wasser, welches als Lösungsmittel fungiert, ein weiteres Erweichen des Polymers erzeugt werden kann. 



\section{Anhang}

\section{Quelltext zur Datenanalyse}

import table 2

class Chunk(table2.Table):

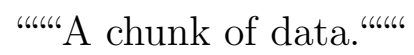

def_init_(self, spawn_table):

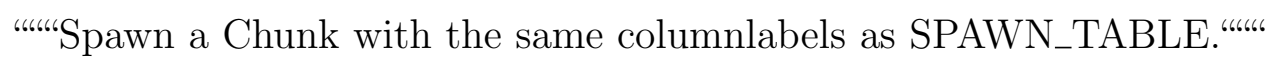

table2.Table._init__(self)

self.columnlabels $=$ spawn_table.columnlabels

def add_row(self, row_data):

«แแAppend a row ROW_DATA to this chunk.

self.put_row_list(self.shape[1], row_data)

import table2

import table2.fileio

import moritz.chunk

class File:

«แแThis file class converts a string into Chunks. It allows for storing of the data.

def_init_(self, table, indicator_column): 
“แ'Initialise from loaded table TABLE and use INDICATOR_COLUMN to distinguish between adjacent Chunks.““แ

self.table $=$ table

\# Load chunks ...

self.chunks $=[]$

\# Initialise state.

current_chunk $=$ moritz.chunk.Chunk(table)

self.chunks.append(current_chunk)

current_indicator $=$ table[indicator_column, 0$]$

\# Iterate through the table.

for rowidx in xrange(0, table.shape[1]):

indicator $=$ table[indicator_column, rowidx]

row_data $=$ table.get_row_list $($ rowidx $)$

if indicator $==$ current_indicator:

\# Same chunk.

current_chunk.add_row(row_data)

else:

\# Current chunk finished, start new one.

current_chunk $=$ moritz.chunk.Chunk(table)

self.chunks.append(current_chunk)

current_chunk.add_row(row_data) 
current_indicator $=$ indicator

def tofile_edges(self, filename, skip):

«แ' Store the data of the rising and falling edges in file FILENAME.

The resulting table will have four groups of columns:

1. The last datum from the first chunk.

2. The SKIPth datum from the following chunk.

3. The last datum from the same chunk as in (2.).

4. The SKIPth datum from the next following chunk.

The last datum from the next following chunk goes into (1.).

If the file ends with an incomplete line, this line will be left out. $\cdots u \omega$

\# Prepare the columns of the output table ...

output_table $=$ table2.Table ()

\# Repeat the columns four times.

width $=$ self.table.shape[0]

for repeatidx in xrange $(0,4)$ :

\# Iterate through all labels.

for (label, column) in self.table.columnlabels.items():

output_table.label_column(

column + repeatidx * width,

label $+\operatorname{str}($ repeatidx +1$))$

\# For each data row, three chunks are needed: One for (1.), one for

\# (2.) \& (3.), and one for (4.). The chunk needed for (4.) is also

\# needed for (1.) in the following line. Some examples:

\# Number of Chunks Maximum Row Index and Chunks used for each row 
\# $000:[]$

\# 10 : 0 : []

\# 20 : []

\# $31:[(0,1,2)]$

\# $41:[(0,1,2)]$

\# 52 : [(0,1,2), $(2,3,4)]$

\# 62 : [(0,1,2), $(2,3,4)]$

\# 73 : [(0,1,2), $(2,3,4),(4,5,6)]$

\# From this examples, we see:

maximum_rowidx $=($ len $($ self.chunks $)-1) / 2$

\# We see, that this yields -1 for $\operatorname{len}()==0$, but that isn't a

\# problem.

\# Iterate though all output lines ...

for rowidx in xrange(0, maximum_rowidx):

\# Extract the relevant chunks.

chunk_1st $=$ self.chunks[rowidx $* 2]$

chunk_2nd $=$ self.chunks[rowidx $* 2+1]$

chunk_3rd $=$ self.chunks[rowidx $* 2+2]$

if chunk_1st.shape[1] $<=$ skip or

chunk_2nd.shape[1] $<=$ skip or

chunk_3rd.shape[1] $<=$ skip:

print "Chunks for data row idx \%s too short! Chunk lengthes: (\%d, \%d, \%d)" \% (rowidx, chunk_1st.shape[1], chunk_2nd.shape[1], chunk_3rd.shape[1])

else:

\# Calculate the row data.

row_data $=\backslash$

chunk_1st.get_row_list(-1) + । 
chunk_2nd.get_row_list(skip - 1) + ।

chunk_2nd.get_row_list $(-1)+1$

chunk_3rd.get_row_list(skip - 1)

\# Put the data line

output_table.put_row_list(rowidx, row_data)

\# Write the output file.

table2.fileio.tofile(filename, output_table,

with_columnlabels $=$ True,

$\left.\operatorname{sep}=' \backslash t^{\prime}\right)$

import optparse

import sys

\# Create parser ...

parser $=$ optparse.OptionParser (

description = 'This program converts files using "," as decimal separator to files using "." as decimal separator. All occurences of "," will be replaced by ".". The file will be modified in place.

parser.set_defaults $($ inoutfile $=$ None $)$

parser.add_option('-f', '-input-output', dest = 'inoutfile',

action $=$ 'store',

help $=$ 'The file to convert. It will be modified IN PLACE. This means, no copy will be created.')

(options, args) = parser.parse_args $($ )

\# Check options ... 
if options.inoutfile is None:

print "INOUTFILE missing. See -help."

sys.exit $(2)$

\# Convert file ...

\# Read file data.

try:

infile $=$ open (options.inoutfile $)$

except Exception, exc:

print "Could not open file for reading:", exc

sys.exit(1)

content $=$ infile.read ()

infile.close ()

\# Convert file data.

content $=$ content.replace(',', '.' $)$

\# Write file data.

try:

outfile $=$ open(options.inoutfile, 'w')

except Exception, exc:

print "Could not open file for writing:", exc

sys.exit(1)

outfile.write(content)

outfile.close()

\# Done. 
import optparse

import sys

import table2.fileio

import moritz.file

\# Create the parser ...

parser $=$ optparse.OptionParser (

description $=$ "'This program extracts the data of the rising and falling edges. The resulting table will have four groups of columns: 1 . The last datum from the first chunk. 2. The SKIPth datum from the following chunk. 3. The last datum from the same chunk as in (2.). 4. The SKIPth datum from the next following chunk. The last datum from the next following chunk goes into (1.). If the file ends with an incomplete line, this line will be left out."')

parser.set_defaults (

infile $=$ None

outfile $=$ None,

skip $=15$,

indicator_column $=$ 'StaticForce')

parser.add_option('-i', '-input-file', dest = 'infile',

help = 'The input file. A table of arbitrary size, with "." as arithmetic separator, and Tab as cell separator.')

parser.add_option('-o', '-output-file', dest = 'outfile',

help = 'The output file.')

parser.add_option('-s', '-offset', '-skip', dest = 'skip',

type = 'int',

help = 'The skip used when extracting data. Default is 15.') 
parser.add_option('-c', '-indicator_column', dest = 'indicator_column', help $=$ 'The column used to indicate chunks. Chunks are groups of adjacent rows with the same value in this column. Default is "StaticForce". If the argument is interpretable as an integer, it will be used as the zero-based index of the column.')

(options, args $)=$ parser.parse_args ()

\# Check arguments ...

if None in (options.infile, options.outfile):

print "Some file argument missing. Check -help."

sys.exit $(2)$

try:

indicator_column $=$ eval(options.indicator_column)

except:

indicator_column $=$ options.indicator_column

\# Load table ..

print "Loading file ..."

table $=$ table2.fileio.fromfile(options.infile, with_columnlabels $=$ True, sep $=$ ' $\backslash \mathrm{t}$ ')

\# Analyse data ...

print "Analysing data ..."

file $=$ moritz.file.File(table, indicator_column)

\# Write data out ... 
print "Writing data ..."

file.tofile_edges(options.outfile, options.skip)

from distutils.core import setup

import py2exe

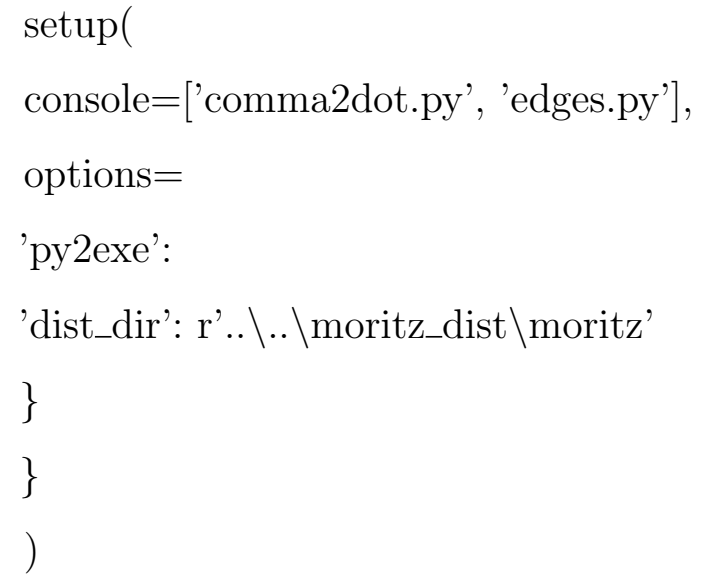





\section{Literaturverzeichnis}

[1] K.H. Wedepohl, Glas in Antike und Mittelalter: Geschichte eines Werkstoffs, 2003, E. Schweizerbart'sche

[2] M.A. Bezborodov, Chemie und Technologie der antiken und mittelalterlichen Gläser, 1975, P. von Zabern

[3] E. Baumgartner, I. Krueger, Phönix aus Sand und Asche: Glas des Mittelalters, 1988, Klinkhardt \& Biermann

[4] M. Merkel, K.H. Thomas, Taschenbuch der Werkstoffe, 1994, Fachbuchverlag Leipzig

[5] S.G. Klose, Phasenstabilität, thermodynamische und mikromechanische Eigenschaften der metallischen Massivgläser ZrTiNiCuBe und AuPbBe, 1995, Herbert UTZ Verlag, München

[6] E.J. Donth, Glasübergang, 1981, Akademie-Verlag, Berlin

[7] W.L. Johnson, MRS Bulletin (USA), 1999, 24, 10, 42-56

[8] S.R. Elliot, Physics of amorphous materials, 1990, second edition, Longman Scientific \& Technical

[9] J. Zarzycki, Glasses and the vitreous state, 1991, Cambridge University Press

[10] P.G. Debenedetti, F.H. Stillinger, Nature, 2001, 410, 259, London

[11] R. Rambousky, Dynamisch-mechanische Eigenschaften neuer ternärer und mehrkomponentiger Gläser, 1994, Diplomarbeit, Augsburg 
[12] M. Weiß, Mechanische Eigenschaften und thermische Stabilität tief unterkühlter Metallschmelzen im Bereich der Glastemperatur, 1998, Dissertation, Augsburg

[13] P. Rösner, Mechanische Relaxation in komplexen Fluiden, 2004, Dissertation, Göttingen

[14] J. Hachenberg, Sekundäre Relaxationen in amorphen Festkörpern, 2006, Dissertation, Göttingen

[15] R.P. Feynman, R.B. Leighton, M. Sands, The Feynman lectures on physics, Band I-III, 6. Auflage, 1977, Addison Wesley

[16] L.D. Landau, E.M. Lifschitz, Lehrbuch der theoretischen Physik, Band VII, Elastizitätstheorie, 7. Auflage, 1991, Akademie Verlag Berlin

[17] C.A. Angell, J. of Non-Cryst. Sol., 1988, 102, 205

[18] D.S. Jones, Inter. Jour. of Pharm., 1999, 179, 167-178

[19] K.P. Menard, Dynamic mechanical analysis: a practical introduction, 1999, CRC Press

[20] Prof. Dr. Helmut Föll, Einführung in die Materialwissenschaft I, 2007, Christian-Albrechts-Universität Kiel http : //www.tf.uni - kiel.de/matwis/amat/mw $1_{g} e /$

[21] A. Kahl, Ultraschallspektroskopie an dem amorphen System $P d_{40} N i_{40} P_{20}$, 2006, Diplomarbeit, Göttingen

[22] T. Koeppe, Temperaturabhängigkeit der elastischen Konstanten für das metallische Glas $P d_{40} N i_{40} P_{20}, 2007$, Diplomarbeit, Göttingen

[23] U. Rabe, W. Arnold, Appl. Phys. Lett., 1994, 64, 1493-1495

[24] M. Kopycinska-Müller, A. Caron, S. Hirsekorn, U. Rabe, H. Natter, R. Hempelmann, R. Birringer, W. Arnold, Z. Phys. Chem., 2008, 222, $471-498$ 
[25] C. Vree, Topographie, Struktur und Dynamik thermisch aufgedampfter Polymerfilme, 2009, Dissertation, Göttingen

[26] R.N. Kleiman, G.K. Kaminsky, J.D. Reppy, R. Pindak, D.J. Bishop, Rev. Sci. Instrum., 1985, 56, 11, 2088-2091

[27] D. Bedorf, Mechanische Spektroskopie an metallischen Gläsern in reduzierter Dimensionalität, 2009, Dissertation, Göttingen

[28] H. Vogel, Phys. Z., 1921, 22, 645

[29] G.S. Fulcher, J. Am. Ceram. Soc., 1923, 8, 229

[30] G. Tammann, W. Hesse, Z. Anorg. Allg. Chem., 1926, 156, 245

[31] T. Hecksher, A.I. Nielsen, N.B. Olsen, J.C. Dyre, Nature Physics, 2008, $4,9,737-741$

[32] P. Lunkenheimer, U. Schneider, R. Brand, A. Loidl, Contemp. Phys., 2000, 41, 1, 15

[33] B. Schrader, Infrared and Raman Spectroscopy: Methods and Applications, 1995, $\mathrm{VCH}$

[34] A.I. Chumakov, I. Sergueev, U. van Bürck, W. Schirmacher, T. Asthalter, R. Rüffer, O. Leupold, W. Petry, Phys. Rev. Lett., 2004, 92, 245508

[35] C.A. Angell, J. Phys.: Cond. Mat., 2004, 16, 5153

[36] W. Götze, Cond. Mat. Phys., 1998, 1, 4, 873

[37] J.C. Dyre, Phys. Rev. E, 2005, 72, 011501

[38] A. Kudlik, S. Benkhof, T. Blochowicz, C. Tschirwitz, E. Rössler, J. Mol. Struct., 1999, 479, 201

[39] M.D. Ediger, C.A. Angell, S.R. Nagel, J. Phys. Chem., 1996, 100, 13200

[40] E.J. Donth, The glass transition - Relaxation dynamics in liquids and disordered materials, 2001, Springer Verlag 
[41] A. Meyer, R. Busch, H. Schober, Phys. Rev. Lett., 1999, 83, 5027

[42] J. Hachenberg, K. Samwer, Journal of Non-Crystalline Solids, 2006, 352, 42-49, 5110-5113

[43] H. Schober, Physica, 1993, (from Miracle MRS Bull 2007)

[44] J.D. Eshelby, Proc. R. Soc. A, 1957, 241, 376

[45] D. Bedorf, K. Samwer, Journal of Non-Crystalline Solids, 2010, 356, 6-8, 340-343

[46] M. Neudecker, S.G. Mayr, Acta Mater., 2009, 57, 1437

[47] W.L. Johnson, K. Samwer, Physical Review Letters, 2005, 95, 195501

[48] M.D. Demetriou, J.S. Harmon, M. Tao, G. Duan, K. Samwer, W.L. Johnson, Phys. Rev. Lett., 2006, 97, 089901

[49] W.L. Johnson, M.D. Demetriou, J.S. Harmon, M.L. Lind, K. Samwer, MRS Bulletin, 2007, 32, 644

[50] J.S. Harmon, M.D. Demetriou, W.L. Johnson, K. Samwer, Physical Review Letters, 2007, 99, 135502

[51] A.S. Argon, Acta Metall., 1979, 27, 47

[52] A.S. Argon, H.Y. Kuo, J. Non-Cryst. Solids, 1980, 37, 241

[53] A.S. Argon, L.T. Shi, Acta Metall., 1983, 31, 499

[54] L.T. Shi, A.S. Argon, H.Y. Kuo, Scr. Metall., 1983, 17, 1015

[55] J.D. Eshelby, Proc. R. Soc. A, 1959, 252, 1271

[56] G.P. Johari, M. Goldstein, J. Chem. Phys., 1970, 53, 2372

[57] P. Lunkenheimer, R. Wehn, U. Schneider, A. Loidl, Phys. Rev. Lett., 2005, 95, 055702

[58] K. Chen, K.S. Schweizer, Phys. Rev. Lett., 2007, 98, 16, 167802 
[59] C.A. Angell, K.L. Ngai, G.B. McKenna, P.F. McMillan, S.W. Martin, J. Appl. Phys., 2000, 88, 3113

[60] G.B. McKenna, J. Phys. Condens. Matter, 2003, 15, 737

[61] A. Kahl, T. Koeppe, D. Bedorf, R. Richert, M.L. Lind, M.D. Demetriou, W.L. Johnson, W. Arnold, K. Samwer, Appl. Phys. Lett., 2009, 95, 201903

[62] P. Rösner, K. Samwer, R.O. Pohl, S. Schneider, Rev. Sci. Instrum., 2003, 74,3395

[63] J. Frenkel, Z. Phys., 1926, 37, 572

[64] H. Sillescu, J. Non-Cryst. Solids, 1999, 243, 81-108

[65] C.A. Angell, K.L. Ngami, J. Kieffer, T. Egami, G.U. Nienhaus, Structure and Dynamics of Glasses and Glass Formers, 1997, MRS Symposium Proceedings, Volume 455, Materials Research Society

[66] D. Pan, A. Inoue, T. Sakurai, M.W. Chen, PNAS, 2008, 0105, 39, 14769 - 14772

[67] D. Pan, Y. Yokoyama, T. Fujita, Y.H. Liu, S. Kohara, A. Inoue, M.W. Chen, Applied Physics Letters, 2009, 95, 141909

[68] H. Teichler, Phys. Rev. E, 2005, 71, 031505

[69] I. Ladadwa, H. Teichler, Phys. Rev. E, 2008, 78, 041503

[70] G. Adam, J.H. Gibbs, J. Chem. Phys., 1965, 43, 139

[71] M. Zink, K. Samwer, W.L. Johnson, S.G. Mayr, Phys. Rev. B, 2006, 73, 172203

[72] S.G. Mayr, Phys. Rev. Lett., 2006, 97, 195501

[73] Y. Champion, S. Nowak, Materials Science Forum, 2008, 584-586, 399404 
[74] N.G. McCrum, B.E. Read, G. Williams, Anelastic and dielectric effects in polymeric solids, 1967, John Wiley \& Sons

[75] L.H. Sperling, Introduction to physical polymerscience, 1992, second edition, Wiley-Interscience

[76] H. Jones, Treatise on materials science and technology: Volume 20 Ultrarapid quenching of liquid alloys, 1981, Academic press

[77] A.L. Greer, Science, New Series, 1995, 267, 5206, 1947-1953

[78] B.D. Cullity, Elements of X-Ray Diffraction, 1978, second edition, Addison-Wesley

[79] Perkin-Elmer, User Manual 7 Series / Unix DMA 7/DMA 7e, 1994, manual version F, Perkin-Elmer

[80] J. Chiefari, Y.K. Chong, F. Ercole, J. Krstina, J. Jeffery, T.P.T. Le, R.T.A. Mayadunne, G.F. Meijs, C.L. Moad, G. Moad, E. Rizzardo, S.H. Thang, Macromolecules, 1998, 31, 5559

[81] R. Rotzoll, D.H. Nguyen, P. Vana, Macromolecular Symposia, 2009, $275-276,1$

[82] R. Rotzoll, P. Vana, Journal of Polymer Science Part A: Polymer Chemistry, 2008, 46, 7656

[83] D. Boschmann, M. Mänz, A.C. Pöppler, N. Sörensen, P. Vana, Journal of Polymer Science Part A: Polymer Chemistry, 2008, 46, 7280

[84] M. Schwabe, R. Rotzoll, S. Küchemann, K. Nadimpalli, P. Vana, K. Samwer, Macromolecular Chemistry and Physics, 2010, 211, 15, 1673 $-1677$

[85] J. Hachenberg, D. Bedorf, K. Samwer, R. Richert, A. Kahl, M.D. Demetriou, W.L. Johnson, Appl. Phys. Lett., 2008, 92, 131911

[86] J. Jäckle, Rep. Prog. Phys., 1986, 49, 171 
[87] A.P. Sokolov, A. Kisliuk, D. Quitmann, A. Kudlik, E. Rössler, J. NonCryst. Solids, 1994, 172-174, 138-153

[88] S.M. Chathoth, Structure and Dynamics of Al and Ni Based Melts Studied by Inelastic Neutron Scattering., 2005, Dissertation, Technische Universität München

[89] R. Richert, K. Samwer, New J. Phys., 2007, 9, 36

[90] R. Brüning, K. Samwer, Physical Review B, 1992, 46, 18, 319-322

[91] H.E. Kissinger, ACS Publications, 1957, 29, 11, 1702-1706

[92] A. Concustell, persönliche Mitteilung, April 2009, Department of Materials Science \& Metallurgy, University of Cambridge, Pembroke Street, CB2 3QZ Cambridge, United Kingdom

[93] D. Rodney, C.A. Schuh, Physical Review B, 2009, 80, 184203

[94] E.I. Corwin, H.M. Jaeger, S.R. Nagel, Nature, 2005, 435, 1075-1078

[95] E.I. Corwin, E.T. Hoke, H.M. Jaeger, S.R. Nagel, Physical Review E, 2008, 77, 061308

[96] M. Cloitre, R. Borrega, F. Monti, L. Leibler, Physical Review Letters, 2003, 90, 6, 068303

[97] J. Mattsson, H.M. Wyss, A. Fernandez-Nieves, K. Miyazaki, Z. Hy, D.R. Reichman, D.A. Weitz, Nature, 2009, 462, 83

[98] J.C. Dyre, Review of Modern Physics, 2006, 78, 953-972

[99] S.V. Khonik, V.V Sviridov, O.P. Bobrav, M. Yu Yazivtsky, V.A. Khonik, J. Phys.: Condensed Matter, 2008, 20, 165204

[100] A.V. Granato, Metallurgical and Materials Transactions A, 1998, 29, 7, $1837-1843$

[101] M. Vogel, B. Doliwa, A. Heuer, S.C. Glotzer, Journal of Chemical Physica, 2004, 120, 9, 4404-4414 
[102] C.A Schuh, T.C. Hufnagel, U. Ramamurty, Acta Materialia, 2007, 55, $4067-4109$

[103] J. Lu, G. Ravichandran, W.L. Johnson, Acta Materialia, 2003, 51, 3429-3443

[104] R. David, C. Schuh, Physical Review Letters, 2009, 10223, 235503

[105] P. Guan, M. Chen, T. Egami, Physical Review Letters, 2010, 104, 20, 205701

[106] N. Mattern, J. Bednarcik, S. Pauly, G. Wang, J. Das, J. Eckert, Acta Materialia, 2009, 57, 4133-4139

[107] F.H. Dalla Torre, D. Klaumünzer, R. Maaß, J.F. Löffler, Acta Materialia, 2010, 58, 3742-3750

[108] S.G. Mayr, Physical Review B, 2009, 79, 060201

[109] Y.Q. Cheng, H.W. Sheny, E. Ma, Physical Review B, 2008, 78, 014207

[110] R. Birringer, persönliche Mitteilung, September 2009, University of Saarbrücken, FB 12.1, Bau 2, D-6600 Saarbrücken, FRG

[111] P. Rösner, K. Samwer, P. Lunkenheimer, Europhys. Lett., 2004, 68, 226

[112] R. Rambousky, M. Moske, K. Samwer, Z. Phys. B: Condens. Matter, 1996, 99, 387

[113] M. Weiss, M. Moske, K. Samwer, Phys. Rev. B, 1998, 58, 9062

[114] H.S. Chen, M. Goldstein, J. Appl. Phys., 1972, 43, 1642

[115] S.S. Tsao, F. Spaepen, Acta Metall., 1985, 33, 881

[116] M.L. Cerrada, J.L. de la Fuente, E.L. Madruga, M. Fernández-García, Polymer, 2002, 43, 2803

[117] C.O. M'Bareck, Q.T. Nguyen, M. Metayer, J.M. Saiter, M.R. Garda, Polymer, 2004, 45, 4181 
[118] H. Hart, L.E. Craine, D.J. Hart, C.M. Hadad, Organische Chemie. 3. Auflage, 2007, WILEY-VCH Verlag GmbH \& Co. KGaA, Weinheim

[119] F.A. Carey, R.J. Sundberg, Organische Chemie, 1996, VCH, Weinheim, New York

[120] L. Gargallo, M. Russo, Colloid \&5 Polymer Science, 1977, 255, 11, 11421142

[121] P. Eyerer, J. Woidasky, Einführung in Polymer Engineering, 2008, Springer

[122] Y. Zhang, M. Pan, M.C. Liu, J. Huang, Journal of Polymer Science Part A: Polymer Chemistry, 2008, 46, 8, 2624-2631

[123] M. Schwabe, Characterization of poly (tert-butyl acrylate) and ABAblock-copolymers of tert-butyl acrylate and acrylic acid synthesized via RAFT polymerization, 2009, report of project week within GRK 782 


\section{Lebenslauf}

\section{Persönliche Angaben:}

Name

Geboren am

Geburtsort

Familienstand

Staatsangehörigkeit

\section{Schulausbildung:}

$1988-1992$

$1992-2001$

22. Juni 2001

\section{Grundwehrdienst:}

November 2001 - Juli 2002

\section{Hochschulausbildung:}

Oktober 2002 - November 2007

19. Oktober 2004

09. November 2007

Seit Dezember 2007
Moritz Schwabe

12. 12.1981

Preetz (Schleswig-Holstein)

Verheiratet

Deutsch

Grundschule Altenholz-Stift

Gymnasium Altenholz

Erwerb der Allgemeinen Hochschulreife
Chemiestudium, Universität Göttingen Vordiplom in Chemie

Abschluss Diplom, Note: sehr gut

Diplomthema: Quantitativer Arsennachweis mittels laser-induzierter Plasma-Spektroskopie

(in Zusammenarbeit mit dem

Laser Laboratorium Göttingen GmbH)

Promotion im 1. Physikalischen Institut

der Universität Göttingen im Rahmen eines

Stipendiums des Graduiertenkollegs 782

Promotionsthema: Relaxationen in komplexen Fluiden 


\section{Publikationen}

2010

M. Schwabe, R. Rotzoll, S. Küchemann, K. Nadimpalli, P. Vana and K. Samwer:

Relaxation Processes of Poly (tert-butyl acrylate) Chemically Confined via Hydrogen Bonds

Macromolecular Cemistry and Physics, 2010, 211, 15, 1673 - 1677

M. Schwabe, S. Küchemann, H. Wagner, D. Bedorf and K. Samwer:

Activation volume of microscopic processes in amorphous $P d_{77.5} C u_{6.0} S i_{16.5}$ due to stress and temperature

Journal of Non-Crystalline Solids, 2010, doi:10.1016/j.jnoncrysol.2010.08.019 



\section{Danksagung}

Für die Möglichkeit, als Quereinsteiger aus der Chemie, in der Physik zu promovieren, möchte ich mich ganz herzlich bei Herrn Professor Samwer bedanken. Die freien Räume zur Entstehung meiner eigenen Ideen und die Chance, fächerübergreifend zu arbeiten, waren maßgeblich mitentscheidend für das erfolgreiche Voranschreiten dieser Arbeit. Ebenfalls vielen Dank für die langen Diskussionen und Ihre verständnisvolle Führung unserer Arbeitsgruppe. Außerdem geht mein Dank an Herrn Professor Krebs für die nette Co-Betreuung und die offenen Ohren beim Vorstellen meiner Ergebnisse und deren Diskussion.

Ebenfalls möchte ich mich bei Herrn Professor Vana und Robert Rotzoll für die sehr gute Zusammenarbeit hinsichtlich des Projektes der Block-Copolymere bedanken. An dieser Stelle muss auch an das Graduiertenkolleg 782 gedacht werden, welches die Finanzierung dieser Arbeit übernahm. Hierbei geht vor allem mein Dank an Herrn Professor Suhm und allen Mitgliedern des GRK für die netten Stunden in Paris.

Der gesamten Glasgruppe (Dennis, Stefan, Hannes und Antje) danke ich für die lange Unterstützung bei dieser Arbeit, allen Hilfen und den vielen Stunden am Teilchenbeschleuniger. Ihr ward ein echter Rückhalt in der Abteilung. Auch Uta und Katrin vielen Dank für die Hilfen an der DMA und der DSC und der restlichen 1. Physik für die sehr verständnisvolle und nette Aufnahme als Fachfremder aus der Chemie. An Friedrich Romstedt geht ein besonderes Dankeschön für die Berechnungen und die Hilfe beim Schreiben des Programmes für meine Auswertung der Creep/Recoverykurven.

Außerdem danke ich meinen Eltern für Ihre Hilfe, meiner Schwester für fächerübergreifende Diskussionen und allen meinen Freunden für Ihre Unterstützungen. 
Zum Schluss geht mein besonderer Dank an den wichtigsten Menschen. Vielen Dank, dass du mich mit Deiner ganzen Liebe unterstützt, alle Klagen von mir angehört und mich immer wieder aufgebaut hast. Dass ich dich zur Ehefrau an meiner Seite habe, ist ein ganz großes und besonderes Geschenk, vielen Dank Claudia, dass es dich gibt. Du hast einen Ehrenplatz in dieser Arbeit verdient.

Beschliessen möchte ich diese Arbeit mit dem Zitat des Anfanges:

Die Wahrheit ist eben kein Kristall, den man in die Tasche stecken kann, sondern eine unendliche Flüssigkeit, in die man hineinfällt. (Robert Musil)

Ich habe das Schwimmen gelernt. 